\title{
Representações de Gelfand-Tsetlin de álgebras de Vertex
}

\author{
Oscar Armando Hernández Morales
}

\author{
TESE APRESENTADA \\ $\mathrm{AO}$ \\ Instituto de Matemática e Estatística \\ DA \\ UNIVERSIDADE DE SÃo PAUlo \\ PARA \\ OBTENÇÃO DO TÍTULO \\ $\mathrm{DE}$ \\ Doutor EM CIÊNCIAS
}

\author{
Programa: Matemática \\ Orientador: Prof. Dr. Vyacheslav Futorny \\ Coorientador: Prof. Dr. Luis Enrique Ramirez
}

O autor recebeu auxílio financeiro da CAPES durante o desenvolvimento deste trabalho 


\section{Representações de Gelfand-Tsetlin de álgebras de vértice}

Esta versão da tese contém as correções e alterações sugeridas pela Comissão Julgadora durante a defesa da versão original do trabalho, realizada em 12/03/2021. Uma cópia da versão original está disponível no Instituto de Matemática e Estatística da Universidade de São Paulo.

Comissão Julgadora:

- Prof. Dr. Vyacheslav Futorny (orientador) - IME-USP

- Prof. Dr. Cristián Andrés Ortiz González - IME-USP

- Prof. Dr. Jethro William van Ekeren - UFF

- Prof. Dr. Viktor Bekkert - UFMG

- Prof. Dr. Lucas Henrique Calixto - UFMG 


\section{Agradecimentos}

Em primeiro lugar, agradeço ao Prof. Dr. Vyacheslav Futorny, pela dedicação e paciência na orientação da tese, e pelos ensinamentos ao longo desses anos de trabalho juntos.

Minha gratidão também ao Prof. Dr. Luis Enrique Ramirez, pelas significativas considerações para o encaminhamento da pesquisa e pela presença constante nas dificuldades.

Agradeço à Capes pela bolsa de estudos concedida para a realização da pesquisa.

Aos amigos do IME-USP, que de várias maneiras me apoiaram na realização do trabalho, deixo registrado meu agradecimento. Pela generosa ajuda nos momentos de dúvida e dificuldades durante a elaboração desse trabalho, um agradecimento especial aos amigos André Silva de Oliveira, Marcela Guerrini Alves e Felipe Rocha Félix.

Um agradecimento especial dedico a minha família que, mesmo distante, sempre buscou me ajudar da melhor maneira possível para que tudo desse certo comigo neste país.

Ao Henrique e à Zósia, pelos momentos agradáveis na companhia de boa comida, meu muito obrigado. Agradeço também ao Orlando, pelos churrascos nas festas de Natal, e à Izabel, pela comida farta.

Agradeço à Flávia pelo convívio nos momentos de alegria e pela compreensão e carinho nos momentos difíceis. Ainda, agradeço a ela por ter me "abrasileirado". 


\section{Resumo}

\section{HERNÁNDEZ MORALES, O. A. Representações de Gelfand-Tsetlin de álgebras de Ver-}

tex. 2021. 159 f. Tese (Doutorado) - Instituto de Matemática e Estatística, Universidade de São Paulo, São Paulo, 2021.

Neste trabalho realizamos todos os $\mathfrak{s l}_{n+1}$-módulos de peso máximo simples de relações, isto engloba as famílias construídas em [Maz03] e [FRZ19]. Ademais, para uma subálgebra parabólica de $\mathfrak{s l}_{n+1}$ com subálgebra de Levi $\mathfrak{s l}_{2}+\mathfrak{h}$ construímos uma extensa família de $\mathfrak{s l}_{n+1}$-módulos de relações como imagens do funtor de localização torcida de $\mathfrak{s l}_{n+1}$-módulos de peso máximo simples de relações. Como aplicações, temos a construção explícita em termos de tabelas de Gelfand-Tsetlin de todos os $\mathfrak{s l}_{n+1}$-módulos de peso máximo simples admissíveis, os quais foram anteriormente descritos por Arakawa [Ara16]. Além disso, obtemos duas novas famílias de representações irredutíveis de energia positiva da álgebra de vertex simples afim $V_{k}\left(\mathfrak{s l}_{n+1}\right)$ na órbita nilpotente minimal e órbita nilpotente principal de $\mathfrak{s l}_{n+1}$, respectivamente. Essas representações são quocientes de módulos induzidos para a álgebra de Kac-Moody $\widehat{\mathfrak{s l}}_{n+1}$ e incluem, em particular, todos os módulos simples admissíveis induzidos de $\mathfrak{s l}_{2}$. Assim, completamos alguns dos resultados apresentados em [AFR17].

Palavras-chave: Álgebra de Kac-Moody afim, álgebra de vertex afim, álgebra de Zhu, funtor de localização torcida, módulos Gelfand-Tsetlin, realização por tabelas. 


\section{Abstract}

HERNÁNDEZ MORALES, O. A. Gelfand-Tsetlin representations of Vertex algebras. 2021. 159 f. Tese (Doutorado) - Instituto de Matemática e Estatística, Universidade de São Paulo, São Paulo, 2021.

In this work we explicitly construct all simple highest weight relation $\mathfrak{s l}_{n+1}$-modules. This includes the families constructed in [Maz03] and [FRZ19]. In addition, for a parabolic subalgebra of $\mathfrak{s l}_{n+1}$ with a Levi subalgebra $\mathfrak{s l}_{2}+\mathfrak{h}$ we construct a large family of relation $\mathfrak{s l}_{n+1}$-modules as images under the twisted localization functor of simple highest weight relation $\mathfrak{s l}_{n+1}$-modules. As an application, we have an explicit construction in terms of Gelfand-Tsetlin tableaux, of all admissible simple highest weight $\mathfrak{s l}_{n+1}$-modules, which were previously described by Arakawa in [Ara16]. Furthermore, we obtain two new families of simple positive energy representations of simple affine vertex algebra $V_{k}\left(\mathfrak{s l}_{n+1}\right)$ in the minimal nilpotent orbit and principal nilpotent orbit of $\mathfrak{s l}_{n+1}$, respectively. These representations are quotients of induced modules over the affine Kac-Moody

algebra $\widehat{\mathfrak{s l}}_{n+1}$, they include, in particular, all admissible simple modules induced from $\mathfrak{s l}_{2}$. Thus, we have completed some of the results presented in [AFR17].

Keywords: Affine Kac-Moody algebra, affine vertex algebra, Zhu algebra, twisted localization functors, Gelfand-Tsetlin modules, tableaux realization. 


\section{Sumário}

Introdução $\quad$ xi

1 Módulos de peso para álgebras de Lie simples $\quad 1$

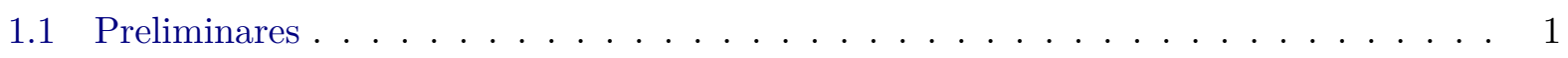

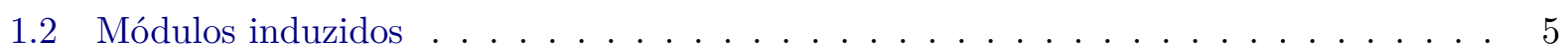

1.3 Funtor de torção e $\alpha$-Gelfand-Tsetlin módulos . . . . . . . . . . . . . . . . . 6

1.4 Teoria de Mathieu . . . . . . . . . . . . . . . . . . . . . 10

2 Álgebras de vertex $\quad 19$

2.1 Álgebras de Kac-Moody afim _ . . . . . . . . . . . . . . . . . . . . . . 19

2.2 Álgebras de vertex e suas representações . . . . . . . . . . . . . . . . . . . . . . . . . . . . . . . . . . . . . . . . .

2.3 Álgebra de Zhu . . . . . . . . . . . . . . . . . . . . . 27

2.4 Representações de energia positiva de $\mathcal{V}_{\kappa}(\mathfrak{g}) \ldots \ldots \ldots \ldots \ldots$

3 Módulos de Gelfand-Tsetlin $\quad 41$

3.1 Convenções . . . . . . . . . . . . . . . . . . . . . . . . . . . . . 42

3.2 Módulos de Gelfand-Tsetlin para $\mathfrak{g l}_{n} \ldots \ldots \ldots$. . . . . . . . . . . 42

3.3 Módulos de $\Gamma_{s t}$-relações para $\mathfrak{g l}_{n} \ldots \ldots \ldots \ldots \ldots \ldots \ldots$

3.4 Módulos de Gelfand-Tsetlin para $\mathfrak{s l}_{n+1} \ldots \ldots \ldots \ldots \ldots \ldots$. . . . . . . . . 54

3.5 Localização torcida de módulos de $\Gamma_{s t}$-relações $\ldots \ldots \ldots$. . . . . . . . . . . . 64

3.6 Família de módulos induzidos de $\Gamma_{s t}$-relações $\ldots \ldots \ldots$. . . . . . . . . . 72

3.7 Localização de módulos de peso máximo com relação a $\alpha_{1 m} \ldots$. . . . . . . . . . . . 74

3.8 Realização de famílias de módulos de $\Gamma_{s t}$-relações para $\mathfrak{s l}_{4} \ldots \ldots \ldots$. . . . . . . 84

4 Módulos admissíveis para $\mathfrak{s l}_{n+1} \quad 99$

4.1 Módulos simples na órbita nilpotente minimal para $\mathfrak{s l}_{n+1} \ldots \ldots \ldots$. . . . . . . 99

$4.2 \mathfrak{s l}_{3}$-módulos de $\Gamma_{\mathcal{F}}$-relações na órbita minimal $\ldots \ldots \ldots$. . . . . . . . . . . . . . . .

4.3 Módulos simples na órbita nilpotente principal para $\mathfrak{s l}_{n+1} \ldots \ldots$. . . . . . . . 113

$\begin{array}{lr}\text { A Grupo de Weyl } & 119\end{array}$

$\begin{array}{ll}\text { B Códigos dos reticulados de peso } & 127\end{array}$

Referências Bibliográficas 133 


\section{Introdução}

A teoria de representações de álgebras de vertex subjacente a uma teoria de campos conformais é tradicionalmente assumida como tendo um módulo de peso máximo como ponto de partida, especialmente quando a teoria em questão é racional. Em particular, os módulos de peso máximo relaxados para álgebras de Kac-Moody afim atraem um interesse considerável devido à sua conexão com a teoria de representações de álgebras de vertex conformais e teoria de campos conformais, de acordo com [FST98, AM95, Ada16] e suas referências. Esses módulos foram originalmente nomeados em [FST98] e definidos para a álgebra de vertex simples afim (nível admissível) $V_{k}\left(\mathfrak{s l}_{2}\right)$.

A ideia por trás da denominação "relaxado" vem do relaxamento da definição de um vetor de peso máximo, para que ele não precise mais ser aniquilado pelos elementos de álgebra associados a raízes positivas. Um módulo relaxado de peso máximo pode ser definido como um módulo que é gerado por um vetor relaxado de peso máximo. Essa ideia pode ser aplicada a classes gerais de álgebras de vertex e, portanto, os módulos de peso máximo relaxados são ingredientes potencialmente importantes de uma ampla variedade de teorias de campo conformais, especialmente na construção de exemplos de álgebras de vertex não racionais.

No entanto, de acordo com os objetivos deste trabalho, a razão mais importante para estudar módulos relaxados de peso máximo é o fato de formarem a maior classe de módulos de peso aos quais os métodos de classificação de Zhu (ver [Zhu96]) podem ser aplicados.

A álgebra de Zhu desempenha um papel muito importante na teoria de álgebras de vertex. Uma vez que há uma correspondência um a um entre as classes de isomorfismo dos módulos admissíveis irredutíveis da álgebra de vertex e as classes de isomorfismo dos módulos irredutíveis de sua álgebra de Zhu [FZ92, Zhu96]. A álgebra de Zhu foi introduzida pela primeira vez por Y.C. Zhu em seu trabalho de tese (veja [Zhu96]). Em 1998, Dong, Li e Mason [DLM98] generalizaram a álgebra de Zhu a uma série de álgebras associativas que chamamos álgebras de Zhu de nível superior. Dong, Li e Mason provaram resultados semelhantes sobre a correspondência entre representações de álgebras de Zhu de nível superior e as de álgebras de operadores de vertex. Em [FZ92], Frenkel e Zhu mostraram que a álgebra de Zhu é isomorfa a um subquociente da álgebra envolvente universal de uma álgebra de Lie de dimensão finita e em [He17], He generalizou essa afirmação e provou que as álgebras de Zhu de nível superior são isomorfas a subquocientes desta álgebra universal.

A teoria de representações de álgebras de vertex e de suas álgebras de Zhu são relacionadas (ver [Zhu96]) pelo funtor Zhu[-]. Usando o funtor de Zhu, o estudo de representações de energia positiva de álgebras de vertex afim simples se reduz às representações de álgebra de Lie subjacente de dimensão finita. O uso de tal funtor permite construir novas famílias de representações simples de álgebras de vertex admissíveis ${ }^{1}$.

\footnotetext{
${ }^{1} \mathrm{~A}$ álgebra de vertex afim $V_{k}(\mathfrak{g})$ é chamada admissível se é isomorfa a uma representação admissível como um $\widehat{\mathfrak{g}}$-módulo, ver [KW89].
} 
Para a álgebra de vertex afim $V_{k}(\mathfrak{g})$, este funtor mapeia a categoria de $V_{k}(\mathfrak{g})$-módulos de peso máximo relaxados para a categoria de $Z_{k}$-módulos de peso, em que $Z_{k}:=\mathrm{Zhu}\left(V_{k}(\mathfrak{g})\right)$ é a álgebra de Zhu de nível $k$ da álgebra de vertex afim $V_{k}(\mathfrak{g})$, a qual por sua vez (segundo [FZ92]) é isomorfa a $\frac{U(\mathfrak{g})}{I_{k}}$, para algum ideal bilateral de $\operatorname{Zhu}\left(V^{k}(\mathfrak{g})\right) \simeq U(\mathfrak{g})$. Ainda segundo [FZ92], temos que qualquer $V_{k}(\mathfrak{g})$-módulo é naturalmente um módulo sobre a álgebra de Kac-Moody afim não torcida $\widehat{\mathfrak{g}}=\mathfrak{g}\left[t, t^{-1}\right] \oplus \mathbb{C} K$ (na qual o elemento central $K$ age como multiplicação por $k$ ), assim de acordo com [KR19a] este funtor tem a forma $Z h u[M]=M^{t g[t]}$ (os elementos de $M$ que são aniquilados por $\operatorname{tg}[t])$. Da mesma forma, existe um funtor Ind $[-]$ da categoria de $Z_{k}$-módulos de peso para a categoria de $V_{k}(\mathfrak{g})$-módulos de peso máximo relaxados, obtidos por "indução" e quociente pelo submódulo maximal cuja interseção com o módulo original é zero. Neste contexto, o significado de "induzir" pode ser encontrado na seção 2.2 em [Zhu96] e na seção 3.2 em [FZ92]. Usando esses dois funtores, Zhu provou o seguinte Teorema.

\section{Teorema de Zhu}

a) Para qualquer representação de energia positiva $M$ de uma álgebra de vertex $\mathcal{V}$, a representação $\bar{a} \mapsto o(a)$ de $\operatorname{Zhu}(\mathcal{V})$ em $M_{\text {top }}$ está bem definida.

b) A correspondência $M \mapsto M_{t o p}$ fornece uma bijeção entre o conjunto de classes de isomorfismo de representações de energia positiva irredutíveis de $\mathcal{V}$ e a de $Z h u(\mathcal{V})$-módulos simples.

Para classificar os módulos de peso máximo simples relaxados da álgebra de vertex afim $V_{k}(\mathfrak{g})$, basta classificar os módulos de peso simples de $\mathfrak{g}$ que são aniquilados pelo ideal $I_{k}$. Assim, nossa tarefa é classificar todos os módulos de peso simples de $\mathfrak{g}$ aniquilados por $I_{k}$. Essa técnica foi aplicada em [AFR17], obtendo assim novas famílias de módulos simples para a álgebra de vertex afim de $\mathfrak{s l}_{n}$ e foi obtida uma classificação completa de todas as representações irredutíveis de peso máximo relaxado com espaços de peso de dimensão finita no caso $n=3$. Além disso, essa abordagem foi desenvolvida em [KR19a] e [KR19b] usando a classificação de Fernando-Mathieu de módulos de peso simples com multiplicidades de peso finito sobre álgebras de Lie simples de dimensão finita. No entanto, a construção explícita de tais representações é desconhecida ainda e, de fato, só foi concluída para $\mathfrak{g}=\mathfrak{s l}_{2}$ (veja [Maz10]). Veja também no artigo recente [FK20], no qual os funtores de localização e de Wakimoto foram utilizados para construir módulos relaxados de Wakimoto para as álgebras universais e de vertex simples afim. Em [AFR17], a realização por tabelas de módulos de Gelfand-Tsetlin foi usada para descrever famílias admissíveis de tipo $A$. Essa realização fornece uma base e fórmulas explícitas para a ação da álgebra $\mathfrak{s l}_{n+1}$. Baseados nesses resultados mencionados, nosso problema consiste em determinar quais dos $\mathfrak{s l}_{n+1}$-módulos induzidos de $\mathfrak{s l}_{2}$-módulos simples são admissíveis.

Deste modo, o objetivo principal desta tese é caracterizar todos os $\mathfrak{s l}_{n+1}$-módulos simples induzidos de $\mathfrak{s l}_{2}$-módulos simples densos admissíveis, determinando quais deles estão na órbita minimal e quais deles na principal. Mais especificamente, determinamos quais $\mathfrak{s l}_{n+1}$-módulos de peso simples induzidos de $\mathfrak{s l}_{2}$ são admissíveis e pertencem à órbita minimal e à órbita regular, respectivamente. Além disso, provamos que todos estes módulos são de relações, isto é, podem ser realizados por tabelas de Gelfand-Tsetlin em que a ação é dada pelas fórmulas clássicas de Gelfand-Tsetlin (veja Definição 3.4.2). 
Os resultados deste trabalho acima enunciados estão contidos nos artigos [FHR20] e [FHK21], nos quais descrevemos várias novas famílias de representações irredutíveis de peso máximo de energia positiva para a álgebra de vertex afim admissível $V_{k}\left(\mathfrak{s l}_{n+1}\right)$. Essas famílias incluem todos os módulos simples de peso máximo de tipo $A$ descritos em [Ara15b] e os módulos simples induzidos a partir de uma subálgebra $\mathfrak{s l}_{2}$ nas órbitas nilpotente minimal, regular e subregular, os quais têm espaços de peso de dimensão finita. Nossa abordagem é baseada na teoria de módulos de relações de Gelfand-Tsetlin desenvolvida em [FRZ19], em que uma base explícita de tabelas foi construída para diferentes classes de módulos simples de Gelfand-Tsetlin para $\mathfrak{g l}_{n}$. A seguir enunciamos os principais resultados contidos nesta tese, os quais foram demonstrados para a álgebra de Lie simples $\mathfrak{g}:=\mathfrak{s l}_{n+1}$. O primeiro e um dos mais importantes (o qual é uma consequência do Lema 3.4.72) é que todo módulo simples de peso máximo admissível de tipo $A$ é um módulo de relações, uma vez que os pesos destes módulos são dominantes regulares (veja Corolário 3.4.8). Temos, assim, o seguinte resultado:

Teorema 1. Seja $k \in \mathbb{Q}$ um número admissivel para $\mathfrak{g}$. Então todo $\mathfrak{g}$-módulo simples de peso máximo $L(\lambda)$ admissivel de nível $k$ é um módulo de $\Gamma_{s t}$-relações ${ }^{3}$. Mais ainda, todo módulo de peso máximo $L_{\mathfrak{b}}^{\mathfrak{g}}(\lambda)$ é um módulo $\Gamma_{\mathcal{F}}$-relações ${ }^{4}$, para alguma bandeira $\mathcal{F}$, em que $\mathfrak{b}=w \mathfrak{b}_{\text {st }}$.

O Teorema 1, demonstra a importância do estudo dos g-módulos de $\Gamma_{s t}$-relações, o que nos leva ao nosso segundo resultado principal e fornece uma realização por tabelas para os módulos simples de peso máximo (veja Teorema 3.4.9). Desse modo, fornecemos condições necessárias e suficientes para que esses módulos sejam módulos de relações com respeito à subálgebra padrão de Gelfand-Tsetlin, cujo enunciado é o seguinte:

Teorema 2. O módulo de peso máximo simples $L(\lambda)$ é um módulo de $\Gamma_{\text {st }}$-relações se, e somente se, uma das seguintes condiçôes for válida:

a) $\left\langle\lambda+\rho, \alpha^{\vee}\right\rangle \notin \mathbb{Z}_{\leq 0}$, para todo $\alpha \in \Delta^{+} \backslash\left\{\alpha_{k n} \mid k=1, \ldots, n\right\}$.

b) Existem $i, j$ únicos com $1 \leq i \leq j<n$, tais que:

i) $\left\langle\lambda+\rho, \alpha_{k}^{\vee}\right\rangle \in \mathbb{Z}_{>0}$ para cada $k>j$,

ii) $\left\langle\lambda+\rho, \alpha^{\vee}\right\rangle \notin \mathbb{Z}_{\leq 0}$ para todo $\alpha \in \Delta^{+} \backslash\left\{\alpha_{i k} \mid k \geq j\right\}$,

iii) $\left\langle\lambda+\rho, \alpha_{\text {in }}^{\vee}\right\rangle \in \mathbb{Z}_{\leq 0}$.

Em que $\alpha_{r s}:=\alpha_{r}+\ldots+\alpha_{s}$ para $1 \leq r \leq s \leq n$.

Como mencionado anteriormente, o problema equivalente à classificação dos módulos de peso máximo simples relaxados da álgebra de vertex afim universal $V^{k}(\mathfrak{g})$ é a classificação de todos os $\mathfrak{g}$-módulos de peso simples, que ainda está longe de ser resolvido. No entanto, ao restringirmos a categoria dos g-módulos de peso com espaços de peso de dimensão finita, temos a classificação feita por Mathieu em [Mat00], artigo no qual introduziu as chamadas famílias coerentes cujas propriedades reduziram o problema à classificação dos g-módulos de peso máximo que satisfazem

\footnotetext{
${ }^{2}$ Este Lema é uma reformulação do Proposição 5.9 em [FRZ19] para $\mathfrak{g l}_{n}$-módulos.

${ }^{3} \Gamma_{s t}$ denota a subálgebra padrão de Gelfand-Tsetlin (correspondente à cadeia de mergulhos a partir do canto superior esquerdo).

${ }^{4} \Gamma_{\mathcal{F}}=w \Gamma_{\text {st }}$ para algum elemento $w$ do grupo de Weyl $W$.
} 
certas condições (veja Proposição 1.4.13). Assim, em razão do Teorema 1, nosso próximo resultado nos permite determinar quais g-módulos simples de peso máximo limitados são módulos de relações (veja a Proposição 3.4.10).

Teorema 3. $O$ módulo de peso máximo simples $L(\lambda)$ de dimensão infinita é um módulo de $\Gamma_{s t^{-}}$ relações com espaços de peso limitados se, e somente se, uma das seguintes condições for válida:

a) $\left\langle\lambda+\rho, \alpha_{n}^{\vee}\right\rangle \notin \mathbb{Z}_{>0} e\left\langle\lambda+\rho, \alpha_{k}^{\vee}\right\rangle \in \mathbb{Z}_{>0}$ para todo $k<n$;

b) $\left\langle\lambda+\rho, \alpha_{1}^{\vee}\right\rangle \notin \mathbb{Z} e\left\langle\lambda+\rho, \alpha_{k}^{\vee}\right\rangle \in \mathbb{Z}_{>0}$ para todo $k>1$;

c) $\left\langle\lambda+\rho, \alpha_{1}^{\vee}\right\rangle \in \mathbb{Z}_{<0} e\left\langle\lambda+\rho, \alpha_{1 n}^{\vee}\right\rangle \in \mathbb{Z}_{\leq 0} e\left\langle\lambda+\rho, \alpha_{k}^{\vee}\right\rangle \in \mathbb{Z}_{>0}$ para todo $k>1$;

d) Existe um único $i \in\{2, \ldots, n-1\}$ tal que $\left\langle\lambda+\rho, \alpha_{i}^{\vee}\right\rangle \in \mathbb{Z}_{<0},\left\langle\lambda+\rho, \alpha_{i-1, i}^{\vee}\right\rangle \in \mathbb{Z}_{>0},\left\langle\lambda+\rho, \alpha_{i n}^{\vee}\right\rangle \in$ $\mathbb{Z}_{\leq 0}$ e $\left\langle\lambda+\rho, \alpha_{k}^{\vee}\right\rangle \in \mathbb{Z}_{>0}$ para todo $k \neq i$

e) Existe um único $i \neq n$ tal que $\left\langle\lambda+\rho, \alpha_{i}^{\vee}\right\rangle \notin \mathbb{Z},\left\langle\lambda+\rho, \alpha_{i+1}^{\vee}\right\rangle \notin \mathbb{Z},\left\langle\lambda+\rho, \alpha_{i, i+1}^{\vee}\right\rangle \in \mathbb{Z}_{>0}$ e $\left\langle\lambda+\rho, \alpha_{k}^{\vee}\right\rangle \in \mathbb{Z}_{>0}$ para todos $k \neq i, i+1$.

O nosso próximo resultado é uma consequência direta do Teorema 1, porém devido à sua importância teórica o destacamos aqui pois trata-se de um critério que nos permite classificar todos os módulos de Verma simples que são de relações (veja o Corolário 3.4.12). Vale notar que este mesmo problema foi resolvido parcialmente em [Maz98], mais especificamente, a Proposição 1 em [Maz98] (ver também [FRZ19]) estabelece a condição necessária do (item a) no seguinte teorema. Além do mais, sabemos que existem (pelo menos para $\mathfrak{s l}_{3}$ ) módulos de Verma generalizados que são admissíveis (veja o Exemplo 4.1.1). Portanto, um primeiro passo no estudo dos módulos de peso máximo relaxados é saber quais módulos simples de Verma generalizados com pesos regulares são de relações. Isto é possível devido à caraterização de Jantzen apresentada em [Jan77] (veja Teorema 1.3.9) e o Teorema 1 (veja o Corolário 3.4.14).

Teorema 4. Seja $\lambda \in \mathfrak{h}^{*}$.

a) O módulo de Verma $M(\lambda)$ é um módulo simples de $\Gamma_{\text {st }}$-relações, se, e somente se,,$\left\langle\lambda+\rho, \alpha^{\vee}\right\rangle \notin$ $\mathbb{Z}$, para qualquer $\alpha \in \Delta^{+} \backslash\left\{\alpha_{k, n} \mid k=1, \ldots, n\right\}$ e $\left\langle\lambda+\rho, \alpha_{k, n}^{\vee}\right\rangle \notin \mathbb{Z}_{>0}$ para $k=1, \ldots, n$.

b) Suponhamos que $\lambda \in \mathfrak{h}^{*}$ regular. O $\mathfrak{g}$-módulo de Verma generalizado $M_{\mathfrak{p}}^{\mathfrak{g}}(\lambda)$ é um $\Gamma_{s t}$-módulo de relações simples, se, e somente se, , $\left\langle\lambda+\rho, \alpha^{\vee}\right\rangle \in \mathbb{Z}_{>0}$ para todo $\alpha \in \Delta_{\mathfrak{l}}^{+},\left\langle\lambda+\rho, \alpha^{\vee}\right\rangle \notin \mathbb{Z}$ para cada $\alpha \in \Delta_{\mathfrak{u}}^{+} \backslash\left\{\alpha_{k, n} \mid k=1, \ldots, n\right\}, e\left\langle\lambda+\rho, \alpha_{k, n}^{\vee}\right\rangle \notin \mathbb{Z}_{\geq 0}$ para qualquer $\alpha_{k, n} \in \Delta_{\mathfrak{u}}^{+}$tal que $k=1, \ldots, n$.

Com relação à teoria de representações de álgebras de vertex, a partir da caracterização dos pesos admissíveis na órbita nilpotente minimal (2.24) dada por Arakawa (veja Proposição $2.10 \mathrm{em}$ [AFR17]) e aplicando o Teorema 3, podemos descrever explicitamente todos os g-módulos de peso máximo simples admissíveis nesta órbita (Teorema 4.1.3). Por outro lado, baseados na caracterização dos pesos na órbita nilpotente principal dada por Kac em [FKW92] (veja Proposição 2.4.15) e o Teorema 4.3.1, apresentamos uma realização explícita de todos os módulos simples de peso máximo na órbita regular (veja a Proposição 4.3.2), os quais são módulos de Verma. Temos assim o seguinte 
resultado:

Teorema 5. Todo g-módulo de peso máximo simples admissivel na órbita minimal é limitado e aqueles que estão na órbita principal são módulos de Verma.

Neste trabalho, com o objetivo de determinar novos módulos simples com o mesmo anulador aplicamos duas ferramentas técnicas principais: o funtor de localização torcida definido por Mathieu em [Mat00] e o funtor de torção na categoria de g-módulos de peso definido em [FK19b]. Se for uma raiz simples, o funtor de torção $T_{\alpha}$ está relacionado ao funtor de torção de Arkhipov [Ark04] na categoria $\mathcal{O}$. Seja $\mathfrak{p}$ uma subálgebra parabólica de $\mathfrak{g}$. Aplicando o funtor de torção $T_{\alpha}$ ao $\mathfrak{g}$-módulo de Verma generalizado $M_{\mathfrak{p}}^{\mathfrak{g}}(\lambda)$ induzido a partir do p-módulo de peso máximo $\lambda$ simples de dimensão finita, obtém-se o $\alpha$-Gelfand-Tsetlin $\mathfrak{g}$-módulo $W_{\mathfrak{p}}^{\mathfrak{g}}(\lambda)$ com $\Gamma_{\alpha}$-multiplicidades finitas, em que $\Gamma_{\alpha}$ é a subálgebra comutativa de $U(\mathfrak{g})$ gerada pela subálgebra de Cartan $\mathfrak{h}$ e pelo centro de $U\left(\mathfrak{s}_{\alpha}\right)$, em que $\mathfrak{s}_{\alpha}$ é a subálgebra de Lie de $\mathfrak{g}$ dada pela $\mathfrak{s l}_{2}$-tripla associada à raiz $\alpha$. Os $\mathfrak{g}$-módulos $W_{\mathfrak{b}}^{\mathfrak{g}}(\lambda)$ são módulos cíclicos de peso em relação à subálgebra de Cartan $\mathfrak{h}$ com subespaços de peso de dimensão infinita se $\alpha$ não for uma raiz simples. Por outro lado, se $\alpha$ é uma raiz simples, esses módulos são g-módulos de Verma torcidos pela conjugação da ação de $\mathfrak{g}$. Para mais detalhes consulte [AL03, FK19a] ou os capítulos 10 e 12 em [Hum08].

A seguir realizamos uma família de módulos de Verma generalizados simples (todos estes módulos com espaços de peso de dimensão limitada) como módulos de relações, em seguida aplicamos a esta família de módulos o funtor de torção $T_{\alpha} \operatorname{com} \alpha$ não necessariamente simples. Além disso, aplicamos o funtor de localização (definido sobre um conjunto multiplicativo com um número finito de geradores comutativos associados a um certo conjunto de raízes, não todas simples) aos módulos de peso máximo simples limitados de relações (veja a Proposição 3.7.6).

Teorema 6. Seja $\lambda \in \mathfrak{h}^{*}$ um peso satisfazendo as condições do Teorema 3 e $F:=\left\{f_{\alpha} \mid \alpha \in \Omega\right\}$ em que $\Omega=\left\{\alpha_{1 i} \mid i=1, \ldots, m\right\}$, para algum $m \in\{1, \ldots, n\}$.

a) Se $\lambda$ satisfaz as condições b), c) ou e) com $i=1$, então $D_{F} L(\lambda)$ é um módulo limitado de $\Gamma_{\text {st }}$-relações.

b) Se $f_{\alpha}$ age injetivamente em $L(\lambda)$ para algum $\alpha \in F$, então $T_{\alpha}(L(\lambda))$ é isomorfo a um módulo de peso máximo simples limitado de $\Gamma_{\text {st }}$-relações para alguma subálgebra de Borel torcida pelo grupo de Weyl. Em particular, se $\lambda$ satisfaz a condição b) do Teorema 3 e $\mathfrak{p}=\mathfrak{p}_{\Sigma}$ é a subálgebra parabólica de $\mathfrak{g}$ correspondente ao subconjunto de raízes simples $\Sigma=\left\{\alpha_{2}, \ldots, \alpha_{n}\right\}$, então o $\mathfrak{g}$-módulo de Verma generalizado $M_{\mathfrak{p}}^{\mathfrak{g}}(\lambda)$ e o $\alpha$-Gelfand-Tsetlin $\mathfrak{g}$-módulo $W_{\mathfrak{p}}^{\mathfrak{g}}(\lambda)$ são módulos simples limitados de $\Gamma_{\text {st }}$-relações.

Além disso, no Teorema 3.6.5, obtemos uma construção explícita de uma família de g-módulos simples que são induzidos parabolicamente a partir de $\mathfrak{s l}_{m}$-módulos densos com espaços de peso de dimensão finita, em que $m \in\{2, \ldots, n\}$.

Teorema 7. Seja $V$ um módulo denso de $\Gamma_{\text {st }}$-relações (construído no Lema 3.6.2) e $\mathfrak{p}=\mathfrak{p}_{\Sigma} a$ subálgebra parabólica correspondente ao subconjunto de raízes simples $\Sigma=\left\{\alpha_{1}, \ldots, \alpha_{m-1}\right\}$ de $\mathfrak{g}$, em que a subálgebra de Levi de $\mathfrak{p}$ é isomorfa com $\mathfrak{s l}_{m}+\mathfrak{h}$, então o $\mathfrak{g}$-módulo induzido simples limitado 
$L_{\mathfrak{p}}^{\mathfrak{g}}(\Sigma, V)$ é de $\Gamma_{\text {st }}$-relações.

Observamos que para $m=2$, provamos que o Teorema anterior continua sendo verdadeiro para módulos não limitados. Para provar esta afirmação, aplicamos o funtor de localização torcida a qualquer módulo de relações tal que a ação de $f_{\alpha_{1}}$ no módulo seja injetiva (veja Teorema 3.5.6). Vale notar que no próximo teorema não precisamos da hipótese sobre a finitude dos espaços de peso.

Teorema 8. Seja $f=f_{\alpha_{1}}, M$ um $\Gamma_{\text {st }}$-módulo de relações com uma ação injetiva de $f$. Então a localização torcida $D_{f}^{x} M$ de $M$ é um módulo de relaçôes de Gelfand-Tsetlin para qualquer $x \in \mathbb{C}$.

Este Teorema nos permite classificar todos os módulos simples de relações de Gelfand-Tsetlin induzidos a partir da $\mathfrak{s l}_{2}$-subálgebra gerada pela tripla $\left(e_{\alpha_{1}}, h_{\alpha_{1}}, f_{\alpha_{1}}\right)$ (veja o Teorema 3.5.9).

Teorema 9. Seja $n>1$. O módulo $L(\gamma, \mu)$ é um módulo de Gelfand-Tsetlin de $\Gamma_{\text {st }}$-relações se, e somente se, $L(\lambda)$ for um $\mathfrak{g}$-módulo de peso máximo de $\Gamma_{s t}$-relações, em que $\left\langle\mu-\lambda, \alpha_{1}^{\vee}\right\rangle=2 x$, $\left\langle\mu-\lambda, \alpha_{2}^{\vee}\right\rangle=-x,\left\langle\mu-\lambda, \alpha_{i}^{\vee}\right\rangle=0$ para cada $i=3, \ldots, n,\left\langle\lambda+\rho, \alpha_{1}^{\vee}\right\rangle \notin \mathbb{Z}_{\geq 0}$ e $\left(2 x-\mu_{1}-1\right)^{2}=\gamma$. Neste caso, $L(\gamma, \mu) \simeq D_{f}^{x}(L(\lambda))$ e x satisfaz a condição $x-\left\langle\mu+\rho, \alpha_{1}^{\vee}\right\rangle \notin \mathbb{Z}$. Além disso,

a) $L(\gamma, \mu)$ é limitado se, e somente se, $L(\lambda)$ for limitado;

b) Se $\lambda$ for dominante, $s_{1} \cdot \lambda$ será dominante e $\gamma \neq m^{2}$ para todo $m \in \mathbb{Z}$. Neste caso, $A n n_{U(\mathfrak{g})} L(\gamma, \mu)=$ $A n n_{U(\mathfrak{g})} L(\lambda)=A n n_{U(\mathfrak{g})} L\left(s_{1} \cdot \lambda\right)$

c) Se $\gamma=m^{2}$ por algum $m \in \mathbb{Z} \backslash\{0\}$, então $L(\lambda)$ é limitado e o peso $w \cdot \lambda$ é integral, para todo $w \in W$. Neste caso, $A n n_{U(\mathfrak{g})} L(\lambda) \subset A n n_{U(\mathfrak{g})} L(\gamma, \mu) \subset A n n_{U(\mathfrak{g})} L(w \cdot \lambda)$, se $w \cdot \lambda$ for dominante.

Aplicando a conjugação pelo grupo de Weyl aos módulos de peso máximo na órbita minimal e localização torcida, junto ao Teorema 9, temos a descrição explícita de todos os g-módulos simples admissíveis induzidos a partir da subálgebra $\mathfrak{s l}_{2}$ na órbita nilpotente minimal. Retomamos estes resultados no nosso próximo Teorema (veja o Teorema 4.1.3 e Teorema 4.1.12).

Teorema 10. Seja $\beta$ uma raiz de $\mathfrak{g}, \mathfrak{b}$ uma subálgebra de Borel de $\mathfrak{g}$ para a qual $\beta$ é uma raiz positiva, $\rho_{\beta}$ a meia soma das raízes positivas (com relação a b ). Seja $L_{\mathfrak{b}}(\lambda)$ um $\mathfrak{g}$-módulo simples e de peso $\mathfrak{b}$-máximo admissível na órbita minimal, de modo que $\left\langle\lambda+\rho_{\beta}, \beta^{\vee}\right\rangle \notin \mathbb{Z}$ e $f=f_{\beta}$. Denotemos por $A_{\mathfrak{b}, \beta}$ o conjunto de todos os $x \in \mathbb{C} \backslash \mathbb{Z}$ para os quais $x+\left\langle\lambda+\rho_{\beta}, \beta^{\vee}\right\rangle \notin \mathbb{Z}$. Então, os módulos localizados torcidos $D_{f_{\beta}}^{x} L_{\mathfrak{b}}(\lambda), x \in A_{\mathfrak{b}, \beta}$ esgotam todos os módulos $\mathfrak{s l}_{2}$-induzidos simples admissiveis na órbita minimal. Mais ainda, existe uma bandeira $\mathcal{F}$ tal que $D_{f_{\beta}}^{x} L_{\mathfrak{b}}(\lambda)$ é um $\mathfrak{g}$-módulo de $\Gamma_{\mathcal{F}}$-relações de Gelfand-Tsetlin.

Como consequência do Teorema anterior, temos que todos os $\mathfrak{s l}_{3}$-módulos admissíveis na órbita minimal são módulos de $\Gamma_{\mathcal{F}}$-relações (veja Teorema 4.2.6). Ressaltamos que na classificação dada previamente no Teorema 5.6 em [AFR17], a realização do $\mathfrak{s l}_{3}$-módulos densos na órbita minimal utiliza tabelas singulares críticas e nós apresentamos uma realização diferente por meio de tabelas genéricas (veja o Lema 4.2.4), isto nos permite afirmar que: 
Teorema 11. Todo $\mathfrak{s l}_{3}$-módulo admissivel simples na órbita minimal é um módulo limitado de $\Gamma_{\mathcal{F}}$-relações.

Nosso próximo Teorema é análogo ao Teorema 10, com a diferença que neste caso realizamos todos os $\mathfrak{g}$-módulos admissíveis simples induzidos a partir da subálgebra $\mathfrak{s l}_{2}$ na órbita principal (veja o Teorema 4.3.2 e o Teorema 4.3.8). É importante notar que o item b) do Teorema a seguir, completamos a classificação de todos os módulos simples admissíveis apresentada no Teorema 6.5 em [AFR17], entretanto no item d) somente descrevemos os módulos admissíveis induzidos de $\mathfrak{s l}_{3}$-módulos densos que podem ser obtidos aplicando localização torcida baseada numa raiz, veja Teorema 6.7 em [AFR17].

\section{Teorema 12.}

Seja $k$ um número admissível para $\mathfrak{g}$ com denominador $q$. Definamos o peso $\Lambda=\lambda-\frac{p}{q}(\mu+\rho)$ para $\mathfrak{g}$, em que $\left\langle\lambda+\rho, \alpha_{i}^{\vee}\right\rangle \in \mathbb{Z}_{>0}$ e $\left\langle\mu+\rho, \alpha_{i}^{\vee}\right\rangle \in \mathbb{Z}_{>0}$, para todo $i=1, \ldots, n,\left\langle\lambda+\rho, \alpha_{1 n}^{\vee}\right\rangle<p$ e $\left\langle\mu+\rho, \alpha_{1 n}^{\vee}\right\rangle<q$. Então,

a) O módulo de peso máximo simples $L(\Lambda)$ é um $\mathfrak{g}$-módulo de $\Gamma_{\text {st-relações de Gelfand-Tsetlin. Além }}$ disso, todos os módulos de peso máximo simples admissíveis na órbita principal são módulos de Verma simples de $\Gamma_{\text {st }}$-relaçôes.

b) Existem números complexos $\left\{u_{1}, u_{2}\right\}$ e $\left\{v_{i}\right\}_{i=1, \ldots, n}$ tais que o módulo induzido $L\left(u_{1}, u_{2}, v_{1}, \ldots, v_{n}\right)$ é um $\mathfrak{g}$-módulo simples de $\Gamma_{\mathcal{F}}$-relações admissível de nível $k$ na órbita principal $\mathbb{O}_{\text {prin }}$. Mais ainda, esses módulos esgotam todos os $\mathfrak{g}$-módulos de peso simples admissiveis na órbita principal induzidos a partir de $\mathfrak{s l}_{2}$.

Nosso último resultado se inspira na construção da família de $\mathfrak{g}$-módulos fortemente genéricos simples na órbita principal, descrita no artigo [AFR17] e no qual os autores afirmam que: "todos os $\mathfrak{g}$-módulos genéricos simples na órbita principal que constituem a família têm espaços de peso de dimensão infinita, com exceção dos módulos de peso máximo e os induzidos de $\mathfrak{s l}_{2}$ ". Neste trabalho provamos a existência de $\mathfrak{s l}_{4}$-módulos simples fortemente genéricos admissíveis na órbita principal com espaços de peso de dimensão finita que são induzidos de $\mathfrak{s l}_{2} \oplus \mathfrak{s l}_{2}$-módulos (veja Lema 4.3.10). Assim, podemos afirmar que:

\section{Teorema 13.}

Os únicos $\mathfrak{s l}_{4}$-módulos admissíveis simples na órbita principal com espaços de peso de dimensão finita (ilimitada) são de $\Gamma_{\mathcal{F}}$-relações e entre eles temos: módulos de Verma, módulos induzidos de $\mathfrak{s l}_{2}$-módulos e módulos induzidos de $\mathfrak{s l}_{2} \oplus \mathfrak{s l}_{2}$-módulos.

Quanto à organização desta tese, o capítulo 1 tem como objetivos introduzir os conceitos básicos abordados neste texto e familiarizar o leitor com as notações que serão utilizadas ao longo do texto. Na seção 1.4, coletamos algumas definições importantes e resultados preliminares relacionados a módulos de peso simples que possuem espaços de peso de dimensão finita, os quais foram previamente classificados por Fernando [Fer90] e Mathieu [Mat00].

No capítulo 2, apresentamos uma síntese de alguns dos resultados básicos da teoria das álgebras de vertex afim e de suas representações, que será utilizada de forma recorrente ao longo da tese. 
A seção 2.1 tem por objetivo introduzir as álgebras de Kac-Moody (seguindo [Kac90]), a partir das quais são definidas as álgebras de vertex estudadas neste trabalho. Na seção 2.2 apresentamos as definições de álgebras de vertex e módulos para estas álgebras seguindo [Kac98, FHL93]. Na seção 2.3 definimos a álgebra de Zhu, módulos admissíveis e o Teorema da correspondência das representações de energia positiva, enunciando os resultados apresentados por Zhu em [Zhu96, FZ92]. Ao final do capítulo (na seção 2.4), apresentaremos brevemente as representações de energia positiva para a álgebra de vertex afim universal seguindo [KW89, KW08]. A seguir apresentamos a definição de $\mathfrak{g}$-módulo admissível estabelecida em [KW89] e o Teorema de Arakawa [Ara15b], o qual estabelece as condições necessárias e suficientes para que um g-módulo simples de peso máximo seja admissível.

No capítulo 3 vamos relembrar a definição da subálgebra de Gelfand-Tsletlin assim como os conceitos relacionados aos módulos de Gelfand-Tsetlin (seção 3.2). Neste mesmo capítulo, na seção 3.3, apresentaremos a teoria sobre módulos de relações para $\mathfrak{g l}_{n}$, que trata das realizações dos módulos de Gelfand-Tsetlin utilizando as tabelas de Gelfand-Tsetlin que satisfazem certos conjuntos de relações chamados admissíveis (ver [FRZ19]). Na seção 3.4, apresentaremos a classificação dos $\mathfrak{s l}_{n+1}$-módulos de peso máximo (limitados e não limitados) de relações como estabelecida em [FHR20], assim como apresentaremos as principais definições e os fatos mais relevantes sobre a construção destes módulos, seguindo diretamente o que foi estabelecido em [FHK21]. Na seção 3.5, aplicamos o funtor de localização torcida em relação a $E_{21}$ e provamos que o módulo ainda é de relações. Em particular, mostramos que todo $\mathfrak{s l}_{n+1}$-módulo simples induzido de $\mathfrak{s l}_{2}$ é um módulo de relações, se, e somente se, o módulo é o localizado torcido de um módulo de peso máximo de relações. Na seção 3.6 construímos uma família de módulos limitados de relações induzidos parabolicamente a partir de um módulo denso de relações (com espaços de peso de dimensão finita). Destacamos que a realização de (quase todos) os módulos densos (com espaços de peso de dimensão finita) foi realizada previamente por Mazorchuck em [Maz03]. O estudo da injetividade e bijetividade dos operadores $E_{m 1}$ para todo o $m \in\{2, \ldots, n\}$ agindo nos módulos limitados de relações está incluído na seção 3.7, o que nos permite realizar $\alpha$-Gelfand-Tsetlin $\mathfrak{s l}_{n+1}$ módulos de relações com espaços de peso de dimensão finita, ainda que $\alpha$ seja a raiz maximal de $\mathfrak{s l}_{n+1}$ (ver [FK19a]). Para finalizar esse capítulo, na seção 3.8 apresentamos diversos exemplos de módulos de relações (genéricos, e singulares não críticos) para a álgebra $\mathfrak{s l}_{4}$, um para cada classe de módulos de peso simples com espaços de peso de dimensão finita.

O capítulo 4 discute os principais resultados desta tese referente à realização de módulos de peso máximo relaxados, a saber: o primeiro resultado (seção 4.1) apresenta a construção explícita por tabelas das seguintes classes de $\mathfrak{s l}_{n+1}$-módulos admissíveis: módulos de peso máximo e induzidos a partir de $\mathfrak{s l}_{2}$-módulos admissíveis densos na órbita nilpotente minimal. O segundo resultado (apresentado na seção 4.3) é uma reformulação do Teorema 5.6 em [AFR17], motivo pelo qual foi preciso dar uma nova construção dos $\mathfrak{s l}_{3}$-módulos densos na órbita minimal. Finalmente, estabelecemos um terceiro resultado: a reformulação dos Teoremas 6.5 e 6.7 em [AFR17], o que nos permite completar a classificação dos $\mathfrak{s l}_{n+1}$-módulos admissíveis induzidos a partir de $\mathfrak{s l}_{2}$-módulos admissíveis densos na órbita nilpotente principal e provar que todos eles são módulos de relações.

Por fim, incluímos dois apêndices: No apêndice A apresentamos o grupo de Weyl para $\mathfrak{s l}_{3}$ e $\mathfrak{s l}_{4}$, além das órbitas de Weyl para cada peso em cada uma destas álgebras e no apêndice B encontram-se os códigos que foram utilizados para construir os reticulados de pesos dos $\mathfrak{s l}_{4}$-módulos simples apresentados ao logo deste trabalho. Ao leitor que não tem familiaridade com esta teoria, 
recomendamos consultar [BB05, Hum80, Dix77, Car05]. 


\section{Módulos de peso para álgebras de Lie simples}

Durante toda a tese, o corpo de base será $\mathbb{C}$, isto é, todos os espaços vetoriais e produtos tensoriais são considerados sobre os números complexos $\mathbb{C}$. Além disso, ao longo desta tese fixaremos $\mathfrak{g}$ como uma álgebra de Lie simples de dimensão finita. Alguns dos resultados mencionados neste trabalho valem em um contexto mais geral; porém, como nosso objetivo é trabalhar com módulos sobre $\mathfrak{s l}_{n+1}$, sobre os quais iremos falar durante a maior parte do trabalho, vamos nos restringir a este caso.

Neste capítulo vamos recordar alguns conceitos preliminares e algumas propriedades que usaremos ao longo deste trabalho. Assumimos que o leitor possui uma noção básica sobre a teoria de álgebras de Lie, noções estas contidas em [Dix77, Hum80, Mar10].

\subsection{Preliminares}

Vamos fixar a notação e a terminologia da tese; deixar explícito o que está sendo assumido, e eventualmente lembrar de algumas definições e resultados.

Seja $\mathfrak{h}$ uma subálgebra de Cartan de $\mathfrak{g}$. Denotamos por $\Delta$ o sistema de raízes de $\mathfrak{g}$ com relação a $\mathfrak{h}$, por $\Delta_{+}$um sistema positivo de raízes em $\Delta$, e por $\Pi \subset \Delta_{+}$a base do conjunto de raízes simples. Para $\alpha \in \Delta_{+}$, seja $h_{\alpha} \in \mathfrak{h}$ a corraiz correspondente. Sejam $e_{\alpha}$ e $f_{\alpha}$ bases dos subespaços raiz $\mathfrak{g}_{\alpha}$ e $\mathfrak{g}_{-\alpha}$, respectivamente, definidos pela relação $\left[e_{\alpha}, f_{\alpha}\right]=h_{\alpha}$. Estabelecemos ainda

$$
Q=\sum_{\alpha \in \Pi} \mathbb{Z} \alpha \quad \text { e } \quad Q_{+}=\sum_{\alpha \in \Pi} \mathbb{Z}_{\geq 0} \alpha,
$$




$$
P=\sum_{\alpha \in \Pi} \mathbb{Z} \omega_{\alpha} \quad \text { e } \quad P_{+}=\sum_{\alpha \in \Pi} \mathbb{Z}_{\geq 0} \omega_{\alpha},
$$

em que $\omega_{\alpha} \in \mathfrak{h}^{*}$, para $\alpha \in \Pi$, é o peso fundamental determinado por $\omega_{\alpha}\left(h_{\gamma}\right)=\delta_{\alpha, \gamma}$, para todo $\gamma \in \Pi$. Chamamos $Q$ de reticulado de raízes, e $P$ de reticulado de pesos. Além disso, definimos o vetor de Weyl $\rho \in \mathfrak{h}^{*}$ por

$$
\rho:=\frac{1}{2} \sum_{\alpha \in \Delta_{+}} \alpha
$$

A subálgebra de Borel canônica $\mathfrak{b}$ de $\mathfrak{g}$ é definida por $\mathfrak{b}=\mathfrak{h} \oplus \mathfrak{n}$, com o nilradical $\mathfrak{n}$ e o nilradical oposto $\overline{\mathfrak{n}}$ definidos do seguinte modo

$$
\mathfrak{n}=\bigoplus_{\alpha \in \Delta_{+}} \mathfrak{g}_{\alpha} \quad \text { e } \quad \overline{\mathfrak{n}}=\bigoplus_{\alpha \in \Delta_{+}} \mathfrak{g}_{-\alpha}
$$

Temos então a correspondente decomposição triangular

$$
\mathfrak{g}=\overline{\mathfrak{n}} \oplus \mathfrak{h} \oplus \mathfrak{n}
$$

da álgebra de Lie $\mathfrak{g}$.

Sejam $\kappa_{\mathfrak{g}}$ a forma de Cartan-Killing em $\mathfrak{g}$, e $(\cdot, \cdot)_{\mathfrak{g}}$ a forma bilinear induzida correspondente em $\mathfrak{g}^{*}$. Sempre que $\alpha \in \mathfrak{h}^{*}$ satisfizer $(\alpha, \alpha)_{\mathfrak{g}} \neq 0$, definimos $s_{\alpha} \in \mathrm{GL}\left(\mathfrak{h}^{*}\right)$ por

$$
s_{\alpha}(\gamma)=\gamma-\frac{2(\alpha, \gamma)_{\mathfrak{g}}}{(\alpha, \alpha)_{\mathfrak{g}}} \alpha
$$

para $\gamma \in \mathfrak{h}^{*}$. O subgrupo $W$ de $\operatorname{GL}\left(\mathfrak{h}^{*}\right)$ dado por

$$
W=\left\langle s_{\alpha} ; \alpha \in \Pi\right\rangle
$$

é chamado de grupo de Weyl de g. Observamos que $W$ é um grupo de Coxeter finito.

Definição 1.1.1 (Definição 4.9 em [Jos98]). Para todo $w \in W$ e $\lambda \in \mathfrak{h}^{*}$, a ação transladada ${ }^{1}$ é definida por $w \cdot \lambda=w(\lambda+\rho)-\rho$.

Observação 1.1.2. Como a ação transladada satisfaz a identidade $w \cdot\left(w^{\prime} \cdot \lambda\right)=w w^{\prime} \cdot \lambda$ para todo $w, w^{\prime} \in W$. Temos que $(w, \lambda) \longrightarrow w \cdot \lambda$ é uma ação do grupo $W$ em $\mathfrak{h}^{*}$.

Denotamos por $\kappa_{0}$ a forma bilinear simétrica, $\mathfrak{g}$-invariante, normalizada em $\mathfrak{g}$ de tal maneira que $(\theta, \theta)=2$, em que $\theta \in \Delta_{+}$é a raiz longa de $\mathfrak{g}$. Ademais, denotamos por $\theta_{s}$ a raiz curta de $\mathfrak{g}$. Então temos $\left(\theta_{s}, \theta_{s}\right)=2 / r^{\vee}$, em que $r^{\vee}$ é o número de laços de $\mathfrak{g}$, ou seja, o número máximo de arestas no diagrama de Dynkin $\mathfrak{g}$. Definimos também

$$
P^{\vee}=\bigoplus_{\alpha \in \Pi} \mathbb{Z} \omega_{\alpha}^{\vee} \quad \text { e } \quad P_{+}^{\vee}=\bigoplus_{\alpha \in \Pi} \mathbb{Z}_{\geq 0} \omega_{\alpha}^{\vee}
$$

\footnotetext{
${ }^{1}$ Ação dot.
} 
em que $\omega_{\alpha}^{\vee} \in \mathfrak{h}^{*}$, para $\alpha \in \Pi$, é o copeso fundamental definido por $\left(\omega_{\alpha}^{\vee}, \gamma\right)=\delta_{\alpha, \gamma}$, para todo $\gamma \in \Pi$. Chamamos $P^{\vee}$ de reticulado de corraízes.

Definição 1.1.3 ([Hum08, Ara17]). Um peso $\lambda \in \mathfrak{h}^{*}$ é chamado:

a) integral dominante se $\left\langle\lambda+\rho, \alpha^{\vee}\right\rangle \in \mathbb{Z}_{>0}$, para todo $\alpha \in \Delta^{+}$;

b) dominante se $\left\langle\lambda+\rho, \alpha^{\vee}\right\rangle \notin \mathbb{Z}_{<0}$ para todo $\alpha \in \Delta^{+}$.

c) antidominante se $\left\langle\lambda+\rho, \alpha^{\vee}\right\rangle \notin \mathbb{Z}_{>0}$, para todo $\alpha \in \Delta^{+}$;

d) regular se $\left\langle\lambda+\rho, \alpha^{\vee}\right\rangle \neq 0$, para todo $\alpha \in \Delta$;

e) dominante regular se $\left\langle\lambda+\rho, \alpha^{\vee}\right\rangle \notin \mathbb{Z}_{\leq 0}$, para todo $\alpha \in \Delta^{+}$.

Exemplo 1.1.4. O peso - $\rho$ não é dominante regular, pois não é regular, mas é dominante e antidominante. Por outro lado, é integral, mas não é integral dominante.

Definição 1.1.5. Seja $M$ um g-módulo.

i) Para $\lambda \in \mathfrak{h}^{*}$, o espaço $M_{\lambda}=\{m \in M \mid h m=\lambda(h) m$, para cada $h \in \mathfrak{h}\}$ é chamado de $\lambda$-espaço de peso de $M$.

ii) $M$ é um módulo de peso se $M=\bigoplus_{\lambda \in \mathfrak{h}^{*}} M_{\lambda}$.

iii) $M$ é chamado limitado se é um módulo de peso, e existe uma constante $C$ tal que $\operatorname{dim} M_{\lambda}<C$, para cada $\lambda \in \mathfrak{h}^{*}$. Diremos que $\max _{\lambda \in \mathfrak{h}^{*}}\left\{\operatorname{dim} M_{\lambda}\right\}$ é o grau de $M$.

\subsubsection{Ações injetoras e finitas}

Definição 1.1.6. Seja $M$ um $\mathfrak{g}$-módulo. Dizemos que um elemento $x$ de $\mathfrak{g}$ age localmente nilpotente (ou, age finitamente) em um vetor $m$ em $M$, se existir $N=N(x, m) \in \mathbb{Z}_{>0}$ tal que $x^{N}(m)=0$. Se esse $N$ não existir, dizemos que $x$ age injetivamente em $m$. Dizemos que $x$ age injetivamente (respectivamente, finitamente) em $M$, se $x$ age injetivamente (respectivamente, finitamente) em todo $m \in M$.

Usaremos frequentemente o seguinte. Seja $x$ um elemento adnilpotente em $\mathfrak{g}$, e seja $M$ um $\mathfrak{g}$-módulo. Então, o conjunto $M^{\langle x\rangle}$ de todos os $m$ nos quais $x$ age finitamente é um submódulo de $M$. Em particular, se $M$ é simples, todo elemento adnilpotente $x$ de $\mathfrak{g}$ age finitamente ou injetivamente em $M$. 


\subsubsection{Notação para $\mathfrak{s l}_{n+1}$}

Escolhemos a decomposição triangular natural de $\mathfrak{s l}_{n+1}$ (a subálgebra de Cartan $\mathfrak{h}$, que consiste nas matrizes diagonais; e subálgebras nilpotentes $\mathfrak{n}^{ \pm}$, que consistem em matrizes triangulares estritamente superiores (respectivamente inferiores)). Denotamos por $\Delta:=\Delta\left(\mathfrak{s l}_{n+1}, \mathfrak{h}\right)$ o sistema de raízes correspondente. Fixamos uma base $\left\{\varepsilon_{1}, \ldots, \varepsilon_{n}\right\}$ de $\mathfrak{h}$ de modo que $\Delta=\left\{\varepsilon_{i}-\varepsilon_{j} \mid i \neq j\right\}$; então $\Pi=\left\{\varepsilon_{i}-\varepsilon_{i+1} \mid i=1, \ldots, n\right\}$ é o conjunto de raízes simples. Para cada raiz $\alpha \in \Delta$, fixamos uma $\mathfrak{s l}_{2}$-tripla $\left(e_{\alpha}, f_{\alpha}, h_{\alpha}\right)$ de forma que $e_{\alpha}$ está no espaço $\alpha$-raiz de $\mathfrak{s l}_{n+1}$ e $f_{\alpha}:=e_{-\alpha}$. Denotamos $\varepsilon_{i}-\varepsilon_{i+1}$ por $\alpha_{i}$.

Lembremos que $Q^{+}:=\sum_{\alpha \in \Pi} \mathbb{Z}_{\geq 0} \alpha$ e introduzimos a ordem parcial usual em $\mathfrak{h}^{*}: \mu \leq \nu$ se $\nu-\mu \in Q^{+}$.

Denotamos a forma bilinear padrão em $\mathfrak{h}^{*}$ por $(\cdot, \cdot)$. Um peso $\lambda_{1} \varepsilon_{1}+\ldots+\lambda_{n} \varepsilon_{n} \in \mathfrak{h}^{*}$ será denotado por $\left(\lambda_{1}, \ldots, \lambda_{n}\right)$.

Denotamos o grupo de Weyl de $\mathfrak{s l}_{n+1}$ por $W$. Uma reflexão em $W$ correspondente a uma raiz $\alpha$ será indicada por $s_{\alpha}$. Seja $s_{i}:=s_{\alpha_{i}}$. Para $1 \leq i, k \leq n-1$, usaremos a seguinte convenção

$$
\prod_{j=i}^{k} s_{j}:= \begin{cases}s_{i} s_{i+1} \ldots s_{k} & \text { se } k \geq i \\ s_{i} s_{i-1} \ldots s_{k} & \text { se } k<i .\end{cases}
$$

O comprimento de um elemento $w$ de $W$, ou seja, a quantidade de reflexões simples em uma expressão reduzida de $w$, será indicado por $l(w)$.

\subsubsection{Série formal}

Para um espaço vetorial $V$, o espaço de polinômios formais de Laurent em $z$ com coeficientes em $V$ é definido por

$$
V\left[\left[z, z^{-1}\right]\right]:=\left\{\sum_{n \in \mathbb{Z}} v_{n} z^{n} \mid v_{n} \in V\right\} .
$$

Ele contém o subespaço das séries de potências formais de Laurent,

$$
V((z)):=\left\{\sum_{n \in \mathbb{Z}} v_{n} z^{n} \mid v_{n} \in V, v_{n}=0 \text { for } n \ll 0\right\} .
$$

O diferencial formal e o resíduo formal de um elemento em $V\left[\left[z, z^{-1}\right]\right]$ são definidos como

$$
\frac{d}{d z}\left(\sum_{n \in \mathbb{Z}} v_{n} z^{n}\right):=\sum_{n \in \mathbb{Z}} n v_{n} z^{n-1}, \quad \operatorname{Res}_{z}\left(\sum_{n \in \mathbb{Z}} v_{n} z^{n}\right):=v_{-1} .
$$




\subsection{Módulos induzidos}

Seguindo as notações de [Fut97] e [DFG09], um subconjunto aditivamente fechado $P \subset \Delta$ é chamado de partição de $\Delta$ se, $P \cap(-P)=\emptyset$ e $P \cup(-P)=\Delta$. Para toda partição $P$ de $\Delta$, definimos a subálgebra de Borel $\mathfrak{b}_{P} \subset \mathfrak{g}$ gerada por $\mathfrak{h}$ e pelos espaços de raízes $a_{\alpha}$, com $\alpha \in P$.

Uma subálgebra parabólica é uma subálgebra que contém uma subálgebra de Borel. Se p é uma subálgebra parabólica de $\mathfrak{g}$ então $\mathfrak{p}=\mathfrak{p}_{0} \oplus \mathfrak{p}_{+}$, onde $\mathfrak{p}_{0}$ é um fator de Levi redutível e $\mathfrak{p}_{+}$é uma subálgebra nilpotente. Subálgebras parabólicas correspondem a uma escolha de base $\Pi$ de um sistema de raízes $\Delta$ e um subconjunto $S \subset \Pi$ (ver [Fut97] e [FK18] para maiores detalhes).

Por $\mathfrak{b}_{s t}=\mathfrak{h} \oplus \mathfrak{n}$, denotamos a subálgebra de Borel canônica de $\mathfrak{g}$, em que $\mathfrak{n}=\bigoplus_{\alpha \in \Delta^{+}} \mathfrak{g}_{\alpha}$. Além dessa subálgebra, consideramos também as subálgebras parabólicas canônicas de g. Para um subconjunto $\Sigma$ de $\Pi$, denotamos por $\Delta_{\Sigma}$ o subsistema de raízes em $\mathfrak{h}^{*}$ gerado por $\Sigma$. Então, a subálgebra parabólica canônica $\mathfrak{p}_{\Sigma}$ de $\mathfrak{g}$, associada a $\Sigma$, é definida como $\mathfrak{p}_{\Sigma}=\mathfrak{l}_{\Sigma} \oplus \mathfrak{u}_{\Sigma}^{+}$, com o nilradical $\mathfrak{u}_{\Sigma}^{+}$e o nilradical oposto $\mathfrak{u}_{\Sigma}^{-}$dados por

$$
\mathfrak{u}_{\Sigma}^{+}=\bigoplus_{\alpha \in \Delta^{+} \backslash \Delta_{\Sigma}} \mathfrak{g}_{\alpha} \quad \text { e } \quad \mathfrak{u}_{\Sigma}^{-}=\bigoplus_{\alpha \in \Delta^{+} \backslash \Delta_{\Sigma}} \mathfrak{g}_{-\alpha}
$$

com a subálgebra de Levi $\mathfrak{l}_{\Sigma}$ definida por

$$
\mathfrak{l}_{\Sigma}=\mathfrak{h} \oplus \bigoplus_{\alpha \in \Delta_{\Sigma}} \mathfrak{g}_{\alpha}
$$

Ademais, temos a decomposição triangular correspondente

$$
\mathfrak{g}=\mathfrak{u}_{\Sigma}^{-} \oplus \mathfrak{l}_{\Sigma} \oplus \mathfrak{u}_{\Sigma}^{+}
$$

da álgebra de Lie de $\mathfrak{g}$. Observamos que se $\Sigma=\emptyset$, então $\mathfrak{p}_{\Sigma}=\mathfrak{b}$, e se $\Sigma=\Pi$, então $\mathfrak{p}_{\Sigma}=\mathfrak{g}$.

Definição 1.2.1 ([CF94]). Seja $V$ um $\mathfrak{l}_{\Sigma}$-módulo simples de peso. Consideramos $V$ como um $\mathfrak{p}_{\Sigma}$-módulo com ação trivial do nilradical $\mathfrak{u}_{\Sigma}^{+}$. O $\mathfrak{g}$-módulo de Verma generalizado $M_{\mathfrak{p}}^{\mathfrak{g}}(\Sigma, V)$ é o módulo induzido

$$
M_{\mathfrak{p}}^{\mathfrak{g}}(\Sigma, V)=\operatorname{Ind}_{\mathfrak{p}}^{\mathfrak{g}} V=U(\mathfrak{g}) \otimes_{U\left(\mathfrak{p}_{\Sigma}\right)} V
$$

Proposição 1.2.2 (Proposição 3.3 em [Fer90]). Se $V$ for um $U\left(\mathfrak{l}_{\Sigma}\right)$-módulo simples, então $M_{\mathfrak{p}}^{\mathfrak{g}}(\Sigma, V)$ terá um único submódulo próprio maximal e um único quociente simples $L_{\mathfrak{p}}^{\mathfrak{g}}(\Sigma, V)$.

Definição 1.2.3 ([Mat00]). Quando $\mathfrak{p} \neq \mathfrak{g}$, o módulo $L_{\mathfrak{p}}^{\mathfrak{g}}(\Sigma, V)$ é chamado induzido parabolicamente. Um g-módulo simples $M$ é cuspidal se for um módulo de peso que não é induzido parabolicamente.

Observação 1.2.4. Escrevemos $M(\lambda)$ para $M_{\mathfrak{b}}^{\mathfrak{g}}\left(\emptyset, \mathbb{C} v_{\lambda}\right)$, e $L(\lambda)$ para $L_{\mathfrak{b}}^{\mathfrak{g}}\left(\emptyset, \mathbb{C} v_{\lambda}\right)$, e serão chamados de módulo de Verma e módulo de peso máximo, respectivamente. Analogamente, no caso $\Sigma=$ 
$\{\alpha\}$, escrevemos $M(\lambda, \mu)$ para $M_{\mathfrak{p}}^{\mathfrak{g}}(\{\alpha\}, V)$ e $L(\gamma, \mu)$ para $L_{\mathfrak{p}}^{\mathfrak{g}}(\{\alpha\}, V)$, para $\alpha \in \Delta$, e chamaremos de módulo induzido de $\mathfrak{s l}_{2}$, em que o elemento de Casimir $c_{\alpha}$ age pelo escalar $\gamma$.

\subsection{Funtor de torção e $\alpha$-Gelfand-Tsetlin módulos}

Recordamos a definição de $\alpha$-Gelfand-Tsetlin módulos para uma álgebra de Lie $\mathfrak{g}$ e descrevemos a construção do funtor de torção $T_{\alpha}$ atribuído a uma raiz positiva $\alpha$ of $\mathfrak{g}$. Assume-se conhecimento básico sobre localização de Ore, como em [MRS01].

\subsubsection{Funtor de torção para álgebras de Lie semi-simples}

Vamos considerar uma álgebra de Lie $\mathfrak{g}$. Para uma raiz positiva $\alpha \in \Delta_{+}$de $\mathfrak{g}$, denotamos por $\mathfrak{s}_{\alpha}$ a subálgebra de Lie de $\mathfrak{g}$ gerada pelo $\mathfrak{s l}_{2}$ triplo $\left(e_{\alpha}, h_{\alpha}, f_{\alpha}\right)$, em que $e_{\alpha} \in \mathfrak{g}_{\alpha}$ e $f_{\alpha} \in \mathfrak{g}_{-\alpha}$ satisfazem $\left[e_{\alpha}, f_{\alpha}\right]=h_{\alpha}$. Além disso, definimos as subálgebras de Lie $\mathfrak{s}_{\alpha}^{+}=\mathfrak{s}_{\alpha} \cap \mathfrak{n}=\mathbb{C} e_{\alpha}$ e $\mathfrak{s}_{\alpha}^{-}=\mathfrak{s}_{\alpha} \cap \overline{\mathfrak{n}}=\mathbb{C} f_{\alpha}$ de $\mathfrak{s}_{\alpha}$. Seja $c_{\alpha}$ o elemento de Casimir quadrático dado por

$$
c_{\alpha}=e_{\alpha} f_{\alpha}+f_{\alpha} e_{\alpha}+\frac{1}{2} h_{\alpha}^{2}
$$

que é um gerador livre do centro $Z\left(\mathfrak{s}_{\alpha}\right)$ de $U\left(\mathfrak{s}_{\alpha}\right)$. Em seguida, denotamos por $\Gamma_{\alpha}$ a subálgebra comutativa de $U(\mathfrak{g})$ gerada pela subálgebra de Cartan $\mathfrak{h}$ e pelo centro $Z\left(\mathfrak{s}_{\alpha}\right)$. Portanto, temos a categoria $\mathcal{H}\left(\mathfrak{g}, \Gamma_{\alpha}\right)$ de $\Gamma_{\alpha}$-Gelfand-Tsetlin módulos, ou simplesmente $\alpha$-Gelfand-Tsetlin módulos, que foram estudados em [FK19a] e em [FK19b].

Como o conjunto $\left\{f_{\alpha}^{n} ; n \in \mathbb{Z}_{\geq 0}\right\}$ de $U(\mathfrak{g})$ é multiplicativo à esquerda (à direita) e dado que $f_{\alpha}$ é um elemento regular localmente ad-nilpotente em $U(\mathfrak{g})$, definimos o funtor de torção (veja Seção $1.1 \mathrm{em}[\mathrm{FK19b}])$ sobre a categoria de $\mathfrak{g}$-módulos de peso.

Definição 1.3.1. Seja $M(\mathfrak{g})$ a categoria de $\mathfrak{g}$-módulos de peso. Para cada módulo $M \in M(\mathfrak{g})$ o funtor de torção

$$
T_{\alpha}=T_{f_{\alpha}}: M(\mathfrak{g}) \rightarrow M(\mathfrak{g})
$$


é definido por

$$
T_{\alpha}(M)=\left(U(\mathfrak{g})_{\left(f_{\alpha}\right)} / U(\mathfrak{g})\right) \otimes_{U(\mathfrak{g})} M \simeq M_{\left(f_{\alpha}\right)} / M
$$

Observemos que o quociente $M_{\left(f_{\alpha}\right)} / M$ significa o quociente de $M_{\left(f_{\alpha}\right)}$ pela imagem do homomorfismo canônico $M \rightarrow M_{\left(f_{\alpha}\right)}$ de $\mathfrak{g}$-módulos. Como $f_{\alpha}$ é um elemento regular localmente ad-nilpotente em $U(\mathfrak{g})$, o funtor $T_{\alpha}$ é exato à direita (veja Proposição 1.5 em [FK19b]). O funtor de torção $T_{\alpha}$ para uma raiz simples $\alpha \in \Pi$ é bem estudado (veja por exemplo [AS03] ou seção 12.6 em [Hum08]). Nesse caso, o funtor $T_{\alpha}$ preserva a categoria $\mathcal{O}(\mathfrak{g})$ a menos de conjugação da ação de $\mathfrak{g}$.

Teorema 1.3.2 (Teorema 3.3 in [FK19b]). Para $\alpha \in \Delta_{+}$, o funtor $T_{\alpha}$ induz o funtor restrito

$$
T_{\alpha}: \mathcal{I}_{f}\left(\mathfrak{g}, \mathfrak{s}_{\alpha}^{+}\right) \rightarrow \mathcal{I}_{f}\left(\mathfrak{g}, \mathfrak{s}_{\alpha}^{-}\right)
$$

onde $\mathcal{I}_{f}\left(\mathfrak{g}, \mathfrak{s}_{\alpha}^{ \pm}\right)$é a categoria de $\mathfrak{g}$-módulos de peso localmente $\mathfrak{s}_{\alpha}^{ \pm}$-finitos finitamente gerados.

Foi mostrado em [FK19a] como o funtor de torção $T_{\alpha}$ pode ser usado para construir g-módulos com $\Gamma_{\alpha}$-multiplicidades finitas. Em particular, temos o seguinte Teorema:

Teorema 1.3.3 (Teorema 3.4 in [FK19b]). Seja $\alpha \in \Delta_{+}$. Se $M$ é um $\mathfrak{g}$-módulo de peso máximo, então $T_{\alpha}(M)$ é um $\mathfrak{g}$-módulo cíclico com $\Gamma_{\alpha}$-multiplicidades finitas.

A seguinte proposição mostra que o funtor de torção preserva o anulador do módulo.

Definição 1.3.4. Para uma álgebra associativa $A$ e um A-módulo $M$, o anulador de $M$ em $A$ é o ideal $\{a \in A \mid$ am $=0$, para todo $m \in M\}$ e será indicado por $\operatorname{Ann}_{A}(M)$.

Proposição 1.3.5 (Proposição 4.3 in [FK20]). Seja $M$ um $\mathfrak{g}$-módulo $U\left(\mathfrak{s}_{\alpha}^{-}\right)$-livre para $\alpha \in \Delta_{+}$. Então temos $\operatorname{Ann}_{U(\mathfrak{g})} T_{\alpha}(M)=\operatorname{Ann}_{U(\mathfrak{g})} M$.

Se $M$ é um g-módulo, então $T_{\alpha}(M)$ é um $\mathfrak{g}$-módulo localmente $\mathfrak{s}_{\alpha}^{-}$-finito para qualquer $\alpha \in \Delta_{+}$. Por outro lado, se $M$ for um $\mathfrak{g}$-módulo localmente $\mathfrak{s}_{\alpha}^{-}$-finito, então $T_{\alpha}(M)=0$. Notemos também que, para qualquer $\alpha \in \Delta_{+}$, o funtor de torção $T_{\alpha}$ comuta com o funtor de translação (veja Teorema $3.6 \mathrm{em}[\mathrm{FK} 19 \mathrm{a}])$.

Seja $\mathfrak{p}=\mathfrak{l} \oplus \mathfrak{u}$ a subálgebra parabólica canônica de $\mathfrak{g}$ associada a um subconjunto $\Sigma$ de $\Pi$ com o nilradical $\mathfrak{u}$, o nilradical oposto $\overline{\mathfrak{u}}$ e a subálgebra de Levi $\mathfrak{l}$. Definimos os subconjuntos

$$
\Delta_{+}^{\mathfrak{u}}=\left\{\alpha \in \Delta_{+} ; \mathfrak{g}_{\alpha} \subset \mathfrak{u}\right\}, \quad \Delta_{+}^{\mathfrak{l}}=\left\{\alpha \in \Delta_{+} ; \mathfrak{g}_{\alpha} \subset \mathfrak{l}\right\}
$$

de $\Delta_{+}$e também

$$
\Lambda^{+}(\mathfrak{p})=\left\{\lambda \in \mathfrak{h}^{*} ;\left\langle\lambda+\rho, \alpha^{\vee}\right\rangle \in \mathbb{Z}_{>0} \text { para todo } \alpha \in \Sigma\right\}
$$

Observação 1.3.6. A condição em $\lambda$ é equivalente a $\left\langle\lambda, \alpha^{\vee}\right\rangle \in \mathbb{Z}_{\geq 0}$, para todo $\alpha \in \Sigma$ (ou todo $\left.\alpha \in \Delta_{+}^{\mathfrak{u}}\right)$. Em particular, $\Lambda^{+}=\Lambda^{+}(\mathfrak{g}) \subset \Lambda^{+}(\mathfrak{p}) \subset \Lambda^{+}(\mathfrak{b})$.

Para um peso $\lambda \in \Lambda^{+}(\mathfrak{p})$, denotamos por $\mathbb{F}_{\lambda}$ o $\mathfrak{p}$-módulo simples de dimensão finita com peso máximo $\lambda$, e por $M_{\mathfrak{p}}^{\mathfrak{g}}(\lambda)$ o g-módulo de Verma generalizado com peso máximo $\lambda$ definido por

$$
M_{\mathfrak{p}}^{\mathfrak{g}}(\lambda)=U(\mathfrak{g}) \otimes_{U(\mathfrak{p})} \mathbb{F}_{\lambda}
$$


Então, para $\alpha \in \Delta_{+}^{\mathfrak{u}}$, definimos o $\alpha$-Gelfand-Tsetlin $\mathfrak{g}$-módulo $W_{\mathfrak{p}}^{\mathfrak{g}}(\lambda, \alpha)$ por

$$
W_{\mathfrak{p}}^{\mathfrak{g}}(\lambda, \alpha)=T_{\alpha}\left(M_{\mathfrak{p}}^{\mathfrak{g}}(\lambda)\right)
$$

Como consequência do Teorema de Poincaré-Birkhoff-Witt, temos os seguintes isomorfismos

$$
M_{\mathfrak{p}}^{\mathfrak{g}}(\lambda) \simeq U(\overline{\mathfrak{u}}) \otimes_{\mathbb{C}} \mathbb{F}_{\lambda} \quad \text { e } \quad W_{\mathfrak{p}}^{\mathfrak{g}}(\lambda, \alpha) \simeq\left(U(\overline{\mathfrak{u}})_{\left(f_{\alpha}\right)} / U(\overline{\mathfrak{u}})\right) \otimes_{\mathbb{C}} \mathbb{F}_{\lambda}
$$

de $U(\overline{\mathfrak{u}})$-módulos. Ademais, como o funtor de torção $T_{\alpha}$ é exato à direita, aplicando $T_{\alpha}$ no homomorfismo sobrejetivo

$$
M_{\mathfrak{b}}^{\mathfrak{g}}(\lambda) \rightarrow M_{\mathfrak{p}}^{\mathfrak{g}}(\lambda)
$$

de módulos de Verma generalizados, obtemos um homomorfismo sobrejetivo

$$
W_{\mathfrak{b}}^{\mathfrak{g}}(\lambda, \alpha) \rightarrow W_{\mathfrak{p}}^{\mathfrak{g}}(\lambda, \alpha)
$$

de $\alpha$-Gelfand-Tsetlin módulos.

Corolário 1.3.7 (Corolário 4.5 em [FK20]). Seja $\lambda \in \Lambda^{+}(\mathfrak{p})$ e $\alpha \in \Delta_{+}^{\mathfrak{u}}$. Então temos

$$
A n n_{U(\mathfrak{g})} W_{\mathfrak{p}}^{\mathfrak{g}}(\lambda, \alpha)=A n n_{U(\mathfrak{g})} M_{\mathfrak{p}}^{\mathfrak{g}}(\lambda)
$$

Definição 1.3.8. Um peso $\lambda \in \mathfrak{h}^{*}$ é chamado antidominante generalizado se $\lambda \in \Lambda^{+}(\mathfrak{p})$ e $\left\langle\lambda+\rho, \alpha^{\vee}\right\rangle \notin \mathbb{Z}_{>0}$, para todo $\alpha \in \Delta_{+}^{\mathfrak{u}}$.

Teorema 1.3.9 (Teorema $9.12 \mathrm{em}\left[\right.$ Hum08]). Se $\lambda$ for antidominante generalizado, então $M_{\mathfrak{p}}^{\mathfrak{g}}(\lambda)$ será simples. Ademais, a recíproca é verdadeira se $\lambda \in \Lambda^{+}(\mathfrak{p})$ for regular.

Observação 1.3.10. Se o módulo de Verma generalizado $M_{\mathfrak{p}}^{\mathfrak{g}}(\lambda)$ for simples, então $M_{\mathfrak{p}}^{\mathfrak{g}}(\lambda) \simeq L_{\mathfrak{b}}^{\mathfrak{g}}(\lambda)$ como $\mathfrak{g}$-módulos.

\subsubsection{Anuladores}

Definição 1.3.11 (Definição 3.1.4 em [Dix77]). Um ideal I de A é primitivo se este for o anulador de um A-módulo simples.

Definição 1.3.12 (Definição 2.6.7 em [Dix77]). Seja $M$ um $\mathfrak{g}$-módulo. Se para cada $z \in Z(\mathfrak{g})$, existe um escalar $\chi(z) \in \mathbb{C}$ tal que $z m=\chi(z) m$ para todo $m \in M$, dizemos que o $\mathfrak{g}$-módulo $M$ admite um caráter central. O mapa $\chi: Z(\mathfrak{g}) \rightarrow \mathbb{C}$ é um homomorfismo de álgebras e é chamado o caráter central do $\mathfrak{g}$-módulo $M$, ou da sua correspondente representação. 
Proposição 1.3.13 (Proposição 2.6.8 em [Dix77]). Todo g-módulo simples admite um caráter central.

Veja os Teoremas 8.4.3, 8.4.4 e 8.5.8 em [Dix77]

Teorema 1.3.14. Denotemos o caráter central do módulo de Verma $M(\lambda)$ por $\chi_{\lambda}$. Então:

a) Se $\lambda \in \mathfrak{h}^{*}$, o anulador de $M(\lambda)$ é o ideal bilateral de $U(\mathfrak{g})$ gerado por Ker $\chi_{\lambda}$; portanto, é o mesmo para todo peso ligado a $\lambda$ e pode ser denotado por $J_{\chi} \operatorname{com} \chi=\chi_{\lambda}$.

b) Para qualquer caráter central $\chi: Z(\mathfrak{g}) \rightarrow \mathbb{C}$, o ideal $J_{\chi}$ é primitivo.

c) Todo ideal primitivo de $U(\mathfrak{g})$ é da forma $\operatorname{Ann}_{U(\mathfrak{g})} L(\lambda)$, para algum módulo de peso máximo simples $L(\lambda), \lambda \in \mathfrak{h}^{*}$; denotaremos esse ideal por $J_{\lambda} \cdot{ }^{2}$

d) Como $\chi$ varia sobre os caracteres centrais, os ideais $J_{\chi}=U(\mathfrak{g})$ Ker $\chi_{\lambda}$ são precisamente os ideais primitivos minimais de $U(\mathfrak{g})$.

e) Suponhamos que $\lambda \in \mathfrak{h}^{*}$ seja dominante. O anulador de $L(\lambda)$ é o único ideal maximal bilateral de $U(\mathfrak{g})$ que contém $U(\mathfrak{g}) \operatorname{Ker} \chi_{\lambda}$.

f) $S e\left\langle\lambda+\rho, \alpha^{\vee}\right\rangle \notin \mathbb{Z} \backslash\{0\}$, para todas as raízes $\alpha$, então $J_{\chi}=J_{\lambda}$.

Definição 1.3.15 (Definição 1.9 em [Hum08]). Seja $S(\mathfrak{h})$ a álgebra de funções polinomiais em $\mathfrak{h}^{*}$.

Veja Teorema 1.10 em [Hum08].

Teorema 1.3.16 (Harish-Chandra). Seja $\psi: Z(\mathfrak{g}) \rightarrow S(\mathfrak{h})$ o homomorfismo torcido de Harish-Chandra.

a) O homomorfismo $\psi$ é um isomorfismo de $Z(\mathfrak{g})$ em $S(\mathfrak{h})^{W}$.

b) Para todo $\lambda, \mu \in \mathfrak{h}^{*}$, temos que $\chi_{\lambda}=\chi_{\mu}$ se, e somente se, $\mu=w \cdot \lambda$ para algum $w \in W$.

c) Todo carácter central $\chi: Z(\mathfrak{g}) \rightarrow \mathbb{C}$ é da forma $\chi_{\lambda}$ para algum $\lambda \in \mathfrak{h}^{*}$.

Teorema 1.3.17 (Teorema 10.2 em [Jos98]). Para todo $\lambda, \mu \in \mathfrak{h}^{*}$. As seguintes condições são equivalentes:

a) $\operatorname{Ann}_{Z(\mathfrak{g})} L(\lambda)=\operatorname{Ann}_{Z(\mathfrak{g})} L(\mu)$;

b) $\mu \in W \cdot \lambda$.

Para estendermos o Teorema anterior para a álgebra envolvente universal, precisamos restringir nosso estudo aos pesos dominantes.

Corolário 1.3.18. Sejam $\lambda, \mu \in \mathfrak{h}^{*}$ pesos dominantes. Se $\mu=w \cdot \lambda$ para algum $w \in W$, então $\operatorname{Ann}_{U(\mathfrak{g})} L(\lambda)=\operatorname{Ann}_{U(\mathfrak{g})} L(\mu)$.

Demonstração. Dado que $\mu=w \cdot \lambda$ para algum $w \in W$, então $\chi_{\lambda}=\chi_{\mu}$, pelo Teorema de HarishChandra. Portanto $A n n_{U(\mathfrak{g})} L(\lambda)=A n n_{U(\mathfrak{g})} L(\mu)$ pela unicidade do anulador (item d) do Teorema 1.3.14), dado que $\lambda$ e $\mu$ são dominantes.

\footnotetext{
${ }^{2}$ Este resultado é conhecido como o Teorema de Duflo (veja [Duf77]).
} 
Se $\lambda$ é dominante regular, a correspondência $I \mapsto I M(\lambda)$ fornece uma injeção que preserva a ordem entre o conjunto de ideais bilaterais de $U(\mathfrak{g})$, contendo $U(\mathfrak{g})$ ker $\chi_{\lambda}$, e submódulos de $M(\lambda)$ (veja os Corolários 4.3 e 4.8 em [Jos79]). Ademais, este mapa é uma bijeção. Assim, temos o seguinte:

Corolário 1.3.19 (Corolário $2.3 \mathrm{em}$ [FHR20]). Dados os pesos $\lambda, \mu \in \mathfrak{h}^{*}$ tais que $\lambda$ seja dominante regular e $\mu$ seja dominante, $A_{U(\mathfrak{g})} L(\lambda)=\operatorname{Ann}_{U(\mathfrak{g})} L(\mu)$ se, e somente se, existe $w \in W$ tal que $\mu=w \cdot \lambda$.

\subsection{Teoria de Mathieu}

Nesta seção apresentamos algumas definições e um resumo dos resultados mais importantes da teoria desenvolvida por Fernando e Mathieu, na classificação dos módulos de peso com espaços de peso de dimensão finita (ver [Fer90] e [Mat00]), os quais são fundamentais para conseguirmos provar os resultados neste trabalho.

\subsubsection{Famílias coerentes}

Em [Mat00], Olivier Mathieu introduziu a noção de família coerente como uma ferramenta fundamental para concluir a classificação de módulos de peso simples com espaços de peso de dimensão finita sobre $\mathfrak{g}$. Fixada uma subálgebra de Cartan $\mathfrak{h} \subseteq \mathfrak{g}$. Seja $\operatorname{supp} M \subseteq \mathfrak{h}^{*}$ o suporte (o conjunto de pesos) do $\mathfrak{g}$-módulo $M$ e denotemos por $M(\mu)$ o espaço de pesos de $M$ correspondente ao peso $\mu \in \mathfrak{h}^{*}$. Ademais, denotaremos por $U(\mathfrak{g})^{\mathfrak{h}}:=\{x \in U(\mathfrak{g}) \mid[x, \mathfrak{h}]=0\}$ o centralizador de $\mathfrak{h}$ na álgebra envolvente universal $U(\mathfrak{g})$. A definição de Mathieu (veja seção 4 em [Mat00]) é então a seguinte:

Definição 1.4.1. Uma família coerente de $\mathfrak{g}$-módulos é um $\mathfrak{g}$-módulo de pesos $\mathcal{C}$ para o qual:

- Existe $d \in \mathbb{Z}_{>0}$, chamado grau de $\mathcal{C}$, de modo que $\operatorname{dim} \mathcal{C}(\mu)=d$ para todo $\mu \in \mathfrak{h}^{*}$ no suporte do módulo $\mathcal{C}$.

- Dado qualquer $U \in U(\mathfrak{g})^{\mathfrak{h}}$, a função que leva $\mu \in \mathfrak{h}^{*}$ a $\operatorname{tr}_{\mathcal{C}(\mu)} U$ é polinomial em $\mu$.

Outra importante definição é a de módulos limitados: 


\section{Definição 1.4.2.}

- Um l-módulo limitado é um módulo de peso de dimensão infinita para o qual existe um limite superior (finito) nas multiplicidades (as dimensões dos espaços de peso). A multiplicidade máxima é chamada de grau do l-módulo.

- O suporte essencial ess-supp $\mathcal{N}$ de um $\mathfrak{l}$-módulo $\mathcal{N}$ é o conjunto de pesos cujas multiplicidades são maximais.

Observamos que Mathieu chama um módulo de peso com multiplicidades uniformemente limitadas de admissível. Preferimos não usar essa terminologia, pois ela entra em conflito com nosso objetivo, no qual utilizamos a terminologia amplamente usada no estudo de álgebras de vertex afins e seus módulos (veja [KW88]).

Em particular, o suporte de uma família coerente é todo $\mathfrak{h}^{*}$. Mais especificamente, temos a seguinte proposição:

Proposição 1.4.3 (Corolário 1.4 em [Mat00]). Para um g-módulo de peso $M$ com espaços de peso finitos, as seguintes asserções são equivalentes:

a) $M$ é cuspidal.

b) $e_{\alpha}$ age injetivamente em $M$, para cada $\alpha \in \Delta$.

c) O suporte de $M$ é exatamente uma $Q$-coclasse, isto é, o $\operatorname{supp}(\mathcal{N})=\lambda+\mathrm{Q}$, por algum $\lambda \in \mathfrak{h}^{*}$.

Observação 1.4.4. Um módulo que satisfaça b) na Proposição anterior é chamado livre de torção, e um módulo com a propriedade c) é chamado denso.

Proposição 1.4.5 (Corolário 1.5 em [Mat00]). Seja M um g-módulo cuspidal com espaços de peso de dimensão finita. Temos então o seguinte:

a) Para qualquer $\alpha \in \Delta, f_{\alpha}$ age bijetivamente em $M$.

b) Existe um número inteiro d tal que $\operatorname{dim} M_{\lambda}=d$, para qualquer $\lambda \in S u p p M$.

Proposição 1.4.6 (Lema 3.3 em [Mat00]). Qualquer g-módulo limitado com suporte em uma única $Q$-coclasse tem comprimento finito.

Uma família coerente $\mathcal{C}$ de $\mathfrak{l}$-módulos é, portanto, altamente redutível em geral, a qual é decomposta como

$$
\mathcal{C} \cong \bigoplus_{\lambda \in \operatorname{supp} \mathcal{C} / Q_{\curlywedge}} \mathcal{C}_{\lambda}
$$

em que $Q_{\mathfrak{l}}$ indica o reticulado de raízes de $\mathfrak{l}$. Se pelo menos um $\operatorname{dos} \mathcal{C}_{\lambda}$ for simples, então a família coerente $\mathcal{C}$ será irredutível e, se todos os $\mathcal{C}_{\lambda}$ forem l-módulos semissimples, então a família coerente será chamada semissimples.

Um exemplo é $\mathfrak{l}=\mathfrak{s l}_{2}$ para o qual $U(\mathfrak{l})^{\mathfrak{h}}$ é o anel polinomial gerado por $\mathfrak{h}$ e o centro $U(\mathfrak{l})^{\mathfrak{l}}$, sendo este último um polinômio quadrático no Casimir $c$. A classificação de módulos de pesos simples (com espaços de pesos de dimensão finita) é, portanto, elementar; veja [Maz10, Thm. 3.32] por exemplo. De fato, um módulo de peso simples é de peso máximo, mínimo ou denso. Os somandos 
$\mathcal{C}_{\lambda}$ de uma família coerente e semissimples irredutível $\mathcal{C}$ sobre $\mathfrak{s l}_{2}$ são, portanto, somas diretas de módulos simples de peso máximo e peso mínimo ou módulos densos simples. Observemos que o Casimir $c$ age como uma constante em cada soma simples de $\mathcal{C}$, pelo lema de Schur, portanto, ele deve agir como uma constante em todos os $\mathcal{C}$ para agir polinomialmente.

Antes de continuar, lembramos alguns termos necessários.

Definição 1.4.7. Uma álgebra de Lie é redutiva se for uma soma direta de uma álgebra de Lie semissimples e uma álgebra de Lie abeliana. Diz-se que uma álgebra de Lie redutiva de dimensão finita é do tipo $\boldsymbol{A C}$ se seus ideais simples forem todos dos tipos A e C.

Recordamos que o tipo de uma álgebra de Lie simples de dimensão finita se refere ao nome dado ao seu diagrama de Dynkin. Portanto, $\mathfrak{s l}_{n}$ é do tipo A, enquanto $\mathfrak{s p}_{2 n}$ é do tipo C, para todo $n \in \mathbb{Z}_{\geq 2}$. Proposição 1.4.8 (Teorema 5.2 e Observação 5.4 em [Fer90]). Uma álgebra de Lie redutiva $\mathfrak{l}$ de dimensão finita admite um módulo denso simples se, e somente se, for do tipo AC.

Apesar disso, famílias coerentes fornecem os meios para construir e entender módulos de peso simples com espaços de peso de dimensão finita (veja 1.4.11).

Proposição 1.4.9 (Teorema 4.21 em [Fer90]). Seja g uma álgebra de Lie redutiva de dimensão finita. Todo g-módulo finitamente gerado com espaços de peso de dimensão finita possui comprimento finito.

Teorema 1.4.10 (Teorema $5.2 \mathrm{em}$ [Fer90]). Uma álgebra $\mathfrak{g}$ admite um módulo denso simples se, e somente se, for do tipo A ou do tipo $C$.

Não surpreende que subálgebras parabólicas sejam importantes ao classificar módulos de peso simples. Para isso, o seguinte resultado é pertinente.

Teorema 1.4.11 (Teorema 4.18 em [Fer90]). Todo g-módulo simples com espaços de peso de dimensão finita é induzido parabolicamente ou cuspidal.

Observamos que se a subálgebra parabólica é uma subálgebra de Borel (então $\mathfrak{l}=\mathfrak{h}$ ), todos os l-módulos simples são densos e a indução parabólica resulta em g-módulos de peso máximo. Desse modo, a indução parabólica é igual ao funtor identidade se $\mathfrak{l}=\mathfrak{g}$.

Concluímos esta seção citando alguns resultados fundamentais para famílias coerentes, cujas provas podem ser encontradas no artigo de Mathieu [Mat00].

Proposição 1.4.12 ([Mat00]). Seja l uma álgebra de Lie redutiva não abeliana de dimensão finita. Então:

a) [Proposição 4.8i] Todo l-módulo limitado é mergulhado numa única família coerente semisimples irredutivel.

b) [Proposição 4.8ii] Todo submódulo de dimensão infinita de uma familia coerente irredutível de grau d é limitado e seu grau também é d.

c) [Lema 5.3ii] Famílias coerentes existem se, e somente se, $\mathfrak{l}$ for do tipo AC (compare com 1.4.8).

d) [Proposição 5.7] Dada uma família coerente e semissimples irredutivel, existe uma escolha de subálgebra Borel para $\mathfrak{l}$, de modo que a familia contém um módulo de peso máximo limitado simples. 


\subsubsection{Famílias coerentes de álgebras de Lie simples do tipo AC}

Nesta seção, lembramos a classificação explícita das famílias coerentes semissimples irredutíveis sobre $\mathfrak{g}$, dada por Mathieu em [Mat00]. Lembrando que não há famílias coerentes se $\mathfrak{g}$ não for do tipo A ou C (veja Teorema 1.4.10). Ressaltamos ainda que neste trabalho somente descreveremos a família do tipo A, uma vez que nosso objetivo é realizar módulos sobre álgebras de vertex de tipo A.

Escolhamos uma subálgebra de Borel de $\mathfrak{g}$. Denotaremos por $\mathcal{B}_{\mathfrak{g}}$ o conjunto de pesos $\lambda \in \mathfrak{h}^{*}$ de modo que o g-módulo de peso máximo simples de $L(\lambda)$ seja limitado. Como famílias semissimples e coerentes são invariantes sob a ação do grupo de Weyl (ver Proposição 6.2 em [Mat00]), não importa a subálgebra de Borel que escolhemos. Observamos que o conjunto $\mathcal{B}_{\mathfrak{g}}$ é vazio se $\mathfrak{g}$ não for do tipo A ou C (Proposição 4.8 e Lema 5.3 em [Mat00]).

\subsubsection{Tipo A}

Seja $\mathfrak{g}=\mathfrak{s l}_{n+1}$, com $n \geq 1$. Com relação à subálgebra de Borel escolhida, temos as raízes simples $\alpha_{1}, \ldots, \alpha_{n}$, a raiz longa $\theta$, o peso $\rho$ e os pesos integrais dominantes $\Delta^{+}$. Para cada $\lambda \in \mathfrak{h}^{*}$, definimos

$$
A(\lambda)=\left\{i \in\{1, \ldots, n\}:\left\langle\lambda+\rho, \alpha_{i}^{\vee}\right\rangle \notin \mathbb{Z}_{>0}\right\},
$$

em que a forma de Killing é normalizada por $(\theta, \theta)=2$. Observamos que $A(\lambda)=\varnothing$ se, e somente se, $\lambda \in \Delta^{+}$.

Proposição 1.4.13 (Lema 8.1 e Proposição 8.5 em [Mat00]). Para $\mathfrak{g}=\mathfrak{s l}_{n+1}$, o conjunto $\mathcal{B}=\mathcal{B}_{\mathfrak{g}}$ consiste dos elementos $\lambda \in \mathfrak{h}^{*}$ que satisfaçam pelo menos uma das seguintes condições:

a) $A(\lambda)=\{1\}$ ou $\{n\}$.

b) $A(\lambda)=\{i\}$, com $1<i<n$, e $\left\langle\lambda+\rho, \alpha_{i}^{\vee}+\alpha_{i-1}^{\vee}\right\rangle \in \mathbb{Z}_{>0}$ ou $\left\langle\lambda+\rho, \alpha_{i}^{\vee}+\alpha_{i+1}^{\vee}\right\rangle \in \mathbb{Z}_{>0}$.

c) $A(\lambda)=\{i, i+1\}$, com $1 \leq i<n, e\left\langle\lambda+\rho, \alpha_{i}^{\vee}+\alpha_{i+1}^{\vee}\right\rangle \in \mathbb{Z}_{>0}$.

Por exemplo, apenas a) se aplica quando $\mathfrak{g}=\mathfrak{s l}_{2}$ e, portanto, $\mathcal{B}_{\mathfrak{s l}_{2}}=\mathfrak{h}^{*} \backslash \Delta^{+}\left(\mathfrak{s l}_{2}\right)$ é o conjunto de pesos cuja etiqueta de Dynkin não é um número inteiro não negativo. Para $\mathfrak{g}=\mathfrak{s l}_{3}$, b) não se aplica e $\mathcal{B}_{\mathfrak{s l}_{3}}$ é a união de dois conjuntos: um composto pelos pesos que possuem precisamente uma etiqueta de Dynkin inteira não negativa, e o outro consiste em pesos sem etiquetas de Dynkin inteiros não negativos, mas cuja soma das etiquetas de Dynkin se encontra em $\mathbb{Z}_{\geq-1}$.

Para $\lambda, \mu \in \mathcal{B}$, escrevemos $\lambda \rightarrow \mu$ se existir $i \in A(\lambda) \operatorname{com} \mu=s_{i} \cdot \lambda$. 
Proposição 1.4.14 (Teorema $8.6 \mathrm{em}$ [Mat00]). Existe uma correspondência bijetiva entre o conjunto de (classes de equivalência de) famílias coerentes semissimples irredutíveis de $\mathfrak{s l}_{n+1}$-módulos e o conjunto $\mathcal{B} /(\rightarrow)$ de componentes conexas em $\mathcal{B}$. Essa correspondência envia uma família coerente semissimples irredutivel $\mathcal{C}$ ao conjunto

$$
\left\{\lambda \in \mathfrak{h}^{*}: \lambda \notin \Delta^{+} \text {e } \mathcal{L}_{\lambda} \subset \mathcal{C}\right\} \in \mathcal{B} /(\rightarrow)
$$

dos pesos máximos dos submódulos de peso máximo de dimensão infinita de $\mathcal{C}$.

Isso mostra que as famílias coerentes e semissimples irredutíveis dos $\mathfrak{s l}_{n+1}$-módulos são completamente caracterizadas por seus submódulos limitados de peso máximo (e, de fato, um único representante o fará). Como todo elemento de $U(\mathfrak{g})^{\mathfrak{h}}$ age polinomialmente em uma determinada família coerente $\mathcal{C}$, segue-se que cada elemento do centro $U(\mathfrak{g})^{\mathfrak{h}}$, isto é cada operador de Casimir, age como uma constante em $\mathcal{C}$. Em outras palavras, $\mathcal{C}$ tem um caráter central definido. Portanto, é natural perguntar se o caráter central também caracteriza completamente uma família coerente semissimples irredutível. Dado $\lambda \in \mathcal{B}$, a pergunta anterior equivale a decidir se $(W \cdot \lambda) \cap \mathcal{B}$ tem uma única componente conexa em $\mathcal{B}$ ou não.

Proposição 1.4.15 (Lema 8.3 em [Mat00]).

a) Se $\lambda \in \mathcal{B}$ é um peso integral, então a componente conexa $[\lambda] \in \mathcal{B} /(\rightarrow)$ tem $n$ elementos. Caso contrário, $[\lambda]$ tem $n+1$ elementos.

b) A intersecção $(W \cdot \lambda) \cap \mathcal{B}$ é a única componente conexa em $\mathcal{B}$, a menos que $\lambda$ seja regular $e$ integral; neste caso, é a união de $n$ componentes conexas.

Concluímos que uma família coerente e semissimples irredutível de $\mathfrak{s l}_{n+1}$ - módulos é completamente caracterizada por seu caráter central, a menos que seus submódulos de peso máximo alterem os pesos máximos integrais regulares (e se um faz, então todos eles fazem).

Ilustramos essas ideias para $\mathfrak{g}=\mathfrak{s l}_{2}$. Neste caso, as componentes conexas de $\mathcal{B}_{\mathfrak{s l}_{2}}=\mathfrak{h}^{*} \backslash \Delta^{+}\left(\mathfrak{s l}_{2}\right)$ têm o formato $[\lambda]=\{\lambda\}$, se $\lambda$ for integral, e $[\lambda]=\left\{\lambda, s_{1} \cdot \lambda\right\}$ caso contrário. O conjunto de componentes conexas de $\mathcal{B}_{\mathfrak{s l}_{2}}$ decompõe-se em pesos integrais regulares, integrais singulares e não integrais do seguinte modo:

$$
\mathcal{B}_{\mathfrak{s} \mathfrak{l}_{2}} /(\rightarrow)=\left(\bigcup_{\lambda \in \mathbb{Z}_{\leq-2}}\left\{\lambda \omega_{1}\right\}\right) \cup\left(\left\{-\omega_{1}\right\}\right) \cup\left(\bigcup_{\lambda \in \mathbb{C} \backslash \mathbb{Z}}\left\{\lambda \omega_{1},-(\lambda+2) \omega_{1}\right\}\right) .
$$

Ademais, o caráter central sempre caracteriza completamente as famílias coerentes. Embora existam pesos integrais regulares deslocados em $\mathcal{B}_{\mathfrak{s l}_{2}}$ (aqueles com $\lambda \in \mathbb{Z}_{\leq-2}$ ), as $W$-órbitas (parcial) $(W \cdot \lambda) \cap \mathcal{B}_{\mathfrak{s l}_{2}}=\{\lambda\}$ coincidem com as componentes conexas neste caso, consistente com a Proposição 1.4.15 (uma vez que $n=1$ ). 


\subsubsection{Localização de módulos de peso}

Recordamos a definição do funtor de localização nos $U(\mathfrak{g})$-módulos. Para detalhes sobre essa localização, veja [Gra14] ou a Seção $04 \mathrm{em}$ [Mat00]. Nesta seção, $F:=\left\{f_{1}, \ldots, f_{r}\right\}$ indicará qualquer conjunto de elementos comutativos localmente adnilpotentes de $U(\mathfrak{g})$.

Definição 1.4.16. Um $U(\mathfrak{g})$-módulo será chamado $\boldsymbol{F}$ injetivo (respectivamente, $\boldsymbol{F}$ bijetivo) se cada $f \in F$ agir injetivamente (respectivamente, bijetivamente) em $M$.

Observação 1.4.17. Se $M$ for simples e os seus espaços de peso têm dimensão finita, a condição de que $f_{\alpha}$ age injetivamente (respectivamente, bijetivamente) em $M$ é equivalente à condição em que o operador $f_{\alpha}: M^{\lambda} \rightarrow M^{\lambda-\alpha}$ é injetivo (respectivamente, bijetivo) em que $\lambda \in \operatorname{supp} M$, veja Corolário 3.4 em [DMP00].

\section{Localização torcida}

Por $D_{F} U(\mathfrak{g})$, denotamos a localização de $U(\mathfrak{g})$ em relação ao conjunto multiplicativo $\langle F\rangle$ gerado por $F$. Da mesma forma, para um $\mathfrak{g}$-módulo $M$, indicamos por $D_{F} M=D_{F} U(\mathfrak{g}) \otimes_{U(\mathfrak{g})} M$ a localização de $M$ em relação a $\langle F\rangle$. Vamos considerar $D_{F} M$ como um $U(\mathfrak{g})$-módulo e como um $D_{F} U(\mathfrak{g})$-módulo.

Para $\mathbf{x}=\left(x_{1}, \ldots, x_{r}\right) \in \mathbb{C}^{r}$, consideramos o automorfismo $\Theta_{F}^{\mathbf{x}}$ (chamado de conjugação generalizada) de $D_{F} U(\mathfrak{g})$ dado por

$$
\Theta_{F}^{\mathbf{x}}(u):=\sum_{i_{1}, \ldots, i_{r} \geq 0}\left(\begin{array}{c}
x_{1} \\
i_{1}
\end{array}\right) \ldots\left(\begin{array}{c}
x_{r} \\
i_{r}
\end{array}\right) \operatorname{ad}\left(f_{1}\right)^{i_{1}} \ldots \operatorname{ad}\left(f_{r}\right)^{i_{r}}(u) f_{1}^{-i_{1}} \ldots f_{r}^{-i_{r}},
$$

para $u \in D_{F} U(\mathfrak{g})$, em que $\left(\begin{array}{c}x \\ i\end{array}\right):=x(x-1) \ldots(x-i+1) / i$ !, para $x \in \mathbb{C}, i \in \mathbb{Z}_{>0}$ e $\left(\begin{array}{l}x \\ 0\end{array}\right):=1$ ([Mat00, Seção 4]).

Observação 1.4.18. Como alternativa, a conjugação generalizada pode ser descrita como:

$$
\Theta_{F}^{\mathbf{x}}(u):=\prod_{j=1}^{k} \Theta_{f_{j}}^{x_{j}}(u)
$$

em que $\Theta_{f}^{x}(u):=\sum_{i \geq 0}\left(\begin{array}{c}x \\ i\end{array}\right)(\operatorname{ad} f)^{i}(u) f^{-i}$

Definição 1.4.19. Dado um $D_{F} U(\mathfrak{g})$-módulo $N$, indicaremos por $\Phi_{F}^{\mathbf{x}} N$ o $D_{F} U(\mathfrak{g})$-módulo $N$ torcido por $\Theta_{F}^{\mathbf{x}}$, em que a nova ação (chamada ação torcida) é dada por

$$
u \cdot v^{\mathbf{x}}:=\left(\Theta_{F}^{\mathbf{x}}(u) \cdot v\right)^{\mathbf{x}},
$$


para $u \in D_{F} U(\mathfrak{g}), v \in N$. Aqui $v^{\mathbf{x}}$ representa o elemento $v$ considerado como um elemento de $\Phi_{F}^{\mathbf{x}} N$.

Definição 1.4.20. Se $M$ é um $\mathfrak{g}$-módulo $e \mathrm{x} \in \mathbb{C}^{r}$, então $D_{F}^{\mathrm{x}} M:=\Phi_{F}^{\mathrm{x}} D_{F} M$ é a localização torcida de $M$ em relação a $F$ e x.

Observação 1.4.21. Observamos que, para $\mathbf{x} \in \mathbb{Z}^{r}$, temos $\Theta_{F}^{\mathbf{x}}(u)=\mathbf{f}^{\mathbf{x}} u \mathbf{f}^{-\mathbf{x}}$, em que $\mathbf{f}^{\mathbf{x}}:=f_{1}^{x_{1}} \ldots f_{r}^{x_{r}}$. Ademais, $D_{F}^{\mathbf{x}} M$ e $D_{F} M$ são isomorfos para qualquer $\mathfrak{g}$-módulo $M$.

Proposição 1.4.22 (Proposição 3.1 em [FHR20]). Seja $M$ um $U(\mathfrak{g})$-módulo F-bijetivo, $L, N$ submódulos de $M$, tais que $L$ não seja F-bijetivo, e $M / L$ seja simples. Se $L$ é isomorfo a um submódulo de $D_{F} N$, então $D_{F} N \simeq M$.

\section{Localização torcida para $\mathfrak{s l}_{n+1}$-módulos com espaços de peso de dimensão finita}

Neste caso, consideramos $\mathfrak{g}=\mathfrak{s l}_{n+1}$. Lembramos que um subconjunto $\Omega$ de $\Delta$ é um conjunto de raízes comutativas se $\alpha, \beta \in \Omega$ implicar $\left[e_{\alpha}, e_{\beta}\right]=0$. Neste trabalho, os conjuntos multiplicativos sempre terão o formato $F=\left\langle e_{\alpha} \mid \alpha \in \Omega\right\rangle$, em que $\Omega$ é um conjunto de $k$ raízes comutativas, e $e_{\alpha}$ está no espaço de $\alpha$-raiz de $\mathfrak{s l}_{n+1}$.

Os conjuntos maximais (com relação à inclusão) de raízes comutativas podem ser parametrizados pelo conjunto $\mathcal{P}$ de subconjuntos próprios não vazios de $\{0,1, \ldots, n\}$. De fato, para $J=\left\{i_{1}, \ldots, i_{k}\right\}$ em $\mathcal{P}$,

$$
\Lambda_{J}:=\left\{\varepsilon_{i}-\varepsilon_{j} \mid i \in J, j \notin J\right\}
$$

é um conjunto máximo de raízes comutativas.

A seguir, será apresentada uma classificação dos g-módulos limitados simples em termos de localização torcida dos módulos de peso máximo (veja [Mat00]).

Teorema 1.4.23 (Teorema 13.3 em [Mat00]). Todo módulo cuspidal é isomorfo a $D_{F}^{\mathbf{x}} L(\lambda)$, para algum módulo limitado de peso máximo simples $L(\lambda)$, um conjunto $F$ de $r$ raízes comutativas $e$ $x \in \mathbb{C}^{r}$. Ademais, os caracteres centrais de $M$ e $L(\lambda)$ coincidem, $\operatorname{deg} M=\operatorname{deg} L(\lambda) e$

$$
\operatorname{Supp} M=x_{1} \alpha_{1}+\cdots+x_{r} \alpha_{r}+\operatorname{Supp} L(\lambda)+\mathbb{Z} \Sigma \text {. }
$$

Uma propriedade importante da localização torcida é que ela preserva os anuladores em $U\left(\mathfrak{s l}_{n+1}\right)$.

Lema 1.4.24 (Lema 2.8 em [GP20]). Sejam $M$ um $U\left(\mathfrak{s l}_{n+1}\right)$-módulo com espaços de pesos de dimensão finita, e $D_{F}^{\mathbf{x}} M$ a localização torcida de $M, \mathbf{x} \in \mathbb{C}^{k}$. Então,

$$
A n n_{U\left(\mathfrak{s l}_{n+1}\right)} M \subset A n n_{U\left(\mathfrak{s l}_{n+1}\right)} D_{F}^{\mathbf{x}} M .
$$

Como consequência do Lema anterior, temos o seguinte: 
Corolário 1.4.25 (Corolário 2.9 em [GP20]). Seja $M$ um $\mathfrak{s l}_{n+1}$-módulo simples limitado de dimensão infinita. Existe então um módulo de peso máximo simples limitado L, tal que $A n n_{U\left(\mathfrak{s l}_{n+1}\right)} M=$ $A n n_{U\left(\mathfrak{s l}_{n+1}\right)} D_{F}^{\mathrm{x}} L=A n n_{U\left(\mathfrak{s l}_{n+1}\right)} L$.

Proposição 1.4.26 (Proposição 3.4 em [FHR20]). Seja $M$ um g-módulo de peso, com espaços de peso de dimensão finita, e suponhamos que $F$ é injetivo em M. Então,

$$
A n n_{U(\mathfrak{g})} M=A n n_{U(\mathfrak{g})} D_{F} M
$$

para qualquer $\mathbf{x} \in \mathbb{C}^{r}$. Ademais, Ann $n_{U(\mathfrak{g})} M \subset A n n_{U(\mathfrak{g})} N$, para qualquer subquociente $N$ de $D_{F}^{\mathbf{x}} M$. Demonstração. Como $M$ é um submódulo de $D_{F} M, A n n_{U\left(\mathfrak{s l}_{n+1}\right)} D_{F} M \subset A n n_{U\left(\mathfrak{s l}_{n}\right)} M$. A outra inclusão segue do Lema 1.4.24 para $\mathbf{x}=0$.

Agora, suponhamos que $N$ seja um subquociente de $D_{F}^{\mathbf{x}} M$, logo existem submódulos $W$ e $W^{\prime}$ de $D_{F}^{\mathbf{x}} M$ tal que $N=\frac{V}{V^{\prime}}$. Assim temos que $A n n_{U\left(\mathfrak{s i}_{n+1}\right)} D_{F}^{\mathbf{x}} M \subset A n n_{U\left(\mathfrak{s i}_{n+1}\right)} N$, de fato, para todo $u \in V$ e para qualquer $a \in A n n_{U\left(\mathfrak{s l}_{n+1}\right)}\left(D_{F}^{\mathbf{x}} M\right)$, temos que $a \cdot\left(u+V^{\prime}\right)=(a \cdot u)+V^{\prime}=0+V^{\prime}=V^{\prime}$. Finalmente, pela aplicação do Lema 1.4.24, obtemos o resultado.

Corolário 1.4.27 (Corolário 3.5 em [FHR20]). Sejam $\lambda \in \mathfrak{h}^{*}$ dominante, $\mathbf{x} \in \mathbb{C}^{r}$ e F injetivo em $L(\lambda)$. Se $N \neq 0$ for um subquociente simples de $D_{F}^{\mathbf{x}} L(\lambda)$, então

$$
A n n_{U(\mathfrak{g})} L(\lambda)=A n n_{U(\mathfrak{g})} D_{F}^{\mathbf{x}} L(\lambda)=A n n_{U(\mathfrak{g})} N .
$$

Demonstração. No Lema 1.4.24, temos que

$$
A n n_{U(\mathfrak{g})} L(\lambda) \subset A n n_{U(\mathfrak{g})} D_{F}^{\mathbf{x}} L(\lambda) \subset A n n_{U(\mathfrak{g})} N \neq U(\mathfrak{g}) .
$$

Por outro lado, $A n n_{U(\mathfrak{g})} L(\lambda)$ é um ideal maximal, portanto o resultado segue da Proposição 1.3.14. 


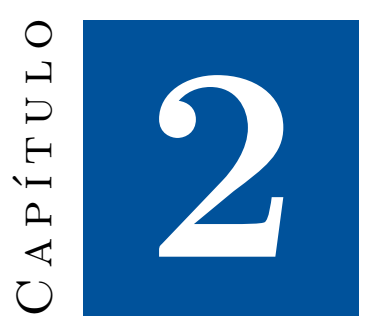

\section{Álgebras de vertex}

Neste capítulo introduziremos conceitos preliminares e algumas propriedades dos módulos e álgebras de vertex. Começaremos retomando alguns conceitos sobre as álgebras de Kac-Moody afim, seguindo com a definição de álgebras de vertex e as suas representações. Na sequência, apresentaremos algumas definições e teoremas relativos à álgebra de Zhu, tais como o funtor de Zhu e o teorema da correspondência entre álgebras de vertex e álgebras de Zhu. Posteriormente, estudaremos os conceitos relacionados as álgebras de vertex afim universal, para as quais são definidas as chamadas representações admissíveis e que foram classificadas por Arakawa em [Ara16]. Encerraremos este capítulo com as definições e resultados relativos às órbitas nilpotentes, tais como a existência de uma única órbita nilpotente cujo fecho é igual à variedade associada a um ideal de $\mathfrak{g}$, para cada número admissível $k$. Em particular, nosso interesse será estudar $\mathfrak{s l}_{n+1}$-módulos admissíveis de nível $k$ nas órbitas minimal e principal.

A notação, assim como a maioria das construções deste capítulo, seguem [Kac98, Kac17, Kac90, FB04].

\section{1 Álgebras de Kac-Moody afim}

Seja $\kappa$ uma forma bilinear simétrica $\mathfrak{g}$-invariante em $\mathfrak{g}$. A álgebra de Kac-Moody afim $\widehat{\mathfrak{g}}_{\kappa}$ associada a $\mathfrak{g}$ de nível $\kappa$ é a extensão central 1-dimensional $\widehat{\mathfrak{g}}_{\kappa}=\mathfrak{g}((t)) \oplus \mathbb{C} c$ da álgebra de loop formal $\mathfrak{g}((t))=\mathfrak{g} \otimes_{\mathbb{C}} \mathbb{C}((t))$, com as relações de comutação

$$
[a \otimes f(t), b \otimes g(t)]=[a, b] \otimes f(t) g(t)-\kappa(a, b) \operatorname{Res}_{t=0}(f(t) d g(t)) c
$$


em que $c$ é o elemento central de $\widehat{\mathfrak{g}}_{\kappa}, a, b \in \mathfrak{g}$ e $f(t), g(t) \in \mathbb{C}((t))$. Observamos que as álgebras de Lie $\widehat{\mathfrak{g}}_{\kappa}$ e $\widehat{\mathfrak{g}}_{\kappa^{\prime}}$ para formas bilineares simétricas $\mathfrak{g}$-invariantes $\kappa$ e $\kappa^{\prime}$ em $\mathfrak{g}$ são isomorfas se $\kappa^{\prime}=k \kappa$ por algum $k \in \mathbb{C} \backslash\{0\}$. Introduzindo a notação $a_{n}=a \otimes t^{n}$ para $a \in \mathfrak{g}$ e $n \in \mathbb{Z}$, as relações de comutação (2.1) podem ser simplificadas na forma

$$
\left[a_{m}, b_{n}\right]=[a, b]_{m+n}+m \kappa(a, b) \delta_{m,-n} c
$$

$\operatorname{com} m, n \in \mathbb{Z}$ e $a, b \in \mathfrak{g}$.

Dada uma subálgebra de Cartan $\mathfrak{h}$ de $\mathfrak{g}, \widehat{\mathfrak{h}}=\left(\mathfrak{h} \otimes_{\mathbb{C}} \mathbb{C} 1\right) \oplus \mathbb{C} c$ é uma subálgebra de Cartan de $\widehat{\mathfrak{g}}_{\kappa}$. Vale observar que, enquanto duas subálgebras de Borel de $\mathfrak{g}$ são conjugadas por um automorfismo de $\mathfrak{g}$, quaisquer duas subálgebras de Borel de $\widehat{\mathfrak{g}}_{\kappa}$ podem não ser conjugadas por um automorfismo de $\widehat{\mathfrak{g}}_{\kappa}$ (a descrição completa das classes de equivalência de subálgebras de Borel e parabólicas é dada nos Teoremas 2.4, 2.5 e 2.6 em [Fut97]).

Seja $\mathfrak{p}$ uma subálgebra parabólica padrão de $\mathfrak{g}$ com o nilradical $\mathfrak{u}$, o nilradical oposto $\overline{\mathfrak{u}}$ e a subálgebra de Levi l. Então a subálgebra parabólica $\widehat{\mathfrak{p}}_{\mathrm{st}}$ de $\widehat{\mathfrak{g}}_{\kappa}$ associada a $\mathfrak{p}$ é dada por

$$
\widehat{\mathfrak{p}}_{\mathrm{st}}=\widehat{\mathfrak{l}}_{\mathrm{st}} \oplus \widehat{\mathfrak{u}}_{\mathrm{st}}
$$

em que a subálgebra de Levi $\widehat{\mathfrak{l}}_{\mathrm{st}}$ é definida por

$$
\widehat{\mathfrak{l}}_{\mathrm{st}}=(\mathfrak{l} \otimes \mathbb{C}) \mathbb{C} 1 \oplus \mathbb{C} c
$$

e o nilradical $\widehat{\mathfrak{u}}_{\text {st }}$ e o nilradical oposto $\widehat{\overline{\mathfrak{u}}}_{\text {st }}$ por

$$
\widehat{\mathfrak{u}}_{\mathrm{st}}=\left(\mathfrak{u} \otimes_{\mathbb{C}} \mathbb{C} 1\right) \oplus\left(\mathfrak{g} \otimes_{\mathbb{C}} t \mathbb{C}[[t]]\right) \quad \text { and } \quad \widehat{\overline{\mathfrak{u}}}_{\mathrm{st}}=\left(\overline{\mathfrak{u}} \otimes_{\mathbb{C}} \mathbb{C} 1\right) \oplus\left(\mathfrak{g} \otimes_{\mathbb{C}} t^{-1} \mathbb{C}\left[t^{-1}\right]\right)
$$

Além disso, temos a decomposição triangular correspondente

$$
\widehat{\mathfrak{g}}_{\kappa}=\widehat{\overline{\mathfrak{u}}}_{\mathrm{st}} \oplus \widehat{\mathfrak{l}}_{\mathrm{st}} \oplus \widehat{\mathfrak{u}}_{\mathrm{st}}
$$

da álgebra de Lie $\widehat{\mathfrak{g}}_{\kappa}$. Se $\mathfrak{p}=\mathfrak{b}$ então $\widehat{\mathfrak{b}}_{\text {st }}$ é a subálgebra de Borel padrão e se, $\mathfrak{p}=\mathfrak{g}$, então $\widehat{\mathfrak{g}}_{\mathrm{st}}$ é a subálgebra parabólica maximal padrão. Para subálgebras parabólicas mais gerais da álgebra de Kac-Moody afim $\widehat{\mathfrak{g}}_{\kappa}$, veja [FK18].

Com o propósito de descrever a estrutura do sistema de raízes da álgebra afim de Kac-Moody $\widehat{\mathfrak{g}}_{\kappa}$, definimos a álgebra de Kac-Moody afim estendida $\widetilde{\mathfrak{g}}_{\kappa}$ como

$$
\tilde{\mathfrak{g}}_{\kappa}=\mathfrak{g}((t)) \oplus \mathbb{C} c \oplus \mathbb{C} d,
$$

em que a estrutura de álgebra de Lie de $\widetilde{\mathfrak{g}}_{\kappa}$ é dada pelas relações de comutação

$$
[d, a \otimes f(t)]=a \otimes t \frac{\partial f(t)}{\partial t}, \quad[d, c]=0
$$

para $a \in \mathfrak{g}$ e $f(t) \in \mathbb{C}((t))$. A subálgebra de Cartan correspondente $\widetilde{\mathfrak{h}}$ de $\widetilde{\mathfrak{g}}_{\kappa}$ é definida por

$$
\widetilde{\mathfrak{h}}=\mathfrak{h} \otimes_{\mathbb{C}} \mathbb{C} 1 \oplus \mathbb{C} c \oplus \mathbb{C} d
$$


O espaço dual para $\widetilde{\mathfrak{h}}$ é identificado com $\mathfrak{h}^{*} \oplus \mathbb{C} \Lambda_{0} \oplus \mathbb{C} \delta$, em que $\left.\Lambda_{0}\right|_{\mathfrak{h} \otimes \mathbb{C} 1}=0, \Lambda_{0}(c)=1$, $\Lambda_{0}(d)=0$ e $\left.\delta\right|_{\mathfrak{h} \otimes \mathbb{C} 1}=0, \delta(c)=0, \delta(d)=1$. Logo, o conjunto de raízes de $\widehat{\mathfrak{g}}_{\kappa}$ é naturalmente um subconjunto de $\mathfrak{h}^{*} \oplus \mathbb{C} \Lambda_{0} \oplus \mathbb{C} \delta$ dado por

$$
\widehat{\Delta}=\widehat{\Delta}^{\mathrm{re}} \cup \widehat{\Delta}^{\mathrm{im}}
$$

em que

$$
\widehat{\Delta}^{\mathrm{re}}=\{\alpha+n \delta ; \alpha \in \Delta, n \in \mathbb{Z}\} \quad \text { e } \quad \widehat{\Delta}^{\mathrm{im}}=\{n \delta ; n \in \mathbb{Z} \backslash\{0\}\}
$$

são os conjuntos de raízes reais e imaginárias, respectivamente. Além disso, temos a seguinte decomposição $\widehat{\Delta}=\widehat{\Delta}_{+} \cup \widehat{\Delta}_{-}$em raízes positivas e negativas, em que

$$
\widehat{\Delta}_{+}=\left\{\alpha+n \delta ; \alpha \in \Delta_{+}, n \in \mathbb{Z}_{\geq 0}\right\} \cup\left\{-\alpha+n \delta ; \alpha \in \Delta_{+}, n \in \mathbb{Z}\right\} \cup\{n \delta ; n \in \mathbb{Z}\}
$$

e $\widehat{\Delta}_{-}=-\widehat{\Delta}_{+}$. Também definimos $\widehat{\Delta}_{ \pm}^{\mathrm{re}}=\widehat{\Delta}_{ \pm} \cap \widehat{\Delta}^{\mathrm{re}}$ e $\widehat{\Delta}_{ \pm}^{\mathrm{im}}=\widehat{\Delta}_{ \pm} \cap \widehat{\Delta}^{\mathrm{im}}$. (Mais detalhes podem ser encontrados $[\operatorname{Kac} 90])$.

\section{2 Álgebras de vertex e suas representações}

Nesta seção, recordaremos brevemente as definições, principalmente, para fixar a notação. Para detalhes, nos referimos a [FHL93, Kac98, FB04, LL04, DSK06, Kac17].

Definição 2.2.1 (Definição 3.1.1 em [LL04]). Uma álgebra de vertex consiste em um espaço vetorial $V$ munido de um mapa linear (mapa do operador de vertex) $V \otimes V \rightarrow V\left[\left[z, z^{-1}\right]\right]$, ou equivalente, um mapa linear

$$
\begin{aligned}
Y(\cdot, z): V & \rightarrow(\text { End } V)\left[\left[z, z^{-1}\right]\right], \\
v & \mapsto Y(v, z)=v(z)=\sum_{n \in \mathbb{Z}} v_{n} z^{-n-1} .
\end{aligned}
$$

Chamamos $Y(v, z)$ de o operador de vertex associado ${ }^{1}$ com v. Também temos um elemento distinguido $|0\rangle$ de $V$ (o vetor vácuo). As seguintes condições são assumidas para $u, v \in V$ :

a) A propriedade do vácuo: $Y(|0\rangle, z)=\mathrm{id}_{V}$,

b) A condição de truncamento: $u_{n} v=0$ para $n \gg 0, u, v \in V$, ou seja, $\left.Y(u, z) v \in V((z))\right)$;

\footnotetext{
${ }^{1}$ Observamos que usamos a notação $v_{n}$ para o elemento de End $V$, que é o coeficiente de $z^{-n-1}$ na série formal $Y(v, z)$; a notação $v_{n}$ não se refere a nenhum tipo de componente do vetor $v$.
} 
c) A propriedade da criação ${ }^{2}: Y(v, z)|0\rangle \in V[[z]]$ e $v_{(-1)}|0\rangle=v$;

d) A identidade de Jacobi:

$$
\begin{aligned}
& z_{0}^{-1} \delta\left(\frac{z_{1}-z_{2}}{z_{0}}\right) Y\left(u, z_{1}\right) Y\left(v, z_{2}\right)-z_{0}^{-1} \delta\left(\frac{z_{2}-z_{1}}{-z_{0}}\right) Y\left(v, z_{2}\right) Y\left(u, z_{1}\right) \\
& =z_{2}^{-1} \delta\left(\frac{z_{1}-z_{0}}{z_{2}}\right) Y\left(Y\left(u, z_{0}\right) v, z_{2}\right)
\end{aligned}
$$

Observação 2.2.2. Advertimos que a Definição 2.2.1 e a definição original de álgebras de vertex dada por Borcherds em [Bor86] são equivalentes (Proposição 3.6.6 em [LL04]). Ademais, existem na literatura outras definições equivalentes das álgebras de vertex, veja por exemplo [DSK06].

Observação 2.2.3. Segue-se da propriedade de criação que $Y(v, z)=0$ se, e somente se, $v=0$, de modo que o mapa do operador de vertex $Y(\cdot, z)$ é injetivo. Assim, podemos colocar uma estrutura de álgebra de vertex no espaço vetorial, consistindo em todos os operadores de vertex $Y(v, z)(v \in V)$ (um subespaço de (End $V$ ) [[z, $\left.\left.z^{-1}\right]\right]$ ), para que o mapa $Y(\cdot, z)$ se torne um isomorfismo entre duas álgebras de vertex.

Observação 2.2.4. A partir da identidade de Jacobi, podemos obter as duas fórmulas importantes a seguir. Primeiro, tomando $\operatorname{Res}_{z_{0}}$ da identidade de Jacobi, obtemos a fórmula do comutador:

$$
\left[Y\left(u, z_{1}\right), Y\left(v, z_{2}\right)\right]=\operatorname{Res}_{z_{0}} z_{2}^{-1} \delta\left(\frac{z_{1}-z_{0}}{z_{2}}\right) Y\left(Y\left(u, z_{0}\right) v, z_{2}\right)
$$

Agora, substituindo $\operatorname{Res}_{z_{1}}$ por $\operatorname{Res}_{z_{0}}$ na identidade de Jacobi, obtemos a fórmula iterada

$$
Y\left(Y\left(u, z_{0}\right) v, z_{2}\right)=\operatorname{Res}_{z_{1}}\left(z_{0}^{-1} \delta\left(\frac{z_{1}-z_{2}}{z_{0}}\right) Y\left(u, z_{1}\right) Y\left(v, z_{2}\right)-z_{0}^{-1} \delta\left(\frac{z_{2}-z_{1}}{-z_{0}}\right) Y\left(v, z_{2}\right) Y\left(u, z_{1}\right)\right) .
$$

Por fim, simplificando o primeiro termo no lado direito dessa identidade para que a fórmula do comutador se encaixe na seguinte fórmula associadora:

$$
Y\left(Y\left(u, z_{0}\right) v, z_{2}\right)-Y\left(u, z_{0}+z_{2}\right) Y\left(v, z_{2}\right)=-\operatorname{Res}_{z_{1}} z_{0}^{-1} \delta\left(\frac{z_{2}-z_{1}}{-z_{0}}\right) Y\left(v, z_{2}\right) Y\left(u, z_{1}\right)
$$

Observação 2.2.5. Multiplicando os dois lados da fórmula do comutador pelo polinômio $\left(z_{1}-z_{2}\right)^{k}$, em que $k$ é um número inteiro não negativo suficientemente grande, obtemos a relação fraca de comutação

$$
\left(z_{1}-z_{2}\right)^{k}\left[Y\left(u, z_{1}\right), Y\left(v, z_{2}\right)\right]=0 .
$$

Analogamente, aplicamos a fórmula do associador a um terceiro vetor $w$. Então, multiplicando ambos os lados por $\left(z_{0}+z_{2}\right)^{\ell}$ para um número inteiro não negativo suficientemente grande $\ell$, podemos fazer o lado direito desaparecer, para obter a relação fraca de associatividade

$$
\left(z_{0}+z_{2}\right)^{\ell} Y\left(Y\left(u, z_{0}\right) v, z_{2}\right) w=\left(z_{0}+z_{2}\right)^{\ell} Y\left(u, z_{0}+z_{2}\right) Y\left(v, z_{2}\right) w
$$

Observação 2.2.6. Para uma álgebra de vertex $V$, igualando o coeficiente de $z_{0}^{-\ell-1} z_{1}^{-m-1} z_{2}^{-n-1}$ na

\footnotetext{
${ }^{2} \mathrm{Ou}$ seja, $Y(v, z)|0\rangle$ envolve apenas potências inteiras não negativas de $z$ e o termo constante é $v$.
} 
identidade de Jacobi, obtemos a identidade de Borcherds ${ }^{3}$

$$
\sum_{i=0}^{\infty}\left(\begin{array}{c}
m \\
i
\end{array}\right)\left(u_{\ell+i} v\right)_{m+n-i}=\sum_{i=0}^{\infty}(-1)^{i}\left(\begin{array}{l}
\ell \\
i
\end{array}\right)\left(u_{m+\ell-i} v_{n+i}-(-1)^{\ell} v_{n+\ell-i} u_{m+i}\right)
$$

para todo $\ell, m, n \in \mathbb{Z}, u, v \in V$. Em particular, ao considerar $\ell=0$ na identidade de Borcherds, temos a fórmula do comutador

$$
\left[u_{m}, v_{n}\right]=\sum_{i=0}^{\infty}\left(\begin{array}{c}
m \\
i
\end{array}\right)\left(u_{i} v\right)_{m+n-i}
$$

e definindo $m=0$ na identidade de Borcherds, temos a fórmula iterada

$$
\left(u_{\ell} v\right)_{n}=\sum_{i=0}^{\infty}(-1)^{i}\left(\begin{array}{l}
\ell \\
i
\end{array}\right)\left(u_{\ell-i} v_{n+i}-(-1)^{\ell} v_{n+\ell-i} u_{i}\right)
$$

\subsection{1 Álgebras de operadores de vertex}

Todas as álgebras de vertex que consideramos neste trabalho são $\mathbb{Z}$-graduadas.

Definição 2.2.7 (Definição 1.2.1 em [FZ92]). A álgebra do operador do vertex $\mathcal{V}$ é um quádruplo $(V, Y,|0\rangle, \omega)$, em que $V=\bigoplus_{n \in \mathbb{Z}_{\geq 0}} V_{(n)}$ é um espaço vetorial $\mathbb{Z}_{\geq 0}$-graduado, equipado com uma estrutura de álgebra de vertex $(V, Y,|0\rangle)$ e um vetor homogêneo distinguido $\omega$ (o vetor conformal) de peso $2\left(\omega \in V_{(2)}\right)$, satisfazendo as seguintes condições:

a) As relações da álgebra de Virasoro:

$$
[L(m), L(n)]=(m-n) L(m+n)+\frac{m^{3}-m}{12} \delta_{m+n, 0} \mathbf{c}_{V}
$$

para $m, n \in \mathbb{Z}$, em que

$$
Y(\omega, z)=\sum_{n \in \mathbb{Z}} L(n) z^{-n-2}=\sum_{n \in \mathbb{Z}} \omega_{n} z^{-n-1}
$$

isso é, $L(n):=w_{n+1}$ para $n \in \mathbb{Z}, e \mathbf{c}_{V} \in \mathbb{C}$ é chamado posto ou carga central de $V$.

b) $L(0) v=n v$ para $n \in \mathbb{Z}$ e $v \in V_{(n)}$.

c) $\left(L(-1)\right.$ - propriedade derivada) $: Y(L(-1) v, z)=\frac{d}{d z} Y(v, z)$ para todo $v \in V$.

\footnotetext{
${ }^{3}$ Quando essa identidade é aplicada a qualquer elemento de $\mathrm{V}$, cada soma acima de $i$ se torna finita. Além disso, a soma acima de $i$ no lado direito já é finita.
} 
Definição 2.2.8. Um elemento $v \in V$ é chamado homogêneo e tem peso ou grau n se $v$ estiver em $V_{n}$. Escrevemos $w t(v)=n$.

Observação 2.2.9. Lembramos que, em [FHL93] (veja a Definição 2.2.1), as álgebras do operador de vertex são $\mathbb{Z}$-graduadas de modo que $\operatorname{dim} V_{(n)}<\infty$ para todo $n \in \mathbb{Z}$ e $V_{(n)}=0$ para $n \ll 0$. Ambas as definições são casos particulares de álgebras conformais ${ }^{4}$ (veja a Definição 2.5.8. [FB04]).

\subsubsection{Módulos sobre álgebras de vertex}

Definição 2.2.10 (Definição 4.1.1 em [LL04]). Seja $\mathcal{V}=(V, Y,|0\rangle)$ uma álgebra de vertex. Um $\mathcal{V}$-módulo é um espaço vetorial $M$ equipado com um mapa linear $V \otimes M \rightarrow M\left[\left[x, x^{-1}\right]\right]$, que pode ser expresso de forma equivalente como um mapa linear

$$
\begin{aligned}
Y_{M}(\cdot, z): V & \rightarrow(\text { End } M)\left[\left[z, z^{-1}\right]\right], \\
V & \mapsto Y_{M}(v, z)=v(z)=\sum_{n \in \mathbb{Z}} v_{n} z^{-n-1},
\end{aligned}
$$

de modo que todas as propriedades que definem uma álgebra de vertex que fazem sentido se mantêm. Isto é,

a) A propriedade de vácuo: $Y_{M}(|0\rangle, z)=\mathrm{id}_{M}$,

b) A condição de truncamento: Para cada $v \in V$ e $w \in M$, o endomorfismo $v_{n}$ de $M$ satisfaz $v_{n} m=0$ para $n \gg 0$, ou equivalentemente, $Y_{M}(v, z) w \in M((z))$;

c) A identidade de Jacobi $i^{5}:$ Para cada $u, v \in V$

$$
\begin{aligned}
& z_{0}^{-1} \delta\left(\frac{z_{1}-z_{2}}{z_{0}}\right) Y_{M}\left(u, z_{1}\right) Y_{M}\left(v, z_{2}\right)-z_{0}^{-1} \delta\left(\frac{z_{2}-z_{1}}{-z_{0}}\right) Y_{M}\left(v, z_{2}\right) Y_{M}\left(u, z_{1}\right) \\
& =z_{2}^{-1} \delta\left(\frac{z_{1}-z_{0}}{z_{2}}\right) Y_{M}\left(Y\left(u, z_{0}\right) v, z_{2}\right) .
\end{aligned}
$$

Observação 2.2.11. A álgebra de vertex $\mathcal{V}$ é claramente um $\mathcal{V}$-módulo (uma vez que um módulo é um espaço no qual $V$ age de modo que todas as propriedades definidoras de uma álgebra de vertex fazem sentido!). Como tal, é chamado módulo adjunto, como na teoria das álgebras de Lie.

Observação 2.2.12. Observamos que, ao contrário da Observação 2.2.3, o mapa $Y_{M}(\cdot, z)$ geralmente não é injetivo e, portanto, um módulo $M$ não necessariamente pode ser identificado com um espaço de operadores de vertex $\mathcal{V}$.

\footnotetext{
${ }^{4}$ No caso de álgebras não graduadas, veja a Definição 4.10 em [Kac98].

${ }^{5}$ No lado direito, $Y\left(u, z_{0}\right)$ age no $V$, não no $M$.
} 
Observação 2.2.13. Pode ser usada a seguinte identidade equivalente à identidade de Jacobi da representação definida para álgebras de vertex (veja, por exemplo, a Definição 18 em [Kac17])

$$
\sum_{i=0}^{\infty}\left(\begin{array}{l}
p \\
i
\end{array}\right)\left(u_{(r+i)} v\right)_{(p+q-i)}^{M}=\sum_{i=1}^{\infty}(-1)^{i}\left(\begin{array}{l}
r \\
i
\end{array}\right)\left(u_{(p+r-i)}^{M} v_{(q+i)}^{M}-(-1)^{r} v_{(q+r-i)}^{M} u_{(p+i)}^{M}\right)
$$

em End $M$ para todo $p, q, r \in \mathbb{Z}, u, v \in V$.

\subsubsection{Módulos sobre álgebras de operadores de Vertex}

Agora vamos definir a noção de módulo para uma álgebra de operadores de vertex $\mathcal{V}=$ $(V, Y,|0\rangle, \omega)$.

Definição 2.2.14 (Definição $2 \mathrm{em}$ [DLM97]). Seja $\mathcal{V}$ uma álgebra de operadores de vertex. Um $\mathcal{V}$-módulo fraco $M$ é um $\mathcal{V}$-módulo, em que $\mathcal{V}$ é vista simplesmente como uma álgebra de vertex (esqueçendo o elemento conformal).

Definição 2.2.15 (Definição 1.2.2 em [Zhu96]). Seja $\mathcal{V}$ uma álgebra de operadores de vertex. Um $\mathcal{V}$-módulo forte é um $\mathcal{V}$-módulo fraco $\mathbb{C}$-graduado

$$
M=\bigoplus_{s \in \mathbb{C}} M_{(s)},
$$

em que $M_{(s)}=\{w \in M \mid L(0) w=s w\}$, tal que fixado $s \in \mathbb{C}, M_{(s-n)}=0$ para algum $n \in \mathbb{Z}$ suficientemente grande.

Definição 2.2.16 (Definição 2.1 em [DLM97]). Seja $\mathcal{V}$ uma álgebra de operadores de vertex. Um $\mathcal{V}$-módulo ordinário é um $\mathcal{V}$-módulo forte de modo que $\operatorname{dim} M_{(h)}<\infty$ para algum $h \in \mathbb{C}$.

Observação 2.2.17. As representações ordinárias definidas em [DLM97] são chamadas representações em [LL04] (veja a Definição 4.1.6).

Definição 2.2.18 (Definição 2.3 em [DLM97]). Seja $\mathcal{V}$ uma álgebra de operadores de vertex. Um $\mathcal{V}$-módulo admissível é um $\mathcal{V}$-módulo fraco $M$ munido de uma $\mathbb{Z}_{\geq 0}$-graduação

$$
M=\bigoplus_{n \in \mathbb{Z}_{\geq 0}} M_{(n)}
$$

satisfazendo a seguinte condição: se $m \in \mathbb{Z}, n \in \mathbb{Z}_{\geq 0}$ e $v \in V$ for homogêneo,

$$
v_{m} M_{(n)} \subset M_{(n-m+\mathrm{wt}(v)-1)} .
$$


Observação 2.2.19. As representações admissíveis definidas em [DLM97] são chamadas representações em [Zhu96] (veja a Definição 1.2.3) e são chamadas representações de energia positiva em [DSK06] (veja a Definição 2.21).

Definição 2.2.20. Denotaremos por $\mathcal{E}_{+}(\mathcal{V})$ a categoria de $\mathcal{V}$-módulos de energia positiva.

Proposição 2.2.21 ([Zhu96]). Toda representação forte é admissível.

Observação 2.2.22. Em particular, pela proposição acima, temos que todas as representações ordinárias são admissíveis. Portanto, temos:

$\{$ módulos ordinários $\} \subseteq\{$ módulos fortes $\} \subseteq\{$ módulos admissíveis $\} \subseteq\{$ módulos fracos $\}$.

Definição 2.2.23 (Definição 2.3 em [DLM97]). Um $\mathcal{V}$-módulo admissivel $M$ é chamado simples no caso de 0 e $M$ serem os únicos submódulos $\mathbb{Z}_{\geq 0 \text {-graduados. }}$

Duas classes importantes de álgebras de operadores de vertex são as classes de álgebra de operadores de vertex racionais e álgebras de operadores de vertex regulares, definidas da seguinte forma:

Definição 2.2.24 (Definição 1.2.4 em [Zhu96]). Uma álgebra de operador de vertex é racional se o seguinte for válido:

a) Todo $\mathcal{V}$-módulo admissível é completamente redutível;

b) Cada $\mathcal{V}$-módulo admissivel simples é um $\mathcal{V}$-módulo ordinário;

c) $\mathcal{V}$ possui apenas um número finito de módulos simples distintos.

Outra definição para a álgebra de operadores de vertex racional é:

Definição 2.2.25 (Definição 2.3 em [DLM97]). Uma álgebra de operador de vertex $\mathcal{V}$ é chamada racional se todo $\mathcal{V}$-módulo admissível for uma soma direta de $\mathcal{V}$-módulos admissíveis simples.

Observação 2.2.26.

- As duas definições para álgebras racionais de operadores são equivalentes (veja a Observação 2.4 em [DLM97]). Em outras palavras, $\mathcal{V}$ é racional se todo $\mathcal{V}$-módulo admissível for completamente redutível (veja Definição 5.5.1 em [FB04]).

- Não há diferença entre representações fortes e representações admissíveis para álgebras racionais de operadores de vertex (veja o Lema 1.2.1 em [Zhu96]).

O estudo da redutibilidade completa de um módulo fraco arbitrário para uma álgebra de operadores de vertex racional nos leva à seguinte noção de álgebra regular de operadores de vertex:

Definição 2.2.27 (Definição 3.1 em [DLM97]). Diz-se que uma álgebra de operador de vertex $\mathcal{V}$ é regular se cada $\mathcal{V}$-módulo fraco $M$ é uma soma direta de $\mathcal{V}$-módulos ordinários simples.

Observação 2.2.28. Toda álgebra regular de operadores de vertex é racional (veja Observação 3.2 na [DLM97]).

As definições de submódulos, módulos quocientes, módulos simples podem ser encontradas em [ABD04, Li99]. 


\section{3 Álgebra de Zhu}

Seja $\mathcal{V}=(V, Y,|0\rangle, \omega)$ uma álgebra de operadores de vertex. Seguindo [FZ92, Zhu96], construiremos uma álgebra associativa $\mathrm{Zhu}(V)$ associada a $V$ (chamada álgebra de Zhu) definida como:

$$
\mathrm{Zhu}(V)=\frac{V}{O(V)},
$$

em que $O(V)$ é o subespaço de $V$ gerado pelos vetores

$$
u \circ v:=\operatorname{Res}_{z}\left(Y(u, z) \frac{(1+z)^{w t(u)}}{z^{2}} v\right)=\sum_{i \geq 0}\left(\begin{array}{c}
w t(u) \\
i
\end{array}\right) u_{(i-2)} v
$$

com vetores homogêneos $u, v \in V$.

Definimos um produto $*$ em $\mathrm{Zhu}(V)$ pela fórmula:

$$
u * v:=\operatorname{Res}_{z}\left(Y(u, z) v \frac{(1+z)^{w t(u)}}{z}\right)=\sum_{i \geq 0}\left(\begin{array}{c}
w t(u) \\
i
\end{array}\right) u_{(i-1)} v .
$$

O subespaço $O(V)$ é conhecido por ser um ideal bilateral de $V$ com respeito da multiplicação * (cf. [Zhu96]).

Teorema 2.3.1 ([Zhu96]). O produto $*$ induz uma estrutura de álgebra associativa em $\mathrm{Zhu}(V)$ com identidade $\mathbf{1}+O(V)$. O elemento $\omega+O(V)$ é central em $\mathrm{Zhu}(V)$.

Seja $M$ um $\mathcal{V}$-módulo. O bimódulo de Frenkel-Zhu [FZ92] associado a $M$ é o Zhu( $V)$ bimódulo $Z h u(M)$ definido por

$$
\operatorname{Zhu}(M)=\frac{M}{O(M)},
$$

em que $O(M)$ é o subespaço de $M$ gerado pelos elementos

$$
u \circ m:=\sum_{i \geq 0}\left(\begin{array}{c}
\operatorname{wt}(u) \\
i
\end{array}\right) u_{(i-2)} m
$$

com vetores homogêneos $u \in V$ e $m \in M$. A estrutura do bimódulo de Zhu( $M)$ é dada por

$$
u * m=\sum_{i \geq 0}\left(\begin{array}{c}
\operatorname{wt}(u) \\
i
\end{array}\right) u_{(i-1)} m, \quad m * u=\sum_{i \geq 0}\left(\begin{array}{c}
\operatorname{wt}(u)-1 \\
i
\end{array}\right) u_{(i-1)} m .
$$

Observamos que

$$
u * m-m * u=\sum_{i \geq 0}\left(\begin{array}{c}
\mathrm{wt}(u)-1 \\
i
\end{array}\right) u_{(i)} m
$$


Lema 2.3.2 ([FZ92, Proposição 1.5.4]). O mapa $M \mapsto \mathrm{Zhu}(M)$ define um funtor exato à direita de $\mathcal{V}$-Mod $a \mathrm{Zhu}(\mathcal{V})$-biMod.

Definição 2.3.3. Dada uma representação de energia positiva simples $M=\bigoplus_{n \geq 0} M_{n}$ de $\mathcal{V}$, chamamos $M_{n}$ o n-ésimo nível e $M_{0}:=M_{t o p} \neq 0$ o nível superior de $M$.

Denotamos por $o^{M}(u):=u_{(w t(u)-1)}^{M}$ para todos os elementos homogêneos $u \in V$ e se estende linearmente a $V$. Então $o^{M}(u) M_{n} \subseteq M_{n}$, para que $o(u)$ preserve os componentes homogêneos de qualquer representação graduada de $\mathcal{V}$. Em particular, $o^{M}(u)$ preserva o nível superior.

Lema 2.3.4 (Teorema 1.4.1 em [FZ92]). Para um $\mathcal{V}$-módulo $M$ admissível, quando restrito ao nivel superior $M_{t o p}$, as identidades

$$
o^{M}(u) o^{M}(v)=o^{M}(u * v) \text { e } o^{M}\left(u^{\prime}\right)=0
$$

são preservadas para todo $u, v \in V$ e $u^{\prime} \in O(V)$. Portanto, o nível superior $M_{\text {top }}$ é um Zhu(V)-módulo sob a ação $(u+O(V)) \cdot m=o^{M}(u) m$.

O próximo resultado mostra a importância da álgebra de Zhu na teoria da álgebra de vertex:

Teorema 2.3.5 (Teorema 2.1.2 e Teorema 2.2.2 em [Zhu96]).

a) Para qualquer representação de energia positiva $M$ de $\mathcal{V}$, a representação $\bar{a} \mapsto o(a)$ de $\mathrm{Zhu}(\mathcal{V})$ em $M_{\text {top }}$ está bem definida.

b) A correspondência $M \mapsto M_{\text {top }}$ fornece uma bijeção entre o conjunto de classes de isomorfismo de representações de energia positiva irredutiveis de $\mathcal{V}$ e a de $Z h u(\mathcal{V})$-módulos simples.

A cada álgebra de vertex $\mathbb{Z}$-graduada $\mathcal{V}$, podemos associar uma álgebra de Lie ${ }^{6}$

$$
U(\mathcal{V})=\left(\mathcal{V} \otimes_{\mathbb{C}} \mathbb{C}((t))\right) / \operatorname{Im} \partial
$$

em que

$$
\partial=T \otimes \mathrm{id}+\mathrm{id} \otimes \partial_{t}
$$

Se denotarmos por $a_{[n]}$ por $a \in \mathcal{V}$ e $n \in \mathbb{Z}$ a projeção de $a \otimes t^{n} \in \mathcal{V} \otimes_{\mathbb{C}} \mathbb{C}((t))$ em $U(\mathcal{V})$, então o colchete de Lie em $U(\mathcal{V})$ é dado por

$$
\left[a_{[m]}, b_{[n]}\right]=\sum_{k=0}^{\infty}\left(\begin{array}{c}
m \\
k
\end{array}\right)\left(a_{(k)} b\right)_{[m+n k]}
$$

para $a, b \in \mathcal{V}$ e $m, n \in \mathbb{Z}$.

Também, para um elemento homogêneo $a \in \mathcal{V}$, definimos $\operatorname{deg} a_{[n]}=-n+\operatorname{deg} a-1$. Então, o grau associado aos elementos de $U(\mathcal{V})$ nos dá uma decomposição triangular

$$
U(\mathcal{V})=U(\mathcal{V})_{-} \oplus U(\mathcal{V})_{0} \oplus U(\mathcal{V})_{+}
$$

\footnotetext{
${ }^{6}$ introduzida pela primeira vez por Borcherds [Bor86]
} 
juntamente com um homomorfismo canônico sobrejetor

$$
U(\mathcal{V})_{0} \rightarrow \mathrm{Zhu}(\mathcal{V})
$$

de álgebras de Lie definidas por

$$
a_{[\operatorname{deg} a-1]} \mapsto \pi_{\mathrm{Zhu}}(a)
$$

para um elemento homogêneo $a \in \mathcal{V}$.

Vamos considerar um $\mathcal{V}$-módulo $M$. Então $M$ possui também uma estrutura natural de $U(\mathcal{V})$ módulo, pois temos um homomorfismo canônico

$$
U(\mathcal{V}) \rightarrow \text { End } \mathcal{V}
$$

de álgebras de Lie definidas por

$$
a_{[n]} \mapsto a_{(n)}
$$

para $a \in \mathcal{V}$ e $n \in \mathbb{Z}$. Denotamos por $\Omega_{\mathcal{V}}(M)$ o subespaço vetorial de $M$ que consiste nos vetores de peso mínimo, ou seja, temos

$$
\Omega_{\mathcal{V}}(M)=\left\{v \in M ; U(\mathcal{V})_{-} v=0\right\}
$$

Segue-se imediatamente usando a decomposição triangular de $U(\mathcal{V})$ que $\Omega_{\mathcal{V}}(M)$ é um $U(\mathcal{V})_{0}$-módulo. Ademais, por [DLM98], temos que $\Omega_{\mathcal{V}}(M)$ é um Zhu(V)-módulo, em que a ação de $\pi_{\text {Zhu }}(a) \in \operatorname{Zhu}(\mathcal{V})$ para $a \in \mathcal{V}$ é dado por $o_{M}(a)$.

Observamos que, se $M$ é um $\mathcal{V}$-módulo de energia positiva, então $\Omega_{\mathcal{V}}(M) \supset M_{\text {top }}$ e $\Omega_{\mathcal{V}}(M)=$ $M_{\text {top }}$ sempre que $M$ seja um $\mathcal{V}$-módulo simples.

Portanto, $\Omega_{\mathcal{V}}: \mathcal{E}_{+}(\mathcal{V}) \rightarrow \mathcal{M}(\mathrm{Zhu}(\mathcal{V}))$ define um funtor. Por outro lado, existe também um funtor de indução

$$
\mathbb{M}_{\mathcal{V}}: \mathcal{M}(\mathrm{Zhu}(\mathcal{V})) \rightarrow \mathcal{E}_{+}(\mathcal{V})
$$

que é um funtor adjunto à esquerda para $\Omega \mathcal{V}$ e possui a seguinte propriedade universal. Para um $\mathcal{V}$-módulo $M$ e um morfismo $\varphi: E \rightarrow \Omega \mathcal{V}(M)$ de $\operatorname{Zhu}(\mathcal{V})$-módulos, existe um único morfismo $\widetilde{\varphi}: \mathbb{M}_{\mathcal{V}}(E) \rightarrow M$ de $\mathcal{V}$-módulos que estende $\varphi$, veja [DLM98]. Para um Zhu(V)-módulo $E$, temos $\mathbb{M}_{\mathcal{V}}(E)_{\text {top }} \simeq E$ como módulos sobre $\mathrm{Zhu}(\mathcal{V})$. Além disso, como o $\mathcal{V}$-módulo $\mathbb{M}_{\mathcal{V}}(E)$ tem um único $\mathcal{V}$-submódulo maximal $\mathbb{K}_{\mathcal{V}}(E)$ tendo intersecção nula com o Zhu(V)-submódulo $E$ de $\mathbb{M}_{\mathcal{V}}(E)$, podemos definir

$$
\mathbb{L}_{\mathcal{V}}(E)=\mathbb{M}_{\mathcal{V}}(E) / \mathbb{K}_{\mathcal{V}}(E)
$$

para um Zhu(V)-módulo $E$. 


\subsection{1 Álgebras de Vertex afins}

Seja $\mathfrak{g}$ uma álgebra de Lie semissimples (redutiva) de dimensão finita e seja $\kappa$ uma forma bilinear simétrica g-invariante em $\mathfrak{g}$. O $\widehat{\mathfrak{g}}_{\kappa}$-módulo induzido $\mathbb{M}_{\kappa, \mathfrak{g}}(\mathbb{C})=U\left(\widehat{\mathfrak{g}}_{\kappa}\right) \otimes_{U(g[t] \oplus \mathbb{C} c)} \mathbb{C}$, em que $\mathbb{C}$ é o $\mathfrak{g}$-módulo trivial 1-dimensional, tem uma importância especial na teoria das álgebras de vertex, pois está equipado com a estrutura natural de uma álgebra de vertex $\mathbb{Z}_{\geq 0}$-graduada, chamada álgebra de vertex afim universal, veja [Kac98, Kac17], que iremos denotar por $\mathcal{V}_{\kappa}(\mathfrak{g})$. Para um elemento $a \in \mathfrak{g}$, denotamos por $a(z) \in \widehat{\mathfrak{g}}_{\kappa}\left[\left[z^{ \pm 1}\right]\right]$ a distribuição formal definida por

$$
a(z)=\sum_{n \in \mathbb{Z}} a_{n} z^{-n-1}
$$

Usando essa série de potências formais, podemos reescrever as relações de comutação (2.2) para $\widehat{\mathfrak{g}}_{\kappa}$ da forma

$$
[a(z), b(w)]=[a, b](w) \delta(z-w)+\kappa(a, b) c \partial_{w} \delta(z-w)
$$

para $a, b \in \mathfrak{g}$. A correspondência estado campo $Y: \mathcal{V}_{\kappa}(\mathfrak{g}) \rightarrow \operatorname{End} \mathcal{V}_{\kappa}(\mathfrak{g})\left[\left[z^{ \pm 1}\right]\right]$ é dada por

$$
Y\left(a_{1,-n_{1}-1} \ldots a_{k,-n_{k}-1}|0\rangle, z\right)=\frac{1}{n_{1} ! \ldots n_{k} !}: \partial_{z}^{n_{1}} a_{1}(z) \ldots \partial_{z}^{n_{k}} a_{k}(z):
$$

para $k \in \mathbb{N}, n_{1}, n_{2}, \ldots, n_{k} \in \mathbb{Z}_{\geq 0}$ e $a_{1}, a_{2}, \ldots, a_{k} \in \mathfrak{g}$, em que $|0\rangle \in \mathcal{V}_{\kappa}(\mathfrak{g})$ é o vetor de vácuo (um vetor de peso máximo de $\left.\mathbb{M}_{\kappa, \mathfrak{g}}(\mathbb{C})\right)$. O operador de translação $T: \mathcal{V}_{\kappa}(\mathfrak{g}) \rightarrow \mathcal{V}_{\kappa}(\mathfrak{g})$ é definido por $T|0\rangle=0$ e $\left[T, a_{n}\right]=-n a_{n-1}$ por $a \in \mathfrak{g}$ e $n \in \mathbb{Z}$.

Para descrever representações de energia positiva de $\mathcal{V}_{\kappa}(\mathfrak{g})$, precisamos conhecer a álgebra de Zhu. Dado que para $\mathcal{V}_{\kappa}(\mathfrak{g})$, temos um isomorfismo canônico [FZ92]

$$
\operatorname{Zhu}\left(\mathcal{V}_{\kappa}(\mathfrak{g})\right) \simeq U(\mathfrak{g})
$$

de álgebras associativas determinado por

$$
a_{1,-n_{1}-1} a_{2,-n_{2}-1} \ldots a_{k,-n_{k}-1}|0\rangle \mapsto(-1)^{n_{1}+n_{2}+\cdots+n_{k}} a_{k} \ldots a_{2} a_{1}
$$

para $k \in \mathbb{N}, n_{1}, n_{2}, \ldots, n_{k} \in \mathbb{Z}_{\geq 0}$ e $a_{1}, a_{2}, \ldots, a_{k} \in \mathfrak{g}$.

Observamos que de acordo com o Teorema 2.3.5 aplicado a álgebra de vertex afim universal, o mapa $E \mapsto \mathbb{L}_{\kappa, \mathfrak{g}}(E)$ fornece uma correspondência um a um entre as classes de isomorfismo de $\mathfrak{g}$ módulos simples e $\mathcal{V}_{\kappa}(\mathfrak{g})$-módulos simples de energia positiva. Portanto, o estudo dos $\mathcal{V}_{\kappa}(\mathfrak{g})$-módulos de energia positiva reduz-se ao estudo dos g-módulos.

Ademais, o único quociente simples $\mathbb{L}_{\kappa, \mathfrak{g}}(\mathbb{C})$ de $\mathbb{M}_{\kappa, \mathfrak{g}}(\mathbb{C})$ também possui uma estrutura natural de álgebra de vertex $\mathbb{Z}_{\geq 0}$-graduada, denominada álgebra de vertex afim simples, que iremos denotar por $\mathcal{V}_{\kappa}(\mathfrak{g})$. A álgebra $\operatorname{Zhu}\left(\mathcal{V}_{\kappa}(\mathfrak{g})\right)$ é imagem homomórfica de $U(\mathfrak{g})$, portanto, temos [Ara16]

$$
\operatorname{Zhu}\left(\mathcal{V}_{\kappa}(\mathfrak{g})\right) \simeq U(\mathfrak{g}) / I_{\kappa}
$$


para algum ideal bilateral $I_{\kappa}$ de $U(\mathfrak{g})$. Além do mais, o mapa $E \mapsto \mathbb{L}_{\kappa, \mathfrak{g}}(E)$ fornece uma correspondência um a um entre classes de isomorfismo de módulos simples de $U(\mathfrak{g}) / I_{\kappa}$ e $\mathcal{V}_{\kappa}(\mathfrak{g})$-módulos simples de energia positiva.

\subsection{Representações de energia positiva de $\mathcal{V}_{\kappa}(\mathfrak{g})$}

Nesta seção, descreveremos famílias de representações de energia positiva da álgebra de vertex simples afim $\mathcal{V}_{\kappa}(\mathfrak{g})$ de nível admissível $\kappa$ associado a uma álgebra de Lie simples $\mathfrak{g}$.

\subsubsection{Representações admissíveis}

Seja $\mathfrak{g}$ uma álgebra de Lie simples e complexa e seja $\kappa$ uma forma bilinear simétrica $\mathfrak{g}$-invariante em $\mathfrak{g}$. Como $\mathfrak{g}$ é uma álgebra de Lie simples, temos $\kappa=k \kappa_{0} \operatorname{com} k \in \mathbb{C}$, em que $\kappa_{0}$ é a forma bilinear $\mathfrak{g}$-invariante normalizada em $\mathfrak{g}$ satisfazendo

$$
\kappa_{\mathfrak{g}}=2 h^{\vee} \kappa_{0}
$$

Seja $\widehat{\mathfrak{g}}_{\kappa}$ a álgebra Kac-Moody associada à álgebra de Lie $\mathfrak{g}$ do nível $\kappa$. Para $\lambda \in \widehat{\mathfrak{h}}^{*}$, definimos seu sistema de raízes integral $\widehat{\Delta}(\lambda)$ por

$$
\widehat{\Delta}(\lambda)=\left\{\alpha \in \widehat{\Delta}^{\mathrm{re}} ;\left\langle\lambda+\widehat{\rho}, \alpha^{\vee}\right\rangle \in \mathbb{Z}\right\},
$$

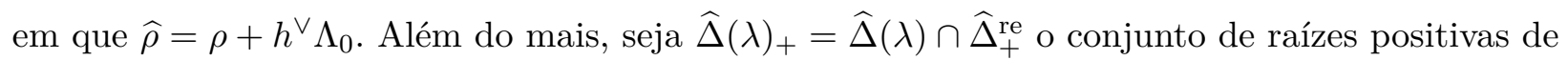
$\widehat{\Delta}(\lambda)$ e $\widehat{\Pi}(\lambda) \subset \widehat{\Delta}(\lambda)_{+}$o conjunto de raízes simples.

Definição 2.4.1 (Definição $1.1 \mathrm{em}$ [KW08]). Um peso $\lambda \in \widehat{\mathfrak{h}}^{*}$ é admissível se satisfaz as seguintes condições:

a) $\lambda$ é dominante regular, ou seja, $\left\langle\lambda+\widehat{\rho}, \alpha^{\vee}\right\rangle \notin \mathbb{Z}_{\leq 0}$ para todo $\alpha \in \widehat{\Delta}_{+}^{r e}$;

b) o $\mathbb{Q}$-span de $\widehat{\Delta}(\lambda)$ contém $\widehat{\Delta}^{\text {re }}$.

Em particular, se $\lambda=k \Lambda_{0}$ é um peso admissível para $k \in \mathbb{C}$, então $k$ é chamado de número admissível. 
Os números admissíveis para cada álgebra de Lie simples $\mathfrak{g}$ foram descritos em [KW89, KW08] do seguinte modo.

Proposição 2.4.2 (Proposição 1.2 em [KW08]). O número $k$ é admissível se, e somente se,

$$
k+h^{\vee}=\frac{p}{q} \quad \text { comp } p \in \mathbb{N},(p, q)=1, p \geq\left\{\begin{array}{ll}
h^{\vee} & \text { se }\left(r^{\vee}, q\right)=1, \\
h & \text { se }\left(r^{\vee}, q\right)=r^{\vee}
\end{array},\right.
$$

em que $r^{\vee}$ é o número de laços de $\mathfrak{g}$, i.e., o número máximo de arestas no diagrama de Dyinkin da álgebra de Lie $\mathfrak{g}$.

Como a admissibilidade de um número $k \in \mathbb{C}$ depende apenas de $\mathfrak{g}$, diremos que $k$ é um número admissível para $\mathfrak{g}$. Além disso, vamos supor que $k \in \mathbb{Q}$ seja um número admissível para $\mathfrak{g}$.

Definição 2.4.3 ([AFR17]). Dizemos que um $\mathfrak{g}$-módulo E é admissível de nível $k$ se $\mathbb{L}_{k \kappa_{0}, \mathfrak{g}}(E)$ é um $\mathcal{L}_{k \kappa_{0}}(\mathfrak{g})$-módulo, ou equivalentemente se E é um $\operatorname{Zhu}\left(\mathcal{L}_{k \kappa_{0}}(\mathfrak{g})\right)$-módulo.

Em particular, o ga-módulo de peso máximo simples $L_{\mathfrak{b}}^{\mathfrak{g}}(0)$ com peso máximo zero é um $\mathfrak{g}$-módulo admissível de nível $k$. Além disso, dado que $\mathrm{Zhu}\left(\mathcal{L}_{k \kappa_{0}}(\mathfrak{g})\right) \simeq U(\mathfrak{g}) / I_{k}$, em que $I_{k}$ é um ideal bilateral de $U(\mathfrak{g})$, obtemos que um $\mathfrak{g}$-módulo $E$ é admissível de nível $k$ se, e somente se, o ideal $I_{k}$ estiver contido no anulador $\operatorname{Ann}_{U(\mathfrak{g})} E$.

Os g-módulos de peso máximo admissíveis de nível $k$ foram classificados em [Ara16] da seguinte forma. Seja $\operatorname{Pr}_{k}$ o conjunto de pesos admissíveis $\lambda \in \widehat{\mathfrak{h}}^{*}$ de nível $k$ de modo que exista um elemento $y \in \widetilde{W}$ do grupo de Weyl afim extendido $\widetilde{W}$ de $\mathfrak{g}$ satisfazendo $\widehat{\Delta}(\lambda)=y\left(\widehat{\Delta}\left(k \Lambda_{0}\right)\right)$. Ademais, vamos definir o subconjunto

$$
\overline{\operatorname{Pr}}_{k}:=\left\{\bar{\lambda}: \lambda \in \operatorname{Pr}_{k}\right\}
$$

de $\mathfrak{h}^{*}$, em que $\bar{\lambda} \in \mathfrak{h}^{*}$ indica a projeção canônica de $\lambda \in \widehat{\mathfrak{h}}^{*}$ a $\mathfrak{h}^{*}$.

Teorema 2.4.4 (Teorema $9.1 \mathrm{em}[\mathrm{Ara15b}]$ ). Seja $k \in \mathbb{Q}$ um número admissivel para $\mathfrak{g}$. Então o $\mathfrak{g}$-módulo simples de peso máximo $L_{\mathfrak{b}}^{\mathfrak{g}}(\lambda)$ com peso máximo $\lambda \in \mathfrak{h}^{*}$ é admissível de nível $k$ se, $e$ somente se, $\lambda \in \overline{\operatorname{Pr}}_{k}$.

Denotando o anulador de $L_{\mathfrak{b}}^{\mathfrak{g}}(\lambda)$ em $U(\mathfrak{g})$ por $J_{\lambda}$.

Lembramos que o Teorema de Duflo [Duf77] afirma que, para qualquer ideal primitivo $I$ de $U(\mathfrak{g})$, existe $\lambda \in \mathfrak{h}^{*}$ tal que $I=J_{\lambda}$. Isso implica que

Corolário 2.4.5 (Corolário 9.2 em [Ara15b]). Um g-módulo de peso simples $E$ é admissível de nível $k$ se, e somente se, $\operatorname{Ann}_{U(\mathfrak{g})} E=J_{\lambda}$ para algum $\lambda \in \overline{\operatorname{Pr}}_{k}$.

Ainda, para $\lambda, \mu \in \overline{\operatorname{Pr}}_{k}$, temos $J_{\lambda}=J_{\mu}$ se, e somente se, existe $w \in W$ de forma que $\mu=w \cdot \lambda$ (veja Corolário 2.3 em [FHR20]). Portanto, podemos definir uma relação de equivalência em $\overline{\operatorname{Pr}}_{k}$ por

$$
\lambda \sim \mu \Longleftrightarrow \text { existe } w \in W \text { tal que } \mu=w \cdot \lambda .
$$

Consequentemente definimos

$$
\left[\overline{\operatorname{Pr}}_{k}\right]=\overline{\operatorname{Pr}}_{k} / \sim
$$


para um número admissível $k$ de $\mathfrak{g}$.

Definição 2.4.6 ([FK20]). Seja $k \in \mathbb{Q}$ um número admissível para $\mathfrak{g}$. Denotamos por $\Omega_{k}(\mathfrak{p})$ o conjunto de pesos $\lambda \in \overline{\operatorname{Pr}}_{k} \cap \Lambda^{+}(\mathfrak{p})$.

Observação 2.4.7. Se $\lambda \in \Omega_{k}(\mathfrak{p})$, então $\left\langle\lambda+\rho, \alpha^{\vee}\right\rangle \in \mathbb{Z}_{>0}$, para todo $\alpha \in \Delta_{+}^{\mathfrak{l}}$, e $\left\langle\lambda+\rho, \alpha^{\vee}\right\rangle \notin \mathbb{Z}$, para todo $\alpha \in \Delta_{+}^{\mathfrak{u}}$.

Corolário 2.4.8 ([FK20]). Suponhamos $\lambda \in \Omega_{k}(\mathfrak{p})$. O módulo de Verma generalizado $M_{\mathfrak{p}}^{\mathfrak{g}}(\lambda)$ é um $\mathfrak{g}$-módulo simples se, e somente se, $\left\langle\lambda+\rho, \alpha^{\vee}\right\rangle \notin \mathbb{Z}_{>0}$ para todo $\alpha \in \Delta_{+}^{\mathfrak{u}}$.

Teorema 2.4.9 (Teorema 6.2 em [FK20]). Seja $\mathfrak{p}$ uma subálgebra parabólica padrão de $\mathfrak{g}$ e seja $k \in \mathbb{Q}$ um número admissível para $\mathfrak{g}$. Então, o $\mathfrak{g}$-módulo $W_{\mathfrak{p}}^{\mathfrak{g}}(\lambda, \alpha)$ é admissível de nível $k$ para $\lambda \in \Omega_{k}(\mathfrak{p})$ e $\alpha \in \Delta_{+}^{\mathfrak{u}}$.

\subsection{2 Órbitas de Richardson e variedades associadas}

Seja $G$ um grupo algébrico semissimples complexo conexo com sua álgebra de Lie g. Denotamos por $\mathcal{N}(\mathfrak{g})$ o cone nilpotente de $\mathfrak{g}$, ou seja, o conjunto de elementos nilpotentes de $\mathfrak{g} . \mathcal{N}(\mathfrak{g})$ é uma subvariedade algébrica fechada irredutível de $\mathfrak{g}$ e uma união finita de $G$-órbitas. Existe uma única órbita nilpotente de $\mathfrak{g}$, denotada por $\mathcal{O}_{\text {reg }}$ e chamada órbita nilpotente regular ou principal de $\mathfrak{g}$, que é um subconjunto aberto denso de $\mathcal{N}(\mathfrak{g})$. Então, como $\mathfrak{g}$ é simples, existe uma única órbita nilpotente de $\mathfrak{g}$ que é um subconjunto denso e aberto de $\mathcal{N}(\mathfrak{g}) \backslash \mathcal{O}_{\text {reg }}$, simbolizado por $\mathcal{O}_{\text {subreg }}$ e denominado de órbita subregular nilpotente de $\mathfrak{g}$. Além disso, existe uma órbita nilpotente de $\mathfrak{g}$ não nula de dimensão mínima, indicada por $\mathcal{O}_{\text {min }}$ e chamada de órbita minimal nilpotente de $\mathfrak{g}$, a qual está contida no fecho de todas as órbitas nilpotentes não nulas de $\mathfrak{g}$. Finalmente $\mathcal{O}_{\text {zero, }}$, denotamos a órbita nilpotente nula de $\mathfrak{g}$. A dimensão de cada uma destas órbitas nilpotentes de $\mathfrak{g}$ é apresentada na Figura 2.1. Para obter detalhes sobre órbitas nilpotentes em álgebras de Lie semissimples, veja [CM93].

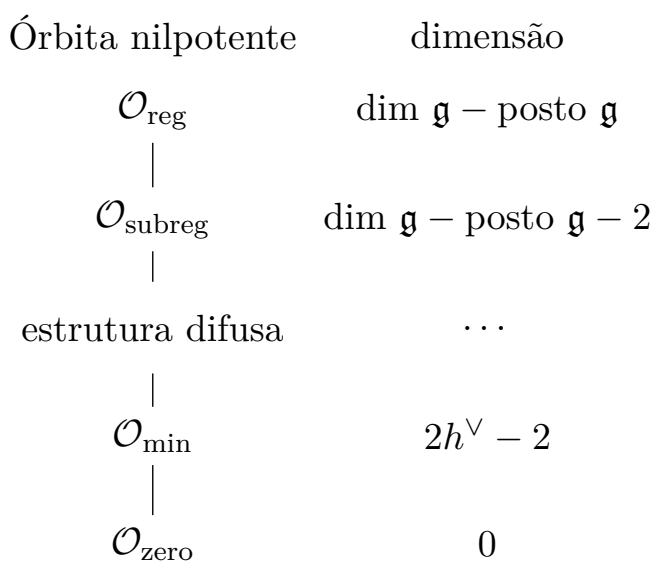

Figura 2.1: Diagrama de Hasse de órbitas nilpotentes 
Exemplo 2.4.10. Seja $\mathfrak{g}=\mathfrak{s l}_{6}$. Apresentamos na Figura 2.2 a descrição do poset para $\mathfrak{s l}_{6}$. A coluna à direita indica a dimensão das órbitas que aparecem na mesma linha.

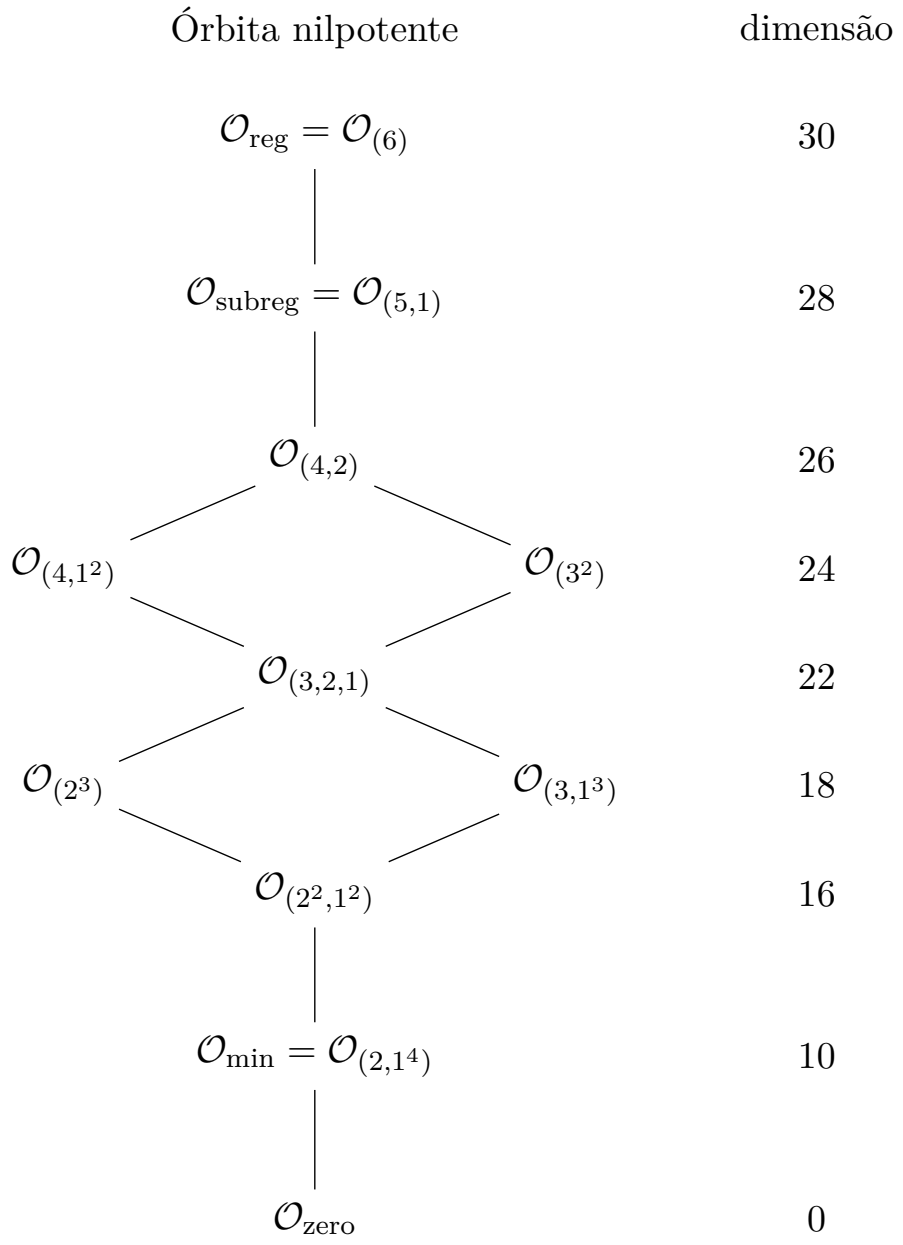

Figura 2.2: Diagrama de Hasse de órbitas nilpotentes

Vamos considerar a filtração PBW em $U(\mathfrak{g})$ e a álgebra graduada associada gr $U(\mathfrak{g}) \simeq S(\mathfrak{g}) \simeq$ $\mathbb{C}\left[\mathfrak{g}^{*}\right]$. A variedade associada $\mathcal{V}(I)$ de um ideal à esquerda $I$ de $U(\mathfrak{g})$ é definida como o zero locus em $\mathfrak{g}^{*}$ do ideal graduado gr $I$ de $S(\mathfrak{g})$. Além disso, se $I$ é um ideal bilateral de $U(\mathfrak{g})$, então $I$ e gr $I$ são invariantes sob a ação adjunta de $G$. Consequentemente, a variedade associada é uma união de $G$-órbitas de $\mathfrak{g}^{*}$. Desse modo, temos

$$
\mathcal{V}(I)=\operatorname{Specm}(S(\mathfrak{g}) / \operatorname{gr} I)=\operatorname{Specm}(S(\mathfrak{g}) / \sqrt{\operatorname{gr} I}),
$$

em que $\sqrt{\operatorname{gr} I}$ denota o radical de gr $I$. Como a forma de Cartan-Killing $\kappa_{\mathfrak{g}}$ é uma forma bilinear simétrica invariante em $\mathfrak{g}$, ela fornece uma correspondência um a um entre órbitas adjuntas de $\mathfrak{g}$ e órbitas coadjuntas de $\mathfrak{g}^{*}$. Para uma órbita adjunta $\mathcal{O}$ de $\mathfrak{g}$, denotamos por $\mathcal{O}^{*}$ a órbita coadjunta correspondente de $\mathfrak{g}^{*}$. Para um ideal primitivo $I$ de $U(\mathfrak{g})$, a variedade associada $\mathcal{V}(I)$ é o fecho de $\mathcal{O}^{*}$ para alguma órbita nilpotente $\mathcal{O}$ de $\mathfrak{g}$, veja [Jos85].

Definição 2.4.11 ([AFR17]). Se E é um $\mathfrak{g}$-módulo simples e $\mathcal{V}\left(A n n_{U(\mathfrak{g})} E\right)=\overline{\mathcal{O}^{*}}$ para uma órbita nilpotente $\mathcal{O}$ de $\mathfrak{g}$, dizemos que $E$ pertence a $\mathcal{O}$.

Para uma descrição das órbitas nilpotentes dos g-módulos admissíveis, nos referimos a [Ara15a]. 
Teorema 2.4.12 (Teorema $9.3 \mathrm{em}[$ Ara15b]). Seja $k \in \mathbb{Q}$ um número admissível para $\mathfrak{g}$ com o denominador $q \in \mathbb{Z}_{>0}$. Existe uma órbita nilpotente $\mathcal{O}_{q}$ de $\mathfrak{g}$ tal que

$$
\mathcal{V}\left(I_{k}\right)=\overline{\mathcal{O}_{q}^{*}}
$$

e temos

$$
\overline{\mathcal{O}_{q}}= \begin{cases}\left\{x \in \mathfrak{g} ;(\operatorname{ad} x)^{2 q}=0\right\}, & \text { se }\left(r^{\vee}, q\right)=1, \\ \left\{x \in \mathfrak{g} ; \pi_{\theta_{s}}(x)^{2 q / r^{\vee}}=0\right\}, & \text { se }\left(r^{\vee}, q\right)=r^{\vee},\end{cases}
$$

em que $\pi_{\theta_{s}}: \mathfrak{g} \rightarrow$ End $L_{\mathfrak{b}}^{\mathfrak{g}}\left(\theta_{s}\right)$ é o $\mathfrak{g}$-módulo simples de peso máximo $\theta_{s}$.

Seja $\mathcal{O}$ uma órbita nilpotente de $\mathfrak{g}$ e seja $k \in \mathbb{Q}$ um número admissível para $\mathfrak{g}$ com o denominador $q \in \mathbb{Z}_{>0}$. Definimos o subconjunto

$$
\overline{\operatorname{Pr}}_{k}^{\mathcal{O}}=\left\{\lambda \in \overline{\operatorname{Pr}}_{k} ; \mathcal{V}\left(J_{\lambda}\right)=\overline{\mathcal{O}^{*}}\right\}
$$

de $\mathfrak{h}^{*}$. Então, um $\mathfrak{g}$-módulo simples $E$ na órbita nilpotente $\mathcal{O}$ é admissível de nível $k$ se, e somente se, $\operatorname{Ann}_{U(\mathfrak{g})} E=J_{\lambda}$ para algum $\lambda \in \overline{\operatorname{Pr}}_{k}^{\mathcal{O}}$. Como $I_{k} \subset J_{\lambda}$ para $\lambda \in \overline{\operatorname{Pr}}_{k}$, temos $\mathcal{V}\left(J_{\lambda}\right) \subset \mathcal{V}\left(I_{k}\right)=\overline{\mathcal{O}_{q}^{*}}$ pelo Teorema 2.4.12, o que nos fornece

$$
\overline{\operatorname{Pr}}_{k}=\bigsqcup_{\mathcal{O} \subset \overline{\mathcal{O}}_{q}} \overline{\operatorname{Pr}}_{k}^{\mathcal{O}}
$$

Desse modo, precisamos descrever o subconjunto $\overline{\operatorname{Pr}}_{k}^{\mathcal{O}}$ de $\overline{\operatorname{Pr}}_{k}$ para uma órbita nilpotente $\mathcal{O}$ de $\mathfrak{g}$.

Para $x \in \mathfrak{g}$, denotamos por $\mathfrak{g}^{x}$ o centralizador de $x$ em $\mathfrak{g}$, e para um subconjunto $X$ de $\mathfrak{g}$, definimos o conjunto

$$
X^{\mathrm{reg}}=\left\{x \in X: \operatorname{dim} \mathfrak{g}^{x}=\min _{y \in X} \operatorname{dim} \mathfrak{g}^{y}\right\}
$$

e denominamos de conjunto de elementos regulares em $X$.

Teorema 2.4.13 ([BB82]). Seja $\mathfrak{p}$ uma subálgebra parabólica padrão de $\mathfrak{g}$. Para $\lambda \in \Lambda^{+}(\mathfrak{p})$ a variedade associada $\mathcal{V}\left(A n n_{U(\mathfrak{g})} M_{\mathfrak{p}}^{\mathfrak{g}}(\lambda)\right)$ é o fecho de $\mathcal{O}_{\mathfrak{p}}^{*}$, em que

$$
\mathcal{O}_{\mathfrak{p}}=\left(G \cdot \mathfrak{p}^{\perp}\right)^{\mathrm{reg}}
$$

$e \mathfrak{p}^{\perp}$ é o complemento ortogonal de $\mathfrak{p}$ com relação à forma Cartan-Killing. Em particular, temos

$$
\overline{\mathcal{O}_{\mathfrak{p}}}=G \cdot \mathfrak{p}^{\perp}
$$

e a variedade associada $\mathcal{V}\left(A n n_{U(\mathfrak{g})} M_{\mathfrak{p}}^{\mathfrak{g}}(\lambda)\right)$ é irredutível. A órbita $\mathcal{O}_{\mathfrak{p}}$ é a órbita de Richardson determinada por $\mathfrak{p}$.

Agora, encontramos as subálgebras parabólicas padrão para as quais as órbitas de Richardson correspondentes são as órbitas nilpotentes distintas $\mathcal{O}_{\text {zero }}, \mathcal{O}_{\text {min }}, \mathcal{O}_{\text {subreg }}$ e $\mathcal{O}_{\text {reg. Lembramos que, }}$ enquanto a órbita regular $\mathcal{O}_{\text {reg }}$ e a órbita subregular $\mathcal{O}_{\text {subreg são órbitas de Richardson para qualquer }}$ $\mathfrak{g}$, a órbita minimal $\mathcal{O}_{\text {min }}$ é uma órbita de Richardson apenas para $\mathfrak{s l}_{n+1}, n \geq 1$. Como todas essas 
órbitas nilpotentes são determinadas exclusivamente por suas dimensões e como $\operatorname{dim} \mathcal{O}_{\mathfrak{p}}=2 \operatorname{dim} \mathfrak{u}$ para uma subálgebra parabólica $\mathfrak{p}=\mathfrak{l} \oplus \mathfrak{u}$ de $\mathfrak{g}$ com o nilradical $\mathfrak{u}$, comparando as dimensões obtemos que

a) $\mathcal{O}_{\text {zero }}=\mathcal{O}_{\mathfrak{g}}$

b) $\mathcal{O}_{\min }=\mathcal{O}_{\mathfrak{p}_{\alpha}^{\max }}$ para $\alpha \in\left\{\alpha_{1}, \alpha_{n-1}\right\}$ e $\mathfrak{g}=\mathfrak{s l}_{n+1}, n \geq 1$,

c) $\mathcal{O}_{\text {subreg }}=\mathcal{O}_{\mathfrak{p}_{\alpha}^{\min }}$ para $\alpha \in \Pi$,

d) $\mathcal{O}_{\text {reg }}=\mathcal{O}_{\mathfrak{b}}$,

em que para $\alpha \in \Pi, \mathfrak{p}_{\alpha}^{\max }$ denota a subálgebra parabólica de $\mathfrak{g}$ associada ao subconjunto $\Sigma=\Pi \backslash\{\alpha\}$ e $\mathfrak{p}_{\alpha}^{\min }$ denota a subálgebra parabólica de $\mathfrak{g}$ associada ao subconjunto $\Sigma=\{\alpha\}$.

Proposição 2.4.14 (Proposição $6.6 \mathrm{em}[\mathrm{FK} 20]$ ). Seja $k \in \mathbb{Q}$ um número admissível para $\mathfrak{g}$. Então temos $\Omega_{k}(\mathfrak{b})=\overline{\operatorname{Pr}}_{k}{ }^{\text {reg }}$.

Com base no Teorema 1.3, [KW08], obtemos que o conjunto $\overline{\operatorname{Pr}}_{k}^{\mathcal{O}_{\text {reg }}}$ é não vazio se, e somente se, $\mathcal{V}\left(J_{\lambda}\right)=\overline{\mathbb{O}_{\text {prin }}}=\mathcal{N}$ ou equivalente se, e somente se, $\mathcal{O}_{\text {reg }}=\mathcal{O}_{q}$, em que $q$ é o denominador de $k$. Pelo Teorema 2.4.12 isso vale se, e somente se,

$$
q \geq \begin{cases}h & \text { se }\left(r^{\vee}, q\right)=1, \\ r^{\vee L} h^{\vee} & \text { se }\left(r^{\vee}, q\right)=r^{\vee},\end{cases}
$$

em que $h$ é o número de Coxeter de $\mathfrak{g} \mathrm{e}^{L} h^{\vee}$ é o número dual de Coxeter da álgebra de Lie dual de Langlands ${ }^{L} \mathfrak{g}$ de $\mathfrak{g}$ (veja [AFR17]).

Proposição 2.4.15 (Proposição 1.5 em [KW08]). Seja $k$ um número admissível de $\mathfrak{g}, q \in \mathbb{Z}_{>0}$ o denominador de $k$. Suponhamos que $\left(r^{\vee}, q\right)=1$ e $q \geq h$. Temos uma bijeção

$$
\begin{array}{ccc}
\left(\widehat{P}_{+}^{p-h^{\vee}} \times \widehat{P}_{+}^{\vee}, q-h\right) / \widetilde{W}_{+} & \stackrel{\sim}{\rightarrow} & {\left[\overline{P r}_{k}^{\mathbb{O}_{p r i n}}\right],} \\
{[(\lambda, \mu)]} & \mapsto & {\left[\bar{\lambda}-\frac{p}{q}\left(\bar{\mu}+\rho^{\vee}\right)\right],}
\end{array}
$$

em que $\widetilde{W}_{+}$age diagonalmente em $\widehat{P}_{+}^{p-h^{\vee}} \times{ }^{L} \widehat{P}_{+}^{q-h}, \widehat{P}_{+}^{m}$ é o conjunto de pesos integrais dominantes de nível $m$ de $\widehat{\mathfrak{g}} e^{L} \widehat{P}_{+}^{\vee, m}$ é o conjunto copesos integrais dominantes de nível $m$ da álgebra de Kac-Moody afim $\widehat{L_{\mathfrak{g}}}$ associada à álgebra de Lie dual de Langlands ${ }^{L} \mathfrak{g}$.

\subsubsection{Representações admissíveis de $\mathfrak{s l}_{n+1}$}

Nesta subseção, consideraremos a álgebra de Lie simples $\mathfrak{g}=\mathfrak{s l}_{n+1}$ para $n \geq 1$. Observamos que todas as órbitas nilpotentes de $\mathfrak{g}$ são órbitas de Richardson. 
Proposição 2.4.16 (Proposição 3.1.7 e Proposição 5.4.1 em [CM93]). As órbitas nilpotentes em $\mathfrak{g}$ estão em correspondência um a um com o conjunto de partições de $n+1$. Em particular, o conjunto de órbitas nilpotentes é finito. Desse modo,

a) $\mathcal{O}_{\text {reg }}=\mathcal{O}_{[n+1]}$;

b) $\mathcal{O}_{\text {subreg }}=\mathcal{O}_{[n, 1]}$;

c) $\mathcal{O}_{\min }=\mathcal{O}_{\left[2,1^{n-1}\right]}$;

d) $\mathcal{O}_{\text {zero }}=\mathcal{O}_{\left[1^{n+1}\right]}$.

Definição 2.4.17 ([oGVAG04]). Seja $N=n+1$ e $q \in \mathbb{Z}_{>0}$. Para $0 \leq q \leq N$, seja $\lambda_{q}$ a partição de $N$ definida por

$$
\lambda_{q}:= \begin{cases}{\left[q^{r}, s\right]} & \text { se } N=q r+s \text { com } r, s \in \mathbb{Z}_{\geq 0} \text { e } 0 \leq s \leq q-1 \\ {[N]} & \text { se } q>N\end{cases}
$$

Teorema 2.4.18 (Teorema 5.17 em [Ara15a]). Seja $k$ um número admissível com denominador $q \in \mathbb{Z}_{>0}$. Então

$$
\mathcal{V}\left(I_{k}\right)=\left\{x \in \mathfrak{g} \mid(\operatorname{ad} x)^{2 q}=0\right\}=\overline{\mathcal{O}_{q}},
$$

em que $\mathcal{O}_{q}=\mathcal{O}_{\lambda_{q}}$ é a órbita nilpotente correspondente à partição $\lambda_{q}$.

Exemplo 2.4.19. As órbitas nilpotentes dos $\mathfrak{s l}_{6}$-módulos admissíveis são:

a) $\mathcal{O}_{q}=\mathcal{O}_{\text {reg }}$ se $q \geq 6$;

b) $\mathcal{O}_{5}=\mathcal{O}_{\text {subreg }}$;

c) $\mathcal{O}_{4}=\mathcal{O}_{[4,2]}$;

d) $\mathcal{O}_{3}=\mathcal{O}_{\left[3^{2}\right]}$;

e) $\mathcal{O}_{2}=\mathcal{O}_{\left[2^{3}\right]}$;

f) $\mathcal{O}_{1}=\mathcal{O}_{\text {zero }}$.

Com base nos Teoremas 2.4.16 e 2.4.18, para $n \geq 1$, obtemos imediatamente

$$
\mathcal{O}_{q}= \begin{cases}\mathcal{O}_{\text {reg }} & \text { se } q>n \\ \mathcal{O}_{\text {subreg }} & \text { se } q=n \\ \mathcal{O}_{\text {zero }} & \text { se } q=1\end{cases}
$$

Por outro lado,

$$
\mathcal{O}_{2}= \begin{cases}\mathcal{O}_{\left[2^{r}\right]}, \text { em que } r=\frac{n+1}{2} & \text { se } n \text { é ímpar } \\ \mathcal{O}_{\left[2^{r}, 1\right]}, \text { em que } r=\frac{n}{2} & \text { se } n \text { é par }\end{cases}
$$


Proposição 2.4.20. $\mathcal{O}_{q}=\mathcal{O}_{\min }$ se, e somente se, $n=q=2$ ou $n=1$ e $q \geq 2$.

Observação 2.4.21.

a) Para $\mathfrak{g}=\mathfrak{s l}_{2}$,

i) $\mathcal{O}_{q}=\mathcal{O}_{\text {min }}=\mathcal{O}_{\text {prin }}=\mathcal{O}_{[2]}$ para todo $q \geq 2$, em que $\operatorname{dim} \mathcal{O}_{2}=2$;

ii) $\mathcal{O}_{1}=\mathcal{O}_{\text {subreg }}=\mathcal{O}_{\text {zero }}=\mathcal{O}_{\left[1^{2}\right]}$, em que $\operatorname{dim} \mathcal{O}_{1}=0$.

b) Para $\mathfrak{g}=\mathfrak{s l}_{3}$,

i) $\mathcal{O}_{q}=\mathcal{O}_{\text {prin }}=\mathcal{O}_{[3]}$ para todo $q \geq 3$, em que $\operatorname{dim} \mathcal{O}_{3}=6$;

ii) $\mathcal{O}_{2}=\mathcal{O}_{\text {min }}=\mathcal{O}_{\text {subreg }}=\mathcal{O}_{[2,1]}$, em que $\operatorname{dim} \mathcal{O}_{2}=4$;

iii) $\mathcal{O}_{1}=\mathcal{O}_{\text {zero }}=\mathcal{O}_{\left[1^{3}\right]}$, em que $\operatorname{dim} \mathcal{O}_{1}=0$.

c) Para $\mathfrak{g}=\mathfrak{s l}_{4}$,

i) $\mathcal{O}_{q}=\mathcal{O}_{\text {prin }}=\mathcal{O}_{[4]}$ para todo $q \geq 4$, em que $\operatorname{dim} \mathcal{O}_{4}=12$;

ii) $\mathcal{O}_{3}=\mathcal{O}_{\text {subreg }}=\mathcal{O}_{[3,1]}$, em que $\operatorname{dim} \mathcal{O}_{3}=10$;

iii) $\mathcal{O}_{2}=\mathcal{O}_{\left[2^{2}\right]}$, em que $\operatorname{dim} \mathcal{O}_{\left[2^{2}\right]}=8$;

iv) $\mathcal{O}_{\min }=\mathcal{O}_{\left[2,1^{2}\right]}$, em que $\operatorname{dim} \mathcal{O}_{\min }=6$;

v) $\mathcal{O}_{1}=\mathcal{O}_{\text {zero }}=\mathcal{O}_{\left[1^{4}\right]}$, em que $\operatorname{dim} \mathcal{O}_{1}=0$.

Vamos definir o subconjunto de pesos admissíveis de nível $k$

$$
\operatorname{Pr}_{k, \mathbb{Z}}=\left\{\lambda \in \operatorname{Pr}_{k}:\left\langle\lambda, \alpha^{\vee}\right\rangle \in \mathbb{Z} \text { para } \alpha \in \Pi\right\}
$$

Então de acordo com [KW89], temos que

$$
\operatorname{Pr}_{k, \mathbb{Z}}=\left\{\lambda \in \widehat{\mathfrak{h}}^{*}: \lambda(c)=k,\left\langle\lambda, \alpha^{\vee}\right\rangle \in \mathbb{Z}_{\geq 0} \text { para } \alpha \in \Pi,\left\langle\lambda, \theta^{\vee}\right\rangle \leq p-h^{\vee}\right\},
$$

em que $p=\left(k+h^{\vee}\right) q$. Ainda, de acordo com [KW89] obtemos

$$
\operatorname{Pr}_{k}=\bigcup_{\substack{y \in \widetilde{W} \\ y\left(\widehat{\Delta}\left(k \Lambda_{0}\right)_{+}\right) \subset \widehat{\Delta}_{+}^{\mathrm{re}}}} \operatorname{Pr}_{k, y}, \quad \operatorname{Pr}_{k, y}=\left\{y \cdot \lambda: \lambda \in \operatorname{Pr}_{k, \mathbb{Z}}\right\},
$$

em que o grupo de Weyl afim estendido $\widetilde{W}$ de $\mathfrak{g}$ é definido como

$$
\widetilde{W}=W \ltimes P^{\vee} .
$$

Para $\mu \in P^{\vee}$, denotamos por $t_{\mu}$ o elemento correspondente de $\widetilde{W}$. A ação de $t_{\mu}$ em $\widetilde{\mathfrak{h}}^{*}$ por $\mu \in P^{\vee}$ é dada por

$$
t_{\mu}(\gamma)=\gamma+(\gamma, \delta) \mu-\left(\frac{(\alpha, \alpha)}{2}(\gamma, \delta)+(\gamma, \mu)\right) \delta
$$

para $\gamma \in \widetilde{\mathfrak{h}}^{*}$. Além disso, por $y, y^{\prime} \in \widetilde{W}$ satisfazendo $y\left(\widehat{\Delta}\left(k \Lambda_{0}\right)_{+}\right) \subset \widehat{\Delta}_{+}^{\text {re }}, y^{\prime}\left(\widehat{\Delta}\left(k \Lambda_{0}\right)_{+}\right) \subset \widehat{\Delta}_{+}^{\text {re }}$ temos

$$
\operatorname{Pr}_{k, y} \cap \operatorname{Pr}_{k, y^{\prime}} \neq \emptyset \quad \Longleftrightarrow \operatorname{Pr}_{k, y}=\operatorname{Pr}_{k, y^{\prime}} \quad \Longleftrightarrow \quad y^{\prime}=y t_{q \omega_{j}} w_{j}
$$


para algum $j \in\{1,2, \ldots, n\}$, em que $w_{j}$ é o único elemento d $W$ que preserva o conjunto $\left\{\alpha_{1}, \alpha_{2}, \ldots, \alpha_{n},-\theta\right\}$ e $w_{j}(-\theta)=\alpha_{j}$.

Proposição 2.4.22 (Proposição 2.8 e Proposição 2.10 em [AFR17]). Seja $k$ um número admissível para $\widehat{\mathfrak{g}}$ com denominador $q \in \mathbb{Z}_{>0}$. Temos

$$
\left[\overline{\operatorname{Pr}}_{k}\right]=\bigcup_{\substack{\eta \in P_{+}^{\vee} \\(\eta, \theta) \leq q-1}}\left[\overline{\operatorname{Pr}}_{k, t_{-\eta}}\right]
$$

onde $P_{+}^{\vee}$ é o conjunto de copesos dominantes. Em particular para $\mathfrak{g}=\mathfrak{s l}_{n+1}$

$$
\left[\overline{P r}_{k}^{\mathbb{Q}_{\text {min }}}\right]=\bigsqcup_{a=1}^{q-1}\left[\overline{\operatorname{Pr}}_{k, t_{-a \varpi_{1}}}\right]=\bigsqcup_{a=1}^{q-1}\left\{\left[\bar{\lambda}-\frac{a p}{q} \varpi_{1}\right] \mid \lambda \in \widehat{P}_{+}^{p-n-1}\right\}
$$




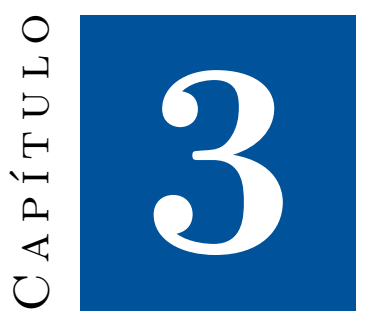

\section{Módulos de Gelfand-Tsetlin}

Neste capítulo, abordaremos os principais resultados referentes aos módulos de Gelfand-Tsetlin para a álgebra de Lie complexa $\mathfrak{s l}_{n}$. Porém, uma vez que historicamente o tratamento da teoria de módulos de Gelfand-Tsetlin foi desenvolvida para $\mathfrak{g l}_{n}$, começaremos estudando módulos para esta álgebra (veja seção 3.2). Destacamos ainda que, todo módulo de Gelfand-Tsetlin sobre $\mathfrak{g l}_{n}$ é um módulo de Gelfand-Tsetlin sobre $\mathfrak{s l}_{n}$. Reciprocamente, todo módulo de Gelfand-Tsetlin sobre $\mathfrak{s l}_{n}$ torna-se um $\mathfrak{g l}_{n}$-módulo de Gelfand-Tsetlin após definirmos a ação da matriz identidade de $\mathfrak{g l}_{n}$ como multiplicação por um escalar. Além disso, a irredutibilidade ou a falta dela é preservada.

Além dos conceitos e terminologias peculiares dessas duas famílias de módulos, apresentaremos a teoria de módulos de Gelfand-Tsetlin de relações desenvolvida em [FRZ19], onde são dadas realizações explícitas através das chamadas tabelas de Gelfand-Tsetlin para: módulos de relações simples de peso máximo e de dimensão finita, assim como módulos genéricos e módulos densos para $\mathfrak{g l}_{n}$. Ainda neste capítulo, estabeleceremos a classificação completa de $\mathfrak{s l}_{n}$-módulos de peso máximo de relações e $\mathfrak{s l}_{n}$-módulos induzidos de $\mathfrak{s l}_{2}$ de relações. Classificação que utilizaremos no próximo capítulo para realizarmos os módulos de peso máximo admissíveis, no qual se encontra o principal problema resolvido nesta tese. 


\subsection{Convenções}

Seja $\left\{E_{i j} \mid 1 \leq i, j \leq n\right\}$ a base padrão de $\mathfrak{g l}_{n}$ de matrizes elementares. De acordo com [FRZ19], para $\mathfrak{g l}_{n}$ escreveremos vetores em $\mathbb{C}^{\frac{n(n+1)}{2}}$ como

$$
L=\left(l_{i j}\right)=\left(l_{n 1}, \ldots, l_{n n}\left|l_{n-1,1}, \ldots, l_{n-1, n-1}\right| \cdots\left|l_{21}, l_{22}\right| l_{11}\right) .
$$

Denotamos $\mathbb{Z}_{0}^{\frac{n(n+1)}{2}}:=\left\{z \in \mathbb{Z}^{\frac{n(n+1)}{2}} \mid z_{n i}=0,1 \leq i \leq n\right\}$. Para $1 \leq j \leq i \leq n, \boldsymbol{\delta}^{i j} \in \mathbb{Z}_{0}^{\frac{n(n+1)}{2}}$ é definido por $\left(\boldsymbol{\delta}^{i j}\right)_{i j}=1$ e todos os outros $\left(\boldsymbol{\delta}^{i j}\right)_{k \ell}$ são zero.

A transposição do grupo simétrico $S_{N}$ que troca $i$ e $j$ será denotada por $(i, j)$. Definimos $G:=S_{n} \times S_{n-1} \times \cdots \times S_{1}$ e a componente $i$-ésima de $\sigma \in G$ será indicada por $\sigma[i]$. Para um anel comutativo $R$, por Specm $R$ denotamos o conjunto de ideais maximais de $R$.

\subsection{Módulos de Gelfand-Tsetlin para $\mathfrak{g l}_{n}$}

Nesta seção vamos resumir algumas definições e resultados relevantes sobre os módulos de Gelfand-Tsetlin.

Para qualquer bandeira $\mathcal{F}: \mathfrak{g l}_{1} \subset \cdots \subset \mathfrak{g l}_{n}$, temos uma bandeira induzida $U_{1} \subset \cdots \subset U_{n}$ das álgebras envolventes universais correspondentes, em que $U_{i}:=U\left(\mathfrak{g l}_{i}\right)$, para cada $i=1, \ldots, n$. Seja $\mathcal{Z}_{m}$ o centro de $U_{m}$. Seguindo [DFO94], chamamos a subálgebra $\Gamma_{\mathcal{F}}$ de $U:=U_{n}$ gerada por $\left\{\mathcal{Z}_{m} \mid m=1, \ldots, n\right\}$ a subálgebra de Gelfand-Tsetlin de $U$ (em relação a $\mathcal{F}$ ). Se a bandeira for dada por inclusões no canto superior esquerdo, chamaremos ela bandeira padrão e a subálgebra de Gelfand-Tsetlin correspondente será chamada de subálgebra de Gelfand-Tsetlin padrão e será indicada por $\Gamma_{s t}$.

Definição 3.2.1. Um U-módulo $M$ finitamente gerado é chamado de $\Gamma_{\mathcal{F}}$-módulo de GelfandTsetlin se $M$ pode ser decomposto em uma soma direta de $\Gamma_{\mathcal{F}}$-módulos:

$$
M=\bigoplus_{\mathrm{m} \in \operatorname{Specm} \Gamma_{\mathcal{F}}} M(\mathrm{~m})
$$

em que

$$
M(\mathrm{~m})=\left\{v \in M \mid \mathrm{m}^{k} v=0 \text { para algum } k \geq 0\right\} .
$$


Identificando $\mathrm{m}$ com o homomorfismo $\chi: \Gamma_{\mathcal{F}} \rightarrow \mathbb{C}$ com Ker $\chi=\mathrm{m}$, chamaremos $\mathrm{m}$ um caráter de Gelfand-Tsetlin de $M$ se $M(\mathrm{~m}) \neq 0$. O conjunto de todos os caracteres de Gelfand-Tsetlin de $M$ é o suporte de Gelfand-Tsetlin de $M$. Também $\operatorname{dim} M(\mathrm{~m})$ será chamado a multiplicidade de Gelfand-Tsetlin de m.

\subsection{Módulos de $\Gamma_{s t}$-relações para $\mathfrak{g l}_{n}$}

Nesta seção vamos relembrar os resultados sobre módulos de Gelfand-Tsetlin (para $\Gamma_{s t}$ ) apresentados por V. Futorny, L. E. Ramirez e J. Zhang em [FRZ19]. Neste trabalho, os autores fornecem bases explícitas para uma classe de $\mathfrak{g l}_{n}$-módulos o que nos permite estender a construção de Gelfand-Tsetlin para módulos mais gerais, os chamados $\mathfrak{g l}_{n}$-módulos de $\Gamma_{s t}$-relações ${ }^{1}$. Esses módulos possuem propriedades semelhantes aos módulos de dimensão finita (veja [GT50]) e aos módulos genéricos Gelfand-Tsetlin definidos em [DFO94]. Vamos então apresentar de modo sucinto essa construção feita em [FRZ19]. Para isso, vamos começar definindo os chamados conjuntos de relações admissíveis.

Definimos $\mathfrak{V}:=\{(i, j) \mid 1 \leq j \leq i \leq n\}$ e $\mathcal{R}:=\mathcal{R}^{-} \cup \mathcal{R}^{0} \cup \mathcal{R}^{+} \subset \mathfrak{V} \times \mathfrak{V}$, em que

$$
\begin{aligned}
& \mathcal{R}^{+}:=\{((i, j) ;(i-1, t)) \mid 2 \leq j \leq i \leq n, 1 \leq t \leq i-1\} \\
& \mathcal{R}^{-}:=\{((i, j) ;(i+1, s)) \mid 1 \leq j \leq i<n, 1 \leq s \leq i+1\} \\
& \mathcal{R}^{0}:=\{((n, i) ;(n, j)) \mid 1 \leq i \neq j \leq n\}
\end{aligned}
$$

Qualquer subconjunto $\mathcal{C} \subseteq \mathcal{R}$ será chamado de conjunto de relações. Com qualquer $\mathcal{C} \subseteq \mathcal{R}$, associamos um grafo orientado $G(\mathcal{C})$ com o conjunto de vértices $\mathfrak{V}$, que possui uma seta do vértice $(i, j)$ ao vértice $(r, s)$ se, e somente se, $((i, j) ;(r, s)) \in \mathcal{C}$. Por conveniência, representaremos o conjunto $\mathfrak{V}$ como uma tabela triangular com $n$ linhas, em que a $k$-ésima linha é $\{(k, 1), \ldots,(k, k)\}$, $k=1, \ldots, n$.

Definição 3.3.1 (Definição 4.1 em [FRZ19]). Seja $\mathcal{C}$ um conjunto de relações.

a) Denotaremos por $\mathfrak{V}(\mathcal{C}) \subseteq \mathfrak{V}$ o conjunto de todos os vértices em $G(\mathcal{C})$ que são vértices iniciais ou finais de uma flecha.

b) $O$ conjunto $\mathcal{C}$ é denominado indecomponivel se o grafo não orientado associado $G(\mathcal{C})$ é um grafo conexo.

\footnotetext{
${ }^{1}$ Observamos que originalmente estes são chamados de módulos admissíveis, mas lembramos que neste trabalho reservamos o termo admissível para certos módulos sobre a álgebra afim universal que iremos discutir no próximo capítulo.
} 
c) Diremos que $\mathcal{C}$ é um loop se $G(\mathcal{C})$ é um grafo circular orientado.

d) Sejam $(i, j),(r, s) \in \mathfrak{V}$, escreveremos $(i, j) \succeq_{\mathcal{C}}(r, s)$ se existir um caminho orientado em $G(\mathcal{C})$ que começa em $(i, j)$ e termina em $(r, s)$.

Para $v \in \mathbb{C}^{\frac{n(n+1)}{2}}$ denotamos por $T(v)$ a imagem de $v$ pelo isomorfismo natural entre $\mathbb{C}^{\frac{n(n+1)}{2}}$ e $\mathbb{C}^{n} \times \cdots \times \mathbb{C}^{1}$. Se $T(v)=\left(v^{(n)}, \ldots, v^{(1)}\right)$, então nos referimos a $v^{(k)}=\left(v_{k 1}, \ldots, v_{k k}\right)$ como a $k$-ésima linha de $T(v)$. Portanto, podemos imaginar $T(v)$ como uma tabela triangular de altura $n$.

Definição 3.3.2. A seguinte tabela $T(v)$ com entradas complexas $\left\{v_{i j}: 1 \leq j \leq i \leq n\right\}$

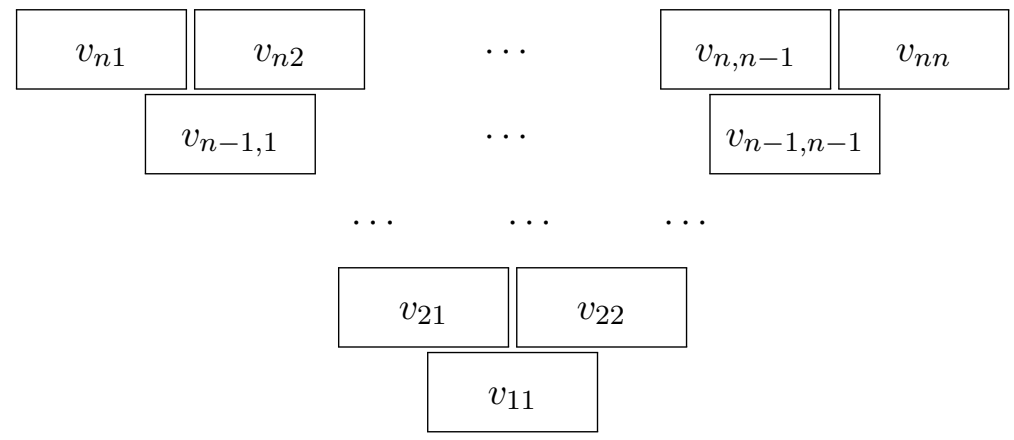

é chamada de tabela de Gelfand-Tsetlin de altura n.

Observação 3.3.3. É importante distinguir $v$ e $T(v)$, pois são vetores em espaços vetoriais não isomorfos. Uma importante diferença é que a soma de duas tabelas não é outra tabela.

Exemplo 3.3.4. Seja $v=\left(v_{44}, v_{43}, v_{42}, v_{41}, v_{33}, v_{32}, v_{31}, v_{22}, v_{21}, v_{11}\right)$. A tabela $T(v)$ é

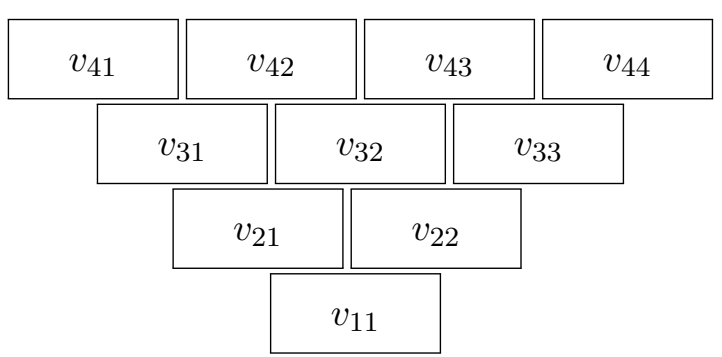

Assim, temos que $T\left(v+\ell \delta^{11}+m \delta^{21}+n \delta^{22}+r \delta^{31}+s \delta^{32}+t \delta^{33}\right)$ representa a tabela ${ }^{2}$

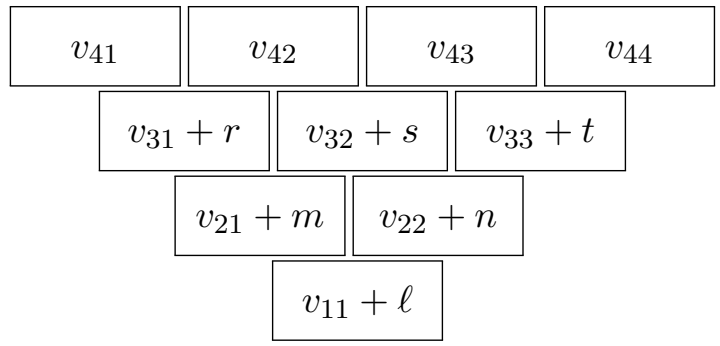

Em que $(\ell, m, n, r, s, t) \in \mathbb{Z}^{6}$.

Definição 3.3.5. Um vetor $v$ e a tabela de Gelfand-Tsetlin $T(v)$ de altura $n$ são chamados

a) Standard: se $v_{k i}-v_{k-1, i} \in \mathbb{Z}_{\geq 0} \quad$ e $v_{k-1, i}-v_{k, i+1} \in \mathbb{Z}_{>0}$, para todo $1 \leq i \leq k \leq n-1$.

b) Genérica: se $v_{k i}-v_{k j} \notin \mathbb{Z}$ para todo $1 \leq i<j \leq k \leq n-1$.

\footnotetext{
${ }^{2}$ Lembramos que $\delta^{i j} \in \mathbb{Z}_{0}^{10}$ é definido por $\left(\delta^{i j}\right)_{i j}=1$ e todos os outros $\left(\delta^{i j}\right)_{k \ell}$ são zero.
} 
c) Fortemente genérica: se $v_{k i}-v_{k j} \notin \mathbb{Z}$ para qualquer $1 \leq i<j \leq k \leq n$.

d) Não crítica: se $v_{k i} \neq v_{k j}$ para todo $1 \leq i<j \leq k \leq n-1$, e crítica caso contrário.

No artigo [FRZ19] os autores observam que $\mathcal{C} \subseteq \mathcal{R}$ pode ser decomposto na forma $\mathcal{C}=\mathcal{C}^{-} \cup \mathcal{C}^{0} \cup \mathcal{C}^{+}$, em que $\mathcal{C}^{-}:=\mathcal{R}^{-} \cap \mathcal{C}, \mathcal{C}^{0}:=\mathcal{R}^{0} \cap \mathcal{C}$ e $\mathcal{C}^{+}:=\mathcal{R}^{+} \cap \mathcal{C}$. Decomposição que permite provar que os conjuntos de relações que estamos considerando são generalizações dos conjuntos de relações standard usados no Teorema de Gelfand-Tsetlin em [GT88]. Mas antes disso precisamos da seguinte definição:

Definição 3.3.6 (Definição $4.2 \mathrm{em}$ [FRZ19]). Seja $\mathcal{C}$ um conjunto de relações e $T(v)$ qualquer tabela de Gelfand-Tsetlin, em que $v=\left(v_{i j}\right) \in \mathbb{C}^{\frac{n(n+1)}{2}}$.

a) Dizemos que $T(v)$ satisfaz $\mathcal{C}$ se:

(i) $v_{i j}-v_{r s} \in \mathbb{Z}_{\geq 0}$ para qualquer $((i, j) ;(r, s)) \in \mathcal{C} \cap\left(\mathcal{R}^{+} \cup \mathcal{R}^{0}\right)$;

(ii) $v_{i j}-v_{r s} \in \mathbb{Z}_{>0}$ para qualquer $((i, j) ;(r, s)) \in \mathcal{C} \cap \mathcal{R}^{-}$.

b) Dizemos que $T(v)$ é uma $\mathcal{C}$-realização se $T(v)$ satisfizer $\mathcal{C}$ e para qualquer $1 \leq k<n$, temos $v_{k i}-v_{k j} \in \mathbb{Z}$ se, e somente se, $(k, i)$ e $(k, j)$ estão na mesma componente conexa de $G(\mathcal{C})$.

c) Suponhamos que $T(v)$ satisfaça $\mathcal{C}$. Então $\mathcal{B}_{\mathcal{C}}(T(v))$ indica o conjunto de todas as tabelas da forma $T(v+z), z \in \mathbb{Z}_{0}^{\frac{n(n+1)}{2}}$ satisfazendo $\mathcal{C}$. Além disso, $V_{\mathcal{C}}(T(v))$ indica o espaço vetorial complexo com base $\mathcal{B}_{\mathcal{C}}(T(v))$.

Exemplo 3.3.7. Uma tabela $T(v)$ é standard se, e somente se, $T(v)$ satisfizer o conjunto de relações $\mathcal{S}=\mathcal{S}^{+} \cup \mathcal{S}^{-}$em que

$$
\begin{aligned}
& \left.\mathcal{S}^{+}:=\{(i+1, j) ;(i, j)) \mid 1 \leq j \leq i<n\right\} \\
& \mathcal{S}^{-}:=\{((i, j) ;(i+1, j+1)) \mid 1 \leq j \leq i<n\}
\end{aligned}
$$

Observação 3.3.8. Observamos que se $T(v)$ satisfaz $\mathcal{C}$ e $\mathcal{C}$ é o conjunto máximo de relações satisfeitas por $T(v)$, então $T(v)$ é uma $\mathcal{C}$-realização. Em particular, toda tabela de Gelfand-Tsetlin satisfaz $\emptyset \mathrm{e}$ $T(v)$ é uma realização de $\emptyset$ se, e somente se, $v_{k i}-v_{k j} \notin \mathbb{Z}$ para qualquer $1 \leq k \leq n-1$.

Definição 3.3.9 (Definição 4.4 em [FRZ19]). Seja $\mathcal{C}$ um conjunto de relações. Chamamos $\mathcal{C}$ admissivel se para qualquer $\mathcal{C}$-realização $T(v), V_{\mathcal{C}}(T(v))$ tem uma estrutura de $\mathfrak{g l}_{n}$-módulo, munido com a seguinte ação dos geradores de $\mathfrak{g l}_{n}$ :

$$
\begin{array}{r}
E_{k, k+1}(T(w))=-\sum_{i=1}^{k}\left(\frac{\prod_{j=1}^{k+1}\left(w_{k i}-w_{k+1, j}\right)}{\prod_{j \neq i}^{k}\left(w_{k i}-w_{k j}\right)}\right) T\left(w+\delta^{k i}\right), \\
E_{k+1, k}(T(w))=\sum_{i=1}^{k}\left(\frac{\prod_{j=1}^{k-1}\left(w_{k i}-w_{k-1, j}\right)}{\prod_{j \neq i}^{k}\left(w_{k i}-w_{k j}\right)}\right) T\left(w-\delta^{k i}\right), \\
E_{k, k}(T(w))=\left(k-1+\sum_{i=1}^{k} w_{k i}-\sum_{i=1}^{k-1} w_{k-1, i}\right) T(w) .
\end{array}
$$

Neste caso diremos que $V_{\mathcal{C}}(T(v))$ é um $\mathfrak{g l}_{n}$-módulo de $\Gamma_{s t}$-relações. 
Um resultado importante é que um módulo de $\Gamma_{s t}$-relações é um módulo de Gelfand-Tsetlin. Em particular, temos o seguinte.

Teorema 3.3.10 (Capitulo IX, Secção 59 em [Zel73]).

1. $\Gamma_{\text {st }}$ é gerada por $\left\{c_{m k} \mid k=1, \ldots, m\right\}$, onde

$$
c_{m k}=\sum_{\left(i_{1}, \ldots, i_{k}\right) \in\{1, \ldots, m\}^{k}} E_{i_{1} i_{2}} E_{i_{2} i_{3}} \ldots E_{i_{k} i_{1}} .
$$

2. A ação dos geradores de $\Gamma_{\text {st }}$ no módulo de dimensão finita $L(\lambda)$ é dada pelas seguintes fórmulas:

$$
c_{m k}(T(v))=\gamma_{m k}(v) T(v),
$$

em que

$$
\gamma_{m k}(v):=\sum_{i=1}^{m}\left(v_{m i}+m-1\right)^{k} \prod_{j \neq i}^{m}\left(1-\frac{1}{v_{m i}-v_{m j}}\right),
$$

com a função geradora

$$
1-\sum_{k=0}^{\infty} \gamma_{m k}(v) u^{-k-1}=\prod_{i=1}^{m} \frac{u-v_{m i}-m}{u-v_{m i}-m+1} .
$$

Observação 3.3.11. Para cada $m \in\{1,2, \ldots, n\}$, os polinômios $\gamma_{m k}(v)$ são simétricos de grau $k$ nas variáveis $v_{m 1}, v_{m 2}, \ldots, v_{m m}$ e $\left\{\gamma_{m 1}(v), \gamma_{m 2}(v), \ldots, \gamma_{m m}(v)\right\}$ geram a álgebra de polinômios $S_{m}$-invariantes nas variáveis $v_{m 1}, v_{m 2}, \ldots, v_{m m}$ (veja [Zel73]).

Exemplo 3.3.12. Vamos escrever explicitamente as expressões em (3.1) e escrever as fórmulas de Gelfand-Tsetlin no caso de $\mathfrak{g l}_{4}$ para qualquer $T(w) \in \mathcal{B}_{\mathcal{C}}(T(v))$.

- $E_{11}(T(w))=w_{11} T(w)$

- $E_{22}(T(w))=\left(w_{21}+w_{22}-w_{11}+1\right) T(w)$

- $E_{33}(T(w))=\left(w_{31}+w_{32}+w_{33}-\left(w_{21}+w_{22}\right)+2\right) T(w)$

- $E_{44}(T(w))=\left(w_{41}+w_{42}+w_{43}+w_{44}-\left(w_{31}+w_{32}+w_{33}\right)+3\right) T(w)$

- $E_{12}(T(w))=-\left(w_{11}-w_{21}\right)\left(w_{11}-w_{22}\right) T\left(w+\delta^{11}\right)$

- $E_{21}(T(w))=T\left(w-\delta^{11}\right)$

$$
\begin{aligned}
E_{23}(T(w)) & =\frac{\left(w_{21}-w_{31}\right)\left(w_{21}-w_{32}\right)\left(w_{21}-w_{33}\right)}{\left.w_{22}-w_{21}\right)\left(w+w_{22}-w_{33}\right)} T\left(w+\delta^{21}\right) \\
& +\frac{\left(w_{22}-w_{31}\right)\left(w_{22}-w_{32}\right)\left(w_{22}\right)}{w_{21}-w_{22}} T(w)
\end{aligned}
$$

- $E_{32}(T(w))=\frac{w_{21}-w_{11}}{w_{21}-w_{22}} T\left(w-\delta^{21}\right)+\frac{w_{22}-w_{11}}{w_{22}-w_{21}} T\left(w-\delta^{22}\right)$ 


$$
\begin{aligned}
& E_{34}(T(w))=-\frac{\left(w_{31}-w_{41}\right)\left(w_{31}-w_{42}\right)\left(w_{31}-w_{43}\right)\left(w_{31}-w_{44}\right)}{\left(w_{31}-w_{32}\right)\left(w_{31}-w_{33}\right)} T\left(w+\delta^{31}\right) \\
& -\frac{\left(w_{32}-w_{41}\right)\left(w_{32}-w_{42}\right)\left(w_{32}-w_{43}\right)\left(w_{32}-w_{44}\right)}{\left(w_{32}-w_{31}\right)\left(w_{32}-w_{33}\right)} T\left(w+\delta^{32}\right) \\
& -\frac{\left(w_{33}-w_{41}\right)\left(w_{33}-w_{42}\right)\left(w_{33}-w_{43}\right)\left(w_{33}-w_{44}\right)}{\left(w_{33}-w_{31}\right)\left(w_{33}-w_{32}\right)} T\left(w+\delta^{33}\right) \\
& E_{43}(T(w))=\frac{\left(w_{31}-w_{21}\right)\left(w_{31}-w_{22}\right)}{\left(w_{31}-w_{32}\right)\left(w_{31}-w_{33}\right)} T\left(w-\delta^{31}\right)+\frac{\left(w_{32}-w_{21}\right)\left(w_{32}-w_{22}\right)}{\left(w_{32}-w_{31}\right)\left(w_{32}-w_{33}\right)} T\left(w-\delta^{32}\right) \\
& +\frac{\left(w_{33}-w_{21}\right)\left(w_{33}-w_{22}\right)}{\left(w_{33}-w_{31}\right)\left(w_{33}-w_{32}\right)} T\left(w-\delta^{33}\right) \\
& E_{13}(T(w))=\frac{\left(w_{22}-w_{11}\right)\left(w_{21}-w_{31}\right)\left(w_{21}-w_{32}\right)\left(w_{21}-w_{33}\right)}{w_{21}-w_{22}} T\left(w+\delta^{11}+\delta^{21}\right) \\
& +\frac{\left(w_{21}-w_{11}\right)\left(w_{22}-w_{31}\right)\left(w_{22}-w_{32}\right)\left(w_{22}-w_{33}\right)}{w_{22}-w_{21}} T\left(w+\delta^{11}+\delta^{22}\right) \\
& \text { - } E_{31}(T(w))=\frac{1}{w_{21}-w_{22}} T\left(w-\delta^{11}-\delta^{21}\right)+\frac{1}{w_{22}-w_{21}} T\left(w-\delta^{11}-\delta^{22}\right) \\
& E_{24}(T(w))=\frac{\left(w_{21}-w_{32}\right)\left(w_{21}-w_{33}\right) \prod_{j=1}^{4}\left(w_{31}-w_{4 j}\right)}{\left(w_{22}-w_{21}\right)\left(w_{31}-w_{32}\right)\left(w_{31}-w_{33}\right)} T\left(w+\delta^{21}+\delta^{31}\right) \\
& +\frac{\left(w_{21}-w_{31}\right)\left(w_{21}-w_{33}\right) \prod_{j=1}^{4}\left(w_{32}-w_{4 j}\right)}{\left(w_{22}-w_{21}\right)\left(w_{32}-w_{31}\right)\left(w_{32}-w_{33}\right)} T\left(w+\delta^{21}+\delta^{32}\right) \\
& +\frac{\left(w_{21}-w_{31}\right)\left(w_{21}-w_{32}\right) \prod_{j=1}^{4}\left(w_{33}-w_{4 j}\right)}{\left(w_{22}-w_{21}\right)\left(w_{33}-w_{31}\right)\left(w_{33}-w_{32}\right)} T\left(w+\delta^{21}+\delta^{33}\right) \\
& +\frac{\left(w_{22}-w_{32}\right)\left(w_{22}-w_{33}\right) \prod_{j=1}^{4}\left(w_{31}-w_{4 j}\right)}{\left(w_{21}-w_{22}\right)\left(w_{31}-w_{32}\right)\left(w_{31}-w_{33}\right)} T\left(w+\delta^{22}+\delta^{31}\right) \\
& +\frac{\left(w_{22}-w_{31}\right)\left(w_{22}-w_{33}\right) \prod_{j=1}^{4}\left(w_{32}-w_{4 j}\right)}{\left(w_{21}-w_{22}\right)\left(w_{32}-w_{31}\right)\left(w_{32}-w_{33}\right)} T\left(w+\delta^{22}+\delta^{32}\right) \\
& +\frac{\left(w_{22}-w_{31}\right)\left(w_{22}-w_{32}\right) \prod_{j=1}^{4}\left(w_{33}-w_{4 j}\right)}{\left(w_{21}-w_{22}\right)\left(w_{33}-w_{31}\right)\left(w_{33}-w_{32}\right)} T\left(w+\delta^{22}+\delta^{33}\right) \\
& E_{42}(T(w))=\frac{\left(w_{21}-w_{11}\right)\left(w_{31}-w_{22}\right)}{\left(w_{21}-w_{22}\right)\left(w_{31}-w_{32}\right)\left(w_{31}-w_{33}\right)} T\left(w-\delta^{21}-\delta^{31}\right) \\
& +\frac{\left(w_{21}-w_{11}\right)\left(w_{32}-w_{22}\right)}{\left(w_{21}-w_{22}\right)\left(w_{32}-w_{31}\right)\left(w_{32}-w_{33}\right)} T\left(w-\delta^{21}-\delta^{32}\right) \\
& +\frac{\left(w_{21}-w_{11}\right)\left(w_{33}-w_{22}\right)}{\left(w_{21}-w_{22}\right)\left(w_{33}-w_{31}\right)\left(w_{33}-w_{32}\right)} T\left(w-\delta^{21}-\delta^{33}\right) \\
& +\frac{\left(w_{22}-w_{11}\right)\left(w_{31}-w_{21}\right)}{\left(w_{22}-w_{21}\right)\left(w_{31}-w_{32}\right)\left(w_{31}-w_{33}\right)} T\left(w-\delta^{22}-\delta^{31}\right) \\
& +\frac{\left(w_{22}-w_{11}\right)\left(w_{32}-w_{21}\right)}{\left(w_{22}-w_{21}\right)\left(w_{32}-w_{31}\right)\left(w_{32}-w_{33}\right)} T\left(w-\delta^{22}-\delta^{32}\right) \\
& +\frac{\left(w_{22}-w_{11}\right)\left(w_{33}-w_{21}\right)}{\left(w_{22}-w_{21}\right)\left(w_{33}-w_{31}\right)\left(w_{33}-w_{32}\right)} T\left(w-\delta^{22}-\delta^{33}\right)
\end{aligned}
$$




$$
\begin{aligned}
E_{14}(T(w))= & \frac{\left(w_{11}-w_{22}\right)\left(w_{21}-w_{32}\right)\left(w_{21}-w_{33}\right) \prod_{j=1}^{4}\left(w_{31}-w_{4 j}\right)}{\left(w_{22}-w_{21}\right)\left(w_{31}-w_{32}\right)\left(w_{31}-w_{33}\right)} T\left(w+\delta^{11}+\delta^{21}+\delta^{31}\right) \\
+ & \frac{\left(w_{11}-w_{22}\right)\left(w_{21}-w_{31}\right)\left(w_{21}-w_{33}\right) \prod_{j=1}^{4}\left(w_{32}-w_{4 j}\right)}{\left(w_{11}-w_{22}\right)\left(w_{22}-w_{21}\right)\left(w_{32}-w_{31}\right)\left(w_{32}-w_{33}\right)} T\left(w+\delta^{11}+\delta^{21}+\delta^{32}\right) \\
+ & \frac{\left(w_{11}-w_{22}\right)\left(w_{21}-w_{31}\right)\left(w_{21}-w_{32}\right) \prod_{j=1}^{4}\left(w_{33}-w_{4 j}\right)}{\left(w_{22}-w_{21}\right)\left(w_{33}-w_{31}\right)\left(w_{33}-w_{32}\right)} T\left(w+\delta^{11}+\delta^{21}+\delta^{33}\right) \\
+ & \frac{\left(w_{11}-w_{21}\right)\left(w_{22}-w_{32}\right)\left(w_{22}-w_{33}\right) \prod_{j=1}^{4}\left(w_{31}-w_{4 j}\right)}{\left(w_{21}-w_{22}\right)\left(w_{31}-w_{32}\right)\left(w_{31}-w_{33}\right)} T\left(w+\delta^{11}+\delta^{22}+\delta^{31}\right) \\
+ & \frac{\left(w_{11}-w_{21}\right)\left(w_{22}-w_{31}\right)\left(w_{22}-w_{33}\right) \prod_{j=1}^{4}\left(w_{32}-w_{4 j}\right)}{\left(w_{21}-w_{22}\right)\left(w_{32}-w_{31}\right)\left(w_{32}-w_{33}\right)} T\left(w+\delta^{11}+\delta^{22}+\delta^{32}\right) \\
+ & \frac{\left(w_{11}-w_{21}\right)\left(w_{22}-w_{31}\right)\left(w_{22}-w_{32}\right) \prod_{j=1}^{4}\left(w_{33}-w_{4 j}\right)}{\left(w_{21}-w_{22}\right)\left(w_{33}-w_{31}\right)\left(w_{33}-w_{32}\right)} T\left(w+\delta^{11}+\delta^{22}+\delta^{33}\right)
\end{aligned}
$$

$$
\begin{aligned}
E_{41}(T(w)) & =\frac{w_{31}-w_{22}}{\left(w_{21}-w_{22}\right)\left(w_{31}-w_{32}\right)\left(w_{31}-w_{33}\right)} T\left(w-\delta^{11}-\delta^{21}-\delta^{31}\right) \\
& +\frac{w_{32}-w_{22}}{\left(w_{21}-w_{22}\right)\left(w_{32}-w_{31}\right)\left(w_{32}-w_{33}\right)} T\left(w-\delta^{11}-\delta^{21}-\delta^{32}\right) \\
& +\frac{w_{33}-w_{22}}{\left(w_{21}-w_{22}\right)\left(w_{33}-w_{31}\right)\left(w_{33}-w_{32}\right)} T\left(w-\delta^{11}-\delta^{21}-\delta^{33}\right) \\
& +\frac{w_{31}-w_{21}}{\left(w_{22}-w_{21}\right)\left(w_{31}-w_{32}\right)\left(w_{31}-w_{33}\right)} T\left(w-\delta^{11}-\delta^{22}-\delta^{31}\right) \\
& +\frac{w_{32}-w_{21}}{\left(w_{22}-w_{21}\right)\left(w_{32}-w_{31}\right)\left(w_{32}-w_{33}\right)} T\left(w-\delta^{11}-\delta^{22}-\delta^{32}\right) \\
& +\frac{w_{33}-w_{21}}{\left(w_{22}-w_{21}\right)\left(w_{33}-w_{31}\right)\left(w_{33}-w_{32}\right)} T\left(w-\delta^{11}-\delta^{22}-\delta^{33}\right)
\end{aligned}
$$

Exemplo 3.3.13. Os polinômios $\gamma_{m k}(w)$ para $m \leq 4$ podem ser escritos explicitamente do seguinte modo:

- $\gamma_{11}(w)=w_{11}$

- $\gamma_{21}(w)=w_{21}+w_{22}+1$

- $\gamma_{22}(w)=w_{21}^{2}+w_{22}^{2}+w_{21}+w_{22}$

- $\gamma_{31}(w)=w_{31}+w_{32}+w_{33}+3$

- $\gamma_{32}(w)=w_{31}^{2}+w_{32}^{2}+w_{33}^{2}+2\left(w_{31}+w_{32}+w_{33}\right)+1$

- $\gamma_{33}(w)=w_{31}^{3}+w_{32}^{3}+w_{33}^{3}+4\left(w_{31}^{2}+w_{32}^{2}+w_{33}^{2}\right)-\left(w_{31} w_{32}+w_{31} w_{33}+w_{32} w_{33}\right)+w_{31}+w_{32}+w_{33}-6$

- $\gamma_{41}(w)=w_{41}+w_{42}+w_{43}+w_{44}+6$

- $\gamma_{42}(w)=w_{41}^{2}+w_{2}^{42}+w_{43}^{2}+w_{44}^{2}+3\left(w_{41}+w_{42}+w_{43}+w_{44}\right)+4$

- $\gamma_{43}(w)=w_{41}^{3}+w_{42}^{3}+w_{43}^{3}+w_{44}^{3}-\left(w_{41} w_{42}+w_{41} w_{43}+w_{41} w_{44}+w_{42} w_{43}+w_{42} w_{44}+w_{43} w_{44}\right)+$ $6\left(w_{41}^{2}+w_{42}^{2}+w_{43}^{2}+w_{44}^{2}\right)+3\left(w_{41}+w_{42}+w_{43}+w_{44}\right)-19$

- $\gamma_{44}(w)=w_{41}^{4}+w_{42}^{4}+w_{43}^{3}+w_{44}^{4}+9\left(w_{41}^{3}+w_{42}^{3}+w_{43}^{3}+w_{44}^{3}\right)+21\left(w_{41}^{2}+w_{42}^{2}+w_{31}^{2}+w_{44}^{2}\right)-$ $\left(w_{41} w_{42}^{2}+w_{41} w_{43}^{2}+w_{41} w_{44}^{2}+w_{42} w_{41}^{2}+w_{42} w_{43}^{2}+w_{42} w_{44}^{2}+w_{43} w_{41}^{2}+w_{43} w_{42}^{2}+w_{43} w_{44}^{2}+w_{44} w_{41}^{2}+\right.$ $\left.w_{44} w_{42}^{2}+w_{44} w_{43}^{2}\right)-10\left(w_{41} w_{42}+w_{41} w_{43}+w_{41} w_{44}+w_{42} w_{43}+w_{42} w_{44}+w_{43} w_{44}\right)-19\left(w_{41}+\right.$ $\left.w_{42}+w_{43}+w_{44}\right)-120$ 
No decorrer desta seção, lembramos as definições e teoremas relativos aos módulos GelfandTsetlin genéricos e singulares (não críticos) com realização por tabelas. Isto é, determinamos para quais conjuntos de relações $\mathcal{C}$ e tabelas $T(v)$ podemos definir uma estrutura de $\mathfrak{g l}_{n}$-módulo em $V_{\mathcal{C}}(T(v))$ com a ação de $\mathfrak{g l}_{n}$ dada pelas fórmulas de Gelfand-Tsetlin. Para detalhes, veja [FRZ19].

Ainda em [FRZ19], os autores estabelecem as condições necessárias e suficientes para garantir a admissibilidade do conjunto de relações $\mathcal{C}$. Mas antes eles definem conjuntos pré-admissíveis uma vez que os autores provam que para construir $\mathfrak{g l}_{n}$-módulos de $\Gamma_{s t}$-relações é preciso considerar unicamente este tipo de conjunto, temos assim as seguintes definições:

Definição 3.3.14 (Definição 4.14 em [FRZ19]). Seja $\mathcal{C}$ um subconjunto de $\mathcal{R}$. Chamamos $\mathcal{C}$ não crítico se para qualquer $\mathcal{C}$-realização $T(v)$, temos que $l_{k i} \neq l_{k j}, 1 \leq k \leq n-1, i \neq j$, $(k, i),(k, j) \in \mathfrak{V}(\mathcal{C})$.

Definição 3.3.15 (Definição 4.18 em [FRZ19]). Seja $\mathcal{C}$ qualquer conjunto de relações não críticas. Chamamos $\mathcal{C}$ de reduzido se para cada $(k, j) \in \mathfrak{V}(\mathcal{C})$ as seguintes condições são satisfeitas:

a) Existe no máximo um $i$ tal que $((k, j) ;(k+1, i)) \in \mathcal{C}$.

b) Existe no máximo um i tal que $((k+1, i) ;(k, j)) \in \mathcal{C}$.

c) Existe no máximo um i tal que $((k, j) ;(k-1, i)) \in \mathcal{C}$.

d) Existe no máximo um i tal que $((k-1, i) ;(k, j)) \in \mathcal{C}$.

e) Nenhuma relação na linha superior segue de outras relações.

Definição 3.3.16 (Definição 4.23 em [FRZ19]). Seja $\mathcal{C}$ um subconjunto não crítico indecomponível de $\mathcal{R}$. Um subconjunto $\mathcal{C}$ da forma $\{((k, i) ;(k+1, t)),((k+1, s) ;(k, j))\}$ com $i<j$ e $s<t$ será chamado de cruz. O grafo associado é o seguinte:

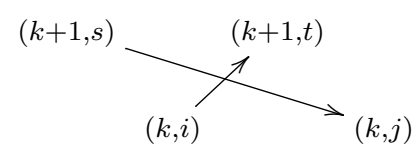

Definição 3.3.17 (Definição 4.27 em [FRZ19]). Seja $\mathcal{C}$ um conjunto indecomponível. Dizemos que $\mathcal{C}$ é pré-admissível se satisfaz as seguintes condiçôes:

a) $\mathcal{C}$ não contém loops.

b) $\mathcal{C}$ não é crítico.

c) Para cada $1 \leq k \leq n,(k, i) \succeq_{\mathcal{C}}(k, j)$ se, e somente se, $(k, i),(k, j)$ estão no mesmo subconjunto indecomponível $\mathcal{C}$ e $i<j$.

d) $\mathcal{C}$ é reduzido.

e) Não existem cruzes em $\mathcal{C}$.

Um conjunto arbitrário $\mathcal{C}$ é pré-admissivel se todo subconjunto indecomponível de $\mathcal{C}$ é préadmissível. 
Lembramos que $\{(k, i),(k, j)\} \subset \mathfrak{V}$ é um par adjunto para um grafo $G$ se $i<j$, existe um caminho em $G$ de $(k, i)$ a $(k, j)$ e não existe caminho em $G$ de $(k, i)$ a $(k, j)$ passando por $(k, t)$ com $i<t<j$.

Teorema 3.3.18 (Teorema 4.33 em [FRZ19]). Suponhamos que $\mathcal{C}$ seja um conjunto de relações pré-admissivel cujo grafo associado é $G=G(\mathcal{C})$. O conjunto $\mathcal{C}$ é admissivel se, e somente se, $G$ é uma união de grafos conexos que satisfaz a seguinte condição:

$\diamond$-Condição: Para cada par adjunto $\{(k, i),(k, j)\}, 1 \leq k<n$ existem $p, q$, tais que $\mathcal{C}_{1} \subseteq \mathcal{C}$ ou existem $s<t$, tais que $\mathcal{C}_{2} \subseteq \mathcal{C}$ em que os grafos associados a $\mathcal{C}_{1}$ e $\mathcal{C}_{2}$ são os seguintes
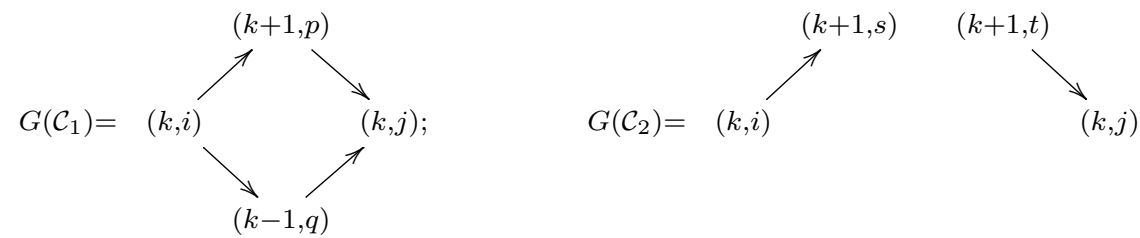

A partir deste momento, assumiremos que $\mathcal{C}$ é um conjunto de relações admissível e consideraremos o $\mathfrak{g l}_{n}$-módulo $V_{\mathcal{C}}(T(v))$. O critério de simplicidade para $V_{\mathcal{C}}(T(v))$ é o seguinte.

Teorema 3.3.19 (Teorema 5.6 em [FRZ19]). O módulo de Gelfand-Tsetlin $V_{\mathcal{C}}(T(v))$ é simples se, e somente se, $\mathcal{C}$ é o conjunto maximal (admissível) de relações satisfeito por $T(v)$.

Na descrição das bases vamos usar a seguinte notação:

Notação 3.3.20. Se $\mathcal{B}$ é um conjunto de desigualdades inteiras em $l, m, n, r, s, t$ vamos denotar por $M(\mathcal{B})$ o $\mathfrak{g l}_{n}$-módulo gerado (aplicando as fórmulas de Gelfand-Tsetlin) pelo conjunto de tabelas $\left\{T\left(v+\ell \delta^{11}+m \delta^{21}+n \delta^{22}+r \delta^{31}+s \delta^{32}+t \delta^{33}\right): l, m, n, r, s, t\right.$ satisfazem as desigualdades em $\left.\mathcal{B}\right\}$. Se $M(\mathcal{B})$ é irredutível gerado pela tabela $T(v)$, vamos denotar tal módulo por $L(\mathcal{B})$ ou por $L(T(v): \mathcal{B})$ se queremos destacar um gerador $T(v)$.

Exemplo 3.3.21. Seja $\mathcal{C}$ o conjunto de relações admissível com grafo associado
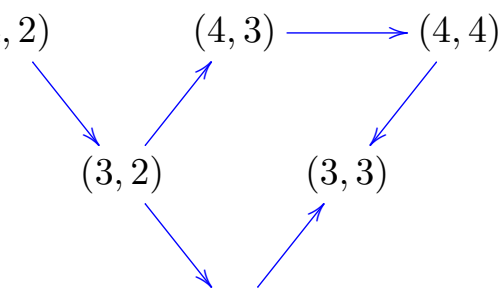

$(2,1)$

$(2,2)$

$(1,1)$

e $T(v)$ a tabela seguinte

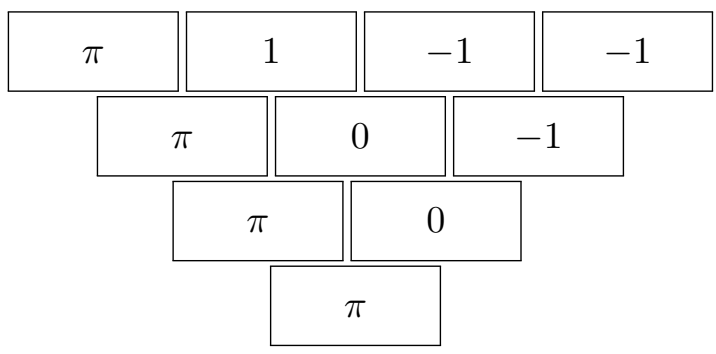


Lembramos que, $V_{\mathcal{C}}(T(v))=\operatorname{Span}_{\mathbb{C}} \mathcal{B}_{\mathcal{C}}(T(v))$, em que

$\mathcal{B}_{\mathcal{C}}(T(v))=\left\{T\left(v+\ell \delta^{11}+m \delta^{21}+n \delta^{22}+r \delta^{31}+s \delta^{32}+t \delta^{33}\right) \mid \ell, r \in \mathbb{Z}, m \leq r,-1 \leq s \leq 1, t \leq 0, t \leq n \leq s\right\}$.

é a base do módulo, a qual denotaremos simplesmente por:

$$
M\left(\left\{\begin{array}{c}
m \leq r \\
-1 \leq s \leq 1 \\
t \leq 0 \\
t \leq n \leq s \\
\ell, r \in \mathbb{Z}
\end{array}\right)\right.
$$

Então $T(v)$ satisfaz $\mathcal{C}$ e $V_{\mathcal{C}}(T(v))$ é um $\mathfrak{g l}_{4}$-módulo que não é simples em virtude do Teorema 3.3.19, dado que $\mathcal{C}$ não é o conjunto maximal de relações satisfeito por $T(v)$.

A seguir descrevemos os subquocientes irredutíveis de $V_{\mathcal{C}}(T(v))$ :

- Seja $\mathcal{C}_{1}$ o conjunto de relações admissível associado ao grafo

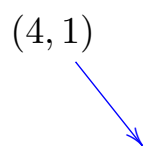

$(3,1)$
$(4,2)$

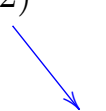

$(3,2)$

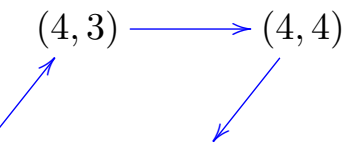

$(3,3)$

$(2,1)$

$(1,1)$

Assim $V_{\mathcal{C}_{1}}(T(v))$ é um $\mathfrak{g l}_{4}$-módulo simples, pelo Teorema 3.3.19, dado que $\mathcal{C}_{1}$ é o conjunto maximal de relações satisfeito por $T(v)$. A base de $V_{\mathcal{C}_{1}}(T(v))$ é:

$$
\mathcal{B}_{\mathcal{C}_{1}}(T(v))=L\left(\begin{array}{c}
\ell \leq m \leq r \leq 0 \\
-1 \leq s \leq 1 \\
t \leq 0 \\
t \leq n \leq s
\end{array}\right) .
$$

- Seja $\mathcal{C}_{2}$ o conjunto de relações admissível associado ao grafo 


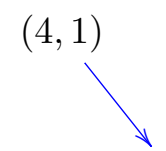

$(3,1)$

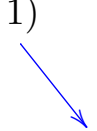

$(2,1)$
$(4,2)$

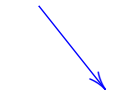

$(3,2)$

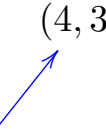
$(4,4)$

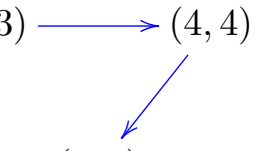

$(2,2)$

$(1,1)$

e $T\left(v+\delta^{11}\right)$ a tabela

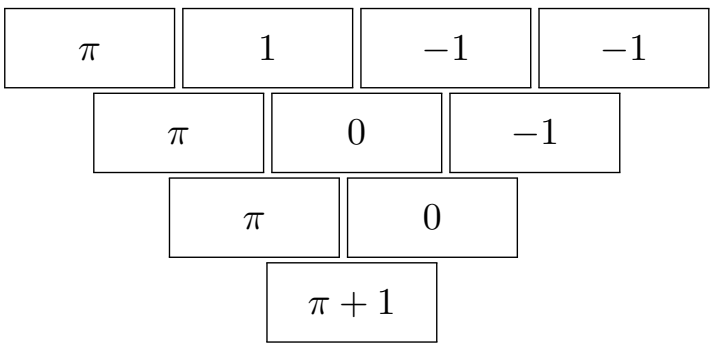

Assim, $V_{\mathcal{C}_{2}}\left(T\left(v+\delta^{11}\right)\right)$ é um $\mathfrak{g l}_{4}$-módulo simples, pelo Teorema 3.3.19, dado que $\mathcal{C}_{2}$ é o conjunto maximal de relações satisfeito por $T\left(v+\delta^{11}\right)$. A base de $V_{\mathcal{C}_{2}}(T(v))$ é:

$$
\mathcal{B}_{\mathcal{C}_{2}}(T(v))=L\left(\left(\begin{array}{c}
m \leq r \leq 0 \\
-1 \leq s \leq 1 \\
t \leq 0 \\
t \leq n \leq s \\
m \leq \ell
\end{array}\right)\right.
$$

- Seja $\mathcal{C}_{3}$ o conjunto de relações admissível associado ao grafo

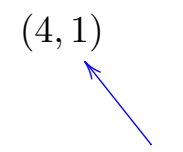

$(3,1)$
$(4,2)$

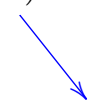

$(3,2)$

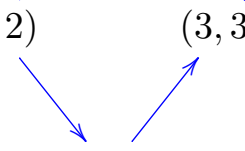

$(4,3)$

$(4,4)$

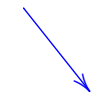

$(2,1)$

$(2,2)$

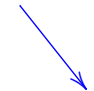

$(1,1)$

e $T\left(v+\delta^{31}\right)$ a tabela

\begin{tabular}{|l|l|l|l|l|}
\hline$\pi$ & & 1 & -1 & -1 \\
\hline$\pi+1$ & 0 & -1 \\
\hline
\end{tabular}




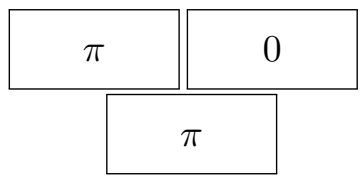

Desse modo, $V_{\mathcal{C}_{3}}\left(T\left(v+\delta^{31}\right)\right)$ é um $\mathfrak{g l}_{4}$-módulo simples, pelo Teorema 3.3.19, dado que $\mathcal{C}_{3}$ é o conjunto maximal de relações satisfeito por $T\left(v+\delta^{31}\right)$. A base de $V_{\mathcal{C}_{3}}(T(v))$ é:

$$
\mathcal{B}_{\mathcal{C}_{3}}(T(v))=L\left(\left(\begin{array}{c}
r \geq 0 \\
-1 \leq s \leq 1 \\
t \leq 0 \\
t \leq n \leq s \\
\ell \leq m \leq r
\end{array}\right) .\right.
$$

- Seja $\mathcal{C}_{4}$ o conjunto de relações admissível associado ao grafo

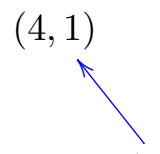

$(3,1)$

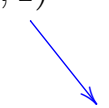

$(2,1)$
$(4,2)$

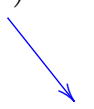

$(3,2)$

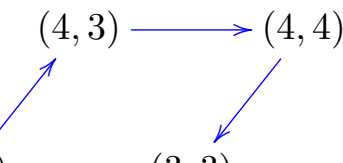

$(3,3)$

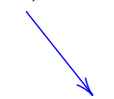

$(2,2)$

$(1,1)$

e $T\left(v+\delta^{11}+\delta^{31}\right)$ a tabela

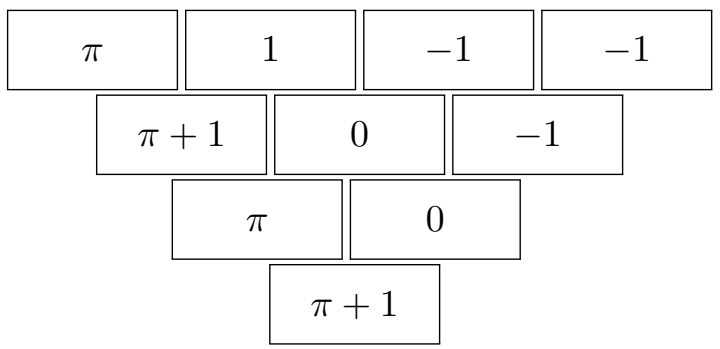

Dessa forma, $V_{\mathcal{C}_{4}}\left(T\left(v+\delta^{11}+\delta^{31}\right)\right)$ é um $\mathfrak{g l}_{4}$-módulo simples, pelo Teorema 3.3.19, dado que $\mathcal{C}_{4}$ é o conjunto maximal de relações satisfeito por $T\left(v+\delta^{11}+\delta^{31}\right)$. A base de $V_{\mathcal{C}_{4}}(T(v))$ é:

$$
\mathcal{B}_{\mathcal{C}_{4}}(T(v))=L\left(\left(\begin{array}{c}
r \geq 0 \\
-1 \leq s \leq 1 \\
t \leq 0 \\
t \leq n \leq s \\
m \leq \ell
\end{array}\right) .\right.
$$

Analisaremos a ação da subálgebra de Gelfand-Tsetlin $\Gamma$ no módulo $V_{\mathcal{C}}(T(v))$. 
Teorema 3.3.22 (Teorema 5.3 em [FRZ19]). Para qualquer conjunto admissível $\mathcal{C}$, o módulo $V_{\mathcal{C}}(T(v))$ é um módulo de Gelfand-Tsetlin com ação diagonalizável dos geradores da subálgebra de Gelfand-Tsetlin dados pela fórmula (3.2).

Teorema 3.3.23 (Teorema 5.8 em [FRZ19]). Para qualquer $\mathrm{m} \in \mathrm{Specm} \Gamma_{\text {st }}$ do suporte de GelfandTsetlin de $V_{\mathcal{C}}(T(v))$, a multiplicidade de Gelfand-Tsetlin de $\mathrm{m}$ é 1 .

A proposição a seguir fornece uma família de módulos de peso máximo que podem ser realizados como $V_{\mathcal{C}}(T(v))$ para algum conjunto admissível de relações $\mathcal{C}$.

Proposição 3.3.24 (Proposição 5.9 em [FRZ19]). Seja $\lambda=\left(\lambda_{1}, \ldots, \lambda_{n}\right)$. O módulo simples de peso máximo $L(\lambda)$ é um módulo de Gelfand-Tsetlin de $\Gamma_{\text {st }}$-relações se $\lambda_{i}-\lambda_{j} \notin \mathbb{Z}$ ou $\lambda_{i}-\lambda_{j}>i-j$ para qualquer $1 \leq i<j \leq n-1$.

\subsection{Módulos de Gelfand-Tsetlin para $\mathfrak{s l}_{n+1}$}

Nesta seção evidenciaremos o estudo dos módulos de Gelfand-Tsetlin para a álgebra $\mathfrak{s l}_{n+1}$. Por esse motivo, a partir deste momento, fixamos $\mathfrak{g}:=\mathfrak{s l}_{n+1}$, o conjunto de raízes simples $\Pi=$ $\left\{\alpha_{1}, \ldots, \alpha_{n}\right\}$ e definimos $\alpha_{r s}:=\alpha_{r}+\ldots+\alpha_{s}$ para $1 \leq r \leq s \leq n$. Usamos os elementos $E_{i j}$, $i, j=1, \ldots, n+1, i \neq j, H_{k}:=E_{k k}-E_{k+1, k+1}, k=1, \ldots, n$, como uma base de $\mathfrak{g}$.

Seja $\Gamma$ uma subálgebra de Gelfand-Tsetlin de $\mathfrak{g l}_{n+1}$. Consideramos a projeção natural

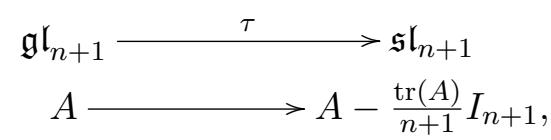

que se estende a um epimorfismo $\bar{\tau}: U\left(\mathfrak{g l}_{n+1}\right) \rightarrow U\left(\mathfrak{s l}_{n+1}\right)$. Então, a imagem $\bar{\tau}\left(\Gamma_{s t}\right)$ de $\Gamma_{s t}$ é chamada de subálgebra Gelfand-Tsetlin (padrão) $\mathbf{d e} \mathfrak{s l}_{n+1}$. Esta é uma subálgebra comutativa maximal de $U\left(\mathfrak{s l}_{n+1}\right)$ isomorfa a um anel de polinômios com $\frac{n(n+3)}{2}$ geradores. Recorrendo a um pequeno abuso de notação, denotamos a categoria de todos os $\mathfrak{s l}_{n+1}$-módulos de Gelfand-Tsetlin relativos a $\Gamma_{\text {st }}$ por $\mathcal{G} \mathcal{T}(n)$.

Observação 3.4.1. Como todas as bandeiras são conjugadas pelo grupo de Weyl, qualquer $\Gamma_{\mathcal{F}}$ é obtido a partir de $\Gamma_{s t}$ por conjugação. Escreveremos $\Gamma_{\mathcal{F}}=w \Gamma_{s t}$ para $w \in W$.

A partir deste momento assumiremos que $\mathcal{F}$ é a bandeira padrão e denotaremos $\Gamma_{s t}$ simplesmente por $\Gamma$. 
Definição 3.4.2. Seja $\mathcal{C}$ um conjunto de relações. Chamamos $\mathcal{C}$ admissivel se, para qualquer $\mathcal{C}$-realização $T(v), V_{\mathcal{C}}(T(v))$ tiver uma estrutura de um $\mathfrak{g}$-módulo, dotada da seguinte ação dos geradores de $\mathfrak{s l}_{n+1}$ :

$$
\begin{array}{r}
E_{k, k+1}(T(w))=-\sum_{i=1}^{k}\left(\frac{\prod_{j=1}^{k+1}\left(w_{k i}-w_{k+1, j}\right)}{\prod_{j \neq i}^{k}\left(w_{k i}-w_{k j}\right)}\right) T\left(w+\delta^{k i}\right), \\
E_{k+1, k}(T(w))=\sum_{i=1}^{k}\left(\frac{\prod_{j=1}^{k-1}\left(w_{k i}-w_{k-1, j}\right)}{\prod_{j \neq i}^{k}\left(w_{k i}-w_{k j}\right)}\right) T\left(w-\delta^{k i}\right), \\
H_{k}(T(w))=\left(2 \sum_{i=1}^{k} w_{k i}-\sum_{i=1}^{k-1} w_{k-1, i}-\sum_{i=1}^{k+1} w_{k+1, i}-1\right) T(w) .
\end{array}
$$

Neste caso diremos que $V_{\mathcal{C}}(T(v))$ é um $\mathfrak{g}$-módulo de Gelfand-Tsetlin de $\Gamma$-relações.

Exemplo 3.4.3. Como queremos trabalhar com $\mathfrak{s l}_{4}$, a seguir descrevemos explicitamente a ação da subálgebra de $\operatorname{Cartan}^{3}$ de $\mathfrak{s l}_{4}$ em qualquer $T(w) \in \mathcal{B}_{\mathcal{C}}(T(v))$.

- $H_{1}(T(w))=\lambda_{w}^{1} T(w)$, em que $\lambda_{w}^{1}=2 w_{11}-\left(w_{21}+w_{22}\right)-1$

- $H_{2}(T(w))=\lambda_{w}^{2} T(w)$, em que $\lambda_{w}^{2}=2\left(w_{21}+w_{22}\right)-\left(w_{31}+w_{32}+w_{33}\right)-w_{11}-1$

- $H_{3}(T(w))=\lambda_{w}^{3} T(w)$, em que $\lambda_{w}^{3}=2\left(w_{31}+w_{32}+w_{33}\right)-\left(w_{41}+w_{42}+w_{43}+w_{44}\right)-\left(w_{21}+w_{22}\right)-1$

Consequentemente, se $H_{1}(T(v))=\lambda_{v}^{1} T(v), H_{2}(T(v))=\lambda_{v}^{2} T(v)$ e $H_{3}(T(v))=\lambda_{v}^{3} T(v)$ para cada $(r, s, t, m, n, l) \in \mathbb{Z}^{6}$ e $T(w)=T(v+(r, s, t, m, n, l))=T\left(v+l \delta^{11}+m \delta^{21}+n \delta^{22}+r \delta^{31}+s \delta^{32}+t \delta^{31}\right)$, temos que:

- $\lambda_{w}^{1}=\lambda_{v}^{1}+2 l-(m+n)$

- $\lambda_{w}^{2}=\lambda_{v}^{2}+2(m+n)-(r+s+t+l)$

- $\lambda_{w}^{3}=\lambda_{v}^{3}+2(r+s+t)-(m+n)$

Exemplo 3.4.4. Se $\lambda$ for um peso de $\mathfrak{s l}_{4}$, de modo que $\left\langle\lambda+\rho, \alpha^{\vee}\right\rangle \notin \mathbb{Z}$ para qualquer $\alpha \in \Delta^{+}$, então o módulo de Verma $M(\lambda)$ admite uma realização por tabelas como um módulo fortemente genérico simples $V_{\mathcal{C}}(T(v))$, em que $v_{i}-v_{j} \notin \mathbb{Z}$, para cada $1 \leq i<j \leq 4, v_{1}+v_{2}+v_{3}+v_{4}=-6$, e $T(v)$ é a tabela genérica ${ }^{4}$

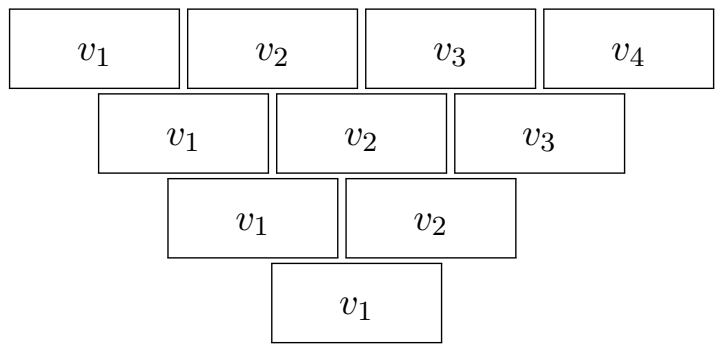

com conjunto de relações $\mathcal{C}$ definidas pelo seguinte grafo:

${ }^{3} h_{1}=E_{11}-E_{22}, h_{2}=E_{22}-E_{33}$ e $h_{3}=E_{33}-E_{44}$.

${ }^{4}$ Lembramos que a estrutura dos módulos genéricos foram estudados em [FGR15] 


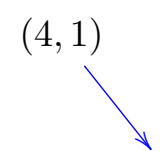

$(3,1)$
$(4,2)$

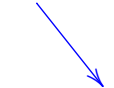

$(3,2)$

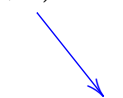

$(2,1)$

$(1,1)$

Uma base para este módulo é dada pelo conjunto de tabelas

$$
\mathcal{B}_{\mathcal{C}}(T(v))=L\left(\left\{\begin{array}{c}
l \leq m \leq r \leq 0 \\
n \leq s \leq 0
\end{array}\right) .\right.
$$

Isto é, $V_{\mathcal{C}}(T(v))=\operatorname{Span}_{\mathbb{C}} \mathcal{B}_{\mathcal{C}}(T(v))$, em que

$$
\mathcal{B}_{\mathcal{C}}(T(v))=\left\{T\left(v+l \delta^{11}+m \delta^{21}+n \delta^{22}+r \delta^{31}+s \delta^{32}+t \delta^{33}\right) \mid l \leq m \leq r \leq 0, n \leq s \leq 0, t \leq 0\right\} .
$$

A seguir descrevemos o reticulado de pesos com as correspondetes multiplicidades do módulo $V_{\mathcal{C}}(T(v))$. 


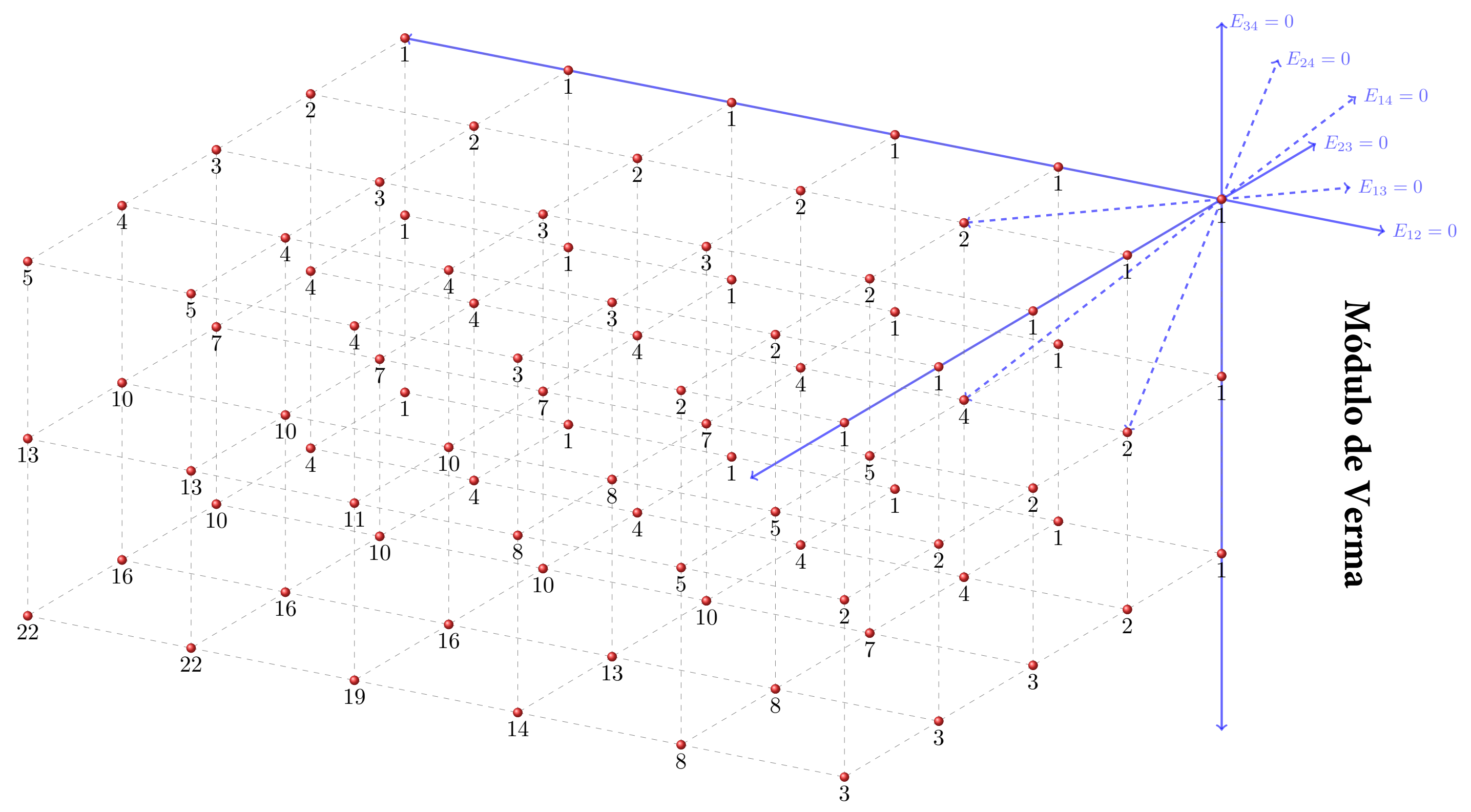


Uma construção semelhante pode ser feita para qualquer subálgebra de Gelfand-Tsetlin $\Gamma_{\mathcal{F}}$. Se $\Gamma_{\mathcal{F}}=w \Gamma_{s t}$ por algum $w \in W$, aplicando $w$ às fórmulas (3.3), obteremos uma ação explícita de $\mathfrak{s l}_{n+1}$ nos módulos de $\Gamma_{\mathcal{F}}$-relações de Gelfand-Tsetlin.

Observação 3.4.5. Observemos que cada módulo de peso com subespaços de peso de dimensão finita é um $\Gamma_{\mathcal{F}}$-módulo de Gelfand-Tsetlin, para qualquer $\Gamma_{\mathcal{F}}$, porém não necessariamente de relações.

\subsubsection{Classificação dos módulos de peso máximo de $\Gamma_{s t}$-relações}

O objetivo desta seção é descrever todos os g-módulos de peso máximo simples que podem ser realizados como $V_{\mathcal{C}}(T(v))$ para algum conjunto admissível de $\Gamma$-relações $\mathcal{C}$. Isto é, apresentamos um critério para determinar quais $\mathfrak{s l}_{n+1}$-módulos simples de peso máximo (limitados ou não) são módulos de $\Gamma$-relações. Em particular, obtemos um critério para determinar quais módulos de Verma simples e quais módulos de peso máximo limitados são módulos de $\Gamma$-relações.

Observação 3.4.6. A classificação de todos os $\mathfrak{s l}_{n+1}$-módulos de peso simples com espaços de peso de dimensão finita é conhecida (consulte detalhes em [Mat00]). Como a classificação de módulos de peso simples arbitrários está fora de alcance para $n \geq 2$, a classificação de $\mathfrak{s l}_{n+1}$-módulos simples de Gelfand-Tsetlin parece ser o próximo problema de classificação fundamental que se pode tentar resolver. Este problema foi resolvido para $\mathfrak{s l}_{3}$ em [FGR20]. Além disso, uma construção explícita de todos os $\mathfrak{s l}_{3}$-módulos de Gelfand-Tsetlin simples é fornecida em [FGR20].

Pela Proposição 3.3.24, temos a seguinte asserção.

Lema 3.4.7 (Lema 4.9 em [FHR20]). Se $\left\langle\lambda+\rho, \alpha^{\vee}\right\rangle \notin \mathbb{Z}_{\leq 0}$ para todo $\alpha \in \Delta^{+} \backslash\left\{\alpha_{k, n} \mid k=1, \ldots, n\right\}$, o módulo de peso máximo simples $L(\lambda)$ é um $\Gamma$-módulo de relações.

Corolário 3.4.8. Se $\lambda \in \mathfrak{h}^{*}$ for integral dominante ou dominante regular, então $L(\lambda)$ é um módulo de $\Gamma$-relações. Em particular, módulo simples de peso máximo admissível de nível $k$ é um módulo de relações.

Demonstração. Segue do Lema 3.4.7 e da definição de dominante regular ou integral dominante. Em particular, pelo Teorema 2.4.4 temos que se $L(\lambda)$ é admissível de nível $k$, então $\lambda$ é dominante regular.

A asserção a seguir classifica todos os módulos de peso máximo simples de $\Gamma$-relações. É importante observar que este é o primeiro resultado principal do nosso trabalho e será utilizado ao longo deste tese.

Teorema 3.4.9 (Teorema 4.11 em [FHR20]). O módulo de peso máximo simples $L(\lambda)$ é um módulo de $\Gamma$-relações se, e somente se, uma das seguintes condições for válida:

a) $\left\langle\lambda+\rho, \alpha^{\vee}\right\rangle \notin \mathbb{Z}_{\leq 0}$, para todo $\alpha \in \Delta^{+} \backslash\left\{\alpha_{k, n} \mid k=1, \ldots, n\right\}$. 
b) Existem $i, j$ únicos com $1 \leq i \leq j<n$, tais que:

i) $\left\langle\lambda+\rho, \alpha_{k}^{\vee}\right\rangle \in \mathbb{Z}_{>0}$ para cada $k>j$,

ii) $\left\langle\lambda+\rho, \alpha^{\vee}\right\rangle \notin \mathbb{Z}_{\leq 0}$ para todo $\alpha \in \Delta^{+} \backslash\left\{\alpha_{i, k} \mid k \geq j\right\}$,

iii) $\left\langle\lambda+\rho, \alpha_{i, n}^{\vee}\right\rangle \in \mathbb{Z}_{\leq 0}$.

Demonstração. Suponhamos que $L(\lambda)$ seja um módulo de relações. Então $L(\lambda) \simeq V_{\mathcal{C}}(T(v))$ para alguma tabela $T(v)$. Como $L(\lambda)$ é simples, podemos assumir sem perda de generalidade que $T(v)$ é o vetor de peso máximo $\lambda$ e $\mathcal{C}$ é o conjunto máximo de relações satisfeito por $T(v)$ (cf. Teorema 3.3.19). Então $E_{k, k+1}(T(v))=0$ para todo $k=1, \ldots, n$, o que implica $v_{i j}=v_{k j}$ para todo $1 \leq i, k<j \leq n+1(\operatorname{ver}(3.3))$ e $\mathcal{C} \supset\{((i+1, j) ;(i, j)) \mid 1 \leq j \leq i \leq n\}$. Defina $v_{j}:=v_{n+1, j}$, e observemos que $v_{i}-v_{j+1}=\left\langle\lambda+\rho, \alpha_{i, j}^{\vee}\right\rangle$ para todo $1 \leq i \leq j \leq n$. Agora suponhamos que para algum $\alpha \in \Delta^{+} \backslash\left\{\alpha_{k, n} \mid k=1, \ldots, n\right\}$ temos $\left\langle\lambda+\rho, \alpha^{\vee}\right\rangle \in \mathbb{Z}_{\leq 0}$. Portanto, existe um par $(r, s)$ com $1 \leq r \leq s<n$ tal que $v_{r}-v_{s+1}=\left\langle\lambda+\rho, \alpha_{r, s}^{\vee}\right\rangle \in \mathbb{Z}_{<0}$. Isso mostra que $I=\left\{(r, s) \mid v_{s}-v_{r} \in \mathbb{Z}_{>0}\right.$ e $1 \leq r<s<n\} \neq \emptyset$. Agora escolhamos $i=\min \{r \mid(r, s) \in I\}$ e $j=\min \{s \mid(i, s) \in I\}$. Então $(j, i)$ e $(j, j)$ formam um par adjunto em que o gráfico associado é:

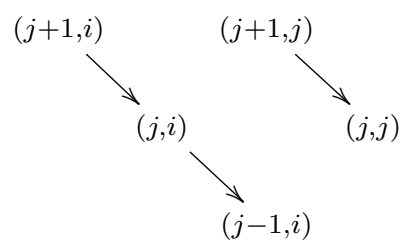

Por outro lado, $\mathcal{C}$ é um conjunto admissível de relações e, portanto, pela $\diamond$-condição o gráfico associado deve satisfazer

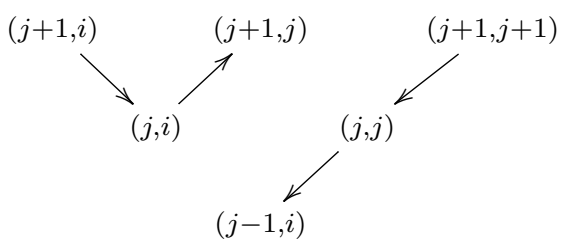

Portanto, $v_{j}-v_{j+1} \in \mathbb{Z}_{>0}$ e $v_{j+1}-v_{i} \in \mathbb{Z}_{>0}$. Repetindo o mesmo argumento, concluímos que $v_{k}-v_{k+1} \in \mathbb{Z}_{>0}$ para todos $k=j, \ldots, n$ e $v_{n+1}-v_{i} \in \mathbb{Z}_{\geq 0}$. Consequentemente, temos $v_{r}-v_{s} \in \mathbb{Z}_{>0}$ para todos os $j \leq r<s \leq n+1$. Por outro lado, da escolha de $i$ temos que $v_{r}-v_{s} \notin \mathbb{Z}_{\leq 0}$ para cada $1 \leq r<i$ e $s>r$. Da definição de $j$ também temos $v_{i}-v_{s} \notin \mathbb{Z}_{\leq 0}$ para cada $i<s<j$. Dado que $v_{s}-v_{i} \in \mathbb{Z}_{\geq 0}$ para todos $s \geq j$ e $v_{i}-v_{r} \notin \mathbb{Z}_{\leq 0}$ para todos $i<r<j$, obtemos $v_{s}-v_{r} \notin \mathbb{Z}_{\leq 0}$ para todos os $s, r$ de forma que $i<r<j \leq s \leq n+1$. Finalmente, $v_{s}-v_{r} \notin \mathbb{Z}_{\leq 0}$ para todo $s, r$ tal que $i<r<s<j$. De fato, suponhamos que $v_{s}-v_{r} \in \mathbb{Z}_{<0}$ para algum $i<r<s<j$. Então existe $r<j^{\prime} \leq s$ tal que $v_{k}-v_{k+1} \in \mathbb{Z}_{>0}$ para todo $k=j^{\prime}, \ldots, n$. Em particular, $v_{s}-v_{j} \in \mathbb{Z}_{>0}$, o que é uma contradição.

Por outro lado. Suponhamos primeiro que a) é válido, então pelo Lema 3.4.7 temos que $L(\lambda)$ é um módulo de $\Gamma$-relações. Agora assumamos b) e seja $v_{s}-v_{s+1}=\left\langle\lambda+\rho, \alpha_{s}^{\vee}\right\rangle$ para cada $1 \leq s \leq n$ tal que $\sum_{s=1}^{n+1} v_{s}=-\left(\begin{array}{c}n+1 \\ 2\end{array}\right)$. Então, para algum $1 \leq i \leq j<n$, as seguintes condições são satisfeitas:

- $v_{n+1}-v_{i} \in \mathbb{Z}_{\geq 0}$,

- $v_{r}-v_{s} \in \mathbb{Z}_{>0}$ para todo $j+1 \leq r<s \leq n+1$, 
- $v_{r}-v_{s} \notin \mathbb{Z}_{\leq 0}$ para todo $1 \leq r \leq j, r<s \leq n+1$ e $r, s \neq i$,

- $v_{r}-v_{i} \in \mathbb{Z}_{>0}$ para todo $j+1 \leq r \leq n$,

- $v_{r}-v_{i} \notin \mathbb{Z}_{\leq 0}$ para todo $1 \leq r \leq j$ e $r \neq i$.

Seja $T(v)$ uma tabela de Gelfand-Tsetlin com entradas

$$
v_{r s}= \begin{cases}v_{s}, & \text { se } 1 \leq s<i \text { ou } i \leq r \leq j, \\ v_{s+j-i+1}, & \text { se } i \leq s<r+i-j, \\ v_{s-r+j}, & \text { se } s \geq r+i-j,\end{cases}
$$

para $1 \leq s \leq r \leq n+1$, e seja $\mathcal{C}$ o conjunto máximo de relações satisfeitas por $T(v)$. Para provar que $\mathcal{C}$ é admissível, consideramos os seguintes casos.

Caso I: Suponhamos que $(k, r)$ e $(k, s)$ formem um par adjunto para algum $1 \leq k<j$. Então temos um subconjunto indecomponível $\mathcal{C}^{\prime}$ de $\mathcal{C}$ com o gráfico associado

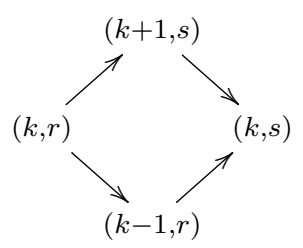

O mesmo acontece nos seguintes casos:

- $j \leq k \leq n$ e $1<s<i$;

- $j<k \leq n$ e $1 \leq r<s=i$;

- $j+1<k \leq n$ e $i<s<n+i-j$ em que $r=s-1$.

Caso II: Suponhamos que $j \leq k \leq n, 1 \leq r<i$ e $s \geq k+i-j$. Se $(k, r)$ e $(k, s)$ formam um par adjunto, então temos um subconjunto indecomponível $\mathcal{C}^{\prime}$ de $\mathcal{C}$ com o seguinte gráfico associado:

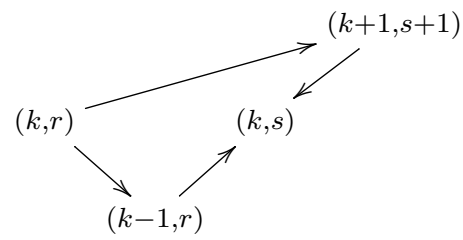

O gráfico é o mesmo no caso $k=j$ e $r \geq i$.

Caso III: Suponhamos que $j<k \leq n$ com $r \geq k+i-j$. Se $(k, r)$ e $(k, s)$ formam um par adjunto, então o gráfico associado do subconjunto indecomponível $\mathcal{C}^{\prime}$ de $\mathcal{C}$ é o seguinte:

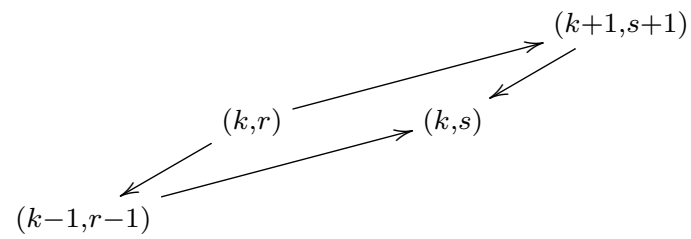


Caso IV: Finalmente, sejam $j<k \leq n, s=k+i-j$ e $r=s-1$. Neste caso $(k, r)$ e $(k, s)$ é um par adjunto com o gráfico associado da seguinte forma:

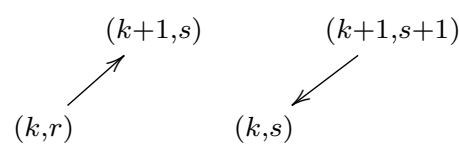

O mesmo acontece no caso: $j<k \leq n$ e $r=k+i-j-1$ para cada par adjunto $(k, r)$ e $(k, s)$.

Portanto $\mathcal{C}$ é admissível e $V_{\mathcal{C}}(T(v))$ é um módulo simples pelo Teorema 3.3.19. Portanto, $V_{\mathcal{C}}(T(v))=U(\mathfrak{g}) T(v), E_{k, k+1}(T(v))=0$ e $H_{k}(T(v))=\left\langle\lambda, \alpha_{k}^{\vee}\right\rangle T(v)$ para todos $k=1, \ldots, n$.

Em particular, aplicando o Teorema 3.4 .9 e a Proposição 1.4.13, obtemos o seguinte critério para que os módulos de peso máximo simples de dimensão infinita sejam módulos de $\Gamma$-relações com espaços de peso limitados.

Corolário 3.4.10 (Corolário 4.12 em [FHR20]). O módulo de peso máximo simples de dimensão infinita $L(\lambda)$ é um módulo de $\Gamma$-relações com espaços de peso limitados se, e somente se, uma das seguintes condições for válida:

a) $\left\langle\lambda+\rho, \alpha_{n}^{\vee}\right\rangle \notin \mathbb{Z}_{>0}$ e $\left\langle\lambda+\rho, \alpha_{k}^{\vee}\right\rangle \in \mathbb{Z}_{>0}$ para todo $k<n$;

b) $\left\langle\lambda+\rho, \alpha_{1}^{\vee}\right\rangle \notin \mathbb{Z} e\left\langle\lambda+\rho, \alpha_{k}^{\vee}\right\rangle \in \mathbb{Z}_{>0}$ para todo $k>1$;

c) $\left\langle\lambda+\rho, \alpha_{1}^{\vee}\right\rangle \in \mathbb{Z}_{<0} e\left\langle\lambda+\rho, \alpha_{1 n}^{\vee}\right\rangle \in \mathbb{Z}_{\leq 0} e\left\langle\lambda+\rho, \alpha_{k}^{\vee}\right\rangle \in \mathbb{Z}_{>0}$ para todo $k>1$;

d) Existe um único $i \in\{2, \ldots, n-1\}$, tal que $\left\langle\lambda+\rho, \alpha_{i}^{\vee}\right\rangle \in \mathbb{Z}_{<0},\left\langle\lambda+\rho, \alpha_{i-1, i}^{\vee}\right\rangle \in \mathbb{Z}_{>0},\left\langle\lambda+\rho, \alpha_{i n}^{\vee}\right\rangle \in$ $\mathbb{Z}_{\leq 0}$ e $\left\langle\lambda+\rho, \alpha_{k}^{\vee}\right\rangle \in \mathbb{Z}_{>0}$ para todo $k \neq i$

e) Existe um único $i \neq n$, tal que $\left\langle\lambda+\rho, \alpha_{i}^{\vee}\right\rangle \notin \mathbb{Z},\left\langle\lambda+\rho, \alpha_{i+1}^{\vee}\right\rangle \notin \mathbb{Z},\left\langle\lambda+\rho, \alpha_{i, i+1}^{\vee}\right\rangle \in \mathbb{Z}_{>0}$ e $\left\langle\lambda+\rho, \alpha_{k}^{\vee}\right\rangle \in \mathbb{Z}_{>0}$ para todos $k \neq i, i+1$.

Observação 3.4.11. Para $i=1,2, \ldots, n$ o módulo de peso máximo $L\left(-\omega_{i}\right)$ é limitado, mas não é um módulo de $\Gamma$-relações.

Corolário 3.4.12 (Corolário $4.14 \mathrm{em}$ [FHR20]). Seja $\lambda \in \mathfrak{h}^{*}$. As seguintes condições são equivalentes

a) O módulo de Verma $M(\lambda)$ é um módulo simples de $\Gamma$-relações;

b) $\left\langle\lambda+\rho, \alpha^{\vee}\right\rangle \notin \mathbb{Z}$ para qualquer $\alpha \in \Delta^{+} \backslash\left\{\alpha_{k, n} \mid k=1, \ldots, n\right\}$ e $\left\langle\lambda+\rho, \alpha_{k, n}^{\vee}\right\rangle \notin \mathbb{Z}_{>0}$ para $k=1, \ldots, n$.

Observação 3.4.13. Pelo Corolário 3.4.12, $M(\lambda)$ é um módulo de $\Gamma$-relações simples se, e somente se, $M(\lambda) \simeq V_{\mathcal{C}}(T(v))$, em que $T(v)$ é uma tabela genérica e $\mathcal{C}$ é o conjunto maximal de relações satisfeito por $T(v)$. Isso generaliza a Proposição 1 em [Maz98] (veja também [AFR17, Corolário 3.7] e [FRZ19, Exemplo 5.10]). A realização de todos os módulos de Verma simples é dada no Teorema $6.2 \mathrm{em}$ [FGRZ20].

A seguinte proposição generaliza o Corolário 3.4 .12

Corolário 3.4.14. Seja $\lambda \in \mathfrak{h}^{*}$ regular. As condições seguintes são equivalentes:

a) $O \mathfrak{s l}_{n+1}$-módulo de Verma generalizado $M_{\mathfrak{p}}^{\mathfrak{g}}(\lambda)$ é um $\Gamma$-módulo de relações simples; 
b) $\left\langle\lambda+\rho, \alpha^{\vee}\right\rangle \in \mathbb{Z}_{>0}$ para todo $\alpha \in \Delta_{\mathfrak{l}}^{+},\left\langle\lambda+\rho, \alpha^{\vee}\right\rangle \notin \mathbb{Z}$ para cada $\alpha \in \Delta_{\mathfrak{u}}^{+} \backslash\left\{\alpha_{k, n} \mid k=1, \ldots, n\right\}$, $e\left\langle\lambda+\rho, \alpha_{k, n}^{\vee}\right\rangle \notin \mathbb{Z}_{\geq 0}$ para qualquer $\alpha_{k, n} \in \Delta_{\mathfrak{u}}^{+}$tal que $k=1, \ldots, n$.

Demonstração. Segue do Teorema 1.3.9 e Teorema 3.4.9.

Pelo Teorema 3.4.9, temos os seguintes critérios para módulos $L(\lambda)$ com peso regular ou dominante serem módulos de relações.

Corolário 3.4.15. Seja $\lambda \in \mathfrak{h}^{*}$.

a) Suponhamos que $\lambda$ seja dominante. As seguintes condições são equivalentes:

(i) $L(\lambda)$ é um $\Gamma$-módulo de relações;

(ii) $\left\langle\lambda+\rho, \alpha^{\vee}\right\rangle \notin \mathbb{Z}_{\leq 0}$ para todo $\alpha \in \Delta^{+} \backslash\left\{\alpha_{k, n} \mid k=1, \ldots, n\right\}$ e $\left\langle\lambda+\rho, \alpha_{k, n}^{\vee}\right\rangle \notin \mathbb{Z}_{<0}$ para cada $k=1, \ldots, n$.

b) Suponhamos que $\lambda$ seja regular. Então $L(\lambda)$ é um $\Gamma$-módulo de relações se, e somente se, uma das seguintes condições for válida:

(i) $\left\langle\lambda+\rho, \alpha^{\vee}\right\rangle \notin \mathbb{Z}_{\leq 0}$ para todo $\alpha \in \Delta^{+} \backslash\left\{\alpha_{k, n} \mid k=1, \ldots, n\right\}$ e $\left\langle\lambda+\rho, \alpha_{k, n}^{\vee}\right\rangle \neq 0$ para cada $k=1, \ldots, n$;

(ii) Existem $i, j$ únicos com $1 \leq i \leq j<n$, tais que:

- $\left\langle\lambda+\rho, \alpha_{i n}^{\vee}\right\rangle \in \mathbb{Z}_{<0}$,

- $\left\langle\lambda+\rho, \alpha_{k}^{\vee}\right\rangle \in \mathbb{Z}_{>0}$ para cada $k=j+1, \ldots, n$,

- $\left\langle\lambda+\rho, \alpha^{\vee}\right\rangle \notin \mathbb{Z}_{\leq 0}$ para todo $\alpha \in \Delta^{+} \backslash\left\{\alpha_{i, k} \mid k=j, \ldots, n\right\}$.

A seguir apresentamos um critério que nos permite distinguir quais módulos de Verma simples projetivos na categoria $\mathcal{O}:=\mathcal{O}(\mathfrak{g})$ são módulos de relações. A definição e propriedades da categoria $\mathcal{O}$ podem ser encontradas na seç̧ão 1.1 em [Hum08].

Corolário 3.4.16. Suponhamos $\lambda \in \mathfrak{h}^{*}$. $O \mathfrak{s l}_{n+1}$-módulo $M(\lambda)$ é um módulo simples de $\Gamma$-relações projetivo em $\mathcal{O}$ se, e somente se, as duas condições a seguir forem satisfeitas:

a) $\left\langle\lambda+\rho, \alpha^{\vee}\right\rangle \notin \mathbb{Z}$ para todo $\alpha \in \Delta^{+} \backslash\left\{\alpha_{k n} \mid k=1, \ldots, n\right\}$;

b) $\left\langle\lambda+\rho, \alpha_{k n}^{\vee}\right\rangle \notin \mathbb{Z} \backslash\{0\}$ para cada $k=1, \ldots, n$.

Demonstração. Suponhamos que as condições a) e b) são verdadeiras, logo a condição b) no Corolário 3.4.12 é satisfeita, portanto $M(\lambda)$ é um módulo simples de $\Gamma$-relações. Por outro lado, $\lambda$ é dominante por definição, portanto pela proposição 3.8 em [Hum08] temos que $M(\lambda)$ é projetivo em $\mathcal{O}$. Reciprocamente, se $M(\lambda)$ é um módulo projetivo de $\Gamma$-relações simples, então a condição b) no Corolário 3.4.12 é verdadeira e $\left\langle\lambda+\rho, \alpha^{\vee}\right\rangle \notin \mathbb{Z}_{<0}$ para todo $\alpha \in\left\{\alpha_{k n} \mid k=1, \ldots, n\right\}$, uma vez que $\lambda$ é peso dominante (veja [Hum08]).

Como $L(\lambda)$ possui subespaços de peso de dimensão finita, então é um módulo de $\Gamma_{\mathcal{F}}$-GelfandTsetlin para qualquer bandeira $\mathcal{F}$. Denotamos por $\widetilde{W}$ a extensão do grupo de Weyl de $\mathfrak{g}$ pelas simetrias do sistema de raízes. Seja $w \in \widetilde{W}$ e $\mathfrak{b}=w \mathfrak{b}_{s t}$. Então $L_{\mathfrak{b}}(w \lambda)$ é um módulo de GelfandTsetlin de $\Gamma_{\mathcal{F}}$-relações se, e somente se, $w \lambda$ satisfizer as condições do Corolário 3.4.10 para $w \alpha_{k}^{\vee}$, $k=1, \ldots, n$. Em particular, obtemos 
Corolário 3.4.17 (Corolário 4.13 em [FHR20]). Seja $\lambda \in \mathfrak{h}^{*} e\left\langle\lambda+\rho, \beta_{j}^{\vee}\right\rangle \in \mathbb{Z}_{>0}$ para algumas raizes simples $\beta_{j}, j=1, \ldots, t$. Consideramos $w \in W$ de comprimento mínimo, tal que $\left\langle w(\lambda+\rho), \beta_{j}^{\vee}\right\rangle \in \mathbb{Z}_{\leq 0}$ para todo $j=1, \ldots, t$ e definamos $\Gamma_{\mathcal{F}}=w \Gamma$. Então o módulo de peso máximo simples $L(\lambda)$ é um módulo de Gelfand-Tsetlin de $\Gamma_{\mathcal{F}}$-relações limitado infinito-dimensional se, e somente se, $\lambda$ satisfazer uma das condições do Corolário 3.4.10.

O Corolário 3.4.17 mostra que $L(\lambda)$ pode ter realizações diferentes por tabelas de Gelfand-Tsetlin como um módulo de relações.

Lema 3.4.18 (Lema 4.14 em [FHR20]). Suponhamos que $\lambda$ satisfaz as condições a) ou e) do Corolário 3.4.10. Seja $i>1$ e $w_{i}=s_{i-1} s_{i} s_{i-2} s_{i-1} \cdots s_{2} s_{3} s_{1} s_{2} \in W$, então $L_{i}=L_{w_{i}^{-1} b_{s t}}\left(w_{i}^{-1} \lambda\right)$ é um módulo de $\Gamma$-relações.

Demonstração. Seja $\left\{v_{1}, \ldots, v_{n+1}\right\} \subseteq \mathbb{C}$ tal que $v_{1}-v_{2}=\left\langle\lambda+\rho, \alpha_{i}^{\vee}\right\rangle, v_{1}-v_{i+1}=-\left\langle\lambda+\rho, \alpha_{i-1}^{\vee}\right\rangle$, $v_{2}-v_{i+2}=\left\langle\lambda+\rho, \alpha_{i+1}^{\vee}\right\rangle, v_{k+2}-v_{k+3}=\left\langle\lambda+\rho, \alpha_{k}^{\vee}\right\rangle$ para todo $1 \leq k \leq i-2, v_{k}-v_{k+1}=\left\langle\lambda+\rho, \alpha_{k}^{\vee}\right\rangle$ para todo $k \geq i+2$, e $\sum_{i=1}^{n+1} v_{i}=-\left(\begin{array}{c}n+1 \\ 2\end{array}\right)$.

As entradas da tabela de Gelfand-Tsetlin $T(v)$ são:

$$
v_{r s}= \begin{cases}v_{1}+i-1, & \text { se } r=s=1, \\ v_{1}+i+1-r, & \text { se } 1<r \leq i \text { e } s=r-1, \\ v_{1}, & \text { se } r>i \text { e } s=i, \\ v_{2}+i+1-r, & \text { se } 1<r \leq i \text { e } s=r, \\ v_{2}, & \text { se } r>i \text { e } s=r, \\ v_{3}, & \text { se } r \geq 3 \text { e } s=1, \\ v_{s+2}, & \text { se } r \geq 4 \text { e } 2 \leq s<i, \\ v_{s}, & \text { se } r>s \geq i+1 .\end{cases}
$$

Definimos também o conjunto de relações $\mathcal{C}=\mathcal{C}^{+} \cup \mathcal{C}^{-}$, em que

$$
\begin{aligned}
\mathcal{C}^{+}:= & \{((r+1, s) ;(r, s)) \mid 1 \leq s<r \leq n \text { ou } r=s=1\} \bigcup\{((r+1, r+1) ;(r, r)) \mid i+1 \leq r \leq n\} \\
& \mathcal{C}^{-}:=\{((r, r) ;(r+1, r+1)) \mid 1<r \leq i\} \bigcup\{((r, s) ;(r+1, s+1)) \mid 1 \leq s<r \leq n\} .
\end{aligned}
$$

Neste caso, $\mathcal{C}$ é um conjunto de relações admissível, $T(V)$ uma $\mathcal{C}$-realização e $V_{\mathcal{C}}(T(v))$ um módulo simples, pelo Teorema 3.3.19. Mais ainda, $V_{\mathcal{C}}(T(v))=U(\mathfrak{g}) T(v), E_{12}(T(v))=E_{i+1,1}(T(v))=$ $E_{2, i+2}(T(v))=E_{k, k+1}(T(v))=0$ para todo $k \notin\{i-1, i, i+1\}, H_{1}(T(v))=\left\langle\lambda, \alpha_{i}^{\vee}\right\rangle T(v)$, $\sum_{k=1}^{i}\left(-H_{k}\right)(T(v))=\left\langle\lambda, \alpha_{i-1}^{\vee}\right\rangle T(v), \sum_{k=2}^{i+1} H_{k}(T(v))=\left\langle\lambda, \alpha_{i+1}^{\vee}\right\rangle T(v), H_{j+2}(T(v))=\left\langle\lambda, \alpha_{j}^{\vee}\right\rangle T(v)$ para todo $1 \leq j \leq i-2$ e $H_{j}(T(v))=\left\langle\lambda, \alpha_{j}^{\vee}\right\rangle T(v)$ para todo $j \geq i+2$. Portanto, $V_{\mathcal{C}}(T(v)) \simeq L_{i}$.

Observação 3.4.19. O módulo $L(-\rho)$ não é um módulo de $\Gamma$-relações, porém em [FGRZ20] pode ser encontrada uma realização por tabelas singulares críticas do módulo de Verma $M(-\rho)$. Além disso, em [SW20] foi descrita uma base para cada módulo de Verma. 


\subsection{Localização torcida de módulos de $\Gamma_{s t}$-relações}

Nesta seção aplicamos o funtor de localização torcida aos g-módulos de peso máximo que são de relações, o que nos permite estabelecer um critério para determinar quais $\mathfrak{g}$-módulos induzidos de $\mathfrak{s l}_{2}$ são módulos de relações para para $\mathfrak{g}=\mathfrak{s l}_{n+1}$. Nesta seção fixamos $\alpha=\alpha_{1}, f=f_{\alpha}$ e $\Gamma=\Gamma_{s t}$.

\subsubsection{Localização de módulos de $\Gamma$-relações}

Lema 3.5.1 (Lema 5.1 em [FHR20]). Suponhamos que $T(v)$ é uma $\mathcal{C}$-realização para algum conjunto admissivel de relações $\mathcal{C}$.

a) $f$ é injetivo em $V_{\mathcal{C}}(T(v))$ se, e somente se, $\mathcal{C} \cap\{((1,1) ;(2,1)),((1,1) ;(2,2))\}=\emptyset$.

b) $f$ é sobrejetiva em $V_{\mathcal{C}}(T(v))$ se, e somente se, $\mathcal{C} \cap\{((2,1) ;(1,1)),((2,2) ;(1,1))\}=\emptyset$.

Demonstração. Para qualquer tabela $T(w) \in \mathcal{B}_{\mathcal{C}}(T(v))$ temos que $f(T(w))=T\left(w-\delta^{11}\right) \in \mathcal{B}_{\mathcal{C}}(T(v))$, dado que $((1,1) ;(2,1)) \notin \mathcal{C}$ e $((1,1) ;(2,2)) \notin \mathcal{C}$. Suponhamos que $0 \neq u=\sum_{i \in I} a_{i} T\left(w^{i}\right) \in V_{\mathcal{C}}(T(v))$. Então

$$
f(u)=f\left(\sum_{i \in I} a_{i} T\left(w^{i}\right)\right)=\sum_{i \in I} a_{i} T\left(w^{i}-\delta^{11}\right) \neq 0,
$$

as $a_{i} \neq 0, T\left(w^{i}-\delta^{11}\right) \in V_{\mathcal{C}}(T(v))$ e $T\left(w^{i}-\delta^{11}\right) \neq T\left(w^{j}-\delta^{11}\right)$ para todo $i \neq j \in I$. Por outro lado, se $((1,1) ;(2,1)) \in \mathcal{C}$ ou $((1,1) ;(2,2)) \in \mathcal{C}$ então existe $T(w) \in \mathcal{B}_{\mathcal{C}}(T(v))$ tal que $w_{11}-w_{21}=1$ ou $w_{11}-w_{22}=1$, e portanto $f(T(w))=T\left(w-\delta^{11}\right)=0$, já que $T\left(w-\delta^{11}\right) \notin \mathcal{B}_{\mathcal{C}}(T(v))$. Isto mostra o item (a).

Se $((2,1) ;(1,1)) \notin \mathcal{C}$ e $((2,2) ;(1,1)) \notin \mathcal{C}$ então para qualquer tabela $T(w) \in \mathcal{B}_{\mathcal{C}}(T(v))$, temos que $T\left(w+\delta^{11}\right) \in \mathcal{B}_{\mathcal{C}}(T(v))$ e $f\left(T\left(w+\delta^{11}\right)\right)=T(w)$. Portanto, para qualquer $u \in V_{\mathcal{C}}(T(v))$,

$$
u=\sum_{i \in I} a_{i} T\left(w^{i}\right)=\sum_{i \in I} a_{i} f\left(T\left(w^{i}+\delta^{11}\right)\right)=f\left(\sum_{i \in I} a_{i} T\left(w^{i}+\delta^{11}\right)\right) .
$$

Por outro lado, assumamos que $((2,1) ;(1,1)) \in \mathcal{C}$ ou $((2,2) ;(1,1)) \in \mathcal{C}$ e $f$ é sobrejetivo em $V_{\mathcal{C}}(T(v))$. Então existe uma tabela $T(w) \in \mathcal{B}_{\mathcal{C}}(T(v))$ com $w_{11}=w_{21}$ ou $w_{11}=w_{22}$ e $u \in V_{\mathcal{C}}(T(v))$, tal que

$$
f(u)=\sum_{i \in I} a_{i} T\left(w^{i}-\delta^{11}\right)=T(w)
$$

Portanto, $T(w)=T\left(w^{i}-\delta^{11}\right)$ para algum $i \in I$. Como $T\left(w^{i}\right) \in \mathcal{B}_{\mathcal{C}}(T(v))$, temos que $0=w_{21}-w_{11}=$ $w_{21}^{i}-w_{11}^{i}+1 \in \mathbb{Z}_{>0}$ ou $0=w_{22}-w_{11}=w_{22}^{i}-w_{11}^{i}+1 \in \mathbb{Z}_{>0}$. Isto implica o item (b). 
A seguir consideramos a localização torcida de $V_{\mathcal{C}}(T(v))$ em relação a $f$ e sua realização por tabelas.

Lema 3.5.2 (Lema 5.2 em [FHR20]). Seja $\mathcal{C}$ um conjunto de relações admissível, tal que

$$
\mathcal{C} \cap\{((1,1) ;(2,1)),((1,1) ;(2,2))\}=\emptyset
$$

e $T(v)$ uma $\mathcal{C}$-realização. Se $\mathcal{D}=\mathcal{C} \backslash\{((2,1) ;(1,1)),((2,2) ;(1,1))\}$, então o módulo localizado $D_{f}\left(V_{\mathcal{C}}(T(v))\right)$ é isomorfo a $V_{\mathcal{D}}(T(v))$.

Demonstração. Seja $M=V_{\mathcal{C}}(T(v))$ e $N=V_{\mathcal{D}}(T(v))$. Pelo Lema 3.5.1, a ação de $f$ sobre $M$ é injetiva, mas não bijetiva. Portanto, $M$ é um submódulo próprio de $D_{f} M$. Por outro lado, consideremos o conjunto de relações $\mathcal{C}_{1}=\mathcal{D} \cup\{((1,1) ;(2,1))\}$. Pelo Teorema $3.3 .19, V_{\mathcal{C}_{1}}\left(T\left(v+\delta^{11}\right)\right)$ é um módulo simples isomorfo a $N / M$ e, portanto, $M$ é um submódulo maximal de $N$. Finalmente, como $f$ é bijetivo em $N$, pela Proposição 1.4 .22 temos $N \simeq D_{f} M$.

No teorema a seguir provamos que os funtores de localização $D_{f}$ e de torção $T_{\alpha}$ preservam a categoria de $\Gamma$-módulos de relações.

Teorema 3.5.3. Seja $M$ um $\Gamma$-módulo de relações com uma ação injetiva de $f$. Então $D_{f} M e$ $T_{\alpha} M$ são $\Gamma$-módulo de relações.

Demonstração. Segue dos Lemas 3.5.1 e 3.5.2.

Observação 3.5.4. Lembramos que usando (3.3), temos

$$
H_{k}(T(w))=\left(E_{k k}-E_{k+1, k+1}\right)(T(w))=\lambda_{k}^{w} T(w)
$$

em que

$$
\lambda_{k}^{w}:=2 \sum_{i=1}^{k} w_{k i}-\left(\sum_{i=1}^{k-1} w_{k-1, i}+\sum_{i=1}^{k+1} w_{k+1, i}+1\right)
$$

e consequentemente,

$$
\lambda_{k}^{w}=\lambda_{k}^{v}+2 \sum_{i=1}^{k} z_{k i}-\left(\sum_{i=1}^{k-1} z_{k-1, i}+\sum_{i=1}^{k+1} z_{k+1, i}\right),
$$

em que $T(w)=T(v+z)$ e $z \in \mathbb{Z}_{0}^{\frac{(n+1)(n+2)}{2}}$.

Antes de enunciar o próximo teorema, lembramos também que:

- $\left[E_{21}, E_{11}\right]=\left[E_{22}, E_{21}\right]=E_{21},\left[E_{12}, E_{21}\right]=h_{1},\left[E_{32}, E_{21}\right]=E_{31}$;

- $\left[E_{21}, E_{k, k+1}\right]=0$ para todo $k \geq 2$;

- $\left[E_{21}, E_{k k}\right]=\left[E_{21}, E_{k+1, k}\right]=0$ para todo $k \geq 3$.

Assim,

- $a d^{2}\left(E_{21}\right)\left(E_{11}\right)=a d^{2}\left(E_{21}\right)\left(E_{22}\right)=a d^{2}\left(E_{21}\right)\left(E_{32}\right)=0, a d^{3}\left(E_{21}\right)\left(E_{12}\right)=0$;

- $\operatorname{ad}\left(E_{21}\right)\left(E_{k, k+1}\right)=0$ para todo $k \geq 2$; 
- $\operatorname{ad}\left(E_{21}\right)\left(E_{k k}\right)=\operatorname{ad}\left(E_{21}\right)\left(E_{k+1, k}\right)=0$ para todo $k \geq 3$.

Portanto a ação dos geradores para $\mathfrak{s l}_{n+1}$ no módulo localizado torcido com relação a $E_{21}$ será dada por:

- $\Theta_{21}^{x}\left(E_{12}\right)=E_{12}-x h_{1} ; E_{21}^{-1}-x(x-1) E_{21}^{-1}$;

- $\Theta_{21}^{x}\left(E_{k, k+1}\right)=E_{k, k+1}$ para todo $k=2, \ldots, n$;

- $\Theta_{21}^{x}\left(E_{21}\right)=E_{21}$;

- $\Theta_{21}^{x}\left(E_{32}\right)=E_{32}-x E_{31} E_{21}^{-1}$;

- $\Theta_{21}^{x}\left(E_{k+1, k}\right)=E_{k+1, k}$ para todo $k=3, \ldots, n$;

- $\Theta_{21}^{x}\left(h_{1}\right)=h_{1}+2 x$

- $\Theta_{21}^{x}\left(h_{2}\right)=h_{2}-x$;

- $\Theta_{21}^{x}\left(h_{k}\right)=h_{k}$ para todo $k=3, \ldots, n$.

Sob as premissas do Lema 3.5.2, temos a seguinte asserção.

Lema 3.5.5 (Lema 5.3 em [FHR20]). Para qualquer tabela $T(w) \in \mathcal{B}_{\mathcal{C}}(T(v))$ e qualquer $x \in \mathbb{C}$ indicando por $T(w)^{x}$ a imagem de $T(w)$ como um elemento do módulo torcido $D_{f}^{x}\left(V_{\mathcal{C}}(T(v))\right)$. Temos: $E_{12} \cdot T(w)^{x}=-\left(w_{11}+x-w_{21}\right)\left(w_{11}+x-w_{22}\right) T\left(w+\delta^{11}\right)^{x}$.

$E_{k, k+1} \cdot T(w)^{x}=-\sum_{i=1}^{k}\left(\frac{\prod_{j=1}^{k+1}\left(w_{k i}-w_{k+1, j}\right)}{\prod_{j \neq i}^{k}\left(w_{k i}-w_{k j}\right)}\right) T\left(w+\delta^{k i}\right)^{x}$ para todo $k=2, \ldots, n$.

$E_{21} \cdot T(w)^{x}=T\left(w-\delta^{11}\right)^{x}$.

$E_{32} \cdot T(w)^{x}=\frac{w_{21}-\left(w_{11}+x\right)}{w_{21}-w_{22}} T\left(w-\delta^{21}\right)^{x}+\frac{w_{22}-\left(w_{11}+x\right)}{w_{22}-w_{21}} T\left(w-\delta^{22}\right)^{x}$.

$E_{k+1, k} \cdot T(w)^{x}=\sum_{i=1}^{k}\left(\frac{\prod_{j=1}^{k-1}\left(w_{k i}-w_{k-1, j}\right)}{\prod_{j \neq i}^{k}\left(w_{k i}-w_{k j}\right)}\right) T\left(w-\delta^{k i}\right)^{x}$ para todo $k=3, \ldots, n$.

$H_{1} \cdot T(w)^{x}=\left(\lambda_{1}^{w}+2 x\right) T(w)^{x}$.

$H_{2} \cdot T(w)^{x}=\left(\lambda_{2}^{w}-x\right) T(w)^{x}$.

$H_{k} \cdot T(w)^{x}=\lambda_{k}^{w} T(w)^{x}$ para todo $k=3, \ldots, n$.

Demonstração. Pelo Lema 3.5.2, temos que os elementos com base no módulo localizado são tabelas $T(w)$, em que $w_{11}=u_{1}+l$ para algum $l \in \mathbb{Z}$. Mais ainda, $E_{21}^{-1}(T(w))=T\left(w+\delta^{11}\right)$, por conseguinte temos:

$$
\begin{aligned}
E_{12} \cdot T(w)^{x} & =\left(\Theta_{21}^{x}\left(E_{12}\right)(T(w))\right)^{x} \\
& =\left(E_{12}(T(w))-x h_{1} E_{21}^{-1}(T(w))-x(x-1) E_{21}^{-1}(T(w))\right)^{x} \\
& =-\left(\left(w_{11}-w_{21}\right)\left(w_{11}-w_{22}\right)+x h_{1}+x(x-1)\right) T\left(w+\delta^{11}\right)^{x} \\
& =-\left(\left(w_{11}-w_{21}\right)\left(w_{11}-w_{22}\right)+x\left(\lambda_{w+\delta^{11}}^{1}+x-1\right)\right) T\left(w+\delta^{11}\right)^{x} \\
& =-\left(w_{11}+x-w_{21}\right)\left(w_{11}+x-w_{22}\right) T\left(w+\delta^{11}\right)^{x}
\end{aligned}
$$




$$
\begin{aligned}
& E_{k, k+1} \cdot T(w)^{x}=\left(\Theta_{21}^{x}\left(E_{k, k+1}\right)(T(w))\right)^{x}=\left(E_{k, k+1}(T(w))\right)^{x} \\
& =-\sum_{i=1}^{k}\left(\frac{\prod_{j=1}^{k+1}\left(w_{k i}-w_{k+1, j}\right)}{\prod_{j \neq i}^{k}\left(w_{k i}-w_{k j}\right)}\right) T\left(w+\delta^{k i}\right)^{x} \text {, for all } k=2, \ldots, n-1 \\
& E_{21} \cdot T(w)^{x}=\left(\Theta_{21}^{x}\left(E_{21}\right)(T(w))\right)^{x}=\left(E_{21}(T(w))\right)^{x}=T\left(w-\delta^{11}\right)^{x} \\
& E_{32} \cdot T(w)^{x}=\left(\Theta_{21}^{x}\left(E_{32}\right)(T(w))\right)^{x}=\left(E_{32}(T(w))-x E_{31} E_{21}^{-1}(T(w))\right)^{x} \\
& =\left(E_{32}(T(w))-x E_{31}\left(T\left(w+\delta^{11}\right)\right)\right)^{x} \\
& =\frac{w_{21}-w_{11}}{w_{21} \frac{w_{22}}{x}} T\left(w-\delta^{21}\right)^{x}+\frac{w_{22}-w_{11}}{w_{22}-w_{21}} T\left(w-\delta^{22}\right)^{x} \\
& -\frac{x}{w_{21}-w_{22}} T\left(w-\delta^{21}\right)^{x}-\frac{x}{w_{22}-w_{21}} T\left(w-\delta^{22}\right)^{x} \\
& =\frac{w_{21}-\left(w_{11}+x\right)}{w_{21}-w_{22}} T\left(w-\delta^{21}\right)^{x}+\frac{w_{22}-\left(w_{11}+x\right)}{w_{22}-w_{21}} T\left(w-\delta^{22}\right)^{x} \\
& E_{k+1, k} \cdot T(w)^{x}=\left(\Theta_{21}^{x}\left(E_{k+1, k}\right)(T(w))\right)^{x}=\left(E_{k+1, k}(T(w))\right)^{x} \\
& =\sum_{i=1}^{k}\left(\frac{\prod_{j=1}^{k-1}\left(w_{k i}-w_{k-1, j}\right)}{\prod_{j \neq i}^{k}\left(w_{k i}-w_{k j}\right)}\right) T\left(w-\delta^{k i}\right)^{x}, \text { for all } k=3, \ldots, n-1 \\
& H_{1} \cdot T(w)^{x}=\left(\Theta_{21}^{x}\left(h_{1}\right)(T(w))^{x}=\left(H_{1}(T(w))+2 x T(w)\right)^{x}=\left(\lambda_{1}^{w}+2 x\right) T(w)^{x}\right. \\
& H_{2} \cdot T(w)^{x}=\left(\Theta_{21}^{x}\left(h_{2}\right)(T(w))\right)^{x}=\left(H_{2}(T(w))-x T(w)\right)^{x}=\left(\lambda_{2}^{w}-x\right) T(w)^{x} \\
& H_{k} \cdot T(w)^{x}=\left(\Theta_{21}^{x}\left(h_{k}\right)(T(w))\right)^{x}=\left(H_{k}(T(w))\right)^{x}=\lambda_{k}^{w} T(w)^{x}, \text { for all } k=3, \ldots, n-1 .
\end{aligned}
$$

O seguinte resultado mostra que o funtor de localização torcida com respeito de $E_{21}$ preserva a categoria de módulos de $\Gamma$-relações, o qual generaliza a Proposição 8.7 em [FGR20], para $\mathfrak{g}=\mathfrak{s l}_{3}$ no caso genérico.

Teorema 3.5.6 (Teorema 5.4 em [FHR20]). Seja $M$ um $\Gamma$-módulo de relações com uma ação injetiva de $f$. Então $D_{f}^{x} M$ é um $\Gamma$-módulo de relações.

Demonstração. Por hipótese, $M \simeq V_{\mathcal{C}}(T(v))$ alguma $\mathcal{C}$-realização $T(v)$. Pelo Lema 3.5.2, o conjunto $\left\{T\left(v+\ell \delta^{11}\right) \mid \ell \in \mathbb{Z}\right\}$ é uma base do módulo localizado $D_{f} M \simeq V_{\mathcal{D}}(T(v))$. Agora definimos a transformação linear $\phi$ de $D_{f}^{x}\left(V_{\mathcal{C}}(T(v))\right)$ para $V_{\mathcal{D}}\left(T\left(v+x \delta^{11}\right)\right)$, tal que $\phi(T(w)):=T\left(w+x \delta^{11}\right)=$ $T\left(w^{x}\right) \in \mathcal{B}_{\mathcal{D}}\left(T\left(v+x \delta^{11}\right)\right)$ para cada tabela $T(w) \in \mathcal{B}_{\mathcal{C}}(T(v))$ e estenda por linearidade. Mais ainda, pelo Lema 3.5.5 temos:

$$
\begin{aligned}
\phi\left(E_{12} \cdot T(w)^{x}\right)= & -\left(w_{11}+x-w_{21}\right)\left(w_{11}+x-w_{22}\right) \phi\left(T\left(w+\delta^{11}\right)\right) \\
= & -\left(w_{11}+x-w_{21}\right)\left(w_{11}+x-w_{22}\right) T\left(w^{x}+\delta^{11}\right) \\
\phi\left(E_{k, k+1} \cdot T(w)^{x}\right)= & -\sum_{i=1}^{k}\left(\frac{\prod_{j=1}^{k+1}\left(w_{k i}-w_{k+1, j}\right)}{\prod_{j \neq i}^{k}\left(w_{k i}-w_{k j}\right)}\right) \phi\left(T\left(w+\delta^{k i}\right)\right) \\
= & -\sum_{i=1}^{k}\left(\frac{\prod_{j=1}^{k+1}\left(w_{k i}-w_{k+1, j}\right)}{\prod_{j \neq i}^{k}\left(w_{k i}-w_{k j}\right)}\right) T\left(w^{x}+\delta^{k i}\right), \text { for all } k=2, \ldots, n-1
\end{aligned}
$$




$$
\begin{aligned}
& \phi\left(E_{21} \cdot T(w)^{x}\right)=\phi\left(T\left(w-\delta^{11}\right)\right)=T\left(w^{x}+\delta^{11}\right) \\
& \phi\left(E_{32} \cdot T(w)^{x}\right)=\frac{w_{21}-\left(w_{11}+x\right)}{w_{21}-w_{22}} \phi\left(T\left(w-\delta^{21}\right)\right)+\frac{w_{22}-\left(w_{11}+x\right)}{w_{22}-w_{21}} \phi\left(T\left(w-\delta^{22}\right)\right) \\
& =\frac{w_{21}-\left(w_{11}+x\right)}{w_{21}-w_{22}} T\left(w^{x}-\delta^{21}\right)+\frac{w_{22}-\left(w_{11}+x\right)}{w_{22}-w_{21}} T\left(w^{x}-\delta^{22}\right) \\
& \phi\left(E_{k+1, k} \cdot T(w)^{x}\right)=\sum_{i=1}^{k}\left(\frac{\prod_{j=1}^{k-1}\left(w_{k i}-w_{k-1, j}\right)}{\prod_{j \neq i}^{k}\left(w_{k i}-w_{k j}\right)}\right) \phi\left(T\left(w-\delta^{k i}\right)\right) \\
& =\sum_{i=1}^{k}\left(\frac{\prod_{j=1}^{k-1}\left(w_{k i}-w_{k-1, j}\right)}{\prod_{j \neq i}^{k}\left(w_{k i}-w_{k j}\right)}\right) T\left(w^{x}-\delta^{k i}\right) \text {, for all } k=3, \ldots, n-1 \\
& \phi\left(H_{1} \cdot T(w)^{x}\right)=\left(\lambda_{1}^{w}+2 x\right) \phi(T(w))=\left(\lambda_{1}^{w}+2 x\right) T\left(w^{x}\right) \\
& \phi\left(H_{2} \cdot T(w)^{x}\right)=\left(\lambda_{2}^{w}-x\right) \phi(T(w))=\left(\lambda_{2}^{w}-x\right) T\left(w^{x}\right) \\
& \phi\left(H_{k} \cdot T(w)^{x}\right)=\lambda_{k}^{w} \phi(T(w))=\lambda_{k}^{w} T\left(w^{x}\right), \text { for all } k=3, \ldots, n-1
\end{aligned}
$$

Por outro lado, para cada tabela $T\left(w^{x}\right) \in \mathcal{B}_{\mathcal{C}^{\prime}}\left(T\left(v_{x}\right)\right)$, a ação de $\mathfrak{g}$ no módulo de relações $V_{\mathcal{C}^{\prime}}\left(T\left(v+x \delta^{11}\right)\right)$ dada pelas fórmulas de Gelfand-Tsetlin é:

$$
\begin{aligned}
& E_{12} \cdot \phi(T(w))=E_{12}\left(T\left(w^{x}\right)\right)=-\left(w_{11}+x-w_{21}\right)\left(w_{11}+x-w_{22}\right) T\left(w^{x}+\delta^{11}\right) \\
& E_{k, k+1} \cdot \phi(T(w))=E_{k, k+1}\left(T\left(w^{x}\right)\right) \\
& =-\sum_{i=1}^{k}\left(\frac{\prod_{j=1}^{k+1}\left(w_{k i}-w_{k+1, j}\right)}{\prod_{j \neq i}^{k}\left(w_{k i}-w_{k j}\right)}\right) T\left(w^{x}+\delta^{k i}\right), \text { for all } k=2, \ldots, n-1 \\
& E_{21} \cdot \phi(T(w))=E_{21}\left(T\left(w^{x}\right)\right)=T\left(w^{x}-\delta^{11}\right) \\
& E_{32} \cdot \phi(T(w))=E_{32}\left(T\left(w^{x}\right)\right)=\frac{w_{21}-\left(w_{11}+x\right)}{w_{21}-w_{22}} T\left(w^{x}-\delta^{21}\right)+\frac{w_{22}-\left(w_{11}+x\right)}{w_{22}-w_{21}} T\left(w^{x}-\delta^{22}\right) \\
& E_{k+1, k} \cdot \phi(T(w))=E_{k+1, k}\left(T\left(w^{x}\right)\right) \\
& =\sum_{i=1}^{k}\left(\frac{\prod_{j=1}^{k-1}\left(w_{k i}-w_{k-1, j}\right)}{\prod_{j \neq i}^{k}\left(w_{k i}-w_{k j}\right)}\right) T\left(w^{x}-\delta^{k i}\right), \text { for all } k=3, \ldots, n-1 \\
& H_{1} \cdot \phi(T(w))=H_{1}\left(T\left(w^{x}\right)\right)=\left(2\left(w_{11}+x\right)-\left(w_{21}+w_{22}\right)-1\right) T\left(w^{x}\right)=\left(\lambda_{1}^{w}+2 x\right) T\left(w^{x}\right) \\
& H_{2} \cdot \phi(T(w))=H_{2}\left(T\left(w^{x}\right)\right)=\left(2\left(w_{21}+w_{22}\right)-\left(w_{31}+w_{32}+w_{33}\right)-\left(w_{11}+x\right)-1\right) T\left(w^{x}\right) \\
& =\left(\lambda_{2}^{w}-x\right) T\left(w^{x}\right)
\end{aligned}
$$




$$
\begin{aligned}
H_{k} \cdot \phi(T(w)) & =H_{k}\left(T\left(w^{x}\right)\right) \\
& =\left(2 \sum_{i=1}^{k} w_{k i}-\left(\sum_{i=1}^{k-1} w_{k-1, i}+\sum_{i=1}^{k+1} w_{k+1, i}+1\right)\right) T\left(w^{x}\right) \\
& =\lambda_{k}^{w} T\left(w^{x}\right), \text { for all } k=3, \ldots, n-1 .
\end{aligned}
$$

Comparando a ação torcida de $\mathfrak{g}$ do Lema 3.5.5 com as fórmulas de Gelfand-Tsetlin, temos

$$
\phi\left(g \cdot T(w)^{x}\right)=g \phi\left(T(w)^{x}\right)
$$

para qualquer $g \in \mathfrak{g}$. Portanto, $\phi$ é um isomorfismo de módulos, o qual completa a prova.

Corolário 3.5.7 (Corolário 5.4 em [FHR20]). Seja M um módulo simples de $\Gamma$-relações.

a) Se $f$ é bijetivo em $M, M$ é isomorfo com $D_{f}^{x}(N)$ para algum módulo de $\Gamma$-relações simples $N$ com uma ação injetora de $f$ e $x \in \mathbb{C} \backslash \mathbb{Z}$.

b) Se $f$ é sobrejetivo em $M$, mas não injetivo, então $M$ é isomorfo com $T_{\alpha}(N)=\frac{D_{f}(N)}{N}$ para algum módulo de $\Gamma$-relações simples $N$ com um ação injetiva de $f$.

Demonstração. Como $M$ é um módulo simples de $\Gamma$-relações, então $M \simeq V_{\mathcal{C}}(T(v))$ para alguma tabela de Gelfand-Tsetlin e um conjunto máximo admissível de relações $\mathcal{C}$ satisfeito por $T(v)$.

Parte (a): Suponhamos que $f$ seja bijetivo em $M$. Pelo Lema 3.5.1, temos $v_{11}-v_{21} \notin \mathbb{Z}$ e $v_{11}-v_{22} \notin \mathbb{Z}$. Sem perda de generalidade, assumimos que $v_{21}-v_{22} \notin \mathbb{Z}$. Neste caso, seja $x=v_{11}-v_{21}$ e consideremos a tabela de Gelfand-Tsetlin $T\left(v^{\prime}\right)=T\left(v+x \delta^{11}\right)$. Então $\mathcal{C}^{\prime}=\mathcal{C} \cup\{((2,1) ;(1,1))\}$ é o conjunto máximo admissível de relações satisfeitas por $T\left(v^{\prime}\right)$. Portanto, pelo Teorema 3.3.19, $N=V_{\mathcal{C}^{\prime}}\left(T\left(v^{\prime}\right)\right)$ é um módulo de relações simples. Pelo Lema 3.5.1, $f$ age injetivamente em $N$, mas não sobrejetivamente. Então $D_{f}^{x} N \simeq M$ pelo Teorema 3.5.6.

Parte (b): Suponhamos que $f$ seja sobrejetiva em $M$, mas não injetiva. Pelo Lema 3.5.1, podemos assumir que $v_{11}-v_{21} \in \mathbb{Z}_{>0}$ e $v_{11}-v_{22} \notin \mathbb{Z}$. Definamos $x=v_{11}-v_{21}$ e $T\left(v^{\prime}\right)=T\left(v+x \delta^{11}\right)$. Então $\left.\mathcal{C}^{\prime}=(\mathcal{C} \backslash\{((1,1) ;(2,1))\}) \cup\{((2,1)) ;(1,1))\right\}$ é o conjunto máximo admissível de relações satisfeitas por $T\left(v^{\prime}\right)$. Portanto, $N=V_{\mathcal{C}^{\prime}}\left(T\left(v^{\prime}\right)\right)$ é um módulo de relações simples pelo Teorema 3.3.19. Do Lema 3.5.1 temos que $f$ age injetivamente em $N$, e que o módulo localizado $D_{f}(N)$ é isomorfo a $V_{\mathcal{C} \backslash\{((1,1) ;(2,1))\}}\left(T\left(v^{\prime}\right)\right)$ (cf. Lema 3.5.2). Além disso, $N$ é um submódulo máximaal de $D_{f}(N)$ e, portanto, $D_{f}(N) / N \simeq V_{\mathcal{C}}\left(T\left(v^{\prime}\right)\right)$ é um módulo simples. Concluímos que $D_{f}(N) / N \simeq V_{\mathcal{C}}(T(v))$.

\subsection{2 $\mathfrak{s l}_{2}$-módulos induzidos de $\Gamma_{\alpha}$-relações}

Seja $\mathfrak{p} \subset \mathfrak{g}$ uma subálgebra parabólica de $\mathfrak{g}$ com o fator Levi isomorfo para $\mathfrak{a}_{\alpha}=\mathfrak{s l}_{2}+\mathfrak{h}$ com base na raiz $\alpha:=\alpha_{1}$. Seja $V=V(\gamma, \mu)$ um $\mathfrak{a}_{\alpha}$-módulo de peso simples cuspidal, em que $\mu \in \mathfrak{h}^{*}$ é tal que $V_{\mu} \neq 0$ e $\gamma \in \mathbb{C}$ é o autovalor do elemento Casimir $c_{\alpha}=\left(H_{\alpha}+1\right)^{2}+4 F_{\alpha} E_{\alpha}$ de $\mathfrak{a}_{\alpha}$. Denotamos 
por $L(\gamma, \mu)=L_{\mathfrak{p}}^{\mathfrak{g}}(\{\alpha\}, V)$ o único quociente simples do módulo induzido $\operatorname{Ind}_{\mathfrak{p}}^{\mathfrak{g}}(\{\alpha\}, V)$ em que a ação do radical de $\mathfrak{p}$ em $V$ é trivial. Seja $\Gamma_{\alpha}$ qualquer subálgebra de Gelfand-Tsetlin correspondente à bandeira que contém $\mathfrak{a}_{\alpha}$.

A seguir provaremos que todo $\mathfrak{s l}_{2}$-módulo simples cuspidal é um módulo de relações, mais especificamente é um módulo genérico (veja Teorema 4.1.2 e exercício 4.1.5 em [Maz10]). Também calculamos o anulador dele (veja 4.9.22. em [Dix77]).

Proposição 3.5.8. Seja $\mathfrak{a}=\mathfrak{s l}_{2}$ com a base padrão $\{e, f, h\}, a, b \in \mathbb{C}$ e $V=V(a, b)$ um a-módulo de peso denso simples, em que a é um autovalor de $h$ e $b$ é um autovalor do elemento Casimir $c_{\alpha}$ de a $\mathfrak{a}$ Então $V$ é um módulo de $\Gamma$-relações. Além disso, seja $x \in \mathbb{C}$ uma raiz do polinômio $(2 x-a-1)^{2}-b$ tal que $\lambda:=a-2 x \notin \mathbb{Z}_{\geq 0}$, então o a-módulo de Verma $M(\lambda)$ é um módulo de $\Gamma_{\text {st }}$-relações simples e $\operatorname{Ann}_{U(\mathfrak{a})} V=\operatorname{Ann}_{U(\mathfrak{a})} M(\lambda)=U(\mathfrak{a})\left(c_{\alpha}-\lambda\right)$.

Demonstração. Dado que $V$ é um a-módulo simples, então $b \neq(a-2 k+1)^{2}$ para todo $k \in \mathbb{Z}$ (veja [Maz10]), portanto $x \notin \mathbb{Z}$. Por outro lado, $\lambda$ é um peso integral se, e somente se, $b=m^{2}$ por algum $m \in \mathbb{Z}_{\geq 0}$.

Caso não integral: Neste caso, $\lambda$ não é integral, mas é dominante regular e antidominante.

Seja $T(v)=T\left(v_{i j}\right)$ com $1 \leq j \leq i \leq 2$ a tabela de Gelfand-Tsetlin, de modo que $v_{11}=v_{21}$, $v_{22}=-v_{21}-1$ e $v_{21}=\frac{1}{2} a x$, então $T(v)$ satisfaz o conjunto de relações $\mathcal{C}=\{((2,1) ;(1,1))\}$. Além disso, $\mathcal{C}$ é o conjunto de relações maximal que $T(v)$ satisfaz, portanto $V_{\mathcal{C}}(T(v))$ é um módulo de relações simples (veja Teorema 3.3.19). Como $E_{12}(T(v))=0$ e $h(T(v))=\lambda T(v)$, temos que $V_{\mathcal{C}}(T(v)) \simeq L(\lambda)=M(\lambda)$, pois $\lambda$ é antidominante. Por outro lado, $c_{\alpha}(T(v))=(\lambda+1)^{2} T(v)=b T(v)$.

Agora, consideramos a tabela $T(u)=T\left(v+x \delta^{11}\right)$, em que $u_{11}=v_{21}+x, u_{21}=v_{21}$ e $u_{22}=v_{22}$. Logo $u_{11}-u_{21} \notin \mathbb{Z}, u_{11}-u_{22} \notin \mathbb{Z}$ e $u_{21}-u_{22} \notin \mathbb{Z}$, então $V_{\emptyset}(T(u))$ é um módulo de relações simples (veja Teorema 3.3.19). Observamos que $E_{21}$ e $E_{12}$ agem injetivamente em $V_{\emptyset}\left(T(u)\right.$ ), portanto $V_{\emptyset}(T(u)$ ) é um módulo denso (veja Proposição 1.4.3). Além disso, $h(T(u))=a T(u)$ e $c_{\alpha}(T(u))=b T(u)$, portanto $V_{\emptyset}(T(u)) \simeq V$ e do Teorema 3.5.6, temos que $V_{\emptyset}(T(u)) \simeq D_{f}^{x} L(\lambda)$. Por conseguinte, $\operatorname{Ann}_{U(\mathfrak{a})} V=\operatorname{Ann}_{U(\mathfrak{a})} D_{f}^{x} L(\lambda)=\operatorname{Ann}_{U(\mathfrak{a})} L(\lambda)=U(\mathfrak{a})\left(c_{\alpha}-\lambda\right)$, do Teorema 1.4.27 e do Teorema 1.3.14.

Caso integral: Neste caso $\lambda=-m-1$ em que $m \in \mathbb{Z}_{\geq 0}$.

Seja $T(v)=T\left(v_{i j}\right)$ com $1 \leq j \leq i \leq 2$ a tabela de Gelfand-Tsetlin, de modo que $v_{11}=v_{22}=$ $-\frac{1}{2}(m+1)$ e $v_{21}=\frac{1}{2}(m-1)$, então $E_{12}(T(v))=0, h(T(v))=\lambda T(v)$ e $c_{\alpha}(T(v))=b T(v)$, portanto $V_{\mathcal{C}}(T(v)) \simeq L(\lambda)=M(\lambda)$, em que $\mathcal{C}=\{((2,1) ;(2,2)),((2,2) ;(1,1))\}$ é o conjunto de relações maximal satisfeito por $T(v)$.

No Teorema 3.5.6, temos que $D_{f}^{x} L(\lambda) \simeq V_{\mathcal{D}}(T(u))$, em que $T(u)=T\left(u_{i j}\right)$ é a tabela de GelfandTsetlin com altura 2 , de modo que $u_{11}=\frac{1}{2} a, u_{21}=v_{21}, u_{22}=v_{22}$ e $\mathcal{D}=\{((2,1) ;(2,2))\}$ é o conjunto de relações maximal que $T(u)$ satisfaz, dado que $u_{11}-u_{21} \notin \mathbb{Z}, u_{11}-u_{22} \notin \mathbb{Z}$ e $u_{21}-u_{22} \in \mathbb{Z}_{\geq 0}$. Como $E_{21}$ e $E_{12}$ agem injetivamente em $V_{\mathcal{D}}(T(u))$, temos que $V_{\mathcal{D}}(T(u))$ é um módulo denso (veja Proposição 1.4.3) e dado que $h(T(u))=a T(u)$ e $c_{\alpha}(T(u))=b T(u)$, concluímos que $V_{\mathcal{D}}(T(u)) \simeq V$. Assim, $\operatorname{Ann}_{U(\mathfrak{a})} V=\operatorname{Ann}_{U(\mathfrak{a})} D_{f}^{x} L(\lambda) \supset A n n_{U(\mathfrak{a})} L(\lambda)$ pelo Lema 1.4.24. No entanto, o anulador de $L(\lambda)$ é maximal (veja 4.9.22. em [Dix77]). Isso prova que $\operatorname{Ann}_{U(\mathfrak{a})} V=\operatorname{Ann}_{U(\mathfrak{a})} M(\lambda)$.

Teorema 3.5.9 (Teorema 5.6 em [FHR20]). Seja $n>1$. O módulo $L(\gamma, \mu)$ é um módulo de GelfandTsetlin de $\Gamma_{\text {st }}$-relações se, e somente se, $L(\lambda)$ for um $\mathfrak{g}$-módulo de peso máximo de $\Gamma_{\text {st }}$-relações, em 
que

$$
\left\{\begin{array}{l}
\left\langle\mu-\lambda, \alpha_{1}^{\vee}\right\rangle=2 x ; \\
\left\langle\mu-\lambda, \alpha_{2}^{\vee}\right\rangle=-x ; \\
\left\langle\mu-\lambda, \alpha_{i}^{\vee}\right\rangle=0 \text { para cada } i=3, \ldots, n ; \\
\left\langle\lambda+\rho, \alpha_{1}^{\vee}\right\rangle \notin \mathbb{Z}_{\geq 0} ; \\
\left(2 x-\mu_{1}-1\right)^{2}=\gamma .
\end{array}\right.
$$

Neste caso, $L(\gamma, \mu) \simeq D_{f}^{x}(L(\lambda))$ e $x$ satisfaz à condição $x-\left\langle\mu+\rho, \alpha_{1}^{\vee}\right\rangle \notin \mathbb{Z}$. Além disso,

a) $L(\gamma, \mu)$ é limitado se, e somente se, $L(\lambda)$ for limitado;

b) Se $\lambda$ for dominante, $s_{1} \cdot \lambda$ será dominante e $\gamma \neq m^{2}$ para todo $m \in \mathbb{Z}$. Neste caso, $A_{n n_{U(\mathfrak{g})}} L(\gamma, \mu)=$ $A n n_{U(\mathfrak{g})} L(\lambda)=A n n_{U(\mathfrak{g})} L\left(s_{1} \cdot \lambda\right) ;$

c) Se $\gamma=m^{2}$ por algum $m \in \mathbb{Z} \backslash\{0\}$, então $L(\lambda)$ é limitado e o peso $w \cdot \lambda$ é integral para todo $w \in W$. Neste caso, $A n n_{U(\mathfrak{g})} L(\lambda) \subset A n n_{U(\mathfrak{g})} L(\gamma, \mu) \subset A n n_{U(\mathfrak{g})} L(w \cdot \lambda)$, se $w \cdot \lambda$ for dominante.

Demonstração. Como $V$ é um $\mathfrak{s l}_{2}$-módulo simples e denso, então $\gamma \neq\left(\mu_{1}-2 k+1\right)^{2}$ para todo $k \in \mathbb{Z}$, e portanto $x \notin \mathbb{Z}$. Por outro lado, $\gamma=m^{2}$ para algum $m \in \mathbb{Z}$ se, e somente se, $\left\langle\lambda+\rho, \alpha_{1}^{\vee}\right\rangle \in \mathbb{Z}$. Consideremos os dois casos a seguir:

Caso 1: Seja $\gamma \neq m^{2}$ para todo $m \in \mathbb{Z}$. Suponhamos que $L(\gamma, \mu)$ é um $\mathfrak{g}$-módulo de GelfandTsetlin de $\Gamma_{\alpha}$-relações. Então $L(\gamma, \mu) \simeq V_{\mathcal{C}}(T(v))$ para algum conjunto de relações $\mathcal{C}$ e uma tabela $T(v)=T\left(v_{i j}\right)$, de modo que

$$
v_{i j}= \begin{cases}v_{1}+x, & \text { se } i=1 \\ v_{1}, & \text { se } i>j=1 \\ v_{j}, & \text { se } j \geq 2,\end{cases}
$$

com

- $v_{1}-v_{2}=\left\langle\mu+\rho, \alpha_{1}^{\vee}\right\rangle-2 x ;$

- $v_{2}-v_{3}=\left\langle\mu+\rho, \alpha_{2}^{\vee}\right\rangle+x$

- $v_{j}-v_{j+1}=\left\langle\mu+\rho, \alpha_{j}^{\vee}\right\rangle$ para cada $3 \leq j \leq n$;

- $\sum_{j=1}^{n+1} v_{j}=-n-1$.

Por outro lado, $f$ é bijetivo em $V_{\mathcal{C}}(T(v))$ se, e somente se, $x-\left\langle\mu, \alpha_{1}^{\vee}\right\rangle \notin \mathbb{Z}$, neste caso

$$
\mathcal{C} \cap\{((1,1) ;(2,1)),((1,1) ;(2,2)),((2,1) ;(1,1)),((2,2) ;(1,1))\}=\emptyset .
$$

Consideremosa tabela $T\left(v^{\prime}\right)=T\left(v_{i j}^{\prime}\right)$ com entradas $v_{11}^{\prime}=v_{21}$ e $v_{i j}^{\prime}=v_{i j}$ para cada $i \neq 1$. Então $\mathcal{D}=$ $\mathcal{C} \cup\{((2,1) ;(1,1))\}$ é o conjunto máximo de relações satisfeitas por $T\left(v^{\prime}\right)$, uma vez que $\left\langle\lambda+\rho, \alpha_{1}^{\vee}\right\rangle \notin \mathbb{Z}$. Portanto, o módulo $V_{\mathcal{D}}\left(T\left(v^{\prime}\right)\right)$ é simples pelo Teorema 3.3.19. Então $V_{\mathcal{D}}\left(T\left(v^{\prime}\right)\right) \simeq U(\mathfrak{g}) T\left(v^{\prime}\right)$, $E_{k, k+1}\left(T\left(v^{\prime}\right)\right)=0$ e $H_{k}(T(v))=\lambda_{k} T(v)$ para todo $k=1, \ldots, n, \lambda_{k}=\left\langle\lambda, \alpha_{k}^{\vee}\right\rangle$. Consequentemente 
temos que $V_{\mathcal{D}}\left(T\left(v^{\prime}\right)\right) \simeq L(\lambda)$. Além disso, $c_{\alpha}\left(T\left(v^{\prime}\right)\right)=\left(\lambda_{1}+1\right)^{2} T\left(v^{\prime}\right)=\gamma T\left(v^{\prime}\right)$. Aplicando o Teorema 3.5.6, concluímos que $D_{f}^{x} L(\lambda) \simeq L(\gamma, \mu)$.

Reciprocamente, seja $L(\lambda)$ um módulo de Gelfand-Tsetlin $\Gamma_{\alpha}$-relações. Então $L(\lambda) \simeq V_{\mathcal{C}}(T(v))$, em que $T(v)=T\left(v_{i j}\right)$ é uma tabela de Gelfand-Tsetlin tal que $v_{i j}=v_{j} \operatorname{com} v_{j}-v_{j+1}=\left\langle\lambda+\rho, \alpha_{j}^{\vee}\right\rangle$

para cada $1 \leq j \leq n$, e $\sum_{j=1}^{n+1} v_{j}=-n-1$. Observemos que $\mathcal{C}$ é o conjunto máximo de relações satisfeitas por $T(v)$. Sem perda de generalidade, assumimos que $((2,1) ;(1,1)) \in \mathcal{C}$ e $((1,1) ;(2,1)) \notin \mathcal{C}$. Portanto, o módulo localizado $D_{f}^{x}(L(\lambda))$ é isomorfo a $V_{\mathcal{D}}\left(T\left(v+x \delta^{11}\right)\right)$, em que $\mathcal{D}=\mathcal{C} \backslash\{((2,1) ;(1,1))\}$ pelo Teorema 3.5.6. Dado que $x+v_{11}-v_{22} \notin \mathbb{Z}$, então $V_{\mathcal{D}}\left(T\left(v+x \delta^{11}\right)\right)$ é um módulo simples. Além disso, o $\mathfrak{s l}_{2}$-módulo $\operatorname{span}_{\mathbb{C}}\left\{T\left(v+(x+\ell) \delta^{11}\right) \mid \ell \in \mathbb{Z}\right\}$ é isomorfo a $V$. De (3.3), temos $E_{k, k+1}\left(T\left(v+(x+\ell) \delta^{11}\right)\right)=0$ para todo $k=2, \ldots, n$ e $\ell \in \mathbb{Z}$. Portanto, temos um epimorfismo de $U(\mathfrak{g})$-módulos

$$
\phi: M_{\mathfrak{p}}^{\mathfrak{g}}(\{\alpha\}, V) \rightarrow V_{\mathcal{D}}\left(T\left(v+x \delta^{11}\right)\right),
$$

de modo que $\phi(u \otimes T(v))=u T(v)$ para todo $u \in U(\mathfrak{g})$. Portanto, $L(\gamma, \mu) \simeq V_{\mathcal{D}}\left(T\left(v+x \delta^{11}\right)\right)$.

Caso 2: Seja $\gamma=m^{2}$ para algum $m \in \mathbb{Z}$. Neste caso $\left\langle\lambda+\rho, \alpha_{1}^{\vee}\right\rangle \in \mathbb{Z}_{<0}$ e a construção é semelhante.

Oberservemos se $\left\langle\lambda+\rho, \alpha_{1}^{\vee}\right\rangle=0$ então $L(\gamma, \mu)$ não é um módulo de $\Gamma$-relações. A afirmação a) é clara a partir da construção, enquanto b) e c) seguem da Proposição 1.3.14, Corolário 1.3.18 e Corolário 1.4.27.

\subsection{Família de módulos induzidos de $\Gamma_{s t}$-relações}

Nesta seção, fornecemos uma construção explícita de uma família de módulos simples limitados de $\Gamma$-relações induzidos parabolicamente.

Fixamos os conjuntos de números complexos $\left\{u_{i}\right\}_{i=1, \ldots, n+1}$ e $\left\{v_{i}\right\}_{i=1, \ldots, n}$ que satisfazem:

a) $u_{i}-v_{1} \notin \mathbb{Z}$ para qualquer $1 \leq i \leq n$.

b) $v_{j}-v_{j+1} \in \mathbb{Z}_{>0}$ para qualquer $1 \leq j<n$.

Seja $T(v)$ a tabela de Gelfand-Tsetlin com entradas

$$
v_{i j}= \begin{cases}u_{i}, & \text { se } j=1 \\ v_{j-1}, & \text { se } j \neq 1\end{cases}
$$

com $1 \leq j \leq i \leq n+1$.

Consideremos o conjunto de relações $\mathcal{Q}=\mathcal{Q}^{+} \cup \mathcal{Q}^{-}$, em que 


$$
\begin{array}{ll}
\mathcal{Q}^{+}:= & \{((i+1, j) ;(i, j)) \mid 2 \leq j \leq i \leq n\} \\
\mathcal{Q}^{-}:= & \{((i, j) ;(i+1, j+1)) \mid 2 \leq j \leq i \leq n\} .
\end{array}
$$

Exemplo 3.6.1. Para $n=4$, a tabela $T(v)$ é:

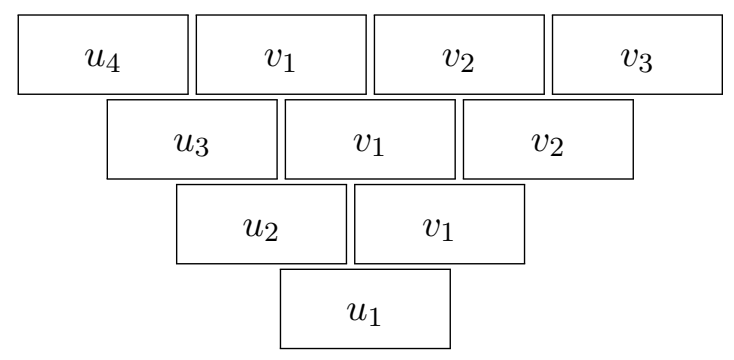

e o grafo $G(\mathcal{Q})$ é:

$(4,2)$

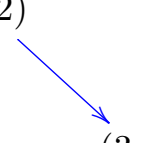

$(3,2)$

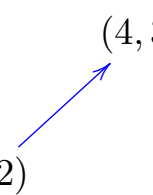

$(4,3)$

$(4,4)$

A seguinte afirmação segue imediatamente dos Lemas 3.1, 3.2 e 3.3 em [Maz03].

Lema 3.6.2. Seja $T(v)$ uma tabela de Gelfand-Tsetlin (3.4). Então $V_{\mathcal{Q}}(T(v))$ é um $\mathfrak{g}$-módulo limitado denso. Além disso, $V_{\mathcal{Q}}(T(v))$ é simples se, e somente se, $u_{i}-u_{i+1} \notin \mathbb{Z}$ para todo $1 \leq i \leq n$.

Corolário 3.6.3. Seja $\mathcal{C}$ um conjunto admissivel de relações que contém $\mathcal{Q}$ e $T(v)$ uma $\mathcal{C}$-realização, então $V_{\mathcal{C}}(T(v))$ é um submódulo de $V_{\mathcal{Q}}(T(v))$ e, portanto, um módulo limitado. Em particular, $V_{\mathcal{C}}(T(v))$ tem comprimento finito.

Demonstração. Por hipótese $\mathcal{Q} \subset \mathcal{C}$, então $V_{\mathcal{C}}(T(v))$ é um submódulo de $V_{\mathcal{Q}}(T(v))$ e sendo $V_{\mathcal{Q}}(T(v))$ um módulo limitado (pelo Lema 3.6.2), temos que $V_{\mathcal{C}}(T(v))$ um módulo limitado, portanto $V_{\mathcal{C}}(T(v)$ ) tem comprimento finito, já que módulos limitados tem (veja Seção 4 em [Fer90]).

Para $m \in\{2 \ldots, n\}$ consideramos a tabela $T^{m}(v)$ como em (3.4) satisfazendo as condições

$$
u_{i}=u_{m} \text { para } i=m+1, \ldots, n+1, \text { e } u_{i}-u_{i+1} \notin \mathbb{Z} \text { para } i=1,2, \ldots, m-1 .
$$

Seja $\mathcal{C}^{m}=\mathcal{Q} \cup\{((i+1,1) ;(i, 1)) \mid m \leq i \leq n\}$

Lema 3.6.4. O módulo de relações $V_{\mathcal{C}^{m}}\left(T^{m}(v)\right)$ é simples e limitado.

Demonstração. Dado que $T^{m}(v)$ é uma $\mathcal{C}^{m}$-realização e $\mathcal{C}^{m}$ é o conjunto admissível maximal satisfeito por $T^{m}(v)$, temos que $V_{\mathcal{C}^{m}}\left(T^{m}(v)\right)$ é um módulo de relações simples, pelo Teorema 3.3.19. Por outro lado, $\mathcal{C}^{m}$ é um conjunto admissível contendo $\mathcal{Q}$, então $V_{\mathcal{C}^{m}}\left(T^{m}(v)\right.$ ) é limitado (Corolário 3.6.3). 
Consideramos o subconjunto $\Sigma_{m}=\left\{\alpha_{1}, \ldots, \alpha_{m-1}\right\} \subset \pi$ de raízes simples e a subálgebra parabólica correspondente $\mathfrak{p}_{m}=\mathfrak{p}_{\Sigma_{m}} \subset \mathfrak{g}$. Então $\mathfrak{p}_{m}$ tem a subálgebra de Levi isomorfa com $\mathfrak{s l}_{m}+\mathfrak{h}$. Temos

Teorema 3.6.5 (Teorema 5.8 em [FHR20]). Para cada $m \in\{2, \ldots, n\}$, o módulo $V_{\mathcal{C}^{m}}\left(T^{m}(v)\right)$ é isomorfo com $L_{\mathfrak{p}_{m}}^{\mathfrak{g}}\left(\Sigma_{m}, V\right)$ para algum $\mathfrak{s l}_{m}$-módulo simples cuspidal $V$.

Demonstração. Por construção, $\mathcal{C}^{m}$ é o conjunto máximo de relações admissíveis satisfeitas por $T^{m}(v)$. Consequentemente, $V_{\mathcal{C}^{m}}\left(T^{m}(v)\right)$ é um $\mathfrak{g}$-módulo simples de Gelfand-Tsetlin pelo Teorema 3.3.19. Seja $\mathcal{D}^{m}=\mathcal{D}^{+} \cup \mathcal{D}^{-}$, em que

$$
\begin{aligned}
& \mathcal{D}^{+}=\{((i+1, j) ;(i, j)) \mid 2 \leq j \leq i \leq m-1\} \\
& \mathcal{D}^{-}=\{((i, j) ;(i+1, j+1)) \mid 2 \leq j \leq i \leq m-1\} .
\end{aligned}
$$

Então $V=V_{\mathcal{D}^{m}}\left(T^{m}(v)\right)=\operatorname{span}_{\mathbb{C}} \mathcal{B}_{\mathcal{D}^{m}}\left(T^{m}(v)\right)$ é um $\mathfrak{s l}_{m}$-módulo simples cuspidal pelo Lema 3.6.2, em que $\mathcal{B}_{\mathcal{D}^{m}}\left(T^{m}(v)\right)$ denota o conjunto de $\mathcal{D}^{m}$-realizações da forma $T^{m}(v+z), z \in \mathbb{Z}_{0}^{\frac{m(m+1)}{2}}$. Das fórmulas (3.3) segue que $V$ é um $\mathfrak{p}_{m}$-módulo com a ação trivial de $\mathfrak{u}_{\Sigma_{m}}^{+}$. Portanto, temos um homomorfismo

$$
\phi: M_{\mathfrak{p}}^{\mathfrak{g}}(\Sigma, V) \rightarrow V_{\mathcal{C}^{m}}\left(T^{m}(v)\right)
$$

de $U(\mathfrak{g})$-módulos, tal que $\phi\left(u \otimes T^{m}(v)\right)=u T^{m}(v)$ para todo $u \in U(\mathfrak{g})$. Como $V_{\mathcal{C}^{m}}\left(T^{m}(v)\right)$ é um $\mathfrak{g}$-módulo simples, então $\phi$ é sobrejetivo, e $L_{\mathfrak{p}}^{\mathfrak{g}}(\Sigma, V) \simeq V_{\mathcal{C}^{m}}\left(T^{m}(v)\right)$.

\subsection{Localização de módulos de peso máximo com relação a $\alpha_{1 m}$}

Nesta seção aplicaremos os funtores de localização e de torção $T_{\alpha}$ (veja Definição 1.3.1) aos módulos de peso máximo limitados de $\Gamma$-relações. Mas antes precisamos estabelecer condições nas bases dos módulos de relações para que faça sentido aplicar tais funtores ou para garantir a existência de inversos. Por tal motivo estudaremos as condições de injetividade e sobrejetividade dos operadores $E_{m 1}$, com $m \in\{2,3, \ldots, n+1\}$.

Notação 3.7.1. Para qualquer $2<m \leq n+1$ e qualquer $k \leq m-1$ fixamos $i_{k} \in\{1, \ldots, k\}$. Associado ao conjunto $\left\{i_{1}, \ldots, i_{m-1}\right\}$, definimos

$$
\varepsilon\left(i_{1}, \ldots, i_{m-1}\right):=-\delta^{1 i_{1}}-\delta^{2 i_{2}}-\delta^{3 i_{3}}-\ldots-\delta^{m-1, i_{m-1}} \in \mathbb{Z}_{0}^{\frac{(n+1)(n+2)}{2}} .
$$


Suponhamos que $T(v)$ seja uma $\mathcal{C}$-realização para um conjunto admissível de relações $\mathcal{C}$. Para cada $T(w) \in \mathcal{B}_{\mathcal{C}}(T(v))$ e qualquer $1 \leq i_{k} \leq k \leq m-1$, definimos

$$
a\left(w, i_{1}, \ldots, i_{m-1}\right):=\left\{\begin{array}{cl}
0, & \text { se } T\left(w+\varepsilon\left(i_{1}, \ldots, i_{m-1}\right)\right) \notin \mathcal{B}_{\mathcal{C}}(T(v)) \\
\prod_{s=2}^{m-1} \frac{\prod_{t \neq i_{s-1}}^{s-1}\left(w_{s i_{s}}-w_{s-1, t}\right)}{\prod_{t \neq i_{s}}^{s}\left(w_{s i_{s}}-w_{s t}\right)}, & \text { se } T\left(w+\varepsilon\left(i_{1}, \ldots, i_{m-1}\right)\right) \in \mathcal{B}_{\mathcal{C}}(T(v)) .
\end{array}\right.
$$

Pode-se verificar, por computação direta, o seguinte resultado análogo à Proposição 3.13 em [FGR16].

Proposição 3.7.2 (Proposição 5.9 em [FHR20]). Seja $\mathcal{C}$ um conjunto de relações admissivel e T $(v)$ uma $\mathcal{C}$-realização. $\operatorname{Se} T(w) \in \mathcal{B}_{\mathcal{C}}(T(v))$, então

$$
E_{m 1}(T(w))=\sum_{\substack{k=1, \ldots, m-1 \\\left(i_{1}, \ldots, i_{m-1} \in\{1, \ldots, k\}^{m-1}\right.}} a\left(w, i_{1}, \ldots, i_{m-1}\right) T\left(w+\varepsilon\left(i_{1}, \ldots, i_{m-1}\right)\right),
$$

para $m \in\{3, \ldots, n+1\}$.

Lembramos que $\mathcal{Q}=\{((i+1, j) ;(i, j)) \mid 2 \leq j \leq i \leq n\} \cup\{((i, j) ;(i+1, j+1)) \mid 2 \leq j \leq i \leq n\}$.

Teorema 3.7.3 (Teorema 5.10 em [FHR20]). Seja T(v) a tabela de Gelfand-Tsetlin da forma (3.4), $\mathcal{C}$ um conjunto admissível de relaçôes contendo $\mathcal{Q}$ para o qual $T(v)$ é uma realização $\mathcal{C}$. Então,

a) $E_{m 1}$ age injetivamente em $V_{\mathcal{C}}(T(v))$ se, e somente se, $((m-1,1) ;(m, 1)) \notin \mathcal{C}$;

b) $E_{m 1}$ age sobrejetivamente em $V_{\mathcal{C}}(T(v))$ se, e somente se, $((m, 1) ;(m-1,1)) \notin \mathcal{C}$.

Demonstração. a) Para cada $T(w) \in \mathcal{B}_{\mathcal{C}}(T(v))$, temos que

$$
T(w+\varepsilon(1, \ldots, 1))=T\left(w-\delta^{11}-\delta^{21}-\delta^{31}-\ldots-\delta^{m-1,1}\right) \in \mathcal{B}_{\mathcal{C}}(T(v)) .
$$

De fato, $\left(w_{i+1,1}-1\right)-\left(w_{i 1}-1\right)=w_{i+1,1}-w_{i 1}$ para todo $i=1, \ldots, m-2, w_{m 1}-\left(w_{m-1,1}-1\right)=$ $w_{m 1}-w_{m-1,1}+1 \in \mathbb{Z}_{>0}$ (ou $\notin \mathbb{Z}$ respectivamente) e $\left(w_{i 1}-1\right)-w_{i+1,2}=w_{i 1}-w_{i+1,2}-1 \notin \mathbb{Z}$ para todo $i=1, \ldots, m-1$. Por outro lado, para cada $s \in\{3, \ldots m-1\}$ dado que $w_{s 1}-w_{s-1,2} \notin \mathbb{Z}$ e $w_{s-1,2}-w_{s-1, t} \in \mathbb{Z}$ para todo $t \in\{2, \ldots s-1\}$, temos que $w_{s 1}-w_{s-1, t} \notin \mathbb{Z}$ para todo $t \in\{2, \ldots s-1\}$, assim

$$
a(w, 1, \ldots, 1)=\prod_{s=2}^{m-1} \frac{\prod_{t=2}^{s-1}\left(w_{s 1}-w_{s-1, t}\right)}{\prod_{t=2}^{s}\left(w_{s 1}-w_{s t}\right)} \neq 0
$$

Por conseguinte, (Veja a formula (3.5))

$$
\begin{aligned}
E_{m 1}(T(w)) & =a(w, 1, \ldots, 1) T(w+\varepsilon(1, \ldots, 1)) \\
& +\sum_{k=2}^{m-1} \sum_{\left(i_{1}, \ldots, i_{m-1}\right) \in\left(\{1, \ldots, k\}^{m-1} \backslash\{(1, \ldots, 1)\}\right)} a\left(w, i_{1}, \ldots, i_{m-1}\right) T\left(w+\varepsilon\left(i_{1}, \ldots, i_{m-1}\right)\right) \neq 0 .
\end{aligned}
$$


Uma vez que,

$T(w+\varepsilon(1, \ldots, 1)) \neq T\left(w+\varepsilon\left(i_{1}, \ldots, i_{m-1}\right)\right)$ for all $\left(i_{1}, \ldots, i_{m-1}\right) \in\left(\{1, \ldots, k\}^{m-1} \backslash\{(1, \ldots, 1)\}\right)$. Agora suponhamos que $0 \neq u=\sum_{i \in I} c_{i} T\left(w^{i}\right) \in V_{\mathcal{C}}(T(v))$ com $c_{i} \neq 0$ para cada $i \in I$, então

$$
E_{m 1}(u)=\sum_{\substack{i \in I, k_{k=1, \ldots, m-1} \\\left(i_{1}, \ldots, i_{m-1}\right) \in\{1, \ldots, k\}^{m-1}}} c_{i} a\left(w^{i}, i_{1}, \ldots, i_{m-1}\right) T\left(w^{i}-\varepsilon\left(i_{1}, \ldots, i_{m-1}\right)\right) \neq 0
$$

De fato, temos que para cada $i \in I: c_{i} a\left(w^{i}, 1, \ldots, 1\right) T\left(w^{i}+\varepsilon(1, \ldots, 1)\right) \neq 0$ e

$T\left(w^{i}+\varepsilon(1, \ldots, 1)\right) \neq T\left(w^{i}+\varepsilon\left(i_{1}, \ldots, i_{m-1}\right)\right)$ for all $\left(i_{1}, \ldots, i_{m-1}\right) \in\left(\{1, \ldots, k\}^{m-1} \backslash\{(1, \ldots, 1)\}\right)$.

Além disso, como $T\left(w^{i}\right) \neq T\left(w^{j}\right)$ então $T\left(w^{i}+\varepsilon(1, \ldots, 1)\right) \neq T\left(w^{j}+\varepsilon(1, \ldots, 1)\right)$ para todo $i \neq j \in I$. Mais ainda, existe $i \in I$ tal que $T\left(w^{i}+\varepsilon(1, \ldots, 1)\right) \neq T\left(w^{j}+\varepsilon\left(i_{1}, \ldots, i_{m-1}\right)\right)$ para todo $j \neq i \in I$ e para todo $\left(i_{1}, \ldots, i_{m-1}\right) \in\left(\{1, \ldots, k\}^{m-1} \backslash\{(1, \ldots, 1)\}\right)$. Finalmente, suponhamos para cada $i \in I$ existe $j \in I$ tal que $j \neq i$ e $T\left(w^{i}+\varepsilon(1, \ldots, 1)\right)=T\left(w^{j}+\varepsilon\left(i_{1}, \ldots, i_{m-1}\right)\right)$ para $\operatorname{algum}\left(i_{1}, \ldots, i_{m-1}\right) \neq(1, \ldots, 1)$. Então existe $s \in\{2, \ldots m-1\}$ tal que $w_{s 1}^{i}-1=w_{s 1}^{j}$, logo

$$
\sum_{j \in I} w_{s 1}^{j}=\sum_{i \in I}\left(w_{s 1}^{i}-1\right)=\sum_{i \in I} w_{s 1}^{i}-\# I=\sum_{j \in I} w_{s 1}^{j}-\# I
$$

portanto, $\# I=0$, porém \#I é o número de elementos de um conjunto finito não-vazio $I$, assim temos uma contradição.

Reciprocamente, suponhamos que $((m-1,1) ;(m, 1)) \in \mathcal{C}$. Dado que

$$
\{((m-1, j) ;(m, j+1)) \mid j=2, \ldots, m-1\} \subset \mathcal{C},
$$

por hipótese, então existe $T(w) \in \mathcal{B}_{\mathcal{C}}(T(v))$ tal que $w_{m-1,1}-w_{m 1}=w_{m-1, j}-w_{m, j+1}=1$ para cada $j=2, \ldots m-1, \operatorname{logo} T\left(w+\varepsilon\left(i_{1}, \ldots, i_{m-1}\right)\right) \notin \mathcal{B}_{\mathcal{C}}(T(v))$ para cada $1 \leq i_{k} \leq k \leq m-1$. Portanto, $E_{m 1}(T(w))=0$.

b) Primeiro, observemos que para cada $T(w) \in \mathcal{B}_{\mathcal{C}}(T(v))$, desde que $((m, 1) ;(m-1,1)) \notin \mathcal{C}$, temos que

$$
T\left(w^{\prime}\right):=T(w-\varepsilon(1, \ldots, 1))=T\left(w+\delta^{11}+\delta^{21}+\ldots+\delta^{m-1,1}\right) \in \mathcal{B}_{\mathcal{C}}(T(v)) .
$$

De fato, $\left(w_{i+1,1}+1\right)-\left(w_{i 1}+1\right)=w_{i+1,1}-w_{i 1}$ para todo $i=1, \ldots, m-2,\left(w_{m-1,1}+1\right)-w_{m 1}=$ $w_{m-1,1}-w_{m 1}+1 \in \mathbb{Z}_{>0}$ (ou $\notin \mathbb{Z}$ respectivamente) e $\left(w_{i 1}+1\right)-w_{i+1,2}=w_{i 1}-w_{i+1,2}+1 \notin \mathbb{Z}$ para todo $i=1, \ldots, m-1$. Assim, podemos usar a seguinte fórmula obtida de (3.5)

$$
\begin{aligned}
& E_{m 1}\left(T\left(w^{\prime}\right)\right)=b(w, 1, \ldots, 1) T(w) \\
& +\sum_{\substack{k=2, \ldots, m-1 \\
\left(i_{2}, \ldots, i_{m-1}\right) \in\left(\{1, \ldots, k\}^{m-1} \backslash\{(1, \ldots, 1)\}\right)}} b\left(w, i_{2}, \ldots, i_{m-1}\right) T\left(w+\varepsilon^{\prime}\left(i_{2}, \ldots, i_{m-1}\right)\right) .
\end{aligned}
$$

em que

$$
\varepsilon^{\prime}\left(i_{2}, \ldots, i_{m-1}\right):=\delta^{21}-\delta^{2 i_{2}}+\delta^{31}-\delta^{3 i_{3}}+\ldots+\delta^{m-1,1}-\delta^{m-1, i_{m-1}} \in \mathbb{Z}_{0}^{\frac{(n+1)(n+2)}{2}} .
$$


e $b\left(w, i_{2}, \ldots, i_{m-1}\right):=\left\{\begin{array}{cl}0, & \text { se } T\left(w+\varepsilon^{\prime}\left(i_{2}, \ldots, i_{m-1}\right)\right) \notin \mathcal{B}_{\mathcal{C}}(T(v)) \\ \prod_{s=2}^{m-1} \frac{\prod_{t \neq i_{s-1}}^{s-1}\left(w_{s i_{s}}-w_{s-1, t}+\delta_{1 i_{s}}-\delta_{1 t}\right)}{\prod_{t \neq i_{s}}^{s}\left(w_{s i_{s}}-w_{s t}+\delta_{1 i_{s}}-\delta_{1 t}\right)}, & \text { se } T\left(w+\varepsilon^{\prime}\left(i_{2}, \ldots, i_{m-1}\right)\right) \in \mathcal{B}_{\mathcal{C}}(T(v)) .\end{array}\right.$

Em particular para cada $s \in\{3, \ldots m-1\}$, temos que $w_{s 1}-w_{s-1, t} \notin \mathbb{Z}$ para cada $t \in\{2, \ldots s-1\}$, portanto

$$
b(w, 1, \ldots, 1)=\prod_{s=2}^{m-1} \frac{\prod_{t=2}^{s-1}\left(w_{s 1}-w_{s-1, t}\right)}{\prod_{t=2}^{s}\left(w_{s 1}-w_{s t}+1\right)} \neq 0 .
$$

Mas, $T(w) \in \mathcal{B}_{\mathcal{C}}(T(v))$, logo: $w_{i j}-w_{i+1, j+1} \in\left\{1,2, \ldots, d_{i j}\right\}$, em que $d_{i j}:=v_{j-1}-v_{n+j-i-1}-$ $n+i+1 \in \mathbb{Z}_{>0}$ são fixados. Portanto, temos os seguintes casos: ${ }^{5}$

Caso I: $w_{22}-w_{33} \in\left\{1,2, \ldots, d_{22}\right\}$ e $w_{i j}-w_{i+1, j+1}=1$ para todo $3 \leq i \leq n$ e $2 \leq j \leq i$. Neste caso, observemos que $T\left(w+\varepsilon^{\prime}\left(i_{2}, \ldots, i_{m-1}\right)\right) \notin \mathcal{B}_{\mathcal{C}}(T(v))$ para todo $\left(i_{2}, \ldots, i_{m-1}\right)$ tal que $i_{k}>1$ para algum $k=3, \ldots, m-1$, uma vez que $\left(w_{i j}-1\right)-w_{i+1, j+1}=0$ para todo $3 \leq i \leq n-1 \mathrm{e}$ $2 \leq j \leq i$. Assim:

$$
E_{m 1}\left(T\left(w^{\prime}\right)\right)=b(w, 1,1, \ldots, 1) T(w)+b(w, 2,1, \ldots, 1) T\left(w+\delta^{21}-\delta^{22}\right)
$$

Primeiro, observemos que se $((1,1) ;(2,1)) \in \mathcal{C}$ ou $((3,1) ;(2,1)) \in \mathcal{C}$, então $T\left(w+\delta^{21}-\delta^{22}\right) \notin$ $\mathcal{B}_{\mathcal{C}}(T(v))$, em que $w_{11}-w_{21}=1$ ou $w_{31}-w_{21}=0$, portanto $b(w, 2,1, \ldots, 1)=0$ e $E_{m 1}\left(T\left(w^{\prime}\right)\right)=$ $b(w, 1,1, \ldots, 1) T(w)$. Assim,

$$
T(w)=E_{m 1}\left(\frac{1}{b(w, 1,1, \ldots, 1)} T\left(w^{\prime}\right)\right) .
$$

Portanto, é possível supor que $((1,1) ;(2,1)) \notin \mathcal{C}$ e $((3,1) ;(2,1)) \notin \mathcal{C}$ e consideremos os próximos sub-casos:

a) $w_{22}-w_{33}=1$.

Neste caso $T\left(w+\delta^{21}-\delta^{22}\right) \notin \mathcal{B}_{\mathcal{C}}(T(v))$, desde que $\left(w_{22}-1\right)-w_{33}=0$. Isto implica que: $E_{m 1}\left(T\left(w^{\prime}\right)\right)=b(w, 1,1, \ldots, 1) T(w)$.

$$
E_{m 1}\left(T\left(w^{\prime}\right)\right)=b(w, 1,1, \ldots, 1) T(w) \Leftrightarrow T(w)=E_{m 1}\left(\frac{1}{b(w, 1,1, \ldots, 1)} T\left(w^{\prime}\right)\right) .
$$

Assim,

$$
T(w)=E_{m 1}\left(\frac{1}{b(w, 1,1, \ldots, 1)} T\left(w^{\prime}\right)\right)
$$

b) $w_{22}-w_{33}=2$.

\footnotetext{
${ }^{5}$ De fato, o total de casos é: $1 \leq \prod_{2 \leq j \leq i \leq n-1} d_{i j}<\infty$
} 
Neste caso $T\left(w+\delta^{21}-\delta^{22}\right) \in \mathcal{B}_{\mathcal{C}}(T(v))$, uma vez que $\left(w_{22}-1\right)-w_{33}=w_{22}-w_{33}-1=1$, $w_{32}-\left(w_{22}-1\right)=w_{32}-w_{22}+1 \in \mathbb{Z}_{>0}$. Logo, aplicando o caso anterior

$$
T\left(w+\delta^{21}-\delta^{22}\right)=E_{m 1}\left(\frac{1}{b\left(w+\delta^{21}-\delta^{22}, 1,1, \ldots, 1\right)} T\left(w^{\prime}+\delta^{21}-\delta^{22}\right)\right)
$$

Assim,

$$
E_{m 1}\left(T\left(w^{\prime}\right)\right)=b(w, 1,1, \ldots, 1) T(w)+b(w, 2,1, \ldots, 1) T\left(w+\delta^{21}-\delta^{22}\right)
$$

Consequentemente,

$$
\begin{aligned}
T(w) & =\frac{1}{b(w, 1,1, \ldots, 1)} E_{m 1}\left(T\left(w^{\prime}\right)\right)-\frac{b(w, 2,1, \ldots, 1)}{b(w, 1,1, \ldots, 1)} T\left(w+\delta^{21}-\delta^{22}\right) \\
& =E_{m 1}\left(T\left(w_{0}\right)\right)
\end{aligned}
$$

em que $T\left(w_{0}\right) \in V_{\mathcal{C}}(T(v))$, uma vez que

$$
\begin{aligned}
T\left(w_{0}\right)= & \frac{1}{b(w, 1,1, \ldots, 1)} T\left(w^{\prime}\right) \\
& -\frac{b(w, 2,1, \ldots, 1)}{b(w, 1,1, \ldots, 1) b\left(w+\delta^{21}-\delta^{22}, 1,1, \ldots, 1\right)} T\left(w^{\prime}+\delta^{21}-\delta^{22}\right)
\end{aligned}
$$

c) $w_{22}-w_{33}=i \in\left\{2,3,4, \ldots, d_{22}\right\}$.

Neste caso $T\left(w+\delta^{21}-\delta^{22}\right) \in \mathcal{B}_{\mathcal{C}}(T(v))$, uma vez que $\left(w_{22}-1\right)-w_{33}=w_{22}-w_{33}-1=i-1$, $w_{32}-\left(w_{22}-1\right)=w_{32}-w_{22}+1 \in \mathbb{Z}_{>0}$. Assim, aplicando o caso $w_{22}-w_{33}=i-1$, temos que $T\left(w+\delta^{21}-\delta^{22}\right)=E_{m 1}\left(T\left(w_{1}\right)\right)$, em que $T\left(w_{1}\right)$ é uma tabela em $V_{\mathcal{C}}(T(v))$. Assim, $E_{m 1}\left(T\left(w^{\prime}\right)\right)=b(w, 1,1, \ldots, 1) T(w)+b(w, 2,1, \ldots, 1) T\left(w+\delta^{21}-\delta^{22}\right)$. Consequentemente, $T(w)=E_{m 1}\left(T\left(w_{0}\right)\right)$ para algum $T\left(w_{0}\right) \in V_{\mathcal{C}}(T(v))$.

$$
\begin{aligned}
T(w) & =\frac{1}{b(w, 1,1, \ldots, 1)} E_{m 1}\left(T\left(w^{\prime}\right)\right)-\frac{b(w, 2,1, \ldots, 1)}{b(w, 1,1, \ldots, 1)} T\left(w+\delta^{21}-\delta^{22}\right) \\
& =E_{m 1}\left(T\left(w_{0}\right)\right)
\end{aligned}
$$

em que $T\left(w_{0}\right) \in V_{\mathcal{C}}(T(v))$, uma vez que

$$
T\left(w_{0}\right)=\frac{1}{b(w, 1,1, \ldots, 1)} T\left(w^{\prime}\right)-\frac{b(w, 2,1, \ldots, 1)}{b(w, 1,1, \ldots, 1)} T\left(w_{1}\right)
$$

Assim, temos que para cada $T(w) \in \mathcal{B}_{\mathcal{C}}(T(v))$ tal que $w_{22}-w_{33} \in\left\{1,2, \ldots, d_{22}\right\}$ e $w_{i j}-$ $w_{i+1, j+1}=1$ para todo $3 \leq i \leq n-1$ e $2 \leq j \leq i$, existe uma tabela $T\left(w_{0}\right) \in V_{\mathcal{C}}(T(v))$ tal que $T(w)=E_{m 1}\left(T\left(w_{0}\right)\right)$

Caso II: $w_{22}-w_{33} \in\left\{1,2, \ldots, d_{22}\right\}, w_{32}-w_{43}=2$ e $w_{i j}-w_{i+1, j+1}=w_{33}-w_{44}=1$ para todo $4 \leq i \leq n-1$ e $2 \leq j \leq i$.

Neste caso, observemos que $T\left(w+\varepsilon^{\prime}\left(i_{2}, \ldots, i_{m-1}\right)\right) \notin \mathcal{B}_{\mathcal{C}}(T(v))$ para todo $\left(i_{2}, \ldots, i_{m-1}\right)$ tal que $i_{3}=3$ ou $i_{k}>1$ para cada $k=4, \ldots, m-1$, uma vez que $\left(w_{i j}-1\right)-w_{i+1, j+1}=\left(w_{33}-1\right)-w_{44}=0$ 
para todo $4 \leq i \leq n-1$ e $2 \leq j \leq i$. Assim:

$$
\begin{aligned}
E_{m 1}\left(T\left(w^{\prime}\right)\right)= & b(w, 1,1, \ldots, 1) T(w)+b(w, 2,1, \ldots, 1) T\left(w+\delta^{21}-\delta^{22}\right) \\
& +b(w, 2,2, \ldots, 1) T\left(w+\delta^{21}-\delta^{22}+\delta^{31}-\delta^{32}\right) \\
& +b(w, 1,2, \ldots, 1) T\left(w+\delta^{31}-\delta^{32}\right)
\end{aligned}
$$

Observemos que se $((2,1) ;(3,1)) \in \mathcal{C}$ ou $((4,1) ;(3,1)) \in \mathcal{C}$, então $T\left(w+\delta^{21}-\delta^{22}+\delta^{31}-\right.$ $\left.\delta^{32}\right) \notin B_{\mathcal{C}}(T(v))$ e $T\left(w+\delta^{31}-\delta^{32}\right) \notin B_{\mathcal{C}}(T(v))$, em que $w_{21}-w_{31}=1$ ou $w_{41}-w_{31}=$ 0 , portanto $b(w, 2,2, \ldots, 1)=b(w, 1,2, \ldots, 1)=0$ e $E_{m 1}\left(T\left(w^{\prime}\right)\right)=b(w, 1,1, \ldots, 1) T(w)+$ $b(w, 2,1, \ldots, 1) T\left(w+\delta^{21}-\delta^{22}\right)$. Mais ainda, se $((1,1) ;(2,1)) \in \mathcal{C}$ ou $((3,1) ;(2,1)) \in \mathcal{C}$ então,

$$
T(w)=E_{m 1}\left(\frac{1}{b(w, 1,1, \ldots, 1)} T\left(w^{\prime}\right)\right) .
$$

Logo, sem perda de generalidade, podemos supor que

$$
\{((1,1) ;(2,1)),((2,1) ;(3,1)),((3,1) ;(2,1)),((4,1) ;(3,1))\} \cap \mathcal{C}=\emptyset .
$$

Portanto, temos os sub-casos seguintes:

a) $w_{22}-w_{33}=1$.

Observemos que, $T\left(w+\delta^{21}-\delta^{22}\right)$ e $T\left(w+\delta^{21}-\delta^{22}+\delta^{31}-\delta^{32}\right)$ não são tabelas em $\mathcal{B}_{\mathcal{C}}(T(v))$, uma vez que $\left(w_{22}-1\right)-w_{33}=w_{22}-w_{33}-1=0$. Por outro lado, $T\left(w+\delta^{31}-\delta^{32}\right) \in \mathcal{B}_{\mathcal{C}}(T(v))$, uma vez que $w_{42}-\left(w_{32}-1\right)=w_{42}-w_{32}+1 \in \mathbb{Z}_{>0},\left(w_{32}-1\right)-w_{43}=w_{32}-w_{43}-1=1$, $\left(w_{32}-1\right)-w_{22}=\left(w_{43}+1\right)-\left(w_{33}+1\right)=w_{43}-w_{33} \in \mathbb{Z}_{\geq 0}$. Assim, pela aplicação do caso I-a:

$$
T\left(w+\delta^{31}-\delta^{32}\right)=E_{m 1}\left(\frac{1}{b\left(w+\delta^{31}-\delta^{32}, 1,1, \ldots, 1\right)} T\left(w^{\prime}+\delta^{31}-\delta^{32}\right)\right) .
$$

Assim, a fórmula pode ser reduzida a

$$
E_{m 1}\left(T\left(w^{\prime}\right)\right)=b(w, 1,1, \ldots, 1) T(w)+b(w, 1,2, \ldots, 1) T\left(w+\delta^{31}-\delta^{32}\right)
$$

Logo, resolvendo para $T(w)$, temos que :

$$
\begin{aligned}
T(w) & =\frac{1}{b(w, 1,1, \ldots, 1)} E_{m 1}\left(T\left(w^{\prime}\right)\right)-\frac{b(w, 1,2, \ldots, 1)}{b(w, 1,1, \ldots, 1)} T\left(w+\delta^{31}-\delta^{32}\right) \\
& =E_{m 1}\left(T\left(w_{0}\right)\right)
\end{aligned}
$$

em que $T\left(w_{0}\right) \in V_{\mathcal{C}}(T(v))$, desde que

$$
\begin{aligned}
T\left(w_{0}\right)= & \frac{1}{b(w, 1,1, \ldots, 1)} T\left(w^{\prime}\right) \\
& -\frac{b(w, 1,2, \ldots, 1)}{b(w, 1,1, \ldots, 1) b\left(w+\delta^{31}-\delta^{32}, 1,1, \ldots, 1\right) a_{0}(w)} T\left(w^{\prime}+\delta^{31}-\delta^{32}\right)
\end{aligned}
$$

b) $w_{22}-w_{33}=i \in\left\{2,3,4, \ldots, d_{22}\right\}$.

Observemos que, $T\left(w+\delta^{21}-\delta^{22}\right)$ e $T\left(w+\delta^{21}-\delta^{22}+\delta^{31}-\delta^{32}\right)$ são tabelas em $\mathcal{B}_{\mathcal{C}}(T(v))$, uma vez 
que $\left(w_{22}-1\right)-w_{33}=w_{22}-w_{33}-1=i-1 \in \mathbb{Z}_{>0},\left(w_{32}-1\right)-\left(w_{22}-1\right)=w_{32}-w_{22} \in \mathbb{Z}_{\geq 0}$, $w_{32}-\left(w_{22}-1\right)=w_{32}-w_{22}+1 \in \mathbb{Z}_{>0}, w_{42}-\left(w_{32}-1\right)=w_{42}-w_{32}+1 \in \mathbb{Z}_{>0} \mathrm{e}$ $\left(w_{32}-1\right)-w_{43}=w_{32}-w_{43}-1=1$. Por outro lado, $T\left(w+\delta^{31}-\delta^{32}\right) \in \mathcal{B}_{\mathcal{C}}(T(v))$ se, e somente se, $w_{43}-w_{44} \in \mathbb{Z}_{\geq i}$, uma vez que $w_{42}-\left(w_{32}-1\right)=w_{42}-w_{32}+1 \in \mathbb{Z}_{>0}$, $\left(w_{32}-1\right)-w_{43}=w_{32}-w_{43}-1=1$ e $\left(w_{32}-1\right)-w_{22}=w_{43}-w_{44}-i$. Assim, aplicando o caso I, existe $T\left(w_{j}\right) \in V_{\mathcal{C}}(T(v))$ para cada $j=1,2,3$ tal que: $T\left(w+\delta^{21}-\delta^{22}\right)=E_{m 1}\left(T\left(w_{1}\right)\right)$, $T\left(w+\delta^{21}-\delta^{22}+\delta^{31}-\delta^{32}\right)=E_{m 1}\left(T\left(w_{2}\right)\right)$ e $T\left(w+\delta^{31}-\delta^{32}\right)=E_{m 1}\left(T\left(w_{3}\right)\right){ }^{6}$

Assim, a fórmula pode ser escrita na forma a

$$
\begin{aligned}
E_{m 1}\left(T\left(w^{\prime}\right)\right)= & b(w, 1,1, \ldots, 1) T(w)+(w, 2,1, \ldots, 1) T\left(w+\delta^{21}-\delta^{22}\right) \\
& +b(w, 2,2, \ldots, 1) T\left(w+\delta^{21}-\delta^{22}\right. \\
& \left.+\delta^{31}-\delta^{32}\right)+b(w, 1,2, \ldots, 1) T\left(w+\delta^{31}-\delta^{32}\right) \\
= & b(w, 1,1, \ldots, 1) T(w)+b(w, 2,1, \ldots, 1) E_{m 1}\left(T\left(w_{1}\right)\right) \\
& +b(w, 2,2, \ldots, 1) E_{m 1}\left(T\left(w_{2}\right)\right) \\
& +b(w, 1,2, \ldots, 1) E_{m 1}\left(T\left(w_{3}\right)\right)
\end{aligned}
$$

Assim, resolvendo para $T(w)$ temos que :

$$
\begin{aligned}
T(w)= & E_{m 1}\left(\frac{1}{b(w, 1,1, \ldots, 1)} T\left(w^{\prime}\right)-\frac{b(w, 2,1, \ldots, 1)}{b(w, 1,1, \ldots, 1)} T\left(w_{1}\right)\right. \\
& \left.-\frac{b(w, 2,2, \ldots, 1)}{b(w, 1,1, \ldots, 1)} T\left(w_{2}\right)-\frac{b(w, 1,2, \ldots, 1)}{b(w, 1,1, \ldots, 1)} T\left(w_{3}\right)\right) \\
= & E_{m 1}\left(T\left(w_{0}\right)\right)
\end{aligned}
$$

em que $T\left(w_{0}\right) \in V_{\mathcal{C}}(T(v))$, desde que

$$
\begin{aligned}
T\left(w_{0}\right)= & \frac{1}{b(w, 1,1, \ldots, 1)} T\left(w^{\prime}\right)-\frac{b(w, 2,1, \ldots, 1)}{b(w, 1,1, \ldots, 1)} T\left(w_{1}\right)-\frac{b(w, 2,2, \ldots, 1)}{b(w, 1,1, \ldots, 1)} T\left(w_{2}\right) \\
& -\frac{b(w, 1,2, \ldots, 1)}{b(w, 1,1, \ldots, 1)} T\left(w_{3}\right)
\end{aligned}
$$

Assim, temos que para cada $T(w) \in \mathcal{B}_{\mathcal{C}}(T(v))$ tal que $w_{22}-w_{33} \in\left\{1,2, \ldots, d_{22}\right\}, w_{32}-w_{43}=2$ e $w_{i j}-w_{i+1, j+1}=w_{33}-w_{44}=1$ para todo $4 \leq i \leq n-1$ e $2 \leq j \leq i$, existe uma tabela $T\left(w_{0}\right) \in V_{\mathcal{C}}(T(v))$ tal que $T(w)=E_{m 1}\left(T\left(w_{0}\right)\right)$.

Caso II: $w_{22}-w_{33} \in\left\{1,2, \ldots, d_{22}\right\}, w_{32}-w_{43}=j \in\left\{2,3,4, \ldots, d_{32}\right\}$ e $w_{i j}-w_{i+1, j+1}=$ $w_{33}-w_{44}=1$ para todo $4 \leq i \leq n-1$ e $2 \leq j \leq i$.

Neste caso, observemos que $T\left(w+\varepsilon^{\prime}\left(i_{2}, \ldots, i_{m-1}\right)\right) \notin \mathcal{B}_{\mathcal{C}}(T(v))$ para todo $\left(i_{2}, \ldots, i_{m-1}\right)$ tal que $i_{3}=3$ ou $i_{k}>1$ para cada $k=4, \ldots, m-1$, uma vez que $\left(w_{i j}-1\right)-w_{i+1, j+1}=\left(w_{33}-1\right)-w_{44}=0$ para todo $4 \leq i \leq n-1$ e $2 \leq j \leq i$.

a) $w_{22}-w_{33}=1$.

Observemos que, $T\left(w+\delta^{21}-\delta^{22}\right)$ e $T\left(w+\delta^{21}-\delta^{22}+\delta^{31}-\delta^{32}\right)$ não são tabelas em $\mathcal{B}_{\mathcal{C}}(T(v))$, uma vez que $\left(w_{22}-1\right)-w_{33}=w_{22}-w_{33}-1=0$. Por outro lado, $T\left(w+\delta^{31}-\delta^{32}\right) \in \mathcal{B}_{\mathcal{C}}(T(v))$,

\footnotetext{
${ }^{6}$ Se $w_{43}-w_{44}<i$, então $T\left(w_{3}\right)=0$.
} 
uma vez que $w_{42}-\left(w_{32}-1\right)=w_{42}-w_{32}+1 \in \mathbb{Z}_{>0},\left(w_{32}-1\right)-w_{43}=w_{32}-w_{43}-1=j-1 \geq 2$, $\left(w_{32}-1\right)-w_{22}=w_{43}-w_{44}+j-3 \in \mathbb{Z}_{>0}$. Assim, aplicando o caso I-a, temos que existe $T\left(w_{1}\right) \in V_{\mathcal{C}}(T(v))$, tal que $: T\left(w+\delta^{31}-\delta^{32}\right)=E_{m 1}\left(T\left(w_{1}\right)\right)$.

Assim, $\left.E_{m 1}\left(T\left(w^{\prime}\right)\right)\right)=b(w, 1,1, \ldots, 1) T(w)+b(w, 1,2, \ldots, 1) E_{m 1}\left(T\left(w_{1}\right)\right)$. Assim, a fórmula pode ser reduzida a forma:

$$
\begin{aligned}
\left.E_{m 1}\left(T\left(w^{\prime}\right)\right)\right) & =b(w, 1,1, \ldots, 1) T(w)+b(w, 1,2, \ldots, 1) T\left(w+\delta^{31}-\delta^{32}\right) \\
& =b(w, 1,1, \ldots, 1) T(w)+b(w, 1,2, \ldots, 1) E_{m 1}\left(T\left(w_{1}\right)\right)
\end{aligned}
$$

Assim, resolvendo para $T(w)$, temos que:

$$
\begin{aligned}
T(w) & =E_{m 1}\left(\frac{1}{b(w, 1,1, \ldots, 1)} T\left(w^{\prime}\right)-\frac{b(w, 1,2, \ldots, 1)}{b(w, 1,1, \ldots, 1)} T\left(w_{1}\right)\right) \\
& =E_{m 1}\left(T\left(w_{0}\right)\right)
\end{aligned}
$$

em que $T\left(w_{0}\right)=\frac{1}{b(w, 1,1, \ldots, 1)} T\left(w^{\prime}\right)-\frac{b(w, 1,2, \ldots, 1)}{b(w, 1,1, \ldots, 1)} T\left(w_{1}\right) \in V_{\mathcal{C}}(T(v))$.

b) $w_{22}-w_{33}=i \in\left\{2,3, \ldots, d_{22}\right\}$.

Observemos que, $T\left(w+\delta^{21}-\delta^{22}\right)$ e $T\left(w+\delta^{21}-\delta^{22}+\delta^{31}-\delta^{32}\right)$ são tabelas em $\mathcal{B}_{\mathcal{C}}(T(v))$, uma vez que $\left(w_{22}-1\right)-w_{33}=w_{22}-w_{33}-1=i-1 \in \mathbb{Z}_{>0},\left(w_{32}-1\right)-\left(w_{22}-1\right)=w_{32}-w_{22} \in \mathbb{Z}_{\geq 0}$, $w_{32}-\left(w_{22}-1\right)=w_{32}-w_{22}+1 \in \mathbb{Z}_{>0}, w_{42}-\left(w_{32}-1\right)=w_{42}-w_{32}+1 \in \mathbb{Z}_{>0} \mathrm{e}$ $\left(w_{32}-1\right)-w_{43}=w_{32}-w_{43}-1=j-1 \in \mathbb{Z}_{>1}$. Por outro lado, $T\left(w+\delta^{31}-\delta^{32}\right) \in \mathcal{B}_{\mathcal{C}}(T(v))$ se, e somente se, $w_{43}-w_{44} \geq i-j+2$, uma vez que $w_{42}-\left(w_{32}-1\right)=w_{42}-w_{32}+1 \in \mathbb{Z}_{>0}$, $\left(w_{32}-1\right)-w_{43}=w_{32}-w_{43}-1=1$ e $\left(w_{32}-1\right)-w_{22}=w_{43}-w_{44}+j-i-2$. Assim, aplicando o caso s I e II-a), existe $T\left(w_{k}\right) \in V_{\mathcal{C}}(T(v))$ para cada $k=1,2,3$ tal que : $T\left(w+\delta^{21}-\delta^{22}\right)=$ $E_{m 1}\left(T\left(w_{1}\right)\right), T\left(w+\delta^{21}-\delta^{22}+\delta^{31}-\delta^{32}\right)=E_{m 1}\left(T\left(w_{2}\right)\right)$ e $T\left(w+\delta^{31}-\delta^{32}\right)=E_{m 1}\left(T\left(w_{3}\right)\right){ }^{7}$

Assim, a fórmula pode ser escrita como

$$
\begin{aligned}
E_{m 1}\left(T\left(w^{\prime}\right)\right)= & b(w, 1,1, \ldots, 1) T(w)+b(w, 2,1, \ldots, 1) T\left(w+\delta^{21}-\delta^{22}\right) \\
& +b(w, 2,2, \ldots, 1) T\left(w+\delta^{21}-\delta^{22}\right. \\
& \left.+\delta^{31}-\delta^{32}\right)+b(w, 1,2, \ldots, 1) T\left(w+\delta^{31}-\delta^{32}\right) \\
= & b(w, 1,1, \ldots, 1) T(w)+b(w, 2,1, \ldots, 1) E_{m 1}\left(T\left(w_{1}\right)\right) \\
& +b(w, 2,2, \ldots, 1) E_{m 1}\left(T\left(w_{2}\right)\right)+b(w, 1,2, \ldots, 1) E_{m 1}\left(T\left(w_{3}\right)\right)
\end{aligned}
$$

Assim, temos que para cada $T(w) \in \mathcal{B}_{\mathcal{C}}(T(v))$ tal que $w_{22}-w_{33} \in\left\{1,2, \ldots, d_{22}\right\}, w_{32}-w_{43}=$ $j \in\left\{3,4, \ldots, d_{32}\right\}$ e $w_{i j}-w_{i+1, j+1}=w_{33}-w_{44}=1$ para todo $4 \leq i \leq n-1$ e $2 \leq j \leq i$, existe uma tabela $T\left(w_{0}\right) \in V_{\mathcal{C}}(T(v))$ tal que $T(w)=E_{m 1}\left(T\left(w_{0}\right)\right)$.

Caso III: $w_{33}-w_{44}=k \in\left\{2,3, \ldots, d_{33}\right\}, w_{22}-w_{33} \in\left\{1,2, \ldots, d_{22}-k+1\right\}^{8}, w_{32}-w_{43} \in$ $\left\{1,2, \ldots, d_{32}\right\}$, e $w_{i j}-w_{i+1, j+1}=1$ para todo $4 \leq i \leq n-1$ e $2 \leq j \leq i$.

Neste caso, observemos que $T\left(w+\varepsilon^{\prime}\left(i_{2}, \ldots, i_{m-1}\right)\right) \notin \mathcal{B}_{\mathcal{C}}(T(v))$ para todo $\left(i_{2}, \ldots, i_{m-1}\right)$ tal que $i_{k}>1$ para cada $k=4, \ldots, m-1$, uma vez que $\left(w_{i j}-1\right)-w_{i+1, j+1}=0$ para todo $4 \leq i \leq n-1$ e $2 \leq j \leq i$.

\footnotetext{
${ }^{7}$ Se $w_{43}-w_{44}<i-j+2$, então $T\left(w_{3}\right)=0$.

${ }^{8} \mathrm{Se} d_{22} \geq k$, não precisamos considerar este caso e a prova está completa.
} 
Por outro lado, temos que $T\left(w+\delta^{31}-\delta^{33}\right)$ e $T\left(w+\delta^{21}-\delta^{22}+\delta^{31}-\delta^{33}\right)$ são tabelas em $\mathcal{B}_{\mathcal{C}}(T(v))$, uma vez que $w_{22}-\left(w_{33}-1\right)=w_{22}-w_{33}+1 \in\left\{2,3, \ldots, d_{22}\right\}, w_{43}-\left(w_{33}-1\right)=w_{43}-w_{33}+1 \in \mathbb{Z}_{>0}$, $\left(w_{33}-1\right)-w_{44}=w_{33}-w_{44}-1=1,\left(w_{22}-1\right)-\left(w_{33}-1\right)=w_{22}-w_{33}$ e $w_{32}-\left(w_{22}-1\right)=$ $w_{32}-w_{22}+1 \in \mathbb{Z}_{>0}$. Mais ainda, para esta tabela temos que $\left(w_{33}-1\right)-w_{44}=w_{33}-w_{44}-1=k-1$, $w_{32}-w_{43} \in\left\{1,2, \ldots, d_{32}\right\}$, Assim, aplicando o caso II, existe uma tabela $T\left(w_{1}\right)$ e $T\left(w_{2}\right)$ em $V_{\mathcal{C}}(T(v))$ tal que $T\left(w+\delta^{21}-\delta^{22}+\delta^{31}-\delta^{33}\right)=E_{m 1}\left(T\left(w_{1}\right)\right)$ e $T\left(w+\delta^{31}-\delta^{33}\right)=E_{m 1}\left(T\left(w_{2}\right)\right)$.

Assim, a fórmula pode ser escrita na forma:

$$
\begin{aligned}
E_{m 1}\left(T\left(w^{\prime}\right)\right)= & b(w, 1,1, \ldots, 1) T(w)+(w, 2,1, \ldots, 1) T\left(w+\delta^{21}-\delta^{22}\right) \\
& +b(w, 2,2, \ldots, 1) T\left(w+\delta^{21}-\delta^{22}+\delta^{31}-\delta^{32}\right) \\
& +b(w, 1,2, \ldots, 1) T\left(w+\delta^{31}-\delta^{32}\right) \\
& +b(w, 2,3, \ldots, 1) T\left(w+\delta^{21}-\delta^{22}+\delta^{31}-\delta^{33}\right) \\
& +b(w, 1,3, \ldots, 1) T\left(w+\delta^{31}-\delta^{33}\right)
\end{aligned}
$$

e reduzida para

$$
\begin{aligned}
E_{m 1}\left(T\left(w^{\prime \prime}\right)\right)= & b(w, 1,1, \ldots, 1) T(w)+(w, 2,1, \ldots, 1) T\left(w+\delta^{21}-\delta^{22}\right) \\
& +b(w, 2,2, \ldots, 1) T\left(w+\delta^{21}-\delta^{22}+\delta^{31}-\delta^{32}\right) \\
& +b(w, 1,2, \ldots, 1) T\left(w+\delta^{31}-\delta^{32}\right)
\end{aligned}
$$

em que $T\left(w^{\prime \prime}\right)=T\left(w^{\prime}\right)-b(w, 2,3, \ldots, 1) T\left(w_{1}\right)-b(w, 1,3, \ldots, 1) T\left(w_{2}\right) \in V_{\mathcal{C}}(T(v))$.

Podemos usar o mesmo argumento do caso acima para concluir a existência de $T\left(w_{0}\right) \in V_{\mathcal{C}}(T(v))$, para cada $T(w) \in \mathcal{B}_{\mathcal{C}}(T(v))$ satisfazendo $w_{22}-w_{33} \in\left\{1,2, \ldots, d_{22}\right\}, w_{32}-w_{43} \in\left\{1,2, \ldots, d_{32}\right\}$, $w_{33}-w_{44} \in\left\{1,2, \ldots, d_{33}\right\}$ e $w_{i j}-w_{i+1, j+1}=1$ para todo $4 \leq i \leq n-1$ e $2 \leq j \leq i$, tal que $T(w)=E_{m 1}\left(T\left(w_{0}\right)\right)$.

Repetindo o processo, após um número finito de vezes temos que para todo $T(w) \in \mathcal{B}_{\mathcal{C}}(T(v))$, existe $T\left(w_{0}\right) \in V_{\mathcal{C}}(T(v))$ tal que $T(w)=E_{m 1}\left(T\left(w_{0}\right)\right)$. Assim, para cada $u \in V_{\mathcal{C}}(T(v))$, temos que

$$
u=\sum_{i \in I} T\left(w_{i}\right)=\sum_{i \in I} a_{i} E_{m 1}\left(T\left(w_{0}^{i}\right)\right)=E_{m 1}\left(\sum_{i \in I} a_{i} T\left(w_{0}^{i}\right)\right) .
$$

Finalmente, provamos que se $E_{m 1}$ age sobrejetivamente em $V_{\mathcal{C}}(T(v))$ então $((m, 1) ;(m-1,1)) \notin \mathcal{C}$. Por contradição, suponhamos que $((m, 1) ;(m-1,1)) \in \mathcal{C}$ e escolhamos $T(w) \in \mathcal{B}_{\mathcal{C}}(T(v))$ tal que $w_{m-1,1}=w_{m 1}$. Por hipótese, sabemos que $((m, j) ;(m-1, j)) \in \mathcal{C}$ para todo $j=2, \ldots, m-1$, sem perda de generalidade, podemos assumir que $w_{m-1, j}=w_{m, j}$ para todo $j=2, \ldots, m-1$. Por outro lado, existe $u \in V_{\mathcal{C}}(T(v))$, tal que

$$
E_{m 1}(u)=\sum_{\substack{i \in I, k=1, \ldots, m-1 \\\left(i_{1}, \ldots, i_{m-1}\right) \in\{1, \ldots, k\}^{m-1}}} c_{i} a\left(w^{i}, i_{1}, \ldots, i_{m-1}\right) T\left(w^{i}-\varepsilon\left(i_{1}, \ldots, i_{m-1}\right)\right)=T(w) .
$$

Portanto, $T(w)=T\left(w^{i}-\varepsilon\left(i_{1}, \ldots, i_{m-1}\right)\right)$ para algum $i \in I$ e $1 \leq i_{k} \leq k \leq m-1$. Então existe $j=1, \ldots, m-1$, tal que $0=w_{m j}-w_{m-1, j}=w_{m j}^{i}-w_{m-1, j}^{i}+1>0$. 
Observação 3.7.4. No Teorema 3.7.3 o conjunto de relações $\mathcal{C}$ satisfeito por $T(v)$ não precisa ser maximal. É o caso, por exemplo, quando $u_{i+1}-u_{i} \in \mathbb{Z}_{\geq 0}$ para qualquer $1 \leq i<n$ e $\mathcal{C}=$ $\{((i+1,1) ;(i, 1)) \mid 1 \leq i \leq n\}$ ou $u_{i+1}-u_{i} \notin \mathbb{Z}$ para qualquer $1 \leq i<n$ e $\mathcal{C}=\emptyset$.

Corolário 3.7.5. Nas mesmas condições do Teorema 3.7.3. $E_{m 1}$ age bijetivamente em $V_{\mathcal{C}}(T(v))$ se, e somente se,

$$
\{((m-1,1) ;(m, 1)),((m, 1) ;(m-1,1))\} \cap \mathcal{C}=\emptyset .
$$

Demonstração. Segue do Teorema 3.7.3.

Seja $F:=\left\{E_{m_{i} 1} \mid i=1, \ldots, k\right\}$ de modo que $m_{i} \in\{2, \ldots, n+1\}$ para cada $i=1, \ldots, k$, temos

Proposição 3.7.6 (Proposição 5.12 em [FHR20]). Seja $\lambda \in \mathfrak{h}^{*}$.

a) Se $\lambda$ satisfaz as condições b), c) ou e) para $i=1$ do Corolário 3.4.10, então $D_{F} L(\lambda)$ é um módulo de $\Gamma$-relações limitado.

b) Seja $M=L(\lambda)$. Se $E_{m 1}$ age injetivamente em $M$ para algum $m \in\{2, \ldots, n+1\}$, então $T_{\alpha}(M)$ é um módulo de $\Gamma$-relações simples na categoria $\mathcal{O}$, em que $\alpha=\alpha_{1, m-1}$.

Demonstração. Suponhamos que $\lambda$ satisfaça a condição b) do Corolário 3.4.10 (a prova dos outros casos é semelhante). Então $L(\lambda) \simeq V_{\mathcal{C}}(T(v))$ em que $T(v)$ é a tabela de Gelfand-Tsetlin (3.4) tal que $u_{i}=u_{1}$ para todo $i=2,3, \ldots, n+1$ e $\mathcal{C}=\mathcal{Q} \cup\{((i+1,1) ;(i, 1)) \mid 1 \leq i \leq n\}$. Seja $M=V_{\mathcal{C}}(T(v))$ e $N=V_{\mathcal{D}}(T(v))$, em que $\mathcal{D}=\mathcal{C} \backslash\left\{\left(\left(m_{i}, 1\right) ;\left(m_{i}-1,1\right)\right) \mid i=1, \ldots, k\right\}$. Então $M \subset D_{F} M \subset N$ pelo Teorema 3.7.3.

Suponhamos primeiro que $k=1$. Então $F=F_{m}=\left\{E_{m 1}\right\}$. Como $E_{m 1}$ é injetivo em $M$, mas não bijetivo, concluímos pelo Teorema 3.7.3 que $M$ é um submódulo próprio de $D_{F_{m}} M$. Por outro lado, consideremos o conjunto de relações $\mathcal{D}_{m}=\mathcal{D} \cup\{((m-1,1) ;(m, 1))\}$. Então $V_{\mathcal{D}_{m}}\left(T\left(v+\delta^{m-1,1}\right)\right)$ é um módulo simples pelo Teorema 3.3.19, e $V_{\mathcal{D}_{m}}\left(T\left(v+\delta^{m-1,1}\right)\right) \simeq N / M$. Portanto, $M$ é um submódulo maximal de $N$. Finalmente, dado que $E_{m 1}$ age bijetivamente em $N$, temos que $N \simeq D_{F_{m}} M$ pela Proposição 1.4.22. Isso completa a prova no caso $k=1$.

Agora, suponhamos que a afirmação seja verdadeira para todo subconjunto de $F \operatorname{com} k-1$ elementos. Seja $F_{i}:=F \backslash\left\{E_{m_{i} 1}\right\}$ e $\mathcal{D}_{i}:=\mathcal{D} \cup\left\{\left(\left(m_{i}, 1\right) ;\left(m_{i}-1,1\right)\right)\right\}$ para qualquer $i=1, \ldots, k$. Então $D_{F_{i}} M \simeq V_{\mathcal{D}_{i}}(T(v))$. Seja $L=V_{\mathcal{D}_{1}}(T(v))+\cdots+V_{\mathcal{D}_{k}}(T(v))$. Como $D_{F_{i}} M \subset D_{\left\{E_{m_{i}}\right\}} D_{F_{i}} M \simeq D_{F} M$, temos que $L \subset D_{F} M$. Uma vez que $L$ não é $F$-bijetivo, então $L$ é um submódulo próprio de $D_{F} M$. Por outro lado, seja $T(w)=T\left(v+i_{1} \delta^{m_{1}-1,1}+\left(i_{1}-1\right) \delta^{m_{2}-1,1}+\cdots+\delta^{m_{i_{1}}-1,1}+\cdots+\left(k-i_{s}+\right.\right.$ 1) $\left.\delta^{m_{i_{s}+1}-1,1}+\cdots+\delta^{m_{k}-1,1}\right)$. Consideremos $\mathcal{A}=\mathcal{D} \cup\left\{\left(\left(m_{i}-1,1\right) ;\left(m_{i}, 1\right)\right) \mid i=1, \ldots, k\right\}$, em que $\left\{m_{i} \mid i=1,2, \ldots k\right\}=\left\{m_{1}, \ldots, m_{i_{1}}\right\} \cup \cdots \cup\left\{m_{i_{s}}, \ldots, m_{k}\right\}$ é uma união disjunta de conjuntos com elementos consecutivos. Então $\mathcal{A}$ é um conjunto de relações maximal satisfeitas por $T(w)$ e $V_{\mathcal{A}}(T(w))$ é um módulo simples pelo Teorema 3.3.19. Como $N / L \simeq V_{\mathcal{A}}(T(w))$, concluímos que $L$ é um submódulo maximal de $N$ e $D_{F} M \simeq N$ pela Proposição 1.4.22.

Observação 3.7.7. Compare o item b) da Proposição anterior com o Teorema 1.3.3. 


\subsection{Realização de famílias de módulos de $\Gamma_{s t}$-relações para $\mathfrak{s l}_{4}$}

A seguir forneceremos uma realização explícita de alguns $\mathfrak{s l}_{4}$-módulos simples de $\Gamma$-relações com espaços de peso de dimensão finita (tanto limitados, como não limitados), assim como módulos com espaços de peso dimensão infinita, cada um destes correspondendo a um conjunto admissível.

Proposição 3.8.1. Seja $L(\lambda)=L\left(\lambda_{1}, \lambda_{2}, \lambda_{3}\right)$, em que $\lambda_{1}=\left\langle\lambda, \alpha_{1}^{\vee}\right\rangle \notin \mathbb{Z}, \lambda_{2}=\left\langle\lambda, \alpha_{2}^{\vee}\right\rangle \in \mathbb{Z}_{\geq 0}$ e $\lambda_{3}=\left\langle\lambda, \alpha_{3}^{\vee}\right\rangle \in \mathbb{Z}_{\geq 0}$. L $(\lambda)$ é um módulo de $\Gamma$-relações limitado de grau $\frac{1}{2}\left(\lambda_{2}+1\right)\left(\lambda_{3}+1\right)\left(\lambda_{2}+\lambda_{3}+2\right)$.

Demonstração. Seja $v_{1}-v_{2}=\lambda_{1}+1 \notin \mathbb{Z}, v_{2}-v_{3}=\lambda_{2}+1 \in \mathbb{Z}_{>0}, v_{3}-v_{4}=\lambda_{3}+1 \in \mathbb{Z}_{>0} \mathrm{e}$ $v_{1}+v_{2}+v_{3}+v_{4}=-6$. Portanto o módulo $L(\lambda)$ pode ser realizado como um módulo de Gelfand-Tselin $V_{\mathcal{C}}(T(v))$ em que, $T(v)$ é a tabela singular

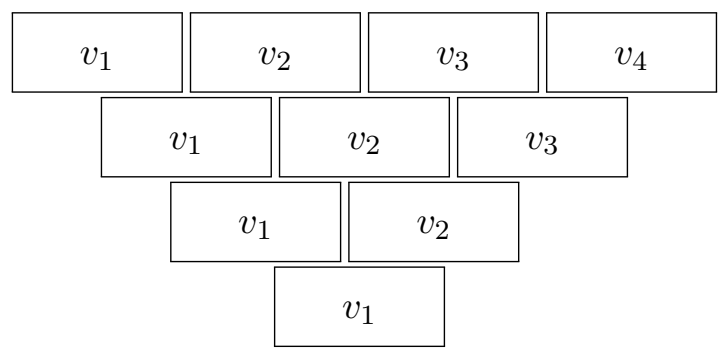

e seja $\mathcal{C}$ o conjunto das relações definidas pelo seguinte grafo:
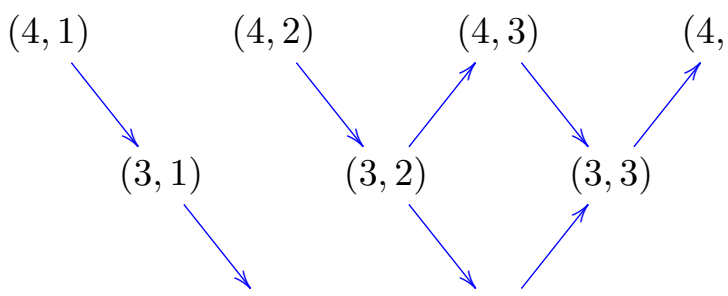

$(3,2)$

$(2,1)$

$(1,1)$

Uma base para este módulo é dada pelo conjunto de tabelas

$$
\mathcal{B}_{\mathcal{C}}(T(v))=L\left(\begin{array}{l}
l \leq m \leq r \leq 0 \\
-\lambda_{2} \leq s \leq 0 \\
-\lambda_{3} \leq t \leq 0 \\
t-\lambda_{2} \leq n \leq s
\end{array}\right) .
$$

Lembramos que se $T(v)$ é um vetor de peso com peso $\lambda=\left(\lambda_{1}, \lambda_{2}, \lambda_{3}\right)$ e $i \in \mathbb{Z}$, então: 


\begin{tabular}{|c|c|}
\hline Tabela & Peso \\
\hline$T(v)$ & $\left(\lambda_{1}, \lambda_{3}, \lambda_{3}\right)$ \\
\hline$E_{21}^{i}(T(v))$ & $\left(\lambda_{1}-2 i, \lambda_{2}+i, \lambda_{3}\right)$ \\
\hline$E_{32}^{i}(T(v))$ & $\left(\lambda_{1}+i, \lambda_{2}-2 i, \lambda_{3}+i\right)$ \\
\hline$E_{43}^{i}(T(v))$ & $\left(\lambda_{1}, \lambda_{2}+i, \lambda_{3}-2 i\right)$ \\
\hline$E_{31}^{i}(T(v))$ & $\left(\lambda_{1}-i, \lambda_{2}-i, \lambda_{3}+i\right.$ \\
\hline$E_{41}^{i}(T(v))$ & $\left(\lambda_{1}-i, \lambda_{2}, \lambda_{3}-i\right)$ \\
\hline$E_{42}^{i}(T(v))$ & $\left(\lambda_{1}+i, \lambda_{2}-i, \lambda_{3}-i\right)$ \\
\hline
\end{tabular}

Além disso, para qualquer $i_{1}, i_{2}, i_{3} \in \mathbb{Z}_{\geq 0}$, o vetor $E_{43}^{i_{3}} E_{32}^{i_{2}} E_{21}^{i_{1}}(T(v))$ é um vetor de peso com peso

$$
\lambda+\left(i_{2}-2 i_{1}, i_{1}+i_{3}-2 i_{2}, i_{2}-2 i_{3}\right)=\left(\lambda_{1}-\frac{a p}{q}-2 i_{1}+i_{2}, \lambda_{2}+i_{1}-2 i_{2}+i_{3}, \lambda_{3}+i_{2}-2 i_{3}\right)
$$

Por outro lado, a ação da subalgebra de Cartan de $\mathfrak{s l}_{4}$ em cada $T(w) \in \mathcal{B}_{\mathcal{C}}(T(v))$ é:

- $h_{1}(T(w))=\left(a_{1}-a_{2}+2 l-(m+n)\right) T(w)=\left(\lambda_{1}-\frac{a p}{q}+2 l-(m+n)\right) T(w)$

- $h_{2}(T(w))=\left(a_{2}-a_{3}+2(m+n)-(l+r+s+t)\right) T(w)=\left(\lambda_{2}+2(m+n)-(l+r+s+t)\right) T(w)$

- $h_{2}(T(w))=\left(a_{3}-a_{4}+2(r+s+t)-(m+n)\right) T(w)=\left(\lambda_{3}+2(r+s+t)-(m+n)\right) T(w)$

Com efeito, $T(w)$ é um vetor de peso com peso

$$
\begin{aligned}
& \lambda+(2 l-(m+n), 2(m+n)-(l+r+s+t), 2(r+s+t)-(m+n)) \\
& =\left(\lambda_{1}-\frac{a p}{q}+2 l-(m+n), \lambda_{2}+2(m+n)-(l+r+s+t), \lambda_{3}+2(r+s+t)-(m+n)\right) .
\end{aligned}
$$

Portanto, todas as tabelas $T(w)$ com o mesmo peso que $E_{43}^{i_{3}} E_{32}^{i_{2}} E_{21}^{i_{1}}(T(v))$ satisfazem o seguinte sistema:

$$
\left\{\begin{array} { r l } 
{ 2 l - ( m + n ) } & { = - 2 i _ { 1 } + i _ { 2 } } \\
{ - l + 2 ( m + n ) - ( r + s + t ) } & { = i _ { 1 } - 2 i _ { 2 } + i _ { 3 } } \\
{ - ( m + n ) + 2 ( r + s + t ) } & { = i _ { 2 } - 2 i _ { 3 } }
\end{array} \Longleftrightarrow \left\{\begin{array}{r}
l=-i_{1} \\
m+n=-i_{2} \\
r+s+t=-i_{3}
\end{array}\right.\right.
$$

Assim, para qualquer $\left(i_{1}, i_{2}, i_{3}\right) \in \mathbb{Z}_{\geq 0}$ fixado, temos uma opção para $l$, uma opção para $m$ para qualquer opção $n$ e uma opção por $r$ para qualquer opção de $(s, t)$. Mas por hipótese, $-\lambda_{2} \leq s \leq 0$, $-\lambda_{3} \leq t \leq 0$ e $-\lambda_{3}-\lambda_{2} \leq n \leq 0$, dado que $t-\lambda_{2} \leq n \leq s$. Portanto, o máximo número de sêxtuplas diferentes $(r, s, t, m, n, l)$ é $\left(\lambda_{2}+1\right)\left(\lambda_{3}+1\right)\left(\frac{\lambda_{2}+\lambda_{3}}{2}+1\right)$. 


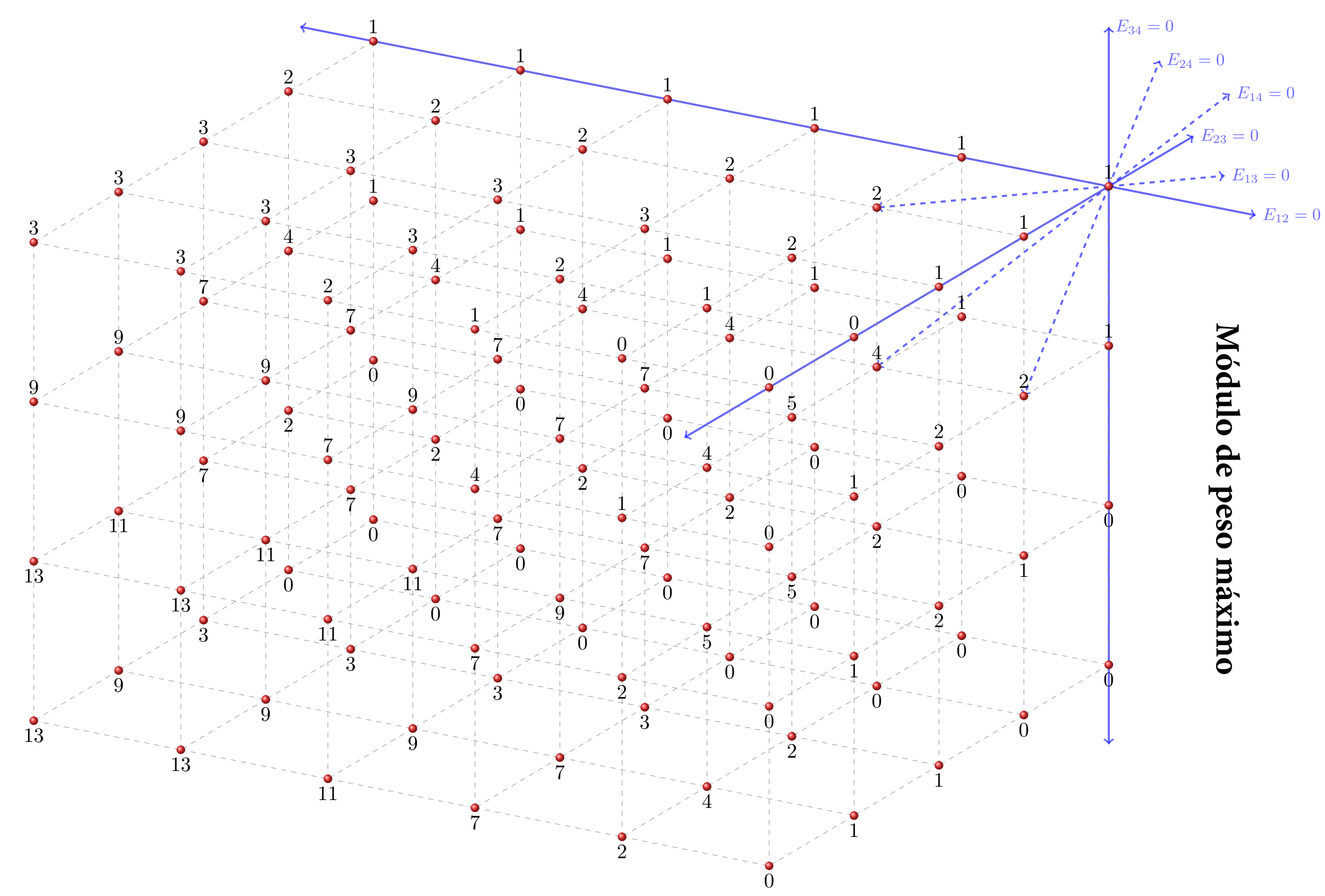


O próximo Lema é uma versão mais geral do Lema 3.8.1 e a prova é semelhante. Mas antes de enunciar o lema, introduziremos a seguinte notação:

A flecha dupla pontilhada seja na posição horizontal como na diagonal entre posições adjacentes da tabela, indicará que as diferenças entre os elementos da tabela em tais posições podem ser inteiras (positivas ou não negativas) ou não. Assim as flechas duplas a seguir

$$
(n, i)<-->(n, i+1) \quad(r, s)
$$

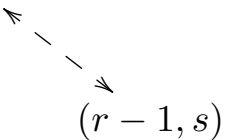

terão as seguinte interpretações possíveis:

a) $v_{n, i}-v_{n, i+1} \notin \mathbb{Z}$ ou $v_{n, i}-v_{n, i+1} \in \mathbb{Z}_{\geq 0}$.

b) $v_{r, s}-v_{r-1, s} \notin \mathbb{Z}$ ou $v_{r, s}-v_{r-1, s} \in \mathbb{Z}_{\geq 0}$ ou $v_{r-1, s}-v_{r, s} \in \mathbb{Z}_{>0}$.

Lema 3.8.2. Seja $\mathcal{C}$ o conjunto de relações com grafo

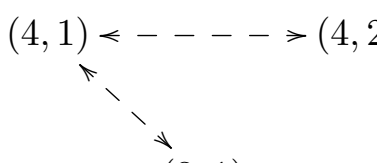

$(3,1)$

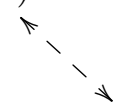

$(2,1)$

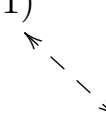

$(1,1)$

$e T(v)$

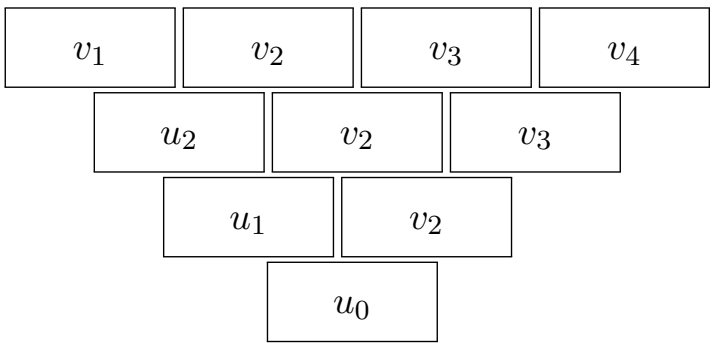

uma $\mathcal{C}$-realização tal que $u_{i}-v_{2} \notin \mathbb{Z}$ para $i=0,1,2$. Então, $V_{\mathcal{C}}(T(v))$ é um módulo limitado de grau $\frac{\left(v_{2}-v_{3}\right)\left(v_{3}-v_{4}\right)\left(v_{2}-v_{4}\right)}{2}$.

Com relação ao Lema anterior fazemos as seguintes observações:

- Pela convenção adotada anteriormente a respeito da flecha dupla pontilhada e pelo Teorema 3.3.19, temos que o módulo $V_{\mathcal{C}}(T(v))$ do Lema 3.8.2 não é necessariamente simples.

- Para $\mathfrak{g l}_{3}$ todos os módulos limitados são realizados em [FGR20] e para $\mathfrak{g l}_{n}$ alguns módulos densos são realizados em [Maz03] (veja a Seção 3).

Em particular, podemos realizar cada classe de $\mathfrak{s l}_{4}$-módulos simples com espaços de peso de dimensão finita (a classificação completa pode ser encontrada em [Mat00]).

A partir de agora e até o final deste capítulo, escolheremos números complexos $\left\{u_{0}, u_{1}, u_{2}\right\}$, tais que $u_{i}-v_{2} \notin \mathbb{Z}$ para qualquer $i=0,1,2$. Ademais, consideremos tabelas $T(v)$ da forma 


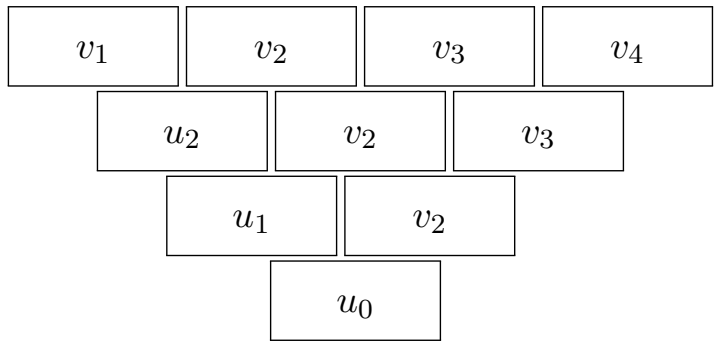

\subsection{1 $\mathfrak{s l}_{4}$-Módulos de $\Gamma_{s t}$-relações que são $\mathfrak{s l}_{2}$-induzidos.}

Suponhamos que $u_{0}-v_{1} \notin \mathbb{Z}$ e $u_{1}=u_{2}=v_{1}$.

Lema 3.8.3. Seja $\mathcal{C}$ o conjunto de relações com grafo

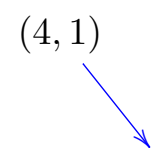

$(3,1)$

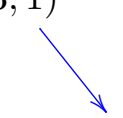

$(2,1)$
$(4,2)$

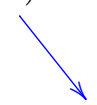

$(3,2)$
$(4,3)$

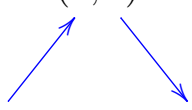

$(3,3)$

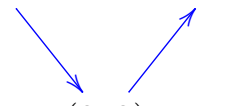

$(2,2)$

$(1,1)$

e $T(v)$ é a tabela singular

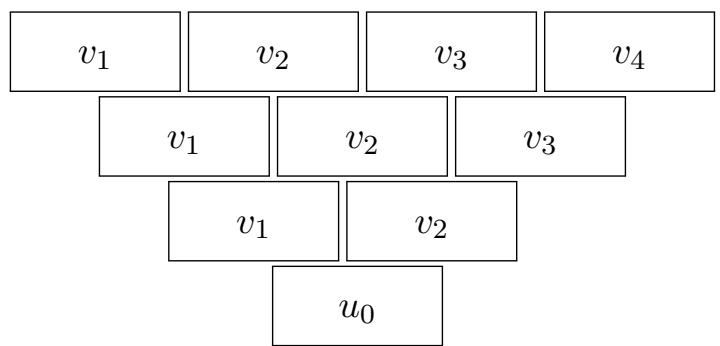

Então $V_{\mathcal{C}}(T(v))$ é um módulo de Gelfand-Tselin, cuja base é o conjunto de tabelas

$$
\mathcal{B}_{\mathcal{C}}(T(v))=L\left(\left(\begin{array}{c}
m \leq r \leq 0 \\
v_{3}-v_{2}<s \leq 0 \\
v_{4}-v_{3}<t \leq 0 \\
t+v_{3}-v_{2}<n \leq s \\
l \in \mathbb{Z}
\end{array}\right) .\right.
$$

Desse maneira, o módulo simples $V_{\mathcal{C}}(T(v))$ é isomorfo ao módulo induzido $L_{\mathfrak{p}}(\lambda, N)$, em que a subálgebra de Levi de $\mathfrak{p}$ é isomorfa com $\mathfrak{s l}_{2} \oplus \mathbb{C} h_{2} \oplus \mathbb{C} h_{3}$ e $N$ é um $\mathfrak{s l}_{2}$-módulo simples cuspidal. 
Observação 3.8.4. No Lema 3.8.3 é importante lembramos que $\mathcal{B}_{\mathcal{C}}(T(v))$ representa o conjunto de tabelas

$\left\{T\left(v+l \delta^{11}+m \delta^{21}+n \delta^{22}+r \delta^{31}+s \delta^{32}+t \delta^{33}\right) \mid m \leq r \leq 0, v_{3}-v_{2}<s \leq 0, v_{4}-v_{3}<t \leq 0, t+v_{3}-v_{2}<n \leq s, l \in \mathbb{Z}\right\}$.

Demonstração. No Teorema 3.3.19, temos que $V_{\mathcal{C}}(T(v))$ é um $\mathfrak{s l}_{4}$-módulo simples. Definimos $N$ como o espaço vetorial gerado pelo conjunto de tabelas $\left\{T\left(v+l \delta^{11}\right) \mid l \in \mathbb{Z}\right\}$ e $\mathfrak{s l}_{2}$ a álgebra de Lie simples gerada por $E_{12}, E_{21}$. Pela aplicação direta das fórmulas de Gelfand-Tsetlin, temos que $E_{23}, E_{34}$ e $E_{13}$ (consequentemente, $E_{24}$ e $E_{14}$ ) agem trivialmente em $N$. Ademais, $N$ é isomorfo ao módulo de Gelfand-Tseltin fortemente genérico $V_{\emptyset}\left(T\left(v^{\prime}\right)\right)$, em que $T\left(v^{\prime}\right)$ é a tabela

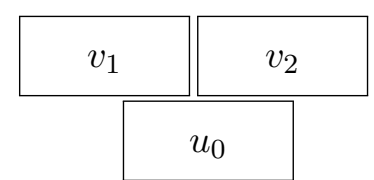

com base o conjunto de tabelas $\mathcal{B}_{\emptyset}\left(T\left(v^{\prime}\right)\right)=\left\{T\left(v^{\prime}+l\right) \mid l \in \mathbb{Z}\right\}$. Em que $T\left(v^{\prime}+l\right)$ é a tabela

\begin{tabular}{|c|c|}
\hline$v_{1}$ & $v_{2}$ \\
\hline$u_{0}+l$
\end{tabular}

Segue do Teorema 3.3.19 que $V_{\emptyset}\left(T\left(v^{\prime}\right)\right)$ é simples e uma vez que $E_{12}$ e $E_{21}$ agem injetivamente em $N$ (veja Proposição 1.4.3), concluímos que $N$ é um $\mathfrak{s l}_{2}$-módulo cuspidal. 


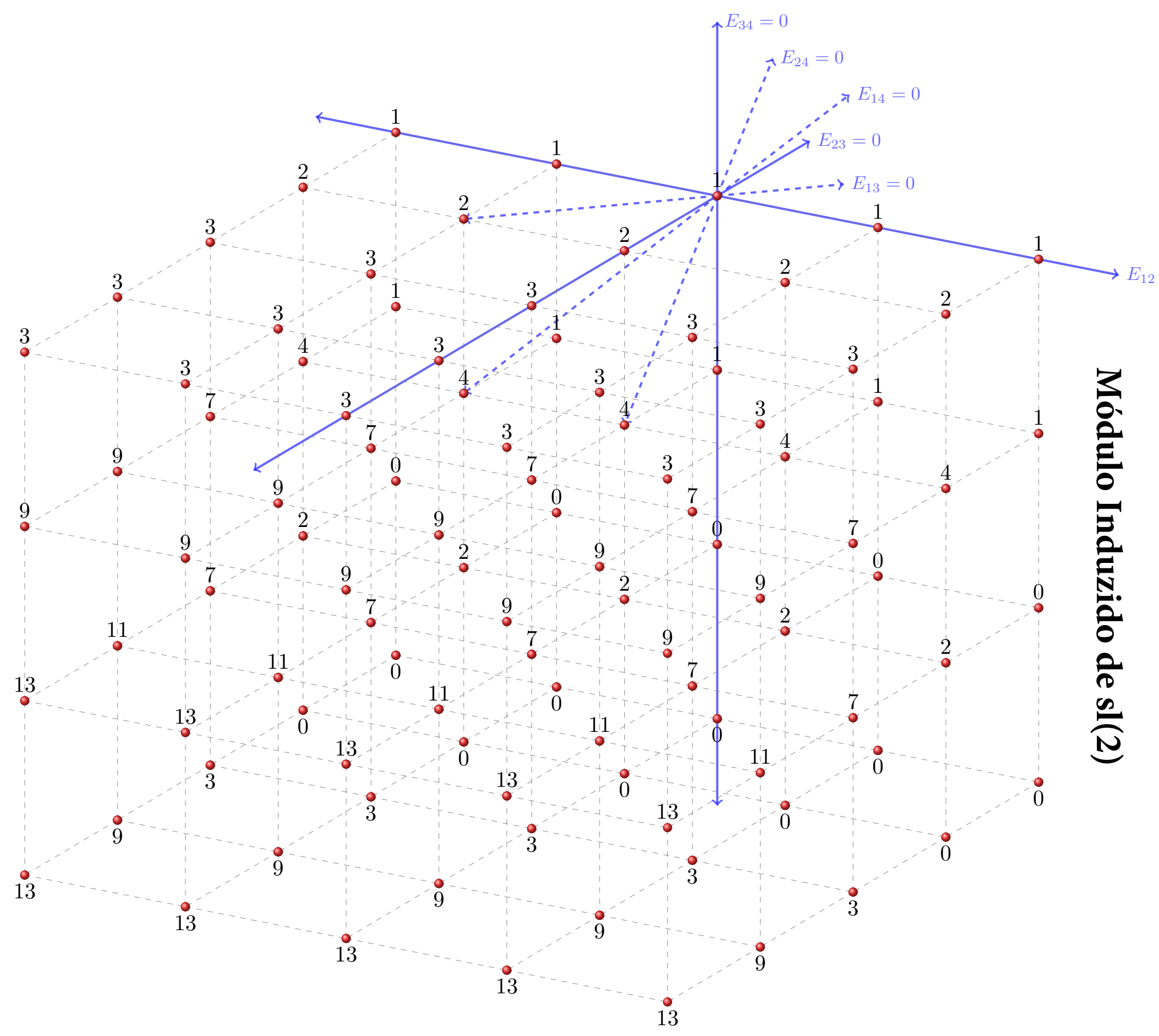




\subsection{2 $\mathfrak{s l}_{4}$-Módulos de $\Gamma_{s t}$-relações que são $\mathfrak{s l}_{3}$-induzidos.}

Suponhamos que $u_{0}-u_{1} \notin \mathbb{Z}, u_{1}-v_{1} \notin \mathbb{Z}$ e $u_{2}=v_{1}$.

Lema 3.8.5. Seja $\mathcal{C}$ o conjunto de relações com grafo

$(4,1)$

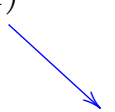

$(3,1)$
$(4,2)$

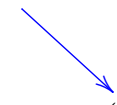

$(3,2)$
$(4,3)$

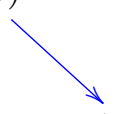

$(3,3)$

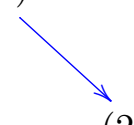

$(2,2)$

e $T(v)$ é a tabela singular

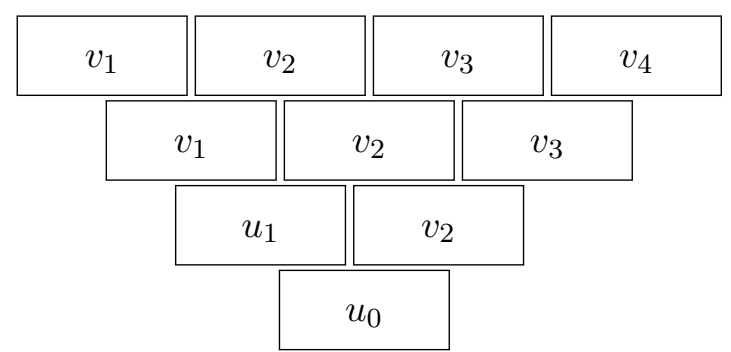

Então $V_{\mathcal{C}}(T(v))$ é um módulo simples de Gelfand-Tselin, cuja base é o conjunto de tabelas

$$
\mathcal{B}_{\mathcal{C}}(T(v))=L\left(\begin{array}{c}
r \leq 0 \\
v_{3}-v_{2}<s \leq 0 \\
v_{4}-v_{3}<t \leq 0 \\
t+v_{3}-v_{2}<n \leq s \\
l, m \in \mathbb{Z}
\end{array}\right) .
$$

Logo, o módulo simples $V_{\mathcal{C}}(T(v))$ é isomorfo ao módulo induzido $L_{\mathfrak{p}}(\lambda, N)$, tal que a subálgebra de Levi de $\mathfrak{p}$ é isomorfa com $\mathfrak{s l}_{3} \oplus \mathbb{C h}_{3}$ e $N$ é um $\mathfrak{s l}_{3}$-módulo simples cuspidal.

Demonstração. No Teorema 3.3.19, temos que $V_{\mathcal{C}}(T(v))$ é um $\mathfrak{s l}_{4}$-módulo simples. Definimos $N$ como o espaço vetorial gerado pelo conjunto de tabelas $\left\{T\left(v+l \delta^{11}+m \delta^{21}+n \delta^{22}\right) \mid l, m \in\right.$ $\mathbb{Z}$ e $\left.v_{3}-v_{2}<n \leq 0\right\}$ e $\mathfrak{s l}_{3}$ a álgebra de Lie simples gerada por $\left\{E_{12}, E_{23}, E_{21}, E_{32}\right\}$. Pela aplicação direta das fórmulas de Gelfand-Tsetlin, temos que $E_{34}, E_{24}$ e $E_{14}$ agem trivialmente em $N$. Como $N$ é isomorfo ao módulo genérico de Gelfand-Tseltin $V_{\mathcal{C}^{\prime}}\left(T\left(v^{\prime}\right)\right)$, em que $T\left(v^{\prime}\right)$ é a tabela

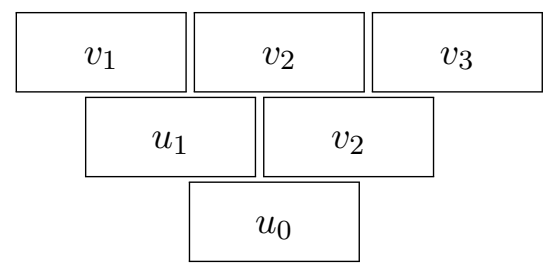


e $\mathcal{C}^{\prime}$ é o conjunto de relações definidas pelo seguinte grafo:

$(1,1)$

Com base no conjunto de tabelas $\mathcal{B}_{\mathcal{C}^{\prime}}\left(T\left(v^{\prime}\right)\right)=\left\{T\left(v^{\prime}+l \delta^{11}+m \delta^{21}\right) \mid l, m \in \mathbb{Z}, v_{3}-v_{2}<n \leq 0\right\}$, em que $T\left(v^{\prime}+l \delta^{11}+m \delta^{21}\right)$ representa a tabela

\begin{tabular}{|c|c|c|}
\hline$v_{1}$ & \multicolumn{1}{|c|}{$v_{2}$} & $v_{3}$ \\
\hline$u_{1}+m$ & $v_{2}$ \\
\hline
\end{tabular}

Por fim, o módulo $V_{\mathcal{C}^{\prime}}\left(T\left(v^{\prime}\right)\right)$ é o $\mathfrak{s l}_{3}$-módulo simples cuspidal $L_{2}$ com espaços de peso de dimensão $v_{2}-v_{3}$ no bloco genérico (G8) (veja [FGR20], seção 7.2). 


\subsection{3 $\mathfrak{s l}_{4}$-Módulos cuspidais de $\Gamma_{s t}$-relações.}

Suponhamos que $u_{0}-u_{1} \notin \mathbb{Z}, u_{1}-u_{2} \notin \mathbb{Z}$ e $u_{2}-v_{1} \notin \mathbb{Z}$.

Lema 3.8.6. Seja $\mathcal{C}$ o conjunto de relações com grafo

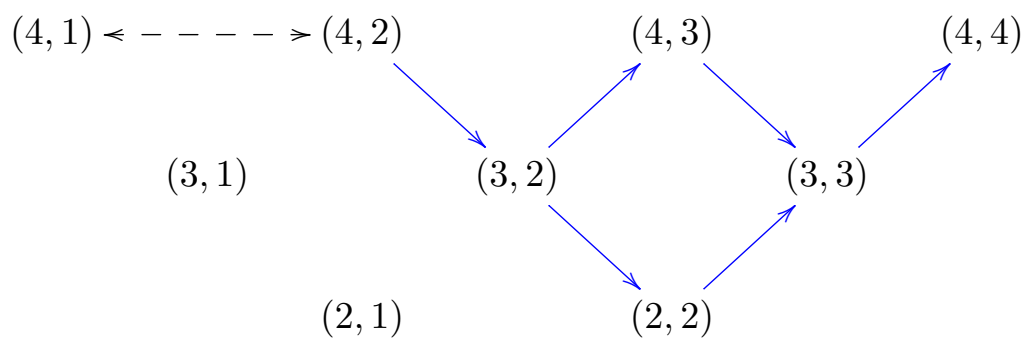

$(1,1)$

e $T(v)$ seja a tabela singular

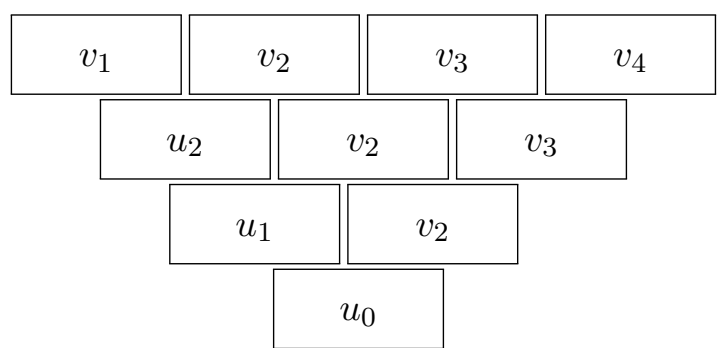

Então $V_{\mathcal{C}}(T(v))$ é um módulo simples de Gelfand-Tselin, cuja base é o conjunto de tabelas

$$
\mathcal{B}_{\mathcal{C}}(T(v))=L\left(\begin{array}{c}
v_{3}-v_{2}<s \leq 0 \\
v_{4}-v_{3}<t \leq 0 \\
t+v_{3}-v_{2}<n \leq s \\
l, m, r \in \mathbb{Z}
\end{array}\right) .
$$

Então, $V_{\mathcal{C}}(T(v))$ é um $\mathfrak{s l}_{4}$-módulo simples cuspidal.

Demonstração. No Teorema 3.3.19, temos que $V_{\mathcal{C}}(T(v))$ é um $\mathfrak{s l}_{4}$-módulo simples. Agora, a partir das fórmulas de Gelfand-Tsetlin, temos o seguinte:

- $H_{1}(T(v))=\lambda_{1} T(v)$, em que $\lambda_{1}=2 u_{0}-u_{1}-v_{2}-1$

- $H_{2}(T(v))=\lambda_{2} T(v)$, em que $\lambda_{2}=2 u_{1}-u_{2}-u_{0}+v_{2}-v_{3}-1$

- $H_{3}(T(v))=\lambda_{3} T(v)$, em que $\lambda_{3}=2 u_{2}-u_{1}-v_{1}+v_{3}-v_{4}-1$

Definimos a transformação linear $\lambda: \mathfrak{h}^{*} \longrightarrow \mathbb{C}, \lambda\left(h_{i}\right)=\lambda_{i}$ para cada $i=1,2,3$. Além disso, lembramos que as transformações lineares $\alpha_{i}: \mathfrak{h}^{*} \longrightarrow \mathbb{C}$ para cada $i=1,2,3$ estão definidas

- $\alpha_{1}\left(h_{1}\right)=2, \alpha_{1}\left(h_{1}\right)=-1$ e $\alpha_{1}\left(h_{3}\right)=0$;

- $\alpha_{2}\left(h_{1}\right)=-1, \alpha_{2}\left(h_{1}\right)=2$ e $\alpha_{2}\left(h_{3}\right)=-1$; 
- $\alpha_{3}\left(h_{1}\right)=0, \alpha_{3}\left(h_{1}\right)=-1$ e $\alpha_{3}\left(h_{3}\right)=2$.

Assim, para todo $(i, j, k) \in \mathbb{Z}^{3}$, temos a transformação linear $\lambda+i \alpha_{1}+j \alpha_{2}+k \alpha_{3}: \mathfrak{h}^{*} \longrightarrow \mathbb{C}$, de modo que:

- $\left(\lambda+i \alpha_{1}+j \alpha_{2}+k \alpha_{3}\right)\left(h_{1}\right)=\lambda_{1}+2 i-j$;

- $\left(\lambda+i \alpha_{1}+j \alpha_{2}+k \alpha_{3}\right)\left(h_{2}\right)=\lambda_{2}-i+2 j-k$;

- $\left(\lambda+i \alpha_{1}+j \alpha_{2}+k \alpha_{3}\right)\left(h_{3}\right)=\lambda_{3}-j+2 k$.

Definimos $T(w)=T\left(v+i \delta^{11}+j \delta^{21}+k \delta^{31}\right)$

\begin{tabular}{|c|c|c|c|}
\hline$v_{1}$ & $v_{2}$ & $v_{3}$ & $v_{4}$ \\
\hline$u_{2}+k$ & $v_{2}$ & $v_{3}$ \\
\hline$u_{1}+j$ & $v_{2}$ \\
\hline
\end{tabular}

Observemos que $T(w)=T\left(v+i \delta^{11}+j \delta^{21}+k \delta^{31}\right) \in \mathcal{B}_{\mathcal{C}}(T(v))$, em particular $T(w) \neq 0$ em $V_{\mathcal{C}}(T(v))$.

A ação da subalgebra de Cartan em $T(w)$ é (veja Exemplo 3.4.3):

- $H_{1}(T(w))=\left(\lambda_{1}+2 i-j\right) T(w)$

- $H_{2}(T(w))=\left(\lambda_{2}-i+2 j-k\right) T(w)$;

- $H_{3}(T(w))=\left(\lambda_{3}-j+2 k\right) T(w)$.

Isto prova que o suporte a $V_{\mathcal{C}}(T(v))$ é exatamente o $Q$-coset de $\lambda$ e $V_{\mathcal{C}}(T(v))$ é um $\mathfrak{s l}_{4}$-módulo cuspidal (veja Proposição 1.4.3). 


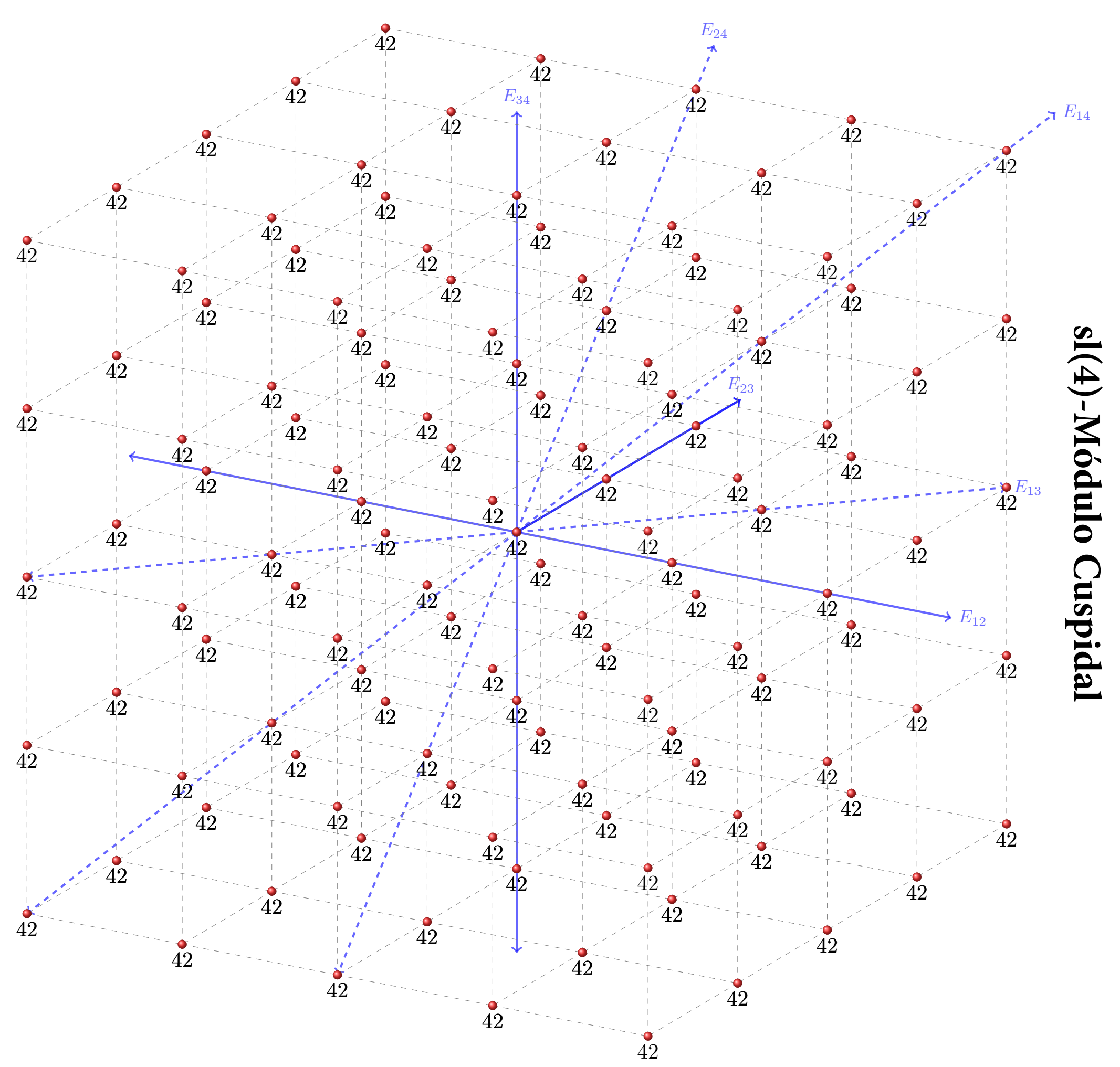




\subsection{4 $\mathfrak{s l}_{4}$-Módulos de $\Gamma_{s t}$-relações $\mathfrak{s l}_{2} \oplus \mathfrak{s l}_{2}$-induzidos.}

Suponhamos que $v_{i}-v_{j} \notin \mathbb{Z}$ para todo $1 \leq i<j \leq 4, u_{0}-v_{i} \notin \mathbb{Z}$ para cada $i=1,2$ e $u_{1}-v_{i} \notin \mathbb{Z}$ para cada $i=1,2,3,4$.

Lema 3.8.7. Seja $\mathcal{C}$ o conjunto de relações com grafo

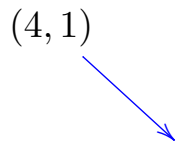

$(3,1)$

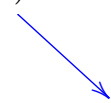

$(2,1)$

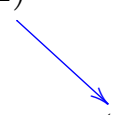

$(2,2)$

e $T(v)$ seja a tabela genérica

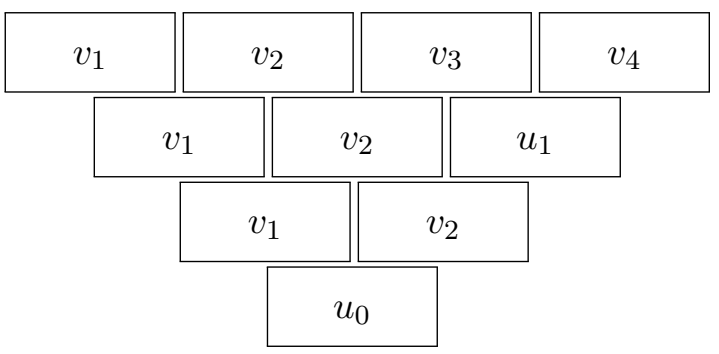

Então $V_{\mathcal{C}}(T(v))$ é um módulo simples de Gelfand-Tselin, cuja base é o conjunto de tabelas

$$
\mathcal{B}_{\mathcal{C}}(T(v))=L\left(\left\{\begin{array}{l}
m \leq r \leq 0 \\
n \leq s \leq 0 \\
l, t \in \mathbb{Z}
\end{array}\right) .\right.
$$

Assim, o módulo simples não limitado $V_{\mathcal{C}}(T(v))$ é isomorfo ao módulo induzido $L_{\mathfrak{p}}(\lambda, N)$, em que $\mathfrak{p}$ tem a subálgebra de Levi isomorfa a $\mathfrak{s l}_{2} \oplus \mathfrak{s l}_{2} \oplus \mathbb{C h}_{2}$ e $N$ é um $\mathfrak{s l}_{2} \oplus \mathfrak{s l}_{2}$-módulo denso simples.

Observação 3.8.8. É importante notar que o módulo construído no Lema 3.8.7 não é limitado. Ressaltamos também que basta supor que $v_{i}-v_{j} \notin \mathbb{Z}$ para todo $1 \leq i<j \leq 3$. Por outro lado, observamos que o grafo associado ao conjunto admissível $\mathcal{C}$ não aparece na lista apresentada no apêndice, pois a tabela $T(v)$ é fortemente genérica. Ressaltamos ainda que cada um dos espaços de peso do módulo $V_{\mathcal{C}}(T(v))$ tem dimensão finita, mais ainda as dimensões formam a sequência tetraedral, isto é, $1,4,10,20,35,56,84,120,165, \ldots$

$\mathrm{O}$ reticulado de peso do módulo $V_{\mathcal{C}}(T(v))$ é: 


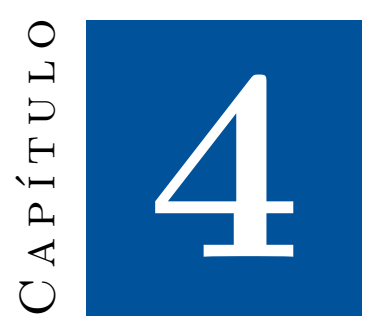

\section{Módulos admissíveis para $\mathfrak{s l}_{n+1}$}

Neste capítulo daremos uma realização por tabelas para $\mathfrak{s l}_{n+1}$-módulos simples admissíveis de peso máximo e induzidos de $\mathfrak{s l}_{2}$ nas órbitas minimal e principal. Usando a classificação de $\mathfrak{s l}_{n+1}$-módulos simples de relações de peso máximo (veja Teorema 3.4.9) e induzidos de $\mathfrak{s l}_{2}$ (veja Teorema 3.5.9), onde são dadas a base explícita e a ação dos geradores do $\mathfrak{s l}_{n+1}$, somos capazes de apresentar explicitamente todos os módulos simples (de peso máximo e induzidos de $\mathfrak{s l}_{2}$ ) de Gelfand-Tsetlin com o anulador necessário para pertencerem a cada uma destas órbitas. Lembrando que pelo Corolário 3.4.8, temos que todo módulo simples de peso máximo admissível é um módulo de relações. Ainda, veremos que todos os módulos de peso máximo simples na órbita nilpotente minimal são módulos limitados (veja Corolário 3.4.10). Entretanto, todos os módulos de peso máximo simples na órbita nilpotente principal são módulos de Verma (veja Corolário 3.4.12). Consequentemente, todos os $\mathfrak{s l}_{n+1}$-módulos simples induzidos de $\mathfrak{s l}_{2}$ na órbita nilpotente minimal são módulos limitados de relações, enquanto que todos os $\mathfrak{s l}_{n+1}$-módulos simples induzidos de $\mathfrak{s l}_{2}$ na órbita nilpotente principal são módulos de relações ilimitados.

Lembramos que um número admissível $k$ para $\widehat{\mathfrak{s l}}_{n+1}$ com denominador $q \in \mathbb{N}$ é definido por $k=\frac{p}{q}-n-1, \quad p>n, q \geq 1$ e $(p, q)=1$.

\subsection{Módulos simples na órbita nilpotente minimal para $\mathfrak{s l}_{n+1}$}

Nesta seção, discutiremos a construção explícita dos g-módulos simples de peso máximo admissíveis e dos $\mathfrak{g}$-módulos induzidos de $\mathfrak{s l}_{2}$ na órbita nilpotente minimal para $\mathfrak{g}=\mathfrak{s l}_{n+1}$. 


\section{Órbita nilpotente minimal}

Seguindo Frenkel-Malikov e Arakawa (veja detalhes em [Ara17, AFR17, FZ92]), para descrever $\mathfrak{g}$-módulos simples admissíveis na órbita minimal $\mathbb{O}_{\text {min }}$ do nível $k$ precisamos encontrar os $\mathfrak{g}$-módulos simples $V$ para os quais $\operatorname{Ann}_{U(\mathfrak{g})} V=\operatorname{Ann} L_{\lambda}$, para cada $\lambda \in\left[\overline{\operatorname{Pr}}_{k}^{\mathbb{O}_{\text {min }}}\right]$. Começamos com os módulos de peso máximo.

Lembramos que $\mathbb{O}_{\text {min }}$ indica a órbita nilpotente minimal de $\mathfrak{g}$ e $\operatorname{dim} \mathbb{O}_{\text {min }}=2 n$ (veja [Wan99]). Mais ainda, considerando (2.24), um elemento de $\left[\overline{\operatorname{Pr}}_{k}^{\mathbb{O}_{\text {min }}}\right]$ tem o formato

$$
\Lambda=\bar{\lambda}-\frac{a p}{q} \varpi_{1}=\left(\lambda_{1}-\frac{a p}{q}, \lambda_{2}, \lambda_{3}, \ldots, \lambda_{n-1}, \lambda_{n}\right)
$$

em que $\lambda_{i} \in \mathbb{Z}_{\geq 0}$, para todo $i=1, \ldots, n$ são tais que $\lambda_{1}+\ldots+\lambda_{n}<p-n$ e $a \in\{1,2, \ldots, q-1\}$.

\subsubsection{Realização explícita dos módulos de peso máximo}

O objetivo desta seção é provar que todos os $\mathfrak{s l}_{n+1}$-módulos de peso máximo simples são limitados e podem ser realizados como $V_{\mathcal{C}}(T(L))$ para algum conjunto admissível de relações $\mathcal{C}$. Neste ponto é importante levar em consideração o fato que, dado um peso admissível na órbita nilpotente minimal, nem todo peso conjugado por um elemento do grupo de Weyl é um peso admissível e, portanto o módulo de peso máximo nem sempre é um módulo de relações. Por outro lado, existem módulos de peso máximo que são módulos de relações, mas não são admissíveis uma vez que o anulador do módulo é diferente do anulador de um módulo admissível (veja exemplos 4.1.1 e 4.1.2).

Exemplo 4.1.1. Seja $\mathfrak{g}=\mathfrak{s l}_{3}$. Por (2.24), os elementos de $\left[\overline{\operatorname{Pr}}_{k}^{\mathbb{O}_{\text {min }}}\right]$ são representados por $\mathfrak{s l}_{3}$-pesos da forma $\Lambda_{1}=\left(\lambda_{1}-\frac{a p}{q}, \lambda_{2}\right)$ e como estes são dominantes regulares, pelo Corolário 1.3.18, os módulos de peso máximo admissíveis simples na órbita minimal são $L\left(\Lambda_{i}\right), i=1,2,3$, em que:

- $\Lambda_{2}=s_{1} \cdot \Lambda_{1}=\left(\frac{a p}{q}-\lambda_{1}-2, \lambda_{1}+\lambda_{2}-\frac{a p}{q}+1\right)$;

- $\Lambda_{3}=s_{2} s_{1} \cdot \Lambda_{1}=\left(\lambda_{2}, \frac{a p}{q}-\lambda_{1}-\lambda_{2}-3\right)$.

Esses módulos têm multiplicidades de peso limitadas por $\lambda_{2}+1$.

Por outro lado, consideremos os pesos:

- $\Lambda_{4}=s_{2} \cdot \Lambda_{1}=\left(\lambda_{1}+\lambda_{2}-\frac{a p}{q}+1,-\lambda_{2}-2\right)$;

- $\Lambda_{5}=s_{1} s_{2} \cdot \Lambda_{1}=\left(\frac{a p}{q}-\lambda_{1}-\lambda_{2}-3, \lambda_{1}-\frac{a p}{q}\right)$;

- $\Lambda_{6}=s_{1} s_{2} s_{1} \cdot \Lambda_{1}=\left(-\lambda_{2}-2, \frac{a p}{q}-\lambda_{1}-2\right)$. 
Dado que $\lambda_{2} \in \mathbb{Z}_{\geq 0}$, então $\left\langle\Lambda_{4}+\rho, \alpha_{2}^{\vee}\right\rangle \in \mathbb{Z}_{<0},\left\langle\Lambda_{5}+\rho, \alpha_{12}^{\vee}\right\rangle \in \mathbb{Z}_{<0}$ e $\left\langle\Lambda_{6}+\rho, \alpha_{1}^{\vee}\right\rangle \in \mathbb{Z}_{<0}$. Assim, para cada $i=4,5,6$ o peso $\Lambda_{i}$ não é dominante, em particular $\Lambda_{i} \notin \overline{P r_{k}}$ e $A n n_{U\left(\mathfrak{s l}_{3}\right)} L\left(\Lambda_{i}\right) \neq$ $A n n_{U\left(\mathfrak{s l}_{3}\right)} L\left(\Lambda_{1}\right)$ pelo Teorema 2.4.5. Portanto, os únicos $\mathfrak{s l}_{3}$-módulos de peso máximo na órbita minimal são $L\left(\Lambda_{i}\right)$ para $i=1,2,3$. Por outro lado, $\left\langle\Lambda_{4}+\rho, \alpha_{1}^{\vee}\right\rangle=\lambda_{1}+\lambda_{2}-\frac{a p}{q}+2 \notin \mathbb{Z}$ e $\left\langle\Lambda_{4}+\rho, \alpha_{12}^{\vee}\right\rangle=\lambda_{1}-\frac{a p}{q}+1 \notin \mathbb{Z}$, então $\Lambda_{4}$ é um peso antidominante. Analogamente é possível provar que $\Lambda_{5}$ e $\Lambda_{6}$ são pesos antidominantes. Assim, pelo Teorema 1.3.14 concluímos que:

$$
A n n_{U\left(\mathfrak{s l}_{3}\right)} L\left(\Lambda_{4}\right)=A n n_{U\left(\mathfrak{s l}_{3}\right)} L\left(\Lambda_{5}\right)=A n n_{U\left(\mathfrak{s l}_{3}\right)} L\left(\Lambda_{6}\right)=U(\mathfrak{g}) \operatorname{ker} \chi_{\Lambda_{1}}
$$

Finalmente, é importante observar que $L\left(\Lambda_{4}\right)$ e $L\left(\Lambda_{5}\right)$ são módulos de $\Gamma$-relações, pois estes são módulos genéricos. Entretanto, $L\left(\Lambda_{6}\right)$ não é um módulo de $\Gamma$-relações.

Exemplo 4.1.2. Seja $\mathfrak{g}=\mathfrak{s l}_{4}$. Devido a (2.24), os elementos de $\left[\overline{\operatorname{Pr}}_{k}^{\mathbb{O}_{\text {min }}}\right]$ são dominantes regulares uma vez que estes $\mathfrak{s l}_{4}$-pesos são da forma $\Lambda_{1}=\left(\lambda_{1}-\frac{a p}{q}, \lambda_{2}, \lambda_{3}\right)$, logo a órbita de Weyl de $\Lambda_{1}$ é:

- $\Lambda_{2}=s_{1} \cdot \Lambda_{1}=\left(\frac{a p}{q}-\lambda_{1}-2, \lambda_{1}-\frac{a p}{q}+\lambda_{2}+1, \lambda_{3}\right)$;

- $\Lambda_{3}=s_{2} s_{1} \cdot \Lambda_{1}=\left(\lambda_{2}, \frac{a p}{q}-\lambda_{1}-\lambda_{2}-3, \lambda_{1}+\lambda_{2}+\lambda_{3}-\frac{a p}{q}+2\right)$;

- $\Lambda_{4}=s_{3} s_{2} s_{1} \cdot \Lambda_{1}=\left(\lambda_{2}, \lambda_{3}, \frac{a p}{q}-\lambda_{1}-\lambda_{2}-\lambda_{3}-4\right)$;

- $\Lambda_{5}=s_{2} \cdot \Lambda_{1}=\left(\lambda_{1}+\lambda_{2}-\frac{a p}{q}+1,-\lambda_{2}-2, \lambda_{2}+\lambda_{3}+1\right)$;

- $\Lambda_{6}=s_{3} \cdot \Lambda_{1}=\left(\lambda_{1}-\frac{a p}{q}, \lambda_{2}+\lambda_{3}+1,-\lambda_{3}-2\right)$;

- $\Lambda_{7}=s_{1} s_{2} \cdot \Lambda_{1}=\left(\frac{a p}{q}-\lambda_{1}-\lambda_{2}-3, \lambda_{1}-\frac{a p}{q}, \lambda_{2}+\lambda_{3}+1\right)$;

- $\Lambda_{8}=s_{1} s_{3} \cdot \Lambda_{1}=\left(\frac{a p}{q}-\lambda_{1}-2, \lambda_{1}+\lambda_{2}+\lambda_{3}-\frac{a p}{q}+2,-\lambda_{3}-2\right)$;

- $\Lambda_{9}=s_{2} s_{3} \cdot \Lambda_{1}=\left(\lambda_{1}+\lambda_{2}+\lambda_{3}-\frac{a p}{q}+2,-\lambda_{2}-\lambda_{3}-3, \lambda_{2}\right)$;

- $\Lambda_{10}=s_{3} s_{2} \cdot \Lambda_{1}=\left(\lambda_{1}+\lambda_{2}-\frac{a p}{q}+1, \lambda_{3},-\lambda_{2}-\lambda_{3}-3\right)$;

- $\Lambda_{11}=s_{1} s_{2} s_{3} \cdot \Lambda_{1}=\left(\frac{a p}{q}-\lambda_{1}-\lambda_{2}-\lambda_{3}-4, \lambda_{1}-\frac{a p}{q}, \lambda_{2}\right)$;

- $\Lambda_{12}=s_{1} s_{3} s_{2} \cdot \Lambda_{1}=\left(\frac{a p}{q}-\lambda_{1}-\lambda_{2}-3, \lambda_{2}+\lambda_{2}+\lambda_{3}+2,-\lambda_{2}-\lambda_{3}-3\right)$;

- $\Lambda_{13}=s_{2} s_{1} s_{3} \cdot \Lambda_{1}=\left(\lambda_{2}+\lambda_{3}+1, \frac{a p}{q}-\lambda_{1}-\lambda_{2}-\lambda_{3}-4, \lambda_{1}+\lambda_{2}-\frac{a p}{q}+1\right)$;

- $\Lambda_{14}=s_{2} s_{1} s_{3} s_{2} \cdot \Lambda_{1}=\left(\lambda_{3}, \frac{a p}{q}-\lambda_{1}-\lambda_{2}-\lambda_{3}-4, \lambda_{1}-\frac{a p}{q}\right)$;

- $\Lambda_{15}=s_{1} s_{2} s_{1} \cdot \Lambda_{1}=\left(-\lambda_{2}-2, \frac{a p}{q}-\lambda_{1}-2, \lambda_{1}-\frac{a p}{q}+\lambda_{2}+\lambda_{3}+2\right)$;

- $\Lambda_{16}=s_{3} s_{1} s_{2} s_{1} \cdot \Lambda_{1}=\left(-\lambda_{2}-2, \lambda_{2}+\lambda_{3}+1, \frac{a p}{q}-\lambda_{1}-\lambda_{2}-\lambda_{3}-4\right)$;

- $\Lambda_{17}=s_{2} s_{1} s_{2} s_{3} \cdot \Lambda_{1}=\left(-\lambda_{2}-\lambda_{3}-3, \frac{a p}{q}-\lambda_{1}-2, \lambda_{1}+\lambda_{2}-\frac{a p}{q}+1\right)$;

- $\Lambda_{18}=s_{3} s_{2} s_{1} s_{3} \cdot \Lambda_{1}=\left(\lambda_{2}+\lambda_{3}+1,-\lambda_{3}-2, \frac{a p}{q}-\lambda_{1}-\lambda_{2}-3\right)$;

- $\Lambda_{19}=s_{2} s_{3} s_{1} s_{2} s_{1} \cdot \Lambda_{1}=\left(\lambda_{2},-\lambda_{2}-\lambda_{3}-3, \frac{a p}{q}-\lambda_{1}-2\right)$;

- $\Lambda_{20}=s_{3} s_{1} s_{2} s_{1} s_{3} \cdot \Lambda_{1}=\left(-\lambda_{2}-\lambda_{3}-3, \lambda_{2}, \frac{a p}{q}-\lambda_{1}-\lambda_{2}-3\right)$;

- $\Lambda_{21}=s_{2} s_{3} s_{2} \cdot \Lambda_{1}=\left(\lambda_{1}+\lambda_{2}+\lambda_{3}-\frac{a p}{q}+2,-\lambda_{3}-2,-\lambda_{2}-2\right)$; 
- $\Lambda_{22}=s_{3} s_{1} s_{2} s_{3} \cdot \Lambda_{1}=\left(\frac{a p}{q}-\lambda_{1}-\lambda_{2}-\lambda_{3}-4, \lambda_{1}+\lambda_{2}-\frac{a p}{q}+1,-\lambda_{2}-2\right)$;

- $\Lambda_{23}=s_{2} s_{3} s_{1} s_{2} s_{3} \cdot \Lambda_{1}=\left(-\lambda_{3}-2, \frac{a p}{q}-\lambda_{1}-\lambda_{2}-3, \lambda_{1}-\frac{a p}{q}\right)$;

- $\Lambda_{24}=s_{1} s_{2} s_{3} s_{1} s_{2} s_{1} \cdot \Lambda_{1}=\left(-\lambda_{2}-2,-\lambda_{3}-2, \frac{a p}{q}-\lambda_{1}-2\right)$;

Observamos que para cada $i=1,2,3,4, \Lambda_{i}$ é dominante regular e não é antidominante. Além do mais, uma das condições no Corolário 3.4.10 é atendida. Portanto, pelo Corolário 1.3.18, os módulos de peso máximo admissíveis na órbita minimal são $L\left(\Lambda_{i}\right), i=1,2,3,4$.

Por outro lado, considerando que $\lambda_{2} \in \mathbb{Z}_{\geq 0}$ e $\lambda_{3} \in \mathbb{Z}_{\geq 0}$ e em seguida para todo $\mu=w \cdot \Lambda_{1}$, em que $w \in W \backslash\left\{1, s_{1}, s_{2} s_{1}, s_{3} s_{2} s_{1}\right\}$ existem $\alpha_{i j} \in \Delta^{+}$de forma que $\left\langle\mu+\rho, \alpha_{i j}^{\vee}\right\rangle \in \mathbb{Z}_{<0}$. Desse modo, $\mu$ não é peso dominante, consequentemente $\mu \notin \overline{P r_{k}}$. Portanto, para cada $i=1,2,3,4$, temos o seguinte: $L\left(\Lambda_{i}\right)$ é um módulo de peso máximo limitado admissível na órbita minimal e para todo $\mu \neq \Lambda_{i}$, o módulo de peso máximo $L(\mu)$ não é admissível.

No entanto, para cada $i \in\{5, \ldots, 24\}$ o peso $\Lambda_{i}$ é regular e nenhuma das condições no Corolário 3.4.10 é satisfeita. Mais explicitamente, temos a seguinte classificação:

a) Para $5 \leq i \leq 14, \Lambda_{i}$ não é antidominante e uma das condições no Teorema 3.4.9 é satisfeita. Portanto, $L\left(\Lambda_{i}\right)$ é um $\Gamma$-módulo de relações com espaços de peso de dimensão ilimitada.

b) Para $15 \leq i \leq 20, \Lambda_{i}$ não é antidominante e nenhuma das condições do Teorema 3.4.9 é satisfeita. Portanto, $L\left(\Lambda_{i}\right)$ não é um $\Gamma$-módulo de relações e as dimensões dos espaços de peso são ilimitadas.

c) Para $21 \leq i \leq 24, \Lambda_{i}$ é antidominante e nenhuma das condições do Teorema 3.4.9 é satisfeita. Portanto, o módulo de Verma $M\left(\Lambda_{i}\right)$ é um módulo simples com dimensões dos espaços de peso ilimitadas, mas não é um $\Gamma$-módulo de relações. Ademais, $A n n_{U\left(\mathfrak{s}_{4}\right)} L\left(\Lambda_{i}\right)=U(\mathfrak{g}) \operatorname{ker} \chi_{\Lambda_{1}}$.

Aplicando o Corolário 3.4.10, b), temos

Teorema 4.1.3 (Teorema 6.3 em [FHR20]). Todo módulo de peso máximo simples admissíveis na órbita nilpotente minimal é um módulo de $\Gamma$-relações limitado.

Observação 4.1.4. A ação de $E_{k+1, k} \mathrm{em} L(\Lambda)$ não é injetiva para nenhum $k=2,3, \ldots n-1$. De fato, considerando que $u_{1}-v_{2} \notin \mathbb{Z}$ e $v_{k-1}-v_{k} \in \mathbb{Z}_{>0}$ para todo $k=2,3, \ldots n-1$, obtemos $v_{k-2}-v_{k}-1 \in$ $\mathbb{Z}_{>0}$ para todo $k=3,4, \ldots n-1$. Isso implica que $T(w)=T\left(v+\left(v_{k}-v_{k-1}+1\right) \delta^{k k}\right) \in \mathcal{B}_{\mathcal{C}}(T(v)) \mathrm{e}$ $T\left(w-\delta^{k k}\right) \notin \mathcal{B}_{\mathcal{C}}(T(v))$ e, portanto, $E_{k+1, k}(T(w))=0$.

Seja $F:=\left\{E_{m_{i} 1} \mid i=1, \ldots, k\right\}$ de modo que $m_{i} \in\{2, \ldots, n+1\}$ para cada $i=1, \ldots, k$. Pelo Corolário 1.4.27 e Proposição 3.7.6, obtemos imediatamente

Corolário 4.1.5 (Corolário 6.5 em [FHR20]). Seja $n \geq 2$. Todos os subquocientes simples de $D_{F} L(\Lambda)$ são $\Gamma$-módulos de relações admissíveis limitados de nível $k$ na órbita minimal.

Observação 4.1.6. a) O corolário 4.1.5 para sl(3) foi provado em [AFR17, Teorema 5.6].

b) Todos os módulos simples no Corolário 4.1.5 são módulos de peso máximo (com relação a alguma subálgebra de Borel) com multiplicidades de peso limitadas. Mais especificamente, $T_{\alpha}(L(\Lambda))$ é um módulo de relações admissível simples na categoria $\mathcal{O}$, em que $\alpha=\alpha_{1, m-1}$, para cada $m \in\{2,3, \ldots, n+1\}$. 
Aplicando o 3.4.17 temos

Corolário 4.1.7 (Corolário 6.7 em [FHR20]). Seja $\left\langle\lambda+\rho, \beta_{j}^{\vee}\right\rangle \in \mathbb{Z}_{\geq 0}$ para alguma raiz simples $\beta_{j}, j=1, \ldots, t$ de $\mathfrak{b}$, e seja $w \in W$ tal que $\left\langle w(\lambda+\rho), \beta_{j}^{\vee}\right\rangle \in \mathbb{Z}_{<0}$ para cada $j=1, \ldots, t$. Então $L(\lambda) \simeq L_{w \mathfrak{b}}(w \lambda)$ é um $\Gamma_{\mathcal{F}}$-módulo de relações, em que $\Gamma_{\mathcal{F}}=w \Gamma$.

Também temos o seguinte resultado.

Corolário 4.1.8 (Corolário $6.8 \mathrm{em}$ [FHR20]). Seja $\lambda \in \mathfrak{h}^{*}, L(\lambda)=L_{\mathfrak{b}}(\lambda)$ um módulo de peso máximo admissível na órbita minimal (com relação a uma subálgebra de Borel $\mathfrak{b}$ ) e $\beta$ uma raiz simples de $\mathfrak{b}$ tal que $\left\langle\lambda, \beta^{\vee}\right\rangle \notin \mathbb{Z}_{\geq 0}$. Então

a) O módulo $L_{s_{\beta} \mathfrak{b}}\left(s_{\beta}(\lambda+\beta)\right)$ é um $s_{\beta} \Gamma$-módulo admissível de relações na órbita minimal.

b) Fixado $i>1$, seja $\beta=\beta_{i}$ a primeira raiz simples de $\mathfrak{b}$ tal que $\left\langle\lambda, \beta^{\vee}\right\rangle \notin \mathbb{Z}_{\geq 0}$. Se $w=$ $s_{i-1} s_{i} s_{i-2} s_{i-1} \cdots s_{2} s_{1} s_{3} s_{1} s_{2} \in W$, então $L_{w^{-1} \mathfrak{b}}\left(w^{-1} \lambda\right)$ é um $\Gamma$-módulo de relações na órbita minimal.

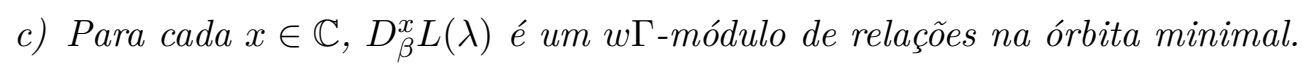

Demonstração. Seja $\Lambda=\bar{\lambda}-\frac{a p}{q} \varpi_{1}$, em que $\bar{\lambda}=\left(\lambda_{1}, \ldots, \lambda_{n}\right)$ e cada $\lambda_{i} \operatorname{com} i=1, \ldots, n$, é um inteiro não negativo. Então $\lambda=s_{t} \ldots s_{1} \cdot \Lambda$ para algum $t \leq n$. Se $t=n$ então a última coordenada de $\lambda$ é não inteira, caso em que $\beta=\alpha_{n}$. Se $t<n$ então $\lambda$ tem exatamente duas coordenadas não inteiras nas posições $t$ e $t+1$, neste caso $\beta=\alpha_{t}$ o $\beta=\alpha_{t-1}$. Consequentemente, $L\left(s_{\beta} \cdot \lambda\right)$ é um módulo de peso máximo simples admissível na órbita minimal. Mas $s_{\beta} \cdot \lambda=s_{\beta}(\lambda+\beta)$. Agora considere $L_{s_{\beta} \mathfrak{b}}\left(s_{\beta}(\lambda+\beta)\right)$. Este é o módulo de peso máximo com relação à subálgebra de Borel $s_{\beta} \mathfrak{b}$ e o correspondente peso máximo (relativo a $\left.s_{\beta} \mathfrak{b}\right)$ é $s_{\beta}(\lambda+\beta)$. Portanto, $L_{s_{\beta}} \mathfrak{b}\left(s_{\beta}(\lambda+\beta)\right.$ ) é um $\Gamma^{\prime}$-módulo de relações admissível na órbita minimal em que $\Gamma^{\prime}$ é a subálgebra Gelfand-Tsetlin padrão de $s_{\beta} \mathfrak{b}$, isto é $\Gamma^{\prime}=s_{\beta} \Gamma$. Isto mostra a afirmação do item a).

Dado que $L(\lambda)$ é um módulo na órbita nilpotente minimal, então $L_{w^{-1} \mathfrak{b}}\left(w^{-1} \lambda\right)$ também é um módulo admissível na órbita nilpotente minimal. Portanto, $L_{w^{-1} \mathfrak{b}}\left(w^{-1} \lambda\right)$ é um $w^{-1} \Gamma$-módulo de relações. A afirmação b) segue do Lema 3.4.18. Agora, torcendo $L_{w^{-1} \mathfrak{b}}\left(w^{-1} \lambda\right)$ por $w$, que está aplicando $w$ às fórmulas de Gelfand-Tsetlin, concluímos que $L(\lambda)$ é um $w \Gamma$-módulo de relações. Observe que $w \in W$ é o elemento de comprimento mínimo, de modo que $\beta$ é a primeira raiz simples de $w \mathfrak{b}$. Portanto, c) segue do Teorema 3.5.6.

\subsubsection{Classificação dos $\mathfrak{s l}_{n+1}$-módulos induzidos de $\mathfrak{s l}_{2}$-módulos densos}

Seja $k=\frac{p}{q}-n-1$ um número admissível para $\widehat{\mathfrak{g}}$. Nesta seção, concluímos a classificação de todos os g-módulos simples admissíveis na órbita minimal que são quocientes dos módulos induzidos 
a partir de subálgebras parabólicas com o fator Levi isomorfo a $\mathfrak{s l}_{2}+\mathfrak{h}$. Também mostraremos que todos esses módulos são módulos de relações e, portanto, obtemos sua realização por tabelas.

Suponhamos que $M$ seja um $\mathfrak{g}$-módulo admissível do nível $k$. Consideramos uma subálgebra parabólica $\mathfrak{p}=\mathfrak{p}_{\Sigma}=\mathfrak{l}_{\Sigma}+\mathfrak{n}_{\Sigma}$ de $\mathfrak{g}$, em que $\Sigma$ consiste em uma raiz simples $\beta$. Denotamos por $M^{\mathfrak{n}}$ o subespaço de todos os $\mathfrak{n}_{\Sigma}$-invariantes que é um $\mathfrak{n}_{\Sigma}$-módulo. Suponhamos que $M=L_{\mathfrak{p}}(\Sigma, N)$ para algum $\mathfrak{l}_{\Sigma}$-módulo de peso simples $N$ com $\mathfrak{n}_{\Sigma} N=0$. Então $M^{\mathfrak{n}} \simeq N$ é um $\mathfrak{l}_{\Sigma}$-módulo admissível de nível $k_{\beta}=\frac{2}{(\beta, \beta)}(k+n+1)-2$ por [AFR17, Teorema 2.12]. Assim temos a seguinte proposição

Proposição 4.1.9 (Proposição 6.9 em [FHR20]). Seja $\mathfrak{a}=\mathfrak{s l}_{2}, V=V(\gamma, \mu)$ um a-módulo de peso denso simples, $\gamma, \mu \in \mathbb{C}$. Então $V$ é admissível de nível $k$ na órbita minimal se, e somente se, $\mu=\lambda-\frac{a p}{q}+2 x$ e $\gamma=\left(\lambda-\frac{a p}{q}+1\right)^{2}$, em que $\lambda \in\{0,1, \ldots, p-2\}, a \in\{1, \ldots, q-1\}, x \in \mathbb{C} \backslash \mathbb{Z}$ e $x-\frac{a p}{q} \notin \mathbb{Z}$.

Demonstração. Segue da Proposição 3.5.8.

Observação 4.1.10. Na proposição acima temos $V=V_{\mathcal{C}}(T(v))$, em que $\mathcal{C}=\emptyset, T(v)=T\left(v_{i j}\right)$ é a tabela Gelfand-Tsetlin com altura 2, de modo que $v_{11}=\frac{\mu}{2}, v_{21}=\frac{1}{2}\left(\lambda-\frac{a p}{q}\right)$ e $v_{22}=\frac{1}{2}\left(\frac{a p}{q}-\lambda-2\right)$ (cf. Teorema 3.5.9). O conjunto $\mathcal{B}_{\mathcal{C}}(T(v))=\left\{T\left(v+\ell \delta^{11}\right) \mid \ell \in \mathbb{Z}\right\}$ é uma base de $V$.

Corolário 4.1.11 (Corolário 6.11 em [FHR20]). Seja $n>1, \gamma \in \mathbb{C}, \mu=\left(\mu_{1}, \ldots, \mu_{n}\right) \in \mathfrak{h}^{*}$, de modo que $V \simeq V\left(\gamma, \mu_{1}\right)$ é um $\mathfrak{s l}_{2}$-módulo de peso denso simples e $\mu_{i}=\left\langle\mu, \alpha_{i}^{\vee}\right\rangle, i=1, \ldots, n$. Seja $L(\gamma, \mu)=L_{\mathfrak{p}}^{\mathfrak{g}}(\{\alpha\}, V)$. Então $L(\gamma, \mu)$ é admissível de nível $k$ na órbita minimal se, e somente se, $\mu_{1}=\lambda_{1}-\frac{a p}{q}+2 x, \mu_{2}=\lambda_{2}-x, \mu_{j}=\lambda_{j}$ para todo $j=3, \ldots, n$ e $\gamma=\left(\lambda_{1}-\frac{a p}{q}+1\right)^{2}$ com $\left\{\lambda_{i}\right\}_{i=1, \ldots, n} \subset \mathbb{Z}_{\geq 0}$, de modo que $\lambda_{1}+\ldots+\lambda_{n}<p-n, a \in\{1,2, \ldots, q-1\}, x \in \mathbb{C} \backslash \mathbb{Z}$ e $x-\frac{a p}{q} \notin \mathbb{Z}$.

Demonstração. Segue da Proposição 4.1.9, Teorema 2.4.5 e Teorema 3.5.9.

Teorema 4.1.12 (Teorema 6.12 em [FHR20]). Seja $\beta$ uma raiz de $\mathfrak{g}, \mathfrak{b}$ uma subálgebra de Borel de $\mathfrak{g}$ para qual $\beta$ é uma raiz positiva, $\rho_{\beta}$ um meio da soma das raízes positivas (com relação a $\mathfrak{b})$. Seja $L_{\mathfrak{b}}(\lambda)$ um $\mathfrak{b}$-módulo de peso máximo simples admissível na órbita minimal, de modo que $\left\langle\lambda+\rho_{\beta}, \beta^{\vee}\right\rangle \notin \mathbb{Z}$ e $f=f_{\beta}$. Denotamos por $A_{\mathfrak{b}, \beta}$ o conjunto de todos os $x \in \mathbb{C} \backslash \mathbb{Z}$ de forma que $x+\left\langle\lambda+\rho_{\beta}, \beta^{\vee}\right\rangle \notin \mathbb{Z}$.

a) $O \mathfrak{g}$-módulo $D_{f_{\beta}}^{x} L_{\mathfrak{b}}(\lambda)$ é admissível na órbita minimal para qualquer $x \in A_{\mathfrak{b}, \beta}$;

b) Os módulos $D_{f_{\beta}}^{x} L_{\mathfrak{b}}(\lambda)$, em que $\mathfrak{g}_{\beta} \subset \mathfrak{b}, x \in A_{\mathfrak{b}, \beta},\left\langle\lambda+\rho_{\beta}, \beta^{\vee}\right\rangle \notin \mathbb{Z}$ e $L_{\mathfrak{b}}(\lambda)$ é admissivel na órbita minimal, esgotam todos os módulos simples admissíveis induzidos por $\mathfrak{s l}_{2}$ na órbita minimal. Todos esses módulos têm multiplicidades de peso limitadas;

c) Existe uma bandeira $\mathcal{F}$ tal que $D_{f}^{x} L_{\mathfrak{b}}(\lambda)$ é um $\mathfrak{g}$-módulo de $\Gamma_{\mathcal{F}}$-relações.

Demonstração. Asumiremos que $\mathfrak{b}$ é a subálgebra padrão do Borel de $\mathfrak{g}$. Para uma subálgebra de Borel qualquer o argumento é análogo. Primeiro, provamos a). Seja $\beta=\alpha_{r}+\ldots+\alpha_{t}$ para algumas raízes simples consecutivas $\alpha_{j}, j=r, \ldots, t$. Uma vez que $\lambda$ é admissível na órbita minimal, então temos duas possibilidades: ou existe apenas uma raiz simples $i$ tal que $\left\langle\lambda, \alpha_{i}^{\vee}\right\rangle \notin \mathbb{Z}$ ou existem apenas duas dessas raízes que são consecutivas. Consideremos o primeiro caso. Então ou $i=r=1$ 
ou $i=t=n$. Sem perda de generalidade, podemos assumir que $i=1$ (se $i=n$ podemos aplicar a simetria do sistema de raízes) e, portanto, $\left\langle\lambda+\rho, \alpha_{j}^{v}\right\rangle \in \mathbb{Z}_{>0}, j=2, \ldots, t$. Escolhamos a seguinte base do sistema de raízes

$$
\pi^{\prime}=\left\{\beta,-\alpha_{t}, \ldots,-\alpha_{2}, \alpha_{2}+\ldots+\alpha_{t+1}, \alpha_{t+2}, \ldots, \alpha_{n}\right\}
$$

Seja $w \in W$ tal que $w \pi=\pi^{\prime}$. Então $w$ satisfaz o Corolário 4.1 .7 e $L(\lambda) \simeq L_{w \mathfrak{b}_{s t}}(w \lambda)$ é um $w \Gamma$-módulo de relações. Então a afirmação segue do Teorema 3.5.6.

Agora consideremos o segundo caso. Suponhamos que $\left\langle\lambda, \alpha_{i}^{\vee}\right\rangle \notin \mathbb{Z}, i=k, k+1$ para algum $k$. Se $\beta=\alpha_{k}$ então a afirmação segue do Corolário 4.1.8, item c). Se $\beta=\alpha_{r}+\ldots+\alpha_{k}$ então aplicamos a simetria do diagrama Dynkin e Corolário 4.1.8, item c). Seja $\beta=\alpha_{r}+\ldots+\alpha_{k}$ para algum $1 \leq r \leq k-1$. Escolhamos $w \in W$ de modo que $w \pi$ contenha $\beta$ e $-\alpha_{r}, \ldots,-\alpha_{k-1}$ (tal $w$ existe claramente). Então $L(\lambda) \simeq L_{w \mathfrak{b}_{s t}}(w \lambda)$. Consequentemente, o problema se reduz ao caso $\beta=\alpha_{k}$ que foi discutido antes. Se $\beta=\alpha_{k+1}+\ldots+\alpha_{t}$ para algum $k+1 \leq t \leq n$, então a declaração segue da simetria do diagrama Dynkin. Finalmente b) e c) seguem de a) e do Teorema 3.5.9.

Observamos que o Teorema 4.1.12 foi inicialmente comprovado para $\mathfrak{g}=\mathfrak{s l}(3)$ in $[$ AFR17, Teorema 5.6].

Seja $\beta$ uma raiz de $\mathfrak{g}$ e seja $\mathfrak{b}$ uma subálgebra de Borel de $\mathfrak{g}$ para a qual $\beta$ é a primeira raiz simples (tal $\mathfrak{b}$ sempre existe seja pela conjugação pelo grupo de Weyl ou pela simetria do diagrama de Dynkin). Seja $\mathfrak{p}=\mathfrak{a}_{\beta} \oplus \mathfrak{n}$ uma subálgebra parabólica de $\mathfrak{g}$ contendo $\mathfrak{b}$ com o fator Levi $\mathfrak{a}_{\beta} \simeq \mathfrak{s l}_{2}+\mathfrak{h}$ com base na raiz $\beta$. Seja $V=V\left(\gamma_{\beta}, \mu\right), \gamma_{\beta} \in \mathbb{C}, \mu=\mu_{1} \varpi_{1}+\ldots+\mu_{n} \varpi_{n} \in \mathfrak{h}^{*}$, em que $\gamma_{\beta}$ é o autovalor do elemento Casimir de $\mathfrak{a}_{\beta},\left\langle\varpi_{i}, \beta_{j}^{\vee}\right\rangle=\delta_{i j}, i=1, \ldots, n$.

Corolário 4.1.13 (Corolário 6.12 em [FHR20]). O módulo $L_{\mathfrak{b}, \beta}\left(\gamma_{\beta}, \mu\right):=L_{\mathfrak{p}}^{\mathfrak{g}}(V)$ é admissível na órbita minimal se, e somente se, $\left\langle\mu-\lambda, \beta_{1}^{\vee}\right\rangle=2 x,\left\langle\mu-\lambda, \beta_{2}^{\vee}\right\rangle=-x,\left\langle\mu-\lambda, \beta_{i}^{\vee}\right\rangle=0$ para cada $i=3, \ldots, n$ e $\gamma_{\beta}=\left\langle\lambda+\rho_{\mathfrak{b}}, \beta_{1}^{\vee}\right\rangle^{2} \operatorname{com}\left\langle\lambda+\frac{a p}{q} \varpi_{1}+\rho_{\mathfrak{b}}, \beta_{1}^{\vee}\right\rangle \in \mathbb{Z}_{>0},\left\langle\lambda+\rho_{\mathfrak{b}}, \beta_{i}^{\vee}\right\rangle \in \mathbb{Z}_{>0}$ para todo $i \in\{2, \ldots, n\},\left\langle\lambda+\frac{a p}{q} \varpi_{1}+\rho_{\mathfrak{b}}, \beta_{1, n}^{\vee}\right\rangle<p, a \in\{1,2, \ldots, q-1\}, x \in \mathbb{C} \backslash \mathbb{Z} e x+\left\langle\lambda+\rho_{\mathfrak{b}}, \beta_{1}^{\vee}\right\rangle \notin \mathbb{Z}$.

Observação 4.1.14. O Teorema 4.1.12 fornece um algoritmo de como listar todos os módulos admissíveis simples induzidos por $\mathfrak{s l}_{2}$ na órbita minimal:

a) Considerando todas as subálgebras de Borel possíveis de $\mathfrak{g}$ contendo $\mathfrak{h}$;

b) Para cada subálgebra de Borel $\mathfrak{b}$, descrevemos $\lambda \in \mathfrak{h}^{*}$ para o qual $L_{\mathfrak{b}}(\lambda)$ é admissível usando a classificação de Arakawa para a Borel padrão e aplicando o grupo de Weyl;

c) Escolhamos qualquer raiz positiva (em relação a $\mathfrak{b}$ ) $\beta$ tal que $\left\langle\lambda+\rho, \beta^{\vee}\right\rangle \notin \mathbb{Z}$;

d) Definamos $D_{f}^{x} L_{\mathfrak{b}}(\lambda)$ para qualquer $x \in \mathbb{C} \backslash \mathbb{Z}$ de forma que $x+\left\langle\lambda+\rho, \beta^{\vee}\right\rangle \notin \mathbb{Z}$, em que $f=f_{\beta}$.

Os módulos obtidos esgotam todos os módulos admissíveis simples induzidos por $\mathfrak{s l}_{2}$ na órbita minimal. Desse modo, a prova do Teorema 4.1.12 explica como definir a bandeira $\mathcal{F}$ para a qual $L_{\mathfrak{b}, \beta}(N)$ é um $\mathfrak{g}$-módulo de $\Gamma_{\mathcal{F}}$-relações e, portanto, nos permite realizar explicitamente por tabelas todos esses módulos.

No próximo exemplo aplicamos o algoritmo anterior. 
Exemplo 4.1.15. Seja $\lambda_{1}=\lambda_{2}=0, p=n=3, q=2, a=1$ e $L\left(\Lambda_{i}\right)$ para $i=1,2,3$ como no Exemplo 4.1.1. Consideremos o caso $\lambda=\Lambda_{1}$ (os outros dois casos são similares). Como $L(\lambda) \simeq L_{\mathfrak{b}}(\mu)$ em que $\mu=s_{2} \lambda=\lambda$ e $\mathfrak{b}=s_{2} \mathfrak{b}_{s t}=\left\langle E_{12}, E_{32}, E_{13}, h_{1}+h_{2},-h_{2}\right\rangle$. Além da Borel padrão, temos a Borel b. Com efeito, os únicos módulos induzidos de $\mathfrak{s l}_{2}$-módulos densos na órbita minimal são:

- $D_{f}^{x} L(\lambda)$ para qualquer $x \in \mathbb{C} \backslash \mathbb{Z}$ de forma que $x-\frac{1}{2} \notin \mathbb{Z}$, em que $f=f_{\alpha_{1}}$;

- $D_{f}^{x} L_{\mathfrak{b}}(\mu)$ para qualquer $x \in \mathbb{C} \backslash \mathbb{Z}$ de forma que $x+\frac{1}{2} \notin \mathbb{Z}$, em que $f=f_{\alpha_{1}+\alpha_{2}}$.

Lembramos que para aplicar o funtor de localização torcida ao módulo $L_{\mathfrak{b}}(\mu)$ com relação a $f_{\alpha_{1}+\alpha_{2}}$, precisamos mudar a base do sistema de raízes para $\pi^{\prime}=s_{2} \pi=\left\{\alpha_{1}+\alpha_{2},-\alpha_{2}\right\}$, na qual $\alpha_{1}+\alpha_{2}$ é a primeira raiz simples positiva.

\section{$4.2 \mathfrak{s l}_{3}$-módulos de $\Gamma_{\mathcal{F}}$-relações na órbita minimal}

Nesta seção vamos usar o funtor de localização torcida como ferramenta para provar que todos os $\mathfrak{s l}_{3}$-módulos de Gelfand-Tsetlin na órbita minimal são módulos de relações. Aproveitando que temos uma descrição explícita das bases para cada módulo simples de relações, realizaremos $\mathfrak{s l}_{3}$-módulos densos admissíveis, os quais serão obtidos a partir de módulos de peso máximo usando localização torcida com relação a $f_{\alpha}=E_{21}$ e $f_{\beta}=E_{31}$. Aqui fixamos $\Gamma=\Gamma_{s t}$ e $L(\Lambda)=L\left(\lambda_{1}-\frac{a p}{q}, \lambda_{2}\right)$ o módulo de peso máximo na órbita minimal. Também definimos $v_{1}-v_{2}=\left\langle\Lambda+\rho, \alpha_{1}^{\vee}\right\rangle \notin \mathbb{Z}$, $v_{2}-v_{3}=\left\langle\Lambda+\rho, \alpha_{2}^{\vee}\right\rangle \in \mathbb{Z}_{>0}$ e $v_{1}+v_{2}+v_{3}=-3$, pela Proposição 3.8.1 temos que o módulo $L(\Lambda)$ pode ser realizado como um módulo de relações $V_{\mathcal{C}}(T(v))$ em que $T(v)$ é a tabela genérica

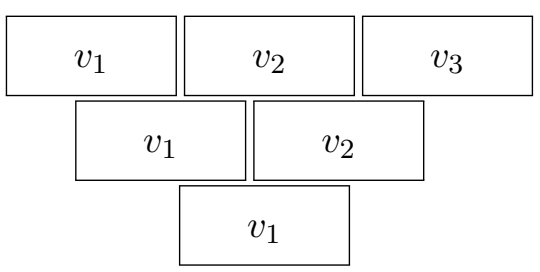

e $\mathcal{C}$ é o conjunto das relações definidas pelo seguinte grafo:

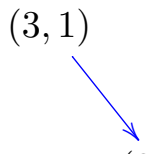

$(2,1)$
$(3,2)$ 
Uma base para este módulo é dada pelo conjunto de tabelas

$$
\mathcal{B}_{\mathcal{C}}(T(v))=L\left(\left\{\begin{array}{c}
l \leq m \leq 0 \\
-\lambda_{2}<n \leq 0
\end{array}\right) .\right.
$$

\subsubsection{Localização torcida em relação ao conjunto $\left\{f_{\alpha}, f_{\beta}\right\}$}

Antes de enunciar o próximo teorema, lembremos que:

$$
\begin{array}{llll}
{\left[E_{31}, E_{11}\right]=E_{31}} & {\left[E_{31}, E_{12}\right]=E_{32}} & {\left[E_{31}, E_{13}\right]=-\left(H_{1}+H_{2}\right)} \\
{\left[E_{31}, E_{21}\right]=0} & {\left[E_{31}, E_{22}\right]=0} & {\left[E_{31}, E_{23}\right]=-E_{21}} \\
{\left[E_{31}, E_{31}\right]=0} & {\left[E_{31}, E_{32}\right]=0} & {\left[E_{31}, E_{33}\right]=-E_{31}}
\end{array}
$$

Consequentemente,

$$
\begin{array}{lll}
a d^{2}\left(E_{31}\right)\left(E_{11}\right)=0 & a d^{2}\left(E_{31}\right)\left(E_{12}\right)=0 & a d^{3}\left(E_{31}\right)\left(E_{13}\right)=0 \\
\operatorname{ad}\left(E_{31}\right)\left(E_{21}\right)=0 & a d\left(E_{31}\right)\left(E_{22}\right)=0 & a d^{2}\left(E_{31}\right)\left(E_{23}\right)=0 \\
\operatorname{ad}\left(E_{31}\right)\left(E_{31}\right)=0 & a d\left(E_{31}\right)\left(E_{32}\right)=0 & a d^{2}\left(E_{31}\right)\left(E_{33}\right)=0
\end{array}
$$

Lema 4.2.1. A ação torcida dos geradores de $\mathfrak{s l}_{3}$ em relação ao conjunto $\left\{f_{\alpha}, f_{\beta}\right\}$ será dada por:

- $\Theta_{\left\{f_{\alpha}, f_{\beta}\right\}}^{(x, y)}\left(E_{21}\right)=E_{21}$

- $\Theta_{\left\{f_{\alpha}, f_{\beta}\right\}}^{(x, y)}\left(E_{12}\right)=E_{12}-x\left(H_{1}+x+y-1\right) E_{21}^{-1}+y E_{32} E_{31}^{-1}$

- $\Theta_{\left\{f_{\alpha}, f_{\beta}\right\}}^{(x, y)}\left(E_{32}\right)=E_{32}-x E_{31} E_{21}^{-1}$

- $\Theta_{\left\{f_{\alpha}, f_{\beta}\right\}}^{(x, y)}\left(E_{23}\right)=E_{23}-y E_{21} E_{31}^{-1}$

- $\Theta_{\left\{f_{\alpha}, f_{\beta}\right\}}^{(x, y)}\left(E_{31}\right)=E_{31}$

- $\Theta_{\left\{f_{\alpha}, f_{\beta}\right\}}^{(x, y)}\left(E_{13}\right)=E_{13}+x E_{23} E_{21}^{-1}-y\left(H_{1}+H_{2}+x+y-1\right) E_{31}^{-1}$

- $\Theta_{\left\{f_{\alpha}, f_{\beta}\right\}}^{(x, y)}\left(H_{1}\right)=H_{1}+2 x+y$

- $\Theta_{\left\{f_{\alpha}, f_{\beta}\right\}}^{(x, y)}\left(H_{2}\right)=H_{2}-x+y$

Demonstração. A ação dos geradores de $\mathfrak{s l}_{3}$ no módulo localizado torcido em relação a $E_{31}$ será dada por:

- $\Theta_{f_{\beta}}^{y}\left(E_{12}\right)=E_{12}+y E_{32} E_{31}^{-1}$

- $\Theta_{f_{\beta}}^{y}\left(E_{13}\right)=E_{13}-y\left(H_{1}+H_{2}+y-1\right) E_{31}^{-1}$ 
- $\Theta_{f_{\beta}}^{y}\left(E_{21}\right)=E_{21}$

- $\Theta_{f_{\beta}}^{y}\left(E_{23}\right)=E_{23}-y E_{21} E_{31}^{-1}$

- $\Theta_{f_{\beta}}^{y}\left(E_{31}\right)=E_{31}$

- $\Theta_{f_{\beta}}^{y}\left(E_{32}\right)=E_{32}$

- $\Theta_{f_{\beta}}^{y}\left(H_{1}\right)=H_{1}+y$

- $\Theta_{f_{\beta}}^{y}\left(H_{2}\right)=H_{2}+y$

Lema 4.2.2. Sejam $\left\{c_{i j}\right\}_{1 \leq j \leq i \leq 3}$ os geradores de $\Gamma$ definidos em 1. Então

$$
\Theta_{\left\{f_{\alpha}, f_{\beta}\right\}}^{(x, y)}\left(c_{i j}\right)= \begin{cases}c_{11}+x+y, & \text { se }(i, j)=(1,1) \\ c_{21}+y, & \text { se }(i, j)=(2,1) \\ c_{22}+y\left(2 E_{11}+y-1\right)+2 y E_{32} E_{21} E_{31}^{-1}, & \text { se }(i, j)=(2,2) \\ c_{i j}, & \text { se } i=3 \text { e } j=1,2,3 .\end{cases}
$$

Demonstração. Como $c_{11}=E_{11}$ e $c_{21}=E_{11}+E_{22}$, temos $\operatorname{ad}\left(E_{31}\right)\left(c_{11}\right)=\operatorname{ad}\left(E_{31}\right)\left(c_{21}\right)=E_{31} \mathrm{e}$ $a d^{2}\left(E_{31}\right)\left(c_{11}\right)=a d^{2}\left(E_{31}\right)\left(c_{21}\right)=0$. Ademais, $c_{22}=E_{11} E_{11}+E_{12} E_{21}+E_{21} E_{12}+E_{22} E_{22}, \log$, $a d\left(E_{31}\right)\left(c_{22}\right)=2\left(E_{11} E_{31}+E_{32} E_{21}\right), a d^{2}\left(E_{31}\right)\left(c_{22}\right)=2 E_{31}^{2}$ e $a d^{3}\left(E_{31}\right)\left(c_{22}\right)=0$. Consequentemente,

$$
\Theta_{\left\{f_{\alpha}, f_{\beta}\right\}}^{(x, y)}\left(c_{11}\right)=\Theta_{\left\{f_{\alpha}, f_{\beta}\right\}}^{(x, y)}\left(E_{11}\right)=\sum_{i, j \geq 0}\left(\begin{array}{c}
x \\
i
\end{array}\right)\left(\begin{array}{c}
y \\
j
\end{array}\right) \operatorname{ad}\left(f_{\alpha}\right)^{i} \operatorname{ad}\left(f_{\beta}\right)^{j}\left(E_{11}\right) f_{\alpha}^{-i} f_{\beta}^{-j}=E_{11}+x+y .
$$

Por outro lado, sabemos que $c_{21}$ e $c_{22}$ comutam com $E_{21}$, logo

$$
\Theta_{\left\{f_{\alpha}, f_{\beta}\right\}}^{(x, y)}\left(c_{21}\right)=\Theta_{f_{\beta}}^{y}\left(c_{21}\right)=\sum_{j \geq 0}\left(\begin{array}{c}
y \\
j
\end{array}\right) \operatorname{ad}\left(f_{\beta}\right)^{j}\left(c_{21}\right) f_{\beta}^{-j}=c_{21}+y E_{31} E_{31}^{-1}
$$

$\mathrm{e}$

$$
\Theta_{\left\{f_{\alpha}, f_{\beta}\right\}}^{(x, y)}\left(c_{22}\right)=\Theta_{f_{\beta}}^{y}\left(c_{22}\right)=c_{22}+y\left(2\left(E_{11} E_{31}+E_{32} E_{21}\right)\right) E_{31}^{-1}+\frac{y(y+1)}{2}\left(2 E_{31}^{2}\right) E_{31}^{-1}
$$

Finalmente, desde que $c_{3 j}$ comuta com $E_{21}$ e $E_{31}$ para cada $j=1,2,3$, temos $\Theta_{\left\{f_{\alpha}, f_{\beta}\right\}}^{(x, y)}\left(c_{3 j}\right)=$ $c_{3 j}$

Proposição 4.2.3. A localização de $L(\Lambda)$ com relação ao conjunto $\left\{f_{\alpha}, f_{\beta}\right\}$ torcida pelo automorfismo $\Theta_{\left\{f_{\alpha}, f_{\beta}\right\}}^{(x, y)}$ é o módulo Gelfand-Tsetlin $V_{\mathcal{D}}(T(v))$ em que $\mathcal{D}$ é o conjunto das relações definido pelo grafo seguinte: 
com base determinada pelo conjunto de tabelas:

$$
\mathcal{B}_{\mathcal{D}}(T(v))=L\left(\left\{\begin{array}{c}
-\lambda_{2} \leq n \leq 0 \\
l, m \in \mathbb{Z}
\end{array}\right)\right.
$$

e a ação de $\mathfrak{s l}_{3}$ em $V_{\mathcal{D}}(T(v))$ e a ação torcida dos geradores de $\mathfrak{s l}_{3}$ no módulo localizado será dada por:

- $H_{1} \cdot T(w)^{(x, y)}=\left(\lambda_{w}^{1}+2 x+y\right) T(w)^{(x, y)}$

- $H_{2} \cdot T(w)^{(x, y)}=\left(\lambda_{w}^{2}+y-x\right) T(w)^{(x, y)}$

- $E_{21} \cdot T(w)^{(x, y)}=T\left(w-\delta^{11}\right)^{(x, y)}$

- $E_{32} \cdot T(w)^{(x, y)}=\frac{w_{21}-\left(w_{11}+x\right)}{w_{21}-w_{22}} T\left(w-\delta^{21}\right)^{(x, y)}+\frac{w_{22}-\left(w_{11}+x\right)}{w_{22}-w_{21}} T\left(w-\delta^{22}\right)^{(x, y)}$

$$
\begin{aligned}
E_{12} \cdot T(w)^{(x, y)} & =-\left(w_{11}+x+y-w_{21}\right)\left(w_{11}+x+y-w_{22}\right) T\left(w+\delta^{11}\right)^{(x, y)} \\
& +y \sum_{i=2}^{d}\left(w_{21}-w_{11}+i\right) T\left(w+\delta^{11}+(i-1) \delta^{21}-(i-1) \delta^{22}\right)^{(x, y)} \\
& +y \sum_{i=1}^{d-1}\left(w_{22}-w_{11}-i+1\right) T\left(w+\delta^{11}+i \delta^{21}-i \delta^{22}\right)^{(x, y)}
\end{aligned}
$$

$$
\begin{aligned}
E_{23} \cdot T(w)^{(x, y)} & =\frac{\left(w_{21}-w_{31}\right)\left(w_{21}-w_{32}\right)\left(w_{21}-w_{33}\right)}{\left.w_{22}-w_{21}\right)} T\left(w+\delta^{21}\right)^{(x, y)} \\
& +\frac{\left(w_{22}-w_{31}\right)\left(w_{22}-w_{32}\right)\left(w_{22}-w_{33}\right)}{w_{21}-w_{22}} T\left(w+\delta^{22}\right)^{(x, y)} \\
& -y \sum_{i=1}^{d}\left(w_{21}-w_{22}+2 i-1\right) T\left(w+i \delta^{21}-(i-1) \delta^{22}\right)^{(x, y)}
\end{aligned}
$$

em que $d:=\lambda_{2}+1$.

Demonstração. É preciso lembrar que, para cada $T(w) \in \mathcal{B}_{\mathcal{C}}(T(v))$, temos $E_{21}(T(w))=T(w-$ $\left.\delta^{11}\right) \in \mathcal{B}_{\mathcal{C}}(T(v))$, uma vez que $((1,1) ;(2,1)) \notin \mathcal{C}$. Logo, $E_{21}^{-1}(T(w))=T\left(w+\delta^{11}\right)$, para cada $T(w) \in \mathcal{B}_{\mathcal{C}}(T(v))$

Para determinar $E_{31}^{-1}(T(w))$, podemos proceder de forma análoga. Isto é, suponhamos que $T(w) \in \mathcal{B}_{\mathcal{C}}(T(v))$, então $T\left(w+\delta^{11}+\delta^{21}\right) \in \mathcal{B}_{\mathcal{C}}(T(v))$, pois $((3,1) ;(2,1)) \notin \mathcal{C}$. Logo, podemos usar a seguinte identidade formal ${ }^{1}$ :

$$
E_{31}\left(T\left(w+\delta^{11}+\delta^{21}\right)\right)=\frac{1}{\left(w_{21}+1\right)-w_{22}} T(w)+\frac{1}{w_{22}-\left(w_{21}+1\right)} T\left(w+\delta^{21}-\delta^{22}\right)
$$

Ademais, se $T(w)$ é um tabela em $\mathcal{B}_{\mathcal{C}}(T(v))$ então $w_{22}-w_{33} \in\{1,2, \ldots, d\}$. Portanto, temos um número finito de casos.

1. Caso: $w_{22}-w_{33}=1$.

Neste caso $T\left(w+\delta^{21}-\delta^{22}\right) \notin \mathcal{B}_{\mathcal{C}}(T(v))$, uma vez que $\left(w_{22}-1\right)-w_{33}=w_{22}-w_{33}-1=0$. $\mathrm{O}$ que implica que:

$$
E_{31}\left(T\left(w+\delta^{11}+\delta^{21}\right)\right)=\frac{1}{\left(w_{21}+1\right)-w_{22}} T(w)
$$

\footnotetext{
${ }^{1} \mathrm{O}$ coeficiente correspondente à tabela que não satisfaz $\mathcal{C}$ é nulo por definição.
} 
Portanto,

$$
T(w)=E_{31}\left(\left(w_{21}-w_{22}+1\right) T\left(w+\delta^{11}+\delta^{21}\right)\right)
$$

2. Caso: $w_{22}-w_{33}=2$.

Neste caso $T\left(w+\delta^{21}-\delta^{22}\right) \in \mathcal{B}_{\mathcal{C}}(T(v))$, já que $w_{32}-\left(w_{22}-1\right)=w_{32}-w_{22}+1 \in \mathbb{Z}_{>0}$, $\left(w_{22}-1\right)-w_{33}=w_{22}-w_{33}-1=1,((1,1) ;(2,1)) \notin \mathcal{C}$ e $((3,1) ;(2,1)) \notin \mathcal{C}$. Por conseguinte, podemos aplicar o caso anterior e temos:

$$
\begin{aligned}
T\left(w+\delta^{21}-\delta^{22}\right) & =E_{31}\left(\left(w_{21}+1-\left(w_{22}-1\right)+1\right) T\left(w+\delta^{21}-\delta^{22}+\delta^{11}+\delta^{21}\right)\right) \\
& =E_{31}\left(\left(w_{21}-w_{22}+3\right) T\left(w+\delta^{11}+2 \delta^{21}-\delta^{22}\right)\right)
\end{aligned}
$$

Assim,

$$
\begin{aligned}
E_{31}\left(T\left(w+\delta^{11}+\delta^{21}\right)\right) & =\frac{1}{\left(w_{21}+1\right)-w_{22}} T(w)+\frac{1}{w_{22}-\left(w_{21}+1\right)} T\left(w+\delta^{21}-\delta^{22}\right) \\
& =\frac{1}{w_{21}-w_{22}+1} T(w)-E_{31}\left(\frac{w_{21}-w_{22}+3}{w_{21}-w_{22}+1} T\left(w+\delta^{11}+2 \delta^{21}-\delta^{22}\right)\right)
\end{aligned}
$$

Resolvendo para $T(w)$, temos: $T(w)=E_{31}\left(T\left(w^{\prime}\right)\right)$. Em que,

$T\left(w^{\prime}\right)=\left(w_{21}-w_{22}+1\right) T\left(w+\delta^{11}+\delta^{21}\right)+\left(w_{21}-w_{22}+3\right) T\left(w+\delta^{11}+2 \delta^{21}-\delta^{22}\right) \in V_{\mathcal{C}}(T(v))$.

3. Caso: $w_{22}-w_{33}=i \in\{3,4, \ldots, d\}$.

Neste caso $T\left(w+\delta^{21}-\delta^{22}\right) \in \mathcal{B}_{\mathcal{C}}(T(v))$, uma vez que $w_{32}-\left(w_{22}-1\right)=w_{32}-w_{22}+1 \in \mathbb{Z}_{>0}$, $\left(w_{22}-1\right)-w_{33}=w_{22}-w_{33}-1=i-1 \in\{2,3, \ldots, d-1\},((1,1) ;(2,1)) \notin \mathcal{C}$ e $((3,1) ;(2,1)) \notin \mathcal{C}$. Portanto, aplicando o caso anterior, provamos a existência de $T\left(w_{0}\right) \in V_{\mathcal{C}}(T(v))$ tal que $T\left(w+\delta^{21}-\delta^{22}\right)=E_{31}\left(T\left(w_{0}\right)\right)$.

Consequentemente,

$$
\begin{aligned}
E_{31}\left(T\left(w+\delta^{11}+\delta^{21}\right)\right) & =\frac{1}{\left(w_{21}+1\right)-w_{22}} T(w)+\frac{1}{w_{22}-\left(w_{21}+1\right)} T\left(w+\delta^{21}-\delta^{22}\right) \\
& =\frac{1}{w_{21}-w_{22}+1} T(w)-E_{31}\left(\frac{1}{w_{21}-w_{22}+1} T\left(w_{0}\right)\right)
\end{aligned}
$$

Logo, $T(w)=E_{31}\left(T\left(w^{\prime}\right)\right)$. Em que,

$$
T\left(w^{\prime}\right)=\left(w_{21}-w_{22}+1\right) T\left(w+\delta^{11}+\delta^{21}\right)+T\left(w_{0}\right) \in V_{\mathcal{C}}(T(v)) .
$$

Dos casos 1, 2 e 3, concluímos que para cada $T(w) \in \mathcal{B}_{\mathcal{C}}(T(v))$, existe $T\left(w^{\prime}\right) \in V_{\mathcal{C}}(T(v))$ tal que $T(w)=E_{31}\left(T\left(w^{\prime}\right)\right)$. Portanto, para cada $u \in V_{\mathcal{C}}(T(L))$, temos

$$
u=\sum_{i} a_{i \in I} T\left(w_{i}\right)=\sum_{i \in I} a_{i} E_{31}\left(T\left(w_{i}^{\prime}\right)\right)=E_{31}\left(\sum_{i \in I} a_{i} T\left(w_{i}^{\prime}\right)\right) .
$$


Consequentemente, temos que se $T(w) \in \mathcal{B}_{\mathcal{C}}(T(v))$, então $T\left(w+\delta^{11}+\delta^{21}\right) \in \mathcal{B}_{\mathcal{C}}(T(v))$ e

$$
T(w)=E_{31}\left(\left(w_{21}-w_{22}+1\right) T\left(w+\delta^{11}+\delta^{21}\right)\right)+T\left(w+\delta^{21}-\delta^{22}\right)
$$

portanto,

$$
T(w)=E_{31}\left(\sum_{i=1}^{d}\left(w_{21}-w_{22}+2 i-1\right) T\left(w+\delta^{11}+i \delta^{21}-(i-1) \delta^{22}\right)\right) .
$$

em que $d=\lambda_{2}+1$.

Assim,

- $H_{1} \cdot T(w)^{(x, y)}=\Theta_{\left\{f_{\alpha}, f_{\beta}\right\}}^{(x, y)}\left(H_{1}\right)(T(w))=H_{1}(T(w))+(2 x+y) T(w)=\left(\lambda_{w}^{1}+2 x+y\right) T(w)$

- $H_{2} \cdot T(w)^{(x, y)}=\Theta_{\left\{f_{\alpha}, f_{\beta}\right\}}^{(x, y)}\left(H_{2}\right)(T(w))=H_{2}(T(w))+(y-x) T(w)=\left(\lambda_{w}^{2}+y-x\right) T(w)$

- $E_{21} \cdot T(w)^{(x, y)}=\Theta_{\left\{f_{\alpha}, f_{\beta}\right\}}^{(x, y)}\left(E_{21}\right)(T(w))=E_{21}(T(w))=T\left(w-\delta^{11}\right)$

- $=\frac{1}{w_{21}-w_{22}} T\left(w-\delta^{11}-\delta^{21}\right)+\frac{1}{w_{22}-w_{21}} T\left(w-\delta^{11}-\delta^{22}\right)$

$E_{32} \cdot T(w)^{(x, y)}=\Theta_{\left\{f_{\alpha}, f_{\beta}\right\}}^{(x, y)}\left(E_{32}\right)(T(w))=\Theta_{f_{\alpha}}^{x}\left(E_{32}\right)(T(w))$

$=\frac{w_{21}-\left(w_{11}+x\right)}{w_{21}-w_{22}} T\left(w-\delta^{21}\right)+\frac{w_{22}-\left(w_{11}+x\right)}{w_{22}-w_{21}} T\left(w-\delta^{22}\right)$

$E_{12} \cdot T(w)^{(x, y)}=\Theta_{\left\{f_{\alpha}, f_{\beta}\right\}}^{(x, y)}\left(E_{12}\right)(T(w))=E_{12}(T(w))-x\left(H_{1}+x+y-1\right) E_{21}^{-1}(T(w))$

$+y E_{32} E_{31}^{-1}(T(w))$

$=-\left(w_{11}-w_{21}\right)\left(w_{11}-w_{22}\right) T\left(w+\delta^{11}\right)-x\left(\lambda_{w}^{1}+x+y+1\right)\left(T\left(w+\delta^{11}\right)\right)$

$+y \sum_{i=1}^{d}\left(w_{21}-w_{22}+2 i-1\right) E_{32}\left(T\left(w+\delta^{11}+i \delta^{21}-(i-1) \delta^{22}\right)\right)$

$=-\left(w_{11}+x+y-w_{21}\right)\left(w_{11}+x+y-w_{22}\right) T\left(w+\delta^{11}\right)$

$+y \sum_{i=2}^{d}\left(w_{21}-w_{11}+i\right) T\left(w+\delta^{11}+(i-1) \delta^{21}-(i-1) \delta^{22}\right)$

$+\quad y \sum_{i=1}^{d-1}\left(w_{22}-w_{11}-i+1\right) T\left(w+\delta^{11}+i \delta^{21}-i \delta^{22}\right)$

$E_{23} \cdot T(w)^{(x, y)}=\Theta_{\left\{f_{\alpha}, f_{\beta}\right\}}^{(x, y)}\left(E_{23}\right)(T(w))=\Theta_{f_{\beta}}^{y}\left(E_{23}\right)(T(w))$

$=E_{23}(T(w))-y E_{21} E_{31}^{-1}(T(w))$

$=\frac{\left(w_{21}-w_{31}\right)\left(w_{21}-w_{32}\right)\left(w_{21}-w_{33}\right)}{\left.w_{22}-w_{21}\right)} T\left(w+\delta^{21}\right)$

$+\frac{\left(w_{22}-w_{31}\right)\left(w_{22}-w_{32}\right)\left(w_{22}-w_{33}\right)}{w_{21}-w_{22}} T\left(w+\delta^{22}\right)$

$-y \sum_{i=1}^{d}\left(w_{21}-w_{22}+2 i-1\right) E_{21}\left(T\left(w+\delta^{11}+i \delta^{21}-(i-1) \delta^{22}\right)\right)$

$=\frac{\left(w_{21}-w_{31}\right)\left(w_{21}-w_{32}\right)\left(w_{21}-w_{33}\right)}{\left.w_{22}-w_{21}\right)} T\left(w+\delta^{21}\right)$

$+\frac{\left(w_{22}-w_{31}\right)\left(w_{22}-w_{32}\right)\left(w_{22}-w_{33}\right)}{w_{21}-w_{22}} T\left(w+\delta^{22}\right)$

$-y \sum_{i=1}^{d}\left(w_{21}-w_{22}+2 i-1\right) T\left(w+i \delta^{21}-(i-1) \delta^{22}\right)$ 
Lema 4.2.4. Sejam $x, y \in \mathbb{C} \backslash \mathbb{Z}$, tais que $x+y+\left\langle\Lambda+\rho, \alpha_{1}^{\vee}\right\rangle \notin \mathbb{Z}$ e $y+\left\langle\Lambda+\rho, \alpha_{1}^{\vee}\right\rangle \notin \mathbb{Z}$. $A$ localização de $L(\Lambda)$ com relação ao conjunto $\left\{f_{\alpha}, f_{\beta}\right\}$ e torcida pelo automorfismo $\Theta_{\left\{f_{\alpha}, f_{\beta}\right\}}^{(x, y)}$ contém um submódulo simples denso de grau d isomorfo ao $\mathfrak{s l}_{3}$-módulo de $\Gamma$-relações $V_{\mathcal{D}}\left(T\left(v_{x y}\right)\right)$, em que $T\left(v_{x y}\right)$ é a tabela genérica

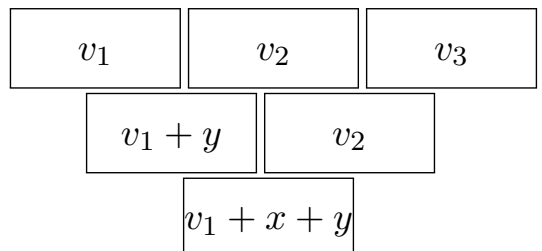

e $\mathcal{D}$ o conjunto de relações admissível definido pelo grafo:

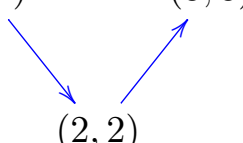

$(1,1)$

Demonstração. Consideremos o submódulo de $D_{\left\{f_{\alpha}, f_{\beta}\right\}}^{(x, y)} L(\Lambda)$ gerado por $T(w)=T\left(v-\lambda_{2} \delta^{22}\right)=$

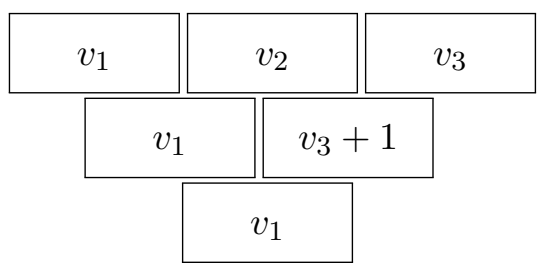

Pelo Lema 4.2.2 temos:

$$
\begin{aligned}
c_{11} \cdot T(w)^{(x, y)}= & \Theta_{\left\{f_{\alpha}, f_{\beta}\right\}}^{(x, y)}\left(c_{11}\right)(T(w))=c_{11}(T(w))+(x+y) T(w)=\left(v_{1}+x+y\right) T(w) \\
c_{21} \cdot T(w)^{(x, y)}= & \Theta_{\left\{f_{\alpha}, f_{\beta}\right\}}^{(x, y)}\left(c_{21}\right)(T(w))=c_{21}(T(w))+y T(w)=\left(v_{1}+v_{3}+2+y\right) T(w) \\
c_{22} \cdot T(w)^{(x, y)} & =\Theta_{\left\{f_{\alpha}, f_{\beta}\right\}}^{(x, y)}\left(c_{22}\right)(T(w))=\Theta_{f_{\beta}}^{y}\left(c_{22}\right)(T(w)) \\
& =\left(v_{1}^{2}+\left(v_{3}+1\right)^{2}+v_{1}+v_{3}+1+2 y v_{1}+y^{2}+y\right) T(w) \\
& =c_{22}(T(w))+y\left(2 E_{11}+y-1\right) T(w)+2 y E_{32} E_{21} E_{31}^{-1}(T(w)) \\
& =\left(v_{1}^{2}+\left(v_{3}+1\right)^{2}+v_{1}+v_{3}+1\right) T(w)+y\left(2 v_{1}+y-1\right) T(w) \\
& +2 y\left(v_{1}-v_{3}\right) E_{32}\left(T\left(w+\delta^{21}\right)\right) \\
& =\left(v_{1}^{2}+\left(v_{3}+1\right)^{2}+v_{1}+v_{3}+1+y\left(2 v_{1}+y-1\right)\right) T(w)+2 y T(w) \\
& =\left(v_{1}^{2}+\left(v_{3}+1\right)^{2}+v_{1}+v_{3}+1+2 y v_{1}+y^{2}+y\right) T(w)
\end{aligned}
$$

Por outro lado, sendo $V_{\mathcal{D}}\left(T\left(v_{x y}\right)\right)$ um módulo simples, sabemos que este pode ser gerado pela tabela $T\left(w_{x y}\right)=T\left(v_{x y}-\lambda_{2} \delta^{22}\right)=$

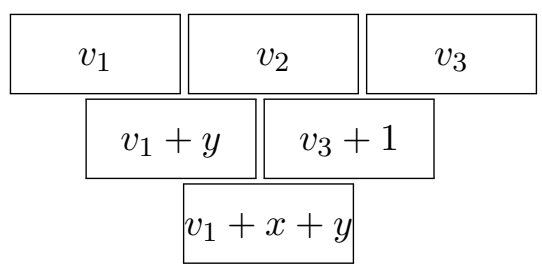


Assim pelo Teorema 3.3.10 temos

$$
\begin{aligned}
& c_{11} T\left(w_{x y}\right)=\left(v_{1}+x+y\right) T\left(w_{y}\right) \\
& c_{21} T\left(w_{x y}\right)=\left(v_{1}+y+v_{3}+2\right) T\left(w_{y}\right) \\
& c_{22} T\left(w_{x y}\right)=\left(\left(v_{1}+y\right)^{2}+\left(v_{3}+1\right)^{2}+v_{1}+y+v_{3}+1\right) T\left(w_{y}\right)
\end{aligned}
$$

Observação 4.2.5. A base de tabelas do módulo $V_{\mathcal{D}}\left(T\left(v_{x y}\right)\right)$ é:

$$
\mathcal{B}_{\mathcal{D}}\left(T\left(v_{x y}\right)\right)=L\left(\left\{\begin{array}{c}
-\lambda_{2} \leq n \leq 0 \\
l, m \in \mathbb{Z}
\end{array}\right) .\right.
$$

O próximo teorema mostra que não precisamos utilizar tabelas singulares críticas para realizar $\mathfrak{s l}_{3}$-módulos admissíveis na órbita minimal (veja Teorema 5.6 em [AFR17]).

Teorema 4.2.6. Todo $\mathfrak{s l}_{3}$-módulo admissivel simples de nível $k$ na órbita minimal $\mathbb{O}_{\text {min }}$ é um módulo limitado de $\Gamma_{\mathcal{F}}$-relações, para alguma bandeira $\mathcal{F}$.

Demonstração. Para $n=2$, todo módulo de peso máximo simples admissível no orbita minimal é um $\Gamma$-módulo de relações limitado pelo Teorema 4.1.3. Os módulos simples admissíveis na órbita minimal induzidos de um $\mathfrak{s l}_{2}$-módulo denso foram realizados no Teorema 4.1.12. Finalmente no Lema 4.2.4 realizamos os $\mathfrak{s l}_{3}$-módulos simples admissíveis densos na órbita minimal.

\subsection{Módulos simples na órbita nilpotente principal para $\mathfrak{s l}_{n+1}$}

Sejam $k$ um número admissível com o denominador $q \in \mathbb{N}$, e $\mathbb{O}_{\text {prin }}$ a órbita nilpotente principal regular, ou seja, a única órbita aberta densa no cone nilpotente $\mathcal{N}$ de $\mathfrak{g}^{*}$. Temos $\operatorname{Ann}_{U(\mathfrak{g})} L(\lambda)=$ $U(\mathfrak{g})$ ker $\chi_{\lambda}$ para todo $\lambda \in \overline{\operatorname{Pr}}_{k}^{\mathbb{O}_{\text {prin }}}$.

\subsubsection{Realização de módulos de peso máximo}

Pela Proposição 2.4.15, um elemento de $\left[\overline{\operatorname{Pr}}_{k}^{\mathbb{Q}}{ }_{\text {prin }}\right]$ para $\mathfrak{g}=\mathfrak{s l}_{n+1}$ é representado por um peso da forma

$$
\Lambda=\lambda-\frac{p}{q}(\mu+\rho)=\left(\lambda_{1}-\frac{p}{q}\left(\mu_{1}+1\right), \lambda_{2}-\frac{p}{q}\left(\mu_{2}+1\right), \ldots, \lambda_{n}-\frac{p}{q}\left(\mu_{n}+1\right)\right)
$$


em que $\lambda_{i}, \mu_{i} \in \mathbb{Z}_{\geq 0}$ para todo $i=1, \ldots, n, \lambda_{1}+\ldots+\lambda_{n}<p-n$ e $\mu_{1}+\ldots+\mu_{n}<q-n$.

Observamos que, para todo $\alpha_{i j} \in \Delta^{+}$, temos

$$
\left\langle\Lambda+\rho, \alpha_{i j}^{\vee}\right\rangle=\sum_{k=i}^{j} \lambda_{k}-\frac{p}{q}\left(\sum_{k=i}^{j} \mu_{k}+j-i+1\right)+j-i+1 .
$$

Agora, suponhamos que existam $1 \leq i \leq j \leq n$, tais que $\left\langle\Lambda+\rho, \alpha_{i j}^{\vee}\right\rangle \in \mathbb{Z}$, então

$$
q \leq \sum_{k=i}^{j} \mu_{k}+j-i+1<q
$$

Isto prova que $\Lambda$ é dominante e antidominante. Também, para cada elemento $w$ do grupo de Weyl, seja $\mu=w \cdot \Lambda$, então $\mu$ é dominante regular e antidominante.

A classificação completa de todos os módulos genéricos de Gelfand-Tsetlin simples admissíveis de nível $k$ na órbita principal, juntamente com sua construção explícita através das fórmulas de Gelfand-Tsetlin é dada no teorema seguinte.

Teorema 4.3.1 (Corolário $4.3 \mathrm{em}$ [AFR17]). Seja $\Lambda$ um $\mathfrak{g}$-peso admissível de nível $k$. Então, qualquer subquotiente simples de um módulo fortemente genérico $\left\{V(T(v)): v_{n i}=v_{i}, v_{i}-v_{i+1}=\right.$ $\left.\left\langle\Lambda+\rho, \alpha_{i}^{\vee}\right\rangle\right\}$ é um módulo simples admissivel de Gelfand-Tsetlin na órbita principal. Mais ainda, qualquer módulo simples genérico de Gelfand-Tsetlin admissivel de nível $k$ na órbita nilpotente principal com caráter central determinado por $\Lambda$ é isomórfo a um subquociente de algum módulo fortemente genérico $\left\{V(T(v)): v_{n i}=v_{i}, v_{i}-v_{i+1}=\left\langle\Lambda+\rho, \alpha_{i}^{\vee}\right\rangle\right\}$.

Na seguinte asserção descrevemos todos os módulos de peso máximo na órbita principal, a mesma segue diretamente do Teorema 4.3.1.

Proposição 4.3.2. O módulo simples de peso máximo $L(\Lambda)$ é módulo de Verma de $\Gamma$-relações. Mais ainda, todo $\mathfrak{g}$-módulo de peso máximo admissivel $L(\mu)$ de nível $k$ na órbita nilpotente principal é um $\mathfrak{g}$-módulo de Verma simples de relações.

Exemplo 4.3.3. Seja $\mathfrak{g}=\mathfrak{s l}_{3}$ e $\Lambda_{1}=\left(\lambda_{1}-\frac{p}{q}\left(\mu_{1}+1\right), \lambda_{2}-\frac{p}{q}\left(\mu_{2}+1\right)\right)$, então a órbita de Weyl de $\Lambda_{1}$ é:

- $\Lambda_{2}=s_{1} \cdot \Lambda_{1}=\left(\frac{p}{q}\left(\mu_{1}+1\right)-\lambda_{1}-2, \lambda_{1}+\lambda_{2}-\frac{p}{q}\left(\mu_{1}+\mu_{2}+2\right)+1\right)$.

- $\Lambda_{3}=s_{2} \cdot \Lambda_{1}=\left(\lambda_{1}+\lambda_{2}-\frac{p}{q}\left(\mu_{1}+\mu_{2}+2\right)+1, \frac{p}{q}\left(\mu_{2}+1\right)-\lambda_{2}-2\right)$.

- $\Lambda_{4}=s_{1} s_{2} \cdot \Lambda_{1}=\left(\frac{p}{q}\left(\mu_{1}+\mu_{2}+2\right)-\lambda_{1}-\lambda_{2}-3, \lambda_{1}-\frac{p}{q}\left(\mu_{1}+1\right)\right)$.

- $\Lambda_{5}=s_{2} s_{1} \cdot \Lambda_{1}=\left(\lambda_{2}-\frac{p}{q}\left(\mu_{2}+1\right), \frac{p}{q}\left(\mu_{1}+\mu_{2}+2\right)-\lambda_{1}-\lambda_{2}-3\right)$.

- $\Lambda_{6}=s_{1} s_{2} s_{1} \cdot \Lambda_{1}=\left(\frac{p}{q}\left(\mu_{2}+1\right)-\lambda_{2}-2, \frac{p}{q}\left(\mu_{1}+1\right)-\lambda_{1}-2\right)$.

Neste caso, temos $\left\langle\Lambda_{i}+\rho, \alpha_{1}^{\vee}\right\rangle \notin \mathbb{Z},\left\langle\Lambda_{i}+\rho, \alpha_{2}^{\vee}\right\rangle \notin \mathbb{Z}$ e $\left\langle\Lambda_{i}+\rho, \alpha_{12}^{\vee}\right\rangle \notin \mathbb{Z}$ para cada $i \in$ $\{1,2,3,4,5,6\}$. Logo, o peso $\Lambda_{i}$ é dominante regular e antidominante. Consequentemente, pelo Corolário 1.3 .18 e o Teorema 1.3.14, temos que $L\left(\Lambda_{i}\right)=M\left(\Lambda_{i}\right)$ é um módulo de $\Gamma$-relações e $\operatorname{Ann}_{U(\mathfrak{g})} L\left(\Lambda_{i}\right)=\operatorname{Ann}_{U(\mathfrak{g})} L\left(\Lambda_{1}\right)=U(\mathfrak{g})$ ker $\chi_{\Lambda_{1}}$. Isto prova que todos os $\mathfrak{s l}_{3}$-módulos de peso máximo admissíveis na órbita principal são $L\left(\Lambda_{i}\right)$ por $i=1,2,3,4,5,6$. 
Exemplo 4.3.4. Para todo $1 \leq i \leq 6$, o módulo de Verma simples $M\left(\Lambda_{i}\right)$ admite uma realização por tabelas como módulo fortemente genérico $V_{\mathcal{C}}(T(v))$, em que $T(v)$ representa a tabela

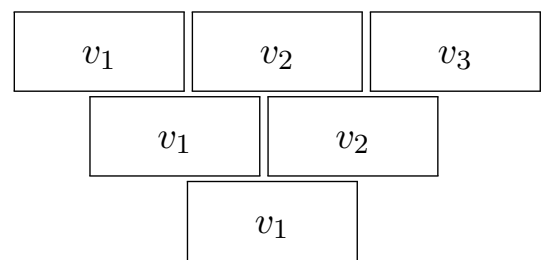

$v_{1}-v_{2}=\left\langle\Lambda_{i}+\rho, \alpha_{1}^{\vee}\right\rangle \notin \mathbb{Z}, v_{2}-v_{3}=\left\langle\Lambda_{i}+\rho, \alpha_{2}^{\vee}\right\rangle \notin \mathbb{Z}, v_{1}-v_{3}=\left\langle\Lambda_{i}+\rho, \alpha_{12}^{\vee}\right\rangle \notin \mathbb{Z}$ e $\mathcal{C}$ é o conjunto admissível de relações definido pelo grafo:

A base do módulo é formada pelo conjunto de tabelas:

$$
\mathcal{B}_{\mathcal{C}}(T(v))=L\left(\left\{\begin{array}{c}
l \leq m \leq 0 \\
n \leq 0
\end{array}\right) .\right.
$$

Exemplo 4.3.5. Seja $\mathfrak{g}=\mathfrak{s l}_{4}$ e $\Lambda_{1}=\left(\lambda_{1}-\frac{p}{q}\left(\mu_{1}+1\right), \lambda_{2}-\frac{p}{q}\left(\mu_{2}+1\right), \lambda_{3}-\frac{p}{q}\left(\mu_{3}+1\right)\right)$. Então,

- $\left\langle\Lambda_{1}+\rho, \alpha_{1}^{\vee}\right\rangle=\lambda_{1}-\frac{p}{q}\left(\mu_{1}+1\right) \notin \mathbb{Z}$

- $\left\langle\Lambda_{1}+\rho, \alpha_{2}^{\vee}\right\rangle=\lambda_{2}-\frac{p}{q}\left(\mu_{2}+1\right) \notin \mathbb{Z}$

- $\left\langle\Lambda_{1}+\rho, \alpha_{3}^{\vee}\right\rangle=\lambda_{3}-\frac{p}{q}\left(\mu_{3}+1\right) \notin \mathbb{Z}$

- $\left\langle\Lambda_{1}+\rho, \alpha_{12}^{\vee}\right\rangle=\lambda_{1}+\lambda_{2}-\frac{p}{q}\left(\mu_{1}+\mu_{2}\right)+2 \notin \mathbb{Z}$

- $\left\langle\Lambda_{1}+\rho, \alpha_{23}^{\vee}\right\rangle=\lambda_{2}+\lambda_{3}-\frac{p}{q}\left(\mu_{2}+\mu_{3}\right)+2 \notin \mathbb{Z}$

- $\left\langle\Lambda_{1}+\rho, \alpha_{13}^{\vee}\right\rangle=\lambda_{1}+\lambda_{2}+\lambda_{3}-\frac{p}{q}\left(\mu_{1}+\mu_{2}+\mu_{3}\right)+3 \notin \mathbb{Z}$

Consequentemente, $\Lambda_{1}$ é dominante regular e antidominante. Ademais, a orbita de Weyl do peso $\Lambda_{1}$ é:

a) $\Lambda_{2}=s_{1} \cdot \Lambda_{1}=\left(-\lambda_{1}+\frac{p}{q}\left(\mu_{1}+1\right)-2, \lambda_{1}+\lambda_{2}-\frac{p}{q}\left(\mu_{1}+\mu_{2}+2\right)+1, \lambda_{3}-\frac{p}{q}\left(\mu_{3}+1\right)\right)$;

b) $\Lambda_{3}=s_{2} s_{1} \cdot \Lambda_{1}=\left(\lambda_{2}-\frac{p}{q}\left(\mu_{2}+1\right),-\lambda_{1}-\lambda_{2}+\frac{p}{q}\left(\mu_{1}+\mu_{2}+2\right)-3, \lambda_{1}+\lambda_{2}+\lambda_{3}-\frac{p}{q}\left(\mu_{1}+\mu_{2}+\mu_{3}+3\right)+2\right)$;

c) $\Lambda_{4}=s_{3} s_{2} s_{1} \cdot \Lambda_{1}=\left(\lambda_{2}-\frac{p}{q}\left(\mu_{2}+1\right), \lambda_{3}-\frac{p}{q}\left(\mu_{3}+1\right),-\lambda_{1}-\lambda_{2}-\lambda_{3}+\frac{p}{q}\left(\mu_{1}+\mu_{2}+\mu_{3}+3\right)-4\right)$;

d) $\Lambda_{5}=s_{2} \cdot \Lambda_{1}=\left(\lambda_{1}+\lambda_{2}-\frac{p}{q}\left(\mu_{1}+\mu_{2}+2\right)+1,-\lambda_{2}+\frac{p}{q}\left(\mu_{2}+1\right)-2, \lambda_{2}+\lambda_{3}-\frac{p}{q}\left(\mu_{2}+\mu_{3}+2\right)+1\right)$;

e) $\Lambda_{6}=s_{3} \cdot \Lambda_{1}=\left(\lambda_{1}-\frac{p}{q}\left(\mu_{1}+1\right), \lambda_{2}+\lambda_{3}-\frac{p}{q}\left(\mu_{2}+\mu_{3}+2\right)+1,-\lambda_{3}+\frac{p}{q}\left(\mu_{3}+1\right)-2\right)$;

f) $\Lambda_{7}=s_{1} s_{2} \cdot \Lambda_{1}=\left(-\lambda_{1}-\lambda_{2}+\frac{p}{q}\left(\mu_{1}+\mu_{2}+2\right)-3, \lambda_{1}-\frac{p}{q}\left(\mu_{1}+1\right), \lambda_{2}+\lambda_{3}-\frac{p}{q}\left(\mu_{2}+\mu_{3}+2\right)+1\right)$; 
g) $\Lambda_{8}=s_{1} s_{3} \cdot \Lambda_{1}=\left(-\lambda_{1}+\frac{p}{q}\left(\mu_{1}+1\right)-2, \lambda_{1}+\lambda_{2}+\lambda_{3}-\frac{p}{q}\left(\mu_{1}+\mu_{2}+\mu_{3}+3\right)+2,-\lambda_{3}+\frac{p}{q}\left(\mu_{3}+1\right)-2\right)$;

h) $\Lambda_{9}=s_{2} s_{3} \cdot \Lambda_{1}=\left(\lambda_{1}+\lambda_{2}+\lambda_{3}-\frac{p}{q}\left(\mu_{1}+\mu_{2}+\mu_{3}+3\right)+2,-\lambda_{2}-\lambda_{3}+\frac{p}{q}\left(\mu_{2}+\mu_{3}+2\right)-3, \lambda_{2}-\frac{p}{q}\left(\mu_{2}+1\right)\right)$;

i) $\Lambda_{10}=s_{3} s_{2} \cdot \Lambda_{1}=\left(\lambda_{1}+\lambda_{2}-\frac{p}{q}\left(\mu_{1}+\mu_{2}+2\right)+1, \lambda_{3}-\frac{p}{q}\left(\mu_{3}+1\right),-\lambda_{2}-\lambda_{3}+\frac{p}{q}\left(\mu_{2}+\mu_{3}+2\right)-3\right)$;

j) $\Lambda_{11}=s_{1} s_{2} s_{1} \cdot \Lambda_{1}=\left(-\lambda_{2}+\frac{p}{q}\left(\mu_{2}+1\right)-2,-\lambda_{1}+\frac{p}{q}\left(\mu_{1}+1\right)-2, \lambda_{1}+\lambda_{2}+\lambda_{3}-\frac{p}{q}\left(\mu_{1}+\mu_{2}+\mu_{3}+3\right)+2\right)$;

k) $\Lambda_{12}=s_{1} s_{2} s_{3} \cdot \Lambda_{1}=\left(-\lambda_{1}-\lambda_{2}-\lambda_{3}+\frac{p}{q}\left(\mu_{1}+\mu_{2}+\mu_{3}+3\right)-4, \lambda_{1}-\frac{p}{q}\left(\mu_{1}+1\right), \lambda_{2}-\frac{p}{q}\left(\mu_{2}+1\right)\right)$;

l) $\Lambda_{13}=s_{1} s_{3} s_{2} \cdot \Lambda_{1}=\left(-\lambda_{1}-\lambda_{2}+\frac{p}{q}\left(\mu_{1}+\mu_{2}+2\right)-3, \lambda_{1}+\lambda_{2}+\lambda_{3}-\frac{p}{q}\left(\mu_{1}+\mu_{2}+\mu_{3}+3\right)+2,-\lambda_{2}-\right.$ $\left.\lambda_{3}+\frac{p}{q}\left(\mu_{2}+\mu_{3}+2\right)-3\right)$

m) $\Lambda_{14}=s_{2} s_{1} s_{3} \cdot \Lambda_{1}=\left(\lambda_{2}+\lambda_{3}-\frac{p}{q}\left(\mu_{2}+\mu_{3}+2\right)+1,-\lambda_{1}-\lambda_{2}-\lambda_{3}+\frac{p}{q}\left(\mu_{1}+\mu_{2}+\mu_{3}+3\right)-4, \lambda_{1}+\right.$ $\left.\lambda_{2}-\frac{p}{q}\left(\mu_{1}+\mu_{2}+2\right)+1\right)$

n) $\Lambda_{15}=s_{2} s_{3} s_{2} \cdot \Lambda_{1}=\left(\lambda_{1}+\lambda_{2}+\lambda_{3}-\frac{p}{q}\left(\mu_{1}+\mu_{2}+\mu_{3}+3\right)+2,-\lambda_{3}+\frac{p}{q}\left(\mu_{3}+1\right)-2,-\lambda_{2}+\frac{p}{q}\left(\mu_{2}+1\right)-2\right)$;

о) $\Lambda_{16}=s_{3} s_{1} s_{2} s_{1} \cdot \Lambda_{1}=\left(-\lambda_{2}+\frac{p}{q}\left(\mu_{2}+1\right)-2, \lambda_{2}+\lambda_{3}-\frac{p}{q}\left(\mu_{2}+\mu_{3}+2\right)+1,-\lambda_{1}-\lambda_{2}-\lambda_{3}+\frac{p}{q}\left(\mu_{1}+\right.\right.$ $\left.\left.\mu_{2}+\mu_{3}+3\right)-4\right)$

p) $\Lambda_{17}=s_{2} s_{1} s_{2} s_{3} \cdot \Lambda_{1}=\left(-\lambda_{2}-\lambda_{3}+\frac{p}{q}\left(\mu_{2}+\mu_{3}+2\right)-3,-\lambda_{1}+\frac{p}{q}\left(\mu_{1}+1\right)-2, \lambda_{1}+\lambda_{2}-\frac{p}{q}\left(\mu_{1}+\mu_{2}+2\right)+1\right)$;

q) $\Lambda_{18}=s_{3} s_{1} s_{2} s_{3} \cdot \Lambda_{1}=\left(-\lambda_{1}-\lambda_{2}-\lambda_{3}+\frac{p}{q}\left(\mu_{1}+\mu_{2}+\mu_{3}+3\right)-4, \lambda_{1}+\lambda_{2}-\frac{p}{q}\left(\mu_{1}+\mu_{2}+2\right)+\right.$ $\left.1,-\lambda_{2}+\frac{p}{q}\left(\mu_{2}+1\right)-2\right)$

r) $\Lambda_{19}=s_{2} s_{1} s_{3} s_{2} \cdot \Lambda_{1}=\left(\lambda_{3}-\frac{p}{q}\left(\mu_{3}+1\right),-\lambda_{1}-\lambda_{2}-\lambda_{3}+\frac{p}{q}\left(\mu_{1}+\mu_{2}+\mu_{3}+3\right)-4, \lambda_{1}-\frac{p}{q}\left(\mu_{1}+1\right)\right)$;

s) $\Lambda_{20}=s_{3} s_{2} s_{1} s_{3} \cdot \Lambda_{1}=\left(\lambda_{2}+\lambda_{3}-\frac{p}{q}\left(\mu_{2}+\mu_{3}+2\right)+1,-\lambda_{3}+\frac{p}{q}\left(\mu_{3}+1\right)-2,-\lambda_{1}-\lambda_{2}+\frac{p}{q}\left(\mu_{1}+\mu_{2}+2\right)-3\right)$;

t) $\Lambda_{21}=s_{2} s_{3} s_{1} s_{2} s_{1} \cdot \Lambda_{1}=\left(\lambda_{2}-\frac{p}{q}\left(\mu_{2}+1\right),-\lambda_{2}-\lambda_{3}+\frac{p}{q}\left(\mu_{2}+\mu_{3}+2\right)-3,-\lambda_{1}+\frac{p}{q}\left(\mu_{1}+1\right)-2\right)$;

u) $\Lambda_{22}=s_{3} s_{1} s_{2} s_{1} s_{3} \cdot \Lambda_{1}=\left(-\lambda_{2}-\lambda_{3}+\frac{p}{q}\left(\mu_{2}+\mu_{3}+2\right)-3, \lambda_{2}-\frac{p}{q}\left(\mu_{2}+1\right),-\lambda_{1}-\lambda_{2}+\frac{p}{q}\left(\mu_{1}+\mu_{2}+2\right)-3\right)$;

v) $\Lambda_{23}=s_{2} s_{3} s_{1} s_{2} s_{3} \cdot \Lambda_{1}=\left(-\lambda_{3}+\frac{p}{q}\left(\mu_{3}+1\right)-2,-\lambda_{1}-\lambda_{2}+\frac{p}{q}\left(\mu_{1}+\mu_{2}+2\right)-3, \lambda_{1}-\frac{p}{q}\left(\mu_{1}+1\right)\right)$;

w) $\Lambda_{24}=s_{1} s_{2} s_{3} s_{1} s_{2} s_{1} \cdot \Lambda_{1}=\left(-\lambda_{2}+\frac{p}{q}\left(\mu_{2}+1\right)-2,-\lambda_{3}+\frac{p}{q}\left(\mu_{3}+1\right)-2,-\lambda_{1}+\frac{p}{q}\left(\mu_{1}+1\right)-2\right)$.

Lembramos que $\lambda_{i} \in \mathbb{Z}_{\geq 0}$ e $\mu_{i} \in \mathbb{Z}_{\geq 0}$ para cada $i=1,2,3$. Logo, dados $w \in W=S_{4}$ e $\alpha_{i j} \in \Delta^{+}$ com $1 \leq i \leq j \leq 3$, temos $\left\langle\mu+\rho, \alpha_{i j}^{\vee}\right\rangle \notin \mathbb{Z}$, em que $\mu=w \cdot \Lambda_{1}$, assim $\mu$ é dominante regular e antidominante. Consequentemente, para cada $1 \leq i \leq 24, L\left(\Lambda_{i}\right)$ é um $\mathfrak{s l}_{4}$-módulo de Verma admissível de relações na órbita principal (veja Corolários 1.3.18 e 3.4.12).

Observação 4.3.6. Todos esses módulos de peso máximo têm espaços de peso de dimensão finita, porém ilimitada e correspondem a tabelas fortemente genéricas, isto é, para cada $1 \leq i \leq 24$ o módulo de Verma $M\left(\Lambda_{i}\right)$ admite uma realização por tabelas como um $\mathfrak{s l}_{4}$-módulo simples $V_{\mathcal{C}}(T(v))$, em que $v_{1}-v_{2}=\left\langle\Lambda_{i}+\rho, \alpha_{1}^{\vee}\right\rangle, v_{2}-v_{3}=\left\langle\Lambda_{i}+\rho, \alpha_{2}^{\vee}\right\rangle, v_{3}-v_{4}=\left\langle\Lambda_{i}+\rho, \alpha_{3}^{\vee}\right\rangle, v_{1}+v_{2}+v_{3}+v_{4}=-6$ e $T(v)$ é a tabela fortemente genérica (veja Exemplo 3.4.4)

\begin{tabular}{|l|l|l|l|l|}
\hline$v_{1}$ & & $v_{2}$ & $v_{3}$ & $v_{4}$ \\
\hline$v_{1}$ & $v_{2}$ & $v_{3}$ \\
\hline
\end{tabular}


$v_{1}$ com conjunto de relações $\mathcal{C}$ definidas pelo seguinte grafo:

$(2,1)$

Uma base para este módulo é dada pelo conjunto de tabelas

$$
\mathcal{B}_{\mathcal{C}}(T(v))=L\left(\left\{\begin{array}{c}
l \leq m \leq r \leq 0 \\
n \leq s \leq 0
\end{array}\right) .\right.
$$

\subsubsection{Realização dos $\mathfrak{s l}_{2}$-módulos induzidos}

No teorema a seguir realizaremos todos os $\mathfrak{s l}_{n+1}$-módulos admissíveis simples induzidos de $\mathfrak{s l}_{2}$ na órbita nilpotente principal a menos da ação do grupo de Weyl.

Observação 4.3.7. $\mathcal{O}_{\min }=\mathcal{O}_{\text {prin }}=\mathcal{O}_{2}$ para $\mathfrak{g}=\mathfrak{s l}_{2}$.

Teorema 4.3.8. Seja $n>1, \gamma \in \mathbb{C}, \sigma=\left(\sigma_{1}, \ldots, \sigma_{n}\right) \in \mathfrak{h}^{*}$, tal que $V \simeq V\left(\gamma, \sigma_{1}\right)$ é um $\mathfrak{s l}_{2}$ módulo de peso simples denso e $\sigma_{i}=\left\langle\sigma, \alpha_{i}^{\vee}\right\rangle, i=1, \ldots, n$. Seja $L(\gamma, \sigma)=L_{\mathfrak{p}}^{\mathfrak{g}}(\{\alpha\}, V)$. Então $L(\gamma, \sigma)$ é admissível de nível $k$ na órbita principal se, e somente se, $\sigma_{1}=\lambda_{1}-\frac{p}{q}\left(\mu_{1}+1\right)+2 x$, $\sigma_{2}=\lambda_{2}-\frac{p}{q}\left(\mu_{2}+1\right)-x, \sigma_{j}=\lambda_{j}-\frac{p}{q}\left(\mu_{j}+1\right)$ para todos $j=3, \ldots, n$ e $\gamma=\left(\lambda_{1}-\frac{p}{q}\left(\mu_{1}+1\right)+1\right)^{2}$ com $\lambda_{i}, \mu_{i} \in \mathbb{Z}_{\geq 0}$ para todo $i=1, \ldots, n$, de modo que $\lambda_{1}+\ldots+\lambda_{n}<p-n, \mu_{1}+\ldots+\mu_{n}<q-n$, $x \in \mathbb{C} \backslash \mathbb{Z}$ e $x-\frac{p}{q}\left(\mu_{1}+1\right) \notin \mathbb{Z}$.

Demonstração. Podemos realizar uma prova semelhante à demostração do Teorema 4.1.12, em que $L\left(u_{1}, u_{2}, v_{1}, \ldots, v_{n}\right)$ denota o $\mathfrak{g}$-módulo simples ilimitado de relações, descrito em Teorema 3.5.9.

Observação 4.3.9. O Teorema 4.3 .8 fornece uma classificação completa de todos os módulos de peso simples admissíveis na órbita nilpotente principal induzidos de $\mathfrak{s l}_{2}$ (veja Observação 6.6 em [AFR17]).

Nosso último resultado prova que existem $\mathfrak{s l}_{4}$-módulos simples de relações na órbita principal com espaços de peso de dimensão finita induzidos de $\mathfrak{s l}_{2} \oplus \mathfrak{s l}_{2}$-módulos densos simples. 
Teorema 4.3.10. Seja $\mathfrak{g}=\mathfrak{s l}_{4}$ e um elemento de $\Lambda \in\left[\overline{\operatorname{Pr}}_{k}^{\mathbb{O}}{ }_{\text {prin }}\right]$. Para cada par de números complexos $x, y \notin \mathbb{Z}$ satisfazendo $x+\left\langle\Lambda+\rho, \alpha_{1}^{\vee}\right\rangle \notin \mathbb{Z}, y-\left\langle\Lambda+\rho, \alpha_{2}^{\vee}\right\rangle, y+\left\langle\Lambda+\rho, \alpha_{3}^{\vee}\right\rangle \notin \mathbb{Z} e$ $y-\left\langle\Lambda+\rho, \alpha_{12}^{\vee}\right\rangle \notin \mathbb{Z}$, escolha-se números complexos $v_{i}$, para $i=1,2,3,4$, tais que $v_{1}-v_{2}=$ $\left\langle\Lambda+\rho, \alpha_{1}^{\vee}\right\rangle, v_{2}-v_{3}=\left\langle\Lambda+\rho, \alpha_{2}^{\vee}\right\rangle, v_{3}-v_{4}=\left\langle\Lambda+\rho, \alpha_{3}^{\vee}\right\rangle$ e defina-se $u_{0}=v_{1}+x$ e $u_{1}=v_{3}+y$. Então, $V_{\mathcal{C}}(T(v))$ é um $\mathfrak{g}$-módulo simples de relações admissível na órbita principal induzido de um $\mathfrak{s l}_{2} \oplus \mathfrak{s l}_{2}$-módulo denso simples, em que $T(v)$ é a tabela genérica

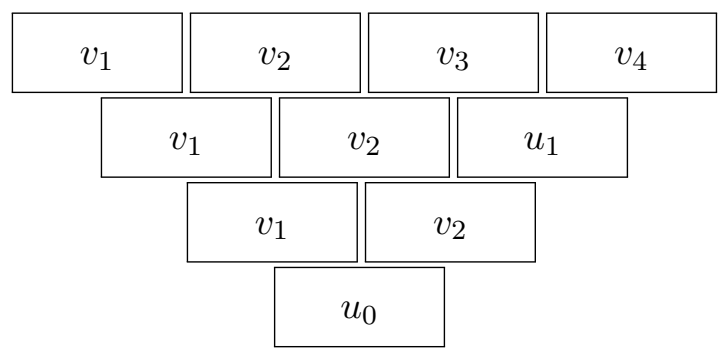

$e \mathcal{C}$ é o conjunto de relações com grafo associado

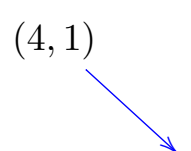

$(3,1)$

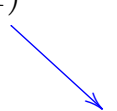

$(2,1)$

$(4,2)$

$(3,2)$

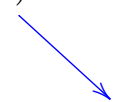

$(2,2)$

$(1,1)$

Demonstração. Por hipótese $\Lambda \in\left[\overline{P r}_{k}^{\mathbb{O}_{\text {prin }}}\right]$, então $\left\langle\Lambda+\rho, \alpha_{i j}^{\vee}\right\rangle \notin \mathbb{Z}$, para cada $1 \leq i \leq j \leq 3$, logo $v_{i}-v_{j} \notin \mathbb{Z}$ para cada para cada $1 \leq i<j \leq 4$. Também, pela escolha de $x$ e $y$, temos que $u_{0}-v_{1}=$ $x \notin \mathbb{Z}, u_{0}-v_{2}=\left\langle\Lambda+\rho, \alpha_{1}^{\vee}\right\rangle+x \notin \mathbb{Z}, u_{1}-v_{1}=y-\left\langle\Lambda+\rho, \alpha_{12}^{\vee}\right\rangle \notin \mathbb{Z}, u_{1}-v_{2}=y-\left\langle\Lambda+\rho, \alpha_{2}^{\vee}\right\rangle \notin \mathbb{Z}$, $u_{1}-v_{3}=y \notin \mathbb{Z}$ e $u_{1}-v_{4}=y+\left\langle\Lambda+\rho, \alpha_{3}^{\vee}\right\rangle \notin \mathbb{Z}$. Então pelo Lema 3.8.7, $V_{\mathcal{C}}(T(v))$ é um $\mathfrak{g}$-módulo simples de relações induzido de um $\mathfrak{s l}_{2} \oplus \mathfrak{s l}_{2}$-módulo denso simples. Finalmente, pelo Teorema 4.3 .1 $V_{\mathcal{C}}(T(v))$ é um g-módulo admissível na órbita principal.

Observação 4.3.11. A base de $V_{\mathcal{C}}(T(v))$ é o conjunto de tabelas

$$
\mathcal{B}_{\mathcal{C}}(T(v))=L\left(\left\{\begin{array}{l}
m \leq r \leq 0 \\
n \leq s \leq 0 \\
l, t \in \mathbb{Z}
\end{array}\right) .\right.
$$




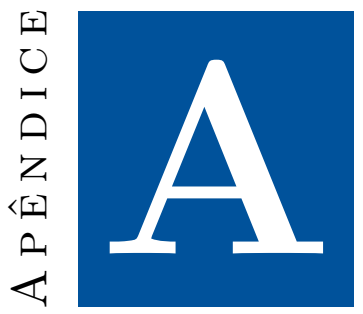

\section{Grupo de Weyl}

Neste apartado vamos recordar a definição do grupo de Weyl e descreveremos este por extensão para os álgebra $\mathfrak{s l}_{3}$ e $\mathfrak{s l}_{4}$. Assumimos que o leitor possui uma noção básica sobre esta teoria a qual pode ser consultado em [Dix77, Hum80, Mar10, Car05].

Para um sistema de raízes $\Delta$ associado a uma álgebra de Lie $\mathfrak{g}$, com subálgebra de Cartan $\mathfrak{h}$, há um grupo de operadores lineares de $\mathfrak{h}^{*}$ que revela uma bela geometria e simetria no sistema de raízes $\Delta$, que é o chamado grupo de Weyl. Relembraremos a sua construção e principais propriedades do grupo de Weyl nesta subseção.

Para cada $\alpha \in \Delta$ consideremos o operador linear $s_{\alpha}: \mathfrak{h}^{*} \rightarrow \mathfrak{h}^{*}$ que é a reflexão em torno de um hiperplano ortogonal a $\alpha$ dada por:

$$
s_{\alpha}(\lambda)=\lambda-2 \frac{(\lambda, \alpha)}{(\alpha, \alpha)} \alpha .
$$

De posse desses operadores, temos então a definição do grupo de Weyl.

Definição A.0.1. O grupo $W$ gerado pelas aplicações $s_{\alpha}$, com $\alpha \in \Delta$, é chamado de grupo de Weyl de g.

O grupo de Weyl goza de inúmeras propriedades interessantes. Aqui listaremos as mais relevantes (cujas provas podem ser encontradas, por exemplo, em [Hum80]). A primeira delas é que o grupo de Weyl permuta as raízes, ou seja, se $w \in W$, então $w(\alpha) \in \Delta$, sempre que $\alpha \in \Delta$, o que nos permite concluir que $W$ é um grupo finito, visto que é um subgrupo do grupo de permutações do conjunto finito $\Delta$. Outra propriedade relevante é que dada qualquer raiz $\alpha \in \Delta$, existe uma raiz simples $\beta \pi$ e um elemento $w \in W$ tais que $\alpha=w(\beta)$, isto é, $\Delta=W(\pi)$. Em particular, se conhecermos das raízes simples, podemos obter todo o sistema de raízes $\Delta$ por sucessivas reflexões.

A seguir descrevemos explicitamente a órbita de Weyl de um peso para $\mathfrak{s l}_{3}$ e $\mathfrak{s l}_{4}$, também apresentamos a Ordem de Bruhat e as Órbitas de Weyl, respectivamente. As definições e teoria geral podem ser encontradas em [BB05].

Seja $\mathfrak{g}=\mathfrak{s l}_{3}$. Seja $\lambda=\left(\lambda_{1}, \lambda_{2}\right)=\lambda_{1} \omega_{1}+\lambda_{2} \omega_{2}$, então a órbita do grupo de Weyl de $\lambda$ é: 


\begin{tabular}{|c|c|c|}
\hline & $w$ & $w \lambda$ \\
\hline id & id & $\left(\lambda_{1}, \lambda_{2}\right)$ \\
\hline$(1)$ & $s_{1}$ & $\left(-\lambda_{1}, \lambda_{1}+\lambda_{2}\right)$ \\
\hline$(2)$ & $s_{2}$ & $\left(\lambda_{1}+\lambda_{2},-\lambda_{2}\right)$ \\
\hline$(12)$ & $s_{1} s_{2}$ & $\left(-\lambda_{1}-\lambda_{2}, \lambda_{1}\right)$ \\
\hline$(21)$ & $s_{2} s_{1}$ & $\left(\lambda_{2},-\lambda_{1}-\lambda_{2}\right)$ \\
\hline$(121)$ & $s_{1} s_{2} s_{1}$ & $\left(-\lambda_{2},-\lambda_{1}\right)$ \\
\hline
\end{tabular}

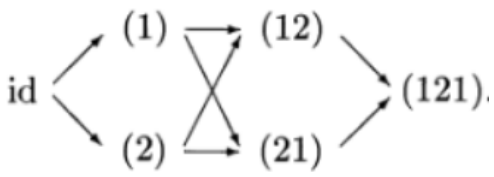

Figura A.1: Ordem de Bruhat $S_{3}$.

Consequentemente,

\begin{tabular}{|c|c|c|}
\hline & $w$ & $w \cdot \lambda$ \\
\hline id & id & $\left(\lambda_{1}, \lambda_{2}\right)$ \\
\hline$(1)$ & $s_{1}$ & $\left(-\lambda_{1}-2, \lambda_{1}+\lambda_{2}+1\right)$ \\
\hline$(2)$ & $s_{2}$ & $\left(\lambda_{1}+\lambda_{2}+1,-\lambda_{2}-2\right)$ \\
\hline$(12)$ & $s_{1} s_{2}$ & $\left(-\lambda_{1}-\lambda_{2}-3, \lambda_{1}\right)$ \\
\hline$(21)$ & $s_{2} s_{1}$ & $\left(\lambda_{2},-\lambda_{1}-\lambda_{2}-3\right)$ \\
\hline$(121)$ & $s_{1} s_{2} s_{1}$ & $\left(-\lambda_{2}-2,-\lambda_{1}-2\right)$ \\
\hline
\end{tabular}

\begin{tabular}{|c|c|c|}
\hline & $w$ & $w \lambda$ \\
\hline$(123)$ & id & $\left\{\alpha_{1}, \alpha_{2}\right\}$ \\
\hline$(213)$ & $s_{1}$ & $\left\{-\alpha_{1}, \alpha_{1}+\alpha_{2}\right\}$ \\
\hline$(132)$ & $s_{2}$ & $\left\{\alpha_{1}+\alpha_{2},-\alpha_{2}\right\}$ \\
\hline$(231)$ & $s_{1} s_{2}$ & $\left\{\alpha_{2},-\alpha_{1}-\alpha_{2}\right\}$ \\
\hline$(312)$ & $s_{2} s_{1}$ & $\left\{-\alpha_{1}-\alpha_{2}, \alpha_{1}\right\}$ \\
\hline$(321)$ & $s_{1} s_{2} s_{1}$ & $\left\{-\alpha_{2},-\alpha_{1}\right\}$ \\
\hline
\end{tabular}

Caso I: $w=s_{1}$

Se $\lambda=\lambda_{1} \omega_{1}+\lambda_{2} \omega_{2}$ então $\mu:=w \lambda=-\lambda_{1} \omega_{1}+\left(\lambda_{1}+\lambda_{2}\right) \omega_{2}$. Logo

- $\omega_{1}^{\prime}:=w \omega_{1}=-\omega_{1}+\omega_{2}$

- $\omega_{2}^{\prime}:=w \omega_{2}=\omega_{2}$

- $\rho^{\prime}:=w \rho=-\omega_{1}+2 \omega_{2}=\omega_{1}^{\prime}+\omega_{2}^{\prime}$

- $\beta_{1}:=w \alpha_{1}=-\alpha_{1}=2 \omega_{1}^{\prime}-\omega_{2}^{\prime}$

- $\beta_{2}:=w \alpha_{2}=\alpha_{12}=-\omega_{1}^{\prime}+2 \omega_{2}^{\prime}$ 
- $\beta_{12}:=w \alpha_{12}=\alpha_{2}=\beta_{1}+\beta_{2}=\omega_{1}^{\prime}+\omega_{2}^{\prime}$

Portanto, $\mu=\lambda_{1} \omega_{1}^{\prime}+\lambda_{2} \omega_{2}^{\prime} \mathrm{e}$

- $\left\langle\mu+\rho^{\prime}, \beta_{1}^{\vee}\right\rangle=\lambda_{1}+1=\left\langle\lambda+\rho, \alpha_{1}^{\vee}\right\rangle$

- $\left\langle\mu+\rho^{\prime}, \beta_{2}^{\vee}\right\rangle=\lambda_{2}+1=\left\langle\lambda+\rho, \alpha_{2}^{\vee}\right\rangle$

- $\left\langle\mu+\rho^{\prime}, \beta_{12}^{\vee}\right\rangle=\lambda_{1}+\lambda_{2}+2=\left\langle\lambda+\rho, \alpha_{12}^{\vee}\right\rangle$

Caso II: $w=s_{2}$

Se $\lambda=\lambda_{1} \omega_{1}+\lambda_{2} \omega_{2}$ então $\mu:=w \lambda=\left(\lambda_{1}+\lambda_{2}\right) \omega_{1}-\lambda_{2} \omega_{2}$. Assim,

- $\omega_{1}^{\prime}:=w \omega_{1}=\omega_{1}$

- $\omega_{2}^{\prime}:=w \omega_{2}=\omega_{1}-\omega_{2}$

- $\rho^{\prime}:=w \rho=2 \omega_{1}-\omega_{2}=\omega_{1}^{\prime}+\omega_{2}^{\prime}$

- $\beta_{1}:=w \alpha_{1}=\alpha_{1}+\alpha_{2}=2 \omega_{1}^{\prime}-\omega_{2}^{\prime}$

- $\beta_{2}:=w \alpha_{2}=-\alpha_{2}=-\omega_{1}^{\prime}+2 \omega_{2}^{\prime}$

- $\beta_{12}:=w \alpha_{12}=\alpha_{1}=\beta_{1}+\beta_{2}=\omega_{1}^{\prime}+\omega_{2}^{\prime}$

Portanto, $\mu=\lambda_{1} \omega_{1}^{\prime}+\lambda_{2} \omega_{2}^{\prime}$ e

- $\left\langle\mu+\rho^{\prime}, \beta_{1}^{\vee}\right\rangle=\lambda_{1}+1=\left\langle\lambda+\rho, \alpha_{1}^{\vee}\right\rangle$

- $\left\langle\mu+\rho^{\prime}, \beta_{2}^{\vee}\right\rangle=\lambda_{2}+1=\left\langle\lambda+\rho, \alpha_{2}^{\vee}\right\rangle$

- $\left\langle\mu+\rho^{\prime}, \beta_{12}^{\vee}\right\rangle=\lambda_{1}+\lambda_{2}+2=\left\langle\lambda+\rho, \alpha_{12}^{\vee}\right\rangle$

Caso III: $w=s_{1} s_{2}$

Se $\lambda=\lambda_{1} \omega_{1}+\lambda_{2} \omega_{2}$ então $\mu:=w \lambda=\lambda_{2} \omega_{1}-\left(\lambda_{1}+\lambda_{2}\right) \omega_{2}$. Logo,

- $\omega_{1}^{\prime}:=w \omega_{1}=-\omega_{2}$

- $\omega_{2}^{\prime}:=w \omega_{2}=\omega_{1}-\omega_{2}$

- $\rho^{\prime}:=w \rho=\omega_{1}-2 \omega_{2}=\omega_{1}^{\prime}+\omega_{2}^{\prime}$

- $\beta_{1}:=w \alpha_{1}=-\alpha_{1}-\alpha_{2}=2 \omega_{1}^{\prime}-\omega_{2}^{\prime}$

- $\beta_{2}:=w \alpha_{2}=\alpha_{1}=-\omega_{1}^{\prime}+2 \omega_{2}^{\prime}$

- $\beta_{12}:=w \alpha_{12}=-\alpha_{2}=\beta_{1}+\beta_{2}=\omega_{1}^{\prime}+\omega_{2}^{\prime}$

Portanto, $\mu=\lambda_{1} \omega_{1}^{\prime}+\lambda_{2} \omega_{2}^{\prime} \mathrm{e}$

- $\left\langle\mu+\rho^{\prime}, \beta_{1}^{\vee}\right\rangle=\lambda_{1}+1=\left\langle\lambda+\rho, \alpha_{1}^{\vee}\right\rangle$

- $\left\langle\mu+\rho^{\prime}, \beta_{2}^{\vee}\right\rangle=\lambda_{2}+1=\left\langle\lambda+\rho, \alpha_{2}^{\vee}\right\rangle$ 
- $\left\langle\mu+\rho^{\prime}, \beta_{12}^{\vee}\right\rangle=\lambda_{1}+\lambda_{2}+2=\left\langle\lambda+\rho, \alpha_{12}^{\vee}\right\rangle$

Caso IV: $w=s_{2} s_{1}$

Se $\lambda=\lambda_{1} \omega_{1}+\lambda_{2} \omega_{2}$ então $\mu:=w \lambda=-\left(\lambda_{1}+\lambda_{2}\right) \omega_{1}+\lambda_{1} \omega_{2}$. Em consequência,

- $\omega_{1}^{\prime}:=w \omega_{1}=-\omega_{1}+\omega_{2}$

- $\omega_{2}^{\prime}:=w \omega_{2}=-\omega_{1}$

- $\rho^{\prime}:=w \rho=-2 \omega_{1}+\omega_{2}=\omega_{1}^{\prime}+\omega_{2}^{\prime}$

- $\beta_{1}:=w \alpha_{1}=\alpha_{2}=2 \omega_{1}^{\prime}-\omega_{2}^{\prime}$

- $\beta_{2}:=w \alpha_{2}=-\alpha_{1}-\alpha_{2}=-\omega_{1}^{\prime}+2 \omega_{2}^{\prime}$

- $\beta_{12}:=w \alpha_{12}=-\alpha_{1}=\beta_{1}+\beta_{2}=\omega_{1}^{\prime}+\omega_{2}^{\prime}$

Portanto, $\mu=\lambda_{1} \omega_{1}^{\prime}+\lambda_{2} \omega_{2}^{\prime} \mathrm{e}$

- $\left\langle\mu+\rho^{\prime}, \beta_{1}^{\vee}\right\rangle=\lambda_{1}+1=\left\langle\lambda+\rho, \alpha_{1}^{\vee}\right\rangle$

- $\left\langle\mu+\rho^{\prime}, \beta_{2}^{\vee}\right\rangle=\lambda_{2}+1=\left\langle\lambda+\rho, \alpha_{2}^{\vee}\right\rangle$

- $\left\langle\mu+\rho^{\prime}, \beta_{12}^{\vee}\right\rangle=\lambda_{1}+\lambda_{2}+2=\left\langle\lambda+\rho, \alpha_{12}^{\vee}\right\rangle$

Caso V: $w=s_{1} s_{2} s_{1}$

Se $\lambda=\lambda_{1} \omega_{1}+\lambda_{2} \omega_{2}$ então $\mu:=w \lambda=-\lambda_{2} \omega_{1}-\lambda_{1} \omega_{2}$. Logo,

- $\omega_{1}^{\prime}:=w \omega_{1}=-\omega_{2}$

- $\omega_{2}^{\prime}:=w \omega_{2}=-\omega_{1}$

- $\rho^{\prime}:=w \rho=-\omega_{2}-\omega_{1}=\omega_{1}^{\prime}+\omega_{2}^{\prime}$

- $\beta_{1}:=w \alpha_{1}=-\alpha_{2}=2 \omega_{1}^{\prime}-\omega_{2}^{\prime}$

- $\beta_{2}:=w \alpha_{2}=-\alpha_{1}=-\omega_{1}^{\prime}+2 \omega_{2}^{\prime}$

- $\beta_{12}:=w \alpha_{12}=-\alpha_{1}-\alpha_{2}=\beta_{1}+\beta_{2}=\omega_{1}^{\prime}+\omega_{2}^{\prime}$

Portanto, $\mu=\lambda_{1} \omega_{1}^{\prime}+\lambda_{2} \omega_{2}^{\prime} \mathrm{e}$

- $\left\langle\mu+\rho^{\prime}, \beta_{1}^{\vee}\right\rangle=\lambda_{1}+1=\left\langle\lambda+\rho, \alpha_{1}^{\vee}\right\rangle$

- $\left\langle\mu+\rho^{\prime}, \beta_{2}^{\vee}\right\rangle=\lambda_{2}+1=\left\langle\lambda+\rho, \alpha_{2}^{\vee}\right\rangle$

- $\left\langle\mu+\rho^{\prime}, \beta_{12}^{\vee}\right\rangle=\lambda_{1}+\lambda_{2}+2=\left\langle\lambda+\rho, \alpha_{12}^{\vee}\right\rangle$

$\mathfrak{g}=\mathfrak{s l}_{4}$. Seja $\lambda=\left(\lambda_{1}, \lambda_{2}, \lambda_{3}\right)=\lambda_{1} \omega_{1}+\lambda_{2} \omega_{2}+\lambda_{3} \omega_{3}$, então a órbita do grupo de Weyl de $\lambda$ é: 


\begin{tabular}{|c|c|c|}
\hline & $w$ & $w \lambda$ \\
\hline id & id & $\left(\lambda_{1}, \lambda_{2}, \lambda_{3}\right)$ \\
\hline$(1)$ & $s_{1}$ & $\left(-\lambda_{1}, \lambda_{1}+\lambda_{2}, \lambda_{3}\right)$ \\
\hline$(2)$ & $s_{2}$ & $\left(\lambda_{1}+\lambda_{2},-\lambda_{2}, \lambda_{2}+\lambda_{3}\right)$ \\
\hline$(3)$ & $s_{3}$ & $\left(\lambda_{1}, \lambda_{2}+\lambda_{3},-\lambda_{3}\right)$ \\
\hline$(12)$ & $s_{1} s_{2}$ & $\left(-\lambda_{1}-\lambda_{2}, \lambda_{1}, \lambda_{2}+\lambda_{3}\right)$ \\
\hline$(21)$ & $s_{2} s_{1}$ & $\left(\lambda_{2},-\lambda_{1}-\lambda_{2}, \lambda_{1}+\lambda_{2}+\lambda_{3}\right)$ \\
\hline (13) & $s_{1} s_{3}$ & $\left(-\lambda_{1}, \lambda_{1}+\lambda_{2}+\lambda_{3},-\lambda_{3}\right)$ \\
\hline$(23)$ & $s_{2} s_{3}$ & $\left(\lambda_{1}+\lambda_{2}+\lambda_{3},-\lambda_{2}-\lambda_{3}, \lambda_{2}\right)$ \\
\hline$(32)$ & $s_{3} s_{2}$ & $\left(\lambda_{1}+\lambda_{2}, \lambda_{3},-\lambda_{2}-\lambda_{3}\right)$ \\
\hline$(123)$ & $s_{1} s_{2} s_{3}$ & $\left(-\lambda_{1}-\lambda_{2}-\lambda_{3}, \lambda_{1}, \lambda_{2}\right)$ \\
\hline$(121)$ & $s_{1} s_{2} s_{1}$ & $\left(-\lambda_{2},-\lambda_{1}, \lambda_{1}+\lambda_{2}+\lambda_{3}\right)$ \\
\hline$(213)$ & $s_{2} s_{1} s_{3}$ & $\left(\lambda_{2}+\lambda_{3},-\lambda_{1}-\lambda_{2}-\lambda_{3}, \lambda_{1}+\lambda_{2}\right)$ \\
\hline$(132)$ & $s_{1} s_{3} s_{2}$ & $\left(-\lambda_{1}-\lambda_{2}, \lambda_{1}+\lambda_{2}+\lambda_{3},-\lambda_{2}-\lambda_{3}\right)$ \\
\hline$(321)$ & $s_{3} s_{2} s_{1}$ & $\left(\lambda_{2}, \lambda_{3},-\lambda_{1}-\lambda_{2}-\lambda_{3}\right)$ \\
\hline$(232)$ & $s_{2} s_{3} s_{2}$ & $\left(\lambda_{1}+\lambda_{2}+\lambda_{3},-\lambda_{3},-\lambda_{2}\right)$ \\
\hline$(1213)$ & $s_{1} s_{2} s_{1} s_{3}$ & $\left(-\lambda_{2}-\lambda_{3},-\lambda_{1}, \lambda_{1}+\lambda_{2}\right)$ \\
\hline$(1232)$ & $s_{1} s_{2} s_{3} s_{2}$ & $\left(-\lambda_{1}-\lambda_{2}-\lambda_{3}, \lambda_{1}+\lambda_{2},-\lambda_{2}\right)$ \\
\hline$(2132)$ & $s_{2} s_{1} s_{3} s_{2}$ & $\left(\lambda_{3},-\lambda_{1}-\lambda_{2}-\lambda_{3}, \lambda_{1}\right)$ \\
\hline$(1321)$ & $s_{1} s_{3} s_{2} s_{1}$ & $\left(-\lambda_{2}, \lambda_{2}+\lambda_{3},-\lambda_{1}-\lambda_{2}-\lambda_{3}\right)$ \\
\hline$(2321)$ & $s_{2} s_{3} s_{2} s_{1}$ & $\left(\lambda_{2}+\lambda_{3},-\lambda_{3},-\lambda_{1}-\lambda_{2}\right)$ \\
\hline$(12132)$ & $s_{1} s_{2} s_{1} s_{3} s_{2}$ & $\left(-\lambda_{3},-\lambda_{1}-\lambda_{2}, \lambda_{1}\right)$ \\
\hline$(12321)$ & $s_{1} s_{2} s_{3} s_{2} s_{1}$ & $\left(-\lambda_{2}-\lambda_{3}, \lambda_{2},-\lambda_{1}-\lambda_{2}\right)$ \\
\hline$(21321)$ & $s_{2} s_{1} s_{3} s_{2} s_{1}$ & $\left(\lambda_{3},-\lambda_{2}-\lambda_{3},-\lambda_{1}\right)$ \\
\hline$(121321)$ & $s_{1} s_{2} s_{1} s_{3} s_{2} s_{1}$ & $\left(-\lambda_{3},-\lambda_{2},-\lambda_{1}\right)$ \\
\hline
\end{tabular}

\section{Consequentemente,}




\begin{tabular}{|c|c|c|}
\hline & $w$ & $w \cdot \lambda$ \\
\hline id & id & $\left(\lambda_{1}, \lambda_{2}, \lambda_{3}\right)$ \\
\hline$(1)$ & $s_{1}$ & $\left(-\lambda_{1}-2, \lambda_{1}+\lambda_{2}+1, \lambda_{3}\right)$ \\
\hline$(2)$ & $s_{2}$ & $\left(\lambda_{1}+\lambda_{2}+1,-\lambda_{2}-2, \lambda_{2}+\lambda_{3}+1\right)$ \\
\hline$(3)$ & $s_{3}$ & $\left(\lambda_{1}, \lambda_{2}+\lambda_{3}+1,-\lambda_{3}-2\right)$ \\
\hline$(12)$ & $s_{1} s_{2}$ & $\left(-\lambda_{1}-\lambda_{2}-3, \lambda_{1}, \lambda_{2}+\lambda_{3}+1\right)$ \\
\hline$(21)$ & $s_{2} s_{1}$ & $\left(\lambda_{2},-\lambda_{1}-\lambda_{2}-3, \lambda_{1}+\lambda_{2}+\lambda_{3}+2\right)$ \\
\hline$(13)$ & $s_{1} s_{3}$ & $\left(-\lambda_{1}-2, \lambda_{1}+\lambda_{2}+\lambda_{3}+2,-\lambda_{3}-2\right)$ \\
\hline$(23)$ & $s_{2} s_{3}$ & $\left(\lambda_{1}+\lambda_{2}+\lambda_{3}+2,-\lambda_{2}-\lambda_{3}-3, \lambda_{2}\right)$ \\
\hline$(32)$ & $s_{3} s_{2}$ & $\left(\lambda_{1}+\lambda_{2}+1, \lambda_{3},-\lambda_{2}-\lambda_{3}-3\right)$ \\
\hline$(123)$ & $s_{1} s_{2} s_{3}$ & $\left(-\lambda_{1}-\lambda_{2}-\lambda_{3}-4, \lambda_{1}, \lambda_{2}\right)$ \\
\hline$(121)$ & $s_{1} s_{2} s_{1}$ & $\left(-\lambda_{2}-2,-\lambda_{1}-2, \lambda_{1}+\lambda_{2}+\lambda_{3}+2\right)$ \\
\hline$(213)$ & $s_{2} s_{1} s_{3}$ & $\left(\lambda_{2}+\lambda_{3}+1,-\lambda_{1}-\lambda_{2}-\lambda_{3}-4, \lambda_{1}+\lambda_{2}+1\right)$ \\
\hline$(132)$ & $s_{1} s_{3} s_{2}$ & $\left(-\lambda_{1}-\lambda_{2}-3, \lambda_{1}+\lambda_{2}+\lambda_{3}+2,-\lambda_{2}-\lambda_{3}-3\right)$ \\
\hline$(321)$ & $s_{3} s_{2} s_{1}$ & $\left(\lambda_{2}, \lambda_{3},-\lambda_{1}-\lambda_{2}-\lambda_{3}-4\right)$ \\
\hline$(232)$ & $s_{2} s_{3} s_{2}$ & $\left(\lambda_{1}+\lambda_{2}+\lambda_{3}+2,-\lambda_{3}-2,-\lambda_{2}-2\right)$ \\
\hline$(1213)$ & $s_{1} s_{2} s_{1} s_{3}$ & $\left(-\lambda_{2}-\lambda_{3}-3,-\lambda_{1}-2, \lambda_{1}+\lambda_{2}+1\right)$ \\
\hline$(1232)$ & $s_{1} s_{2} s_{3} s_{2}$ & $\left(-\lambda_{1}-\lambda_{2}-\lambda_{3}-4, \lambda_{1}+\lambda_{2}+1,-\lambda_{2}-2\right)$ \\
\hline$(2132)$ & $s_{2} s_{1} s_{3} s_{2}$ & $\left(\lambda_{3},-\lambda_{1}-\lambda_{2}-\lambda_{3}-4, \lambda_{1}\right)$ \\
\hline$(1321)$ & $s_{1} s_{3} s_{2} s_{1}$ & $\left(-\lambda_{2}-2, \lambda_{2}+\lambda_{3}+1,-\lambda_{1}-\lambda_{2}-\lambda_{3}-4\right)$ \\
\hline$(2321)$ & $s_{2} s_{3} s_{2} s_{1}$ & $\left(\lambda_{2}+\lambda_{3}+1,-\lambda_{3}-2,-\lambda_{1}-\lambda_{2}-3\right)$ \\
\hline$(12132)$ & $s_{1} s_{2} s_{1} s_{3} s_{2}$ & $\left(-\lambda_{3}-2,-\lambda_{1}-\lambda_{2}-3, \lambda_{1}\right)$ \\
\hline$(12321)$ & $s_{1} s_{2} s_{3} s_{2} s_{1}$ & $\left(-\lambda_{2}-\lambda_{3}-3, \lambda_{2},-\lambda_{1}-\lambda_{2}-3\right)$ \\
\hline$(21321)$ & $s_{2} s_{1} s_{3} s_{2} s_{1}$ & $\left(\lambda_{3},-\lambda_{2}-\lambda_{3}-3,-\lambda_{1}-2\right)$ \\
\hline$(121321)$ & $s_{1} s_{2} s_{1} s_{3} s_{2} s_{1}$ & $\left(-\lambda_{3}-2,-\lambda_{2}-2,-\lambda_{1}-2\right)$ \\
\hline
\end{tabular}

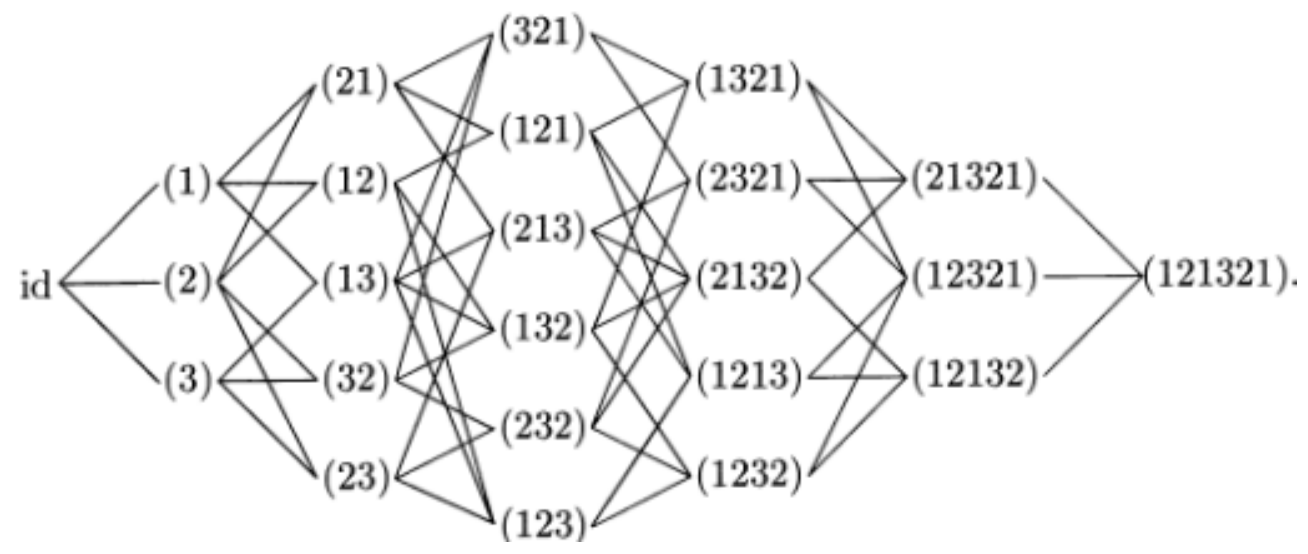

Figura A.2: Ordem de Bruhat $S_{4}$. 


\begin{tabular}{|c|c|c|}
\hline & $w$ & $w \pi$ \\
\hline id & id & $\left\{\alpha_{1}, \alpha_{2}, \alpha_{3}\right\}$ \\
\hline$(2134)$ & $s_{1}$ & $\left\{-\alpha_{1}, \alpha_{1}+\alpha_{2}, \alpha_{3}\right\}$ \\
\hline$(1324)$ & $s_{2}$ & $\left\{\alpha_{1}+\alpha_{2},-\alpha_{2}, \alpha_{2}+\alpha_{3}\right\}$ \\
\hline$(1243)$ & $s_{3}$ & $\left\{\alpha_{1}, \alpha_{2}+\alpha_{3},-\alpha_{3}\right\}$ \\
\hline$(3124)$ & $s_{2} s_{1}$ & $\left\{-\alpha_{1}-\alpha_{2}, \alpha_{1}, \alpha_{2}+\alpha_{3}\right\}$ \\
\hline$(2314)$ & $s_{1} s_{2}$ & $\left\{\alpha_{2},-\alpha_{1}-\alpha_{2}, \alpha_{1}+\alpha_{2}+\alpha_{3}\right\}$ \\
\hline$(2143)$ & $s_{1} s_{3}$ & $\left\{-\alpha_{1}, \alpha_{1}+\alpha_{2}+\alpha_{3},-\alpha_{3}\right\}$ \\
\hline$(1423)$ & $s_{3} s_{2}$ & $\left\{\alpha_{1}+\alpha_{2}+\alpha_{3},-\alpha_{2}-\alpha_{3}, \alpha_{2}\right\}$ \\
\hline$(1342)$ & $s_{2} s_{3}$ & $\left\{\alpha_{1}+\alpha_{2}, \alpha_{3},-\alpha_{2}-\alpha_{3}\right\}$ \\
\hline$(4123)$ & $s_{3} s_{2} s_{1}$ & $\left\{-\alpha_{1}-\alpha_{2}-\alpha_{3}, \alpha_{1}, \alpha_{2}\right\}$ \\
\hline$(3214)$ & $s_{1} s_{2} s_{1}$ & $\left\{-\alpha_{2},-\alpha_{1}, \alpha_{1}+\alpha_{2}+\alpha_{3}\right\}$ \\
\hline$(2413)$ & $s_{1} s_{3} s_{2}$ & $\left\{\alpha_{2}+\alpha_{3},-\alpha_{1}-\alpha_{2}-\alpha_{3}, \alpha_{1}+\alpha_{2}\right\}$ \\
\hline$(3142)$ & $s_{2} s_{1} s_{3}$ & $\left\{-\alpha_{1}-\alpha_{2}, \alpha_{1}+\alpha_{2}+\alpha_{3},-\alpha_{2}-\alpha_{3}\right\}$ \\
\hline$(2341)$ & $s_{1} s_{2} s_{3}$ & $\left\{\alpha_{2}, \alpha_{3},-\alpha_{1}-\alpha_{2}-\alpha_{3}\right\}$ \\
\hline$(1432)$ & $s_{2} s_{3} s_{2}$ & $\left\{\alpha_{1}+\alpha_{2}+\alpha_{3},-\alpha_{3},-\alpha_{2}\right\}$ \\
\hline$(4213)$ & $s_{1} s_{3} s_{2} s_{1}$ & $\left\{-\alpha_{2}-\alpha_{3},-\alpha_{1}, \alpha_{1}+\alpha_{2}\right\}$ \\
\hline$(4132)$ & $s_{2} s_{3} s_{2} s_{1}$ & $\left\{-\alpha_{1}-\alpha_{2}-\alpha_{3}, \alpha_{1}+\alpha_{2},-\alpha_{2}\right\}$ \\
\hline$(3412)$ & $s_{2} s_{1} s_{3} s_{2}$ & $\left\{\alpha_{3},-\alpha_{1}-\alpha_{2}-\alpha_{3}, \alpha_{1}\right\}$ \\
\hline$(3241)$ & $s_{1} s_{2} s_{1} s_{3}$ & $\left\{-\alpha_{2}, \alpha_{2}+\alpha_{3},-\alpha_{1}-\alpha_{2}-\alpha_{3}\right\}$ \\
\hline$(2431)$ & $s_{1} s_{2} s_{1} s_{3}$ & $\left\{\alpha_{2}+\alpha_{3},-\alpha_{3},-\alpha_{1}-\alpha_{2}\right\}$ \\
\hline$(4312)$ & $s_{2} s_{1} s_{3} s_{2} s_{1}$ & $\left\{-\alpha_{3},-\alpha_{1}-\alpha_{2}, \alpha_{1}\right\}$ \\
\hline$(4231)$ & $s_{1} s_{2} s_{3} s_{2} s_{1}$ & $\left\{-\alpha_{2}-\alpha_{3}, \alpha_{2},-\alpha_{1}-\alpha_{2}\right\}$ \\
\hline$(3421)$ & $s_{1} s_{2} s_{1} s_{3} s_{2}$ & $\left\{\alpha_{3},-\alpha_{2}-\alpha_{3},-\alpha_{1}\right\}$ \\
\hline$(4321)$ & $s_{1} s_{2} s_{1} s_{3} s_{2} s_{1}$ & $\left\{-\alpha_{3},-\alpha_{2},-\alpha_{1}\right\}$ \\
\hline
\end{tabular}




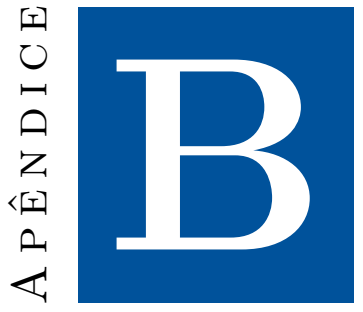

\section{Códigos dos reticulados de peso}

Os códigos em Wolfram Mathematica 12, que nos permitem construir os reticulados de peso de cada um dos $\mathfrak{s l}_{4}$-módulos simples apresentados neste trabalho são:

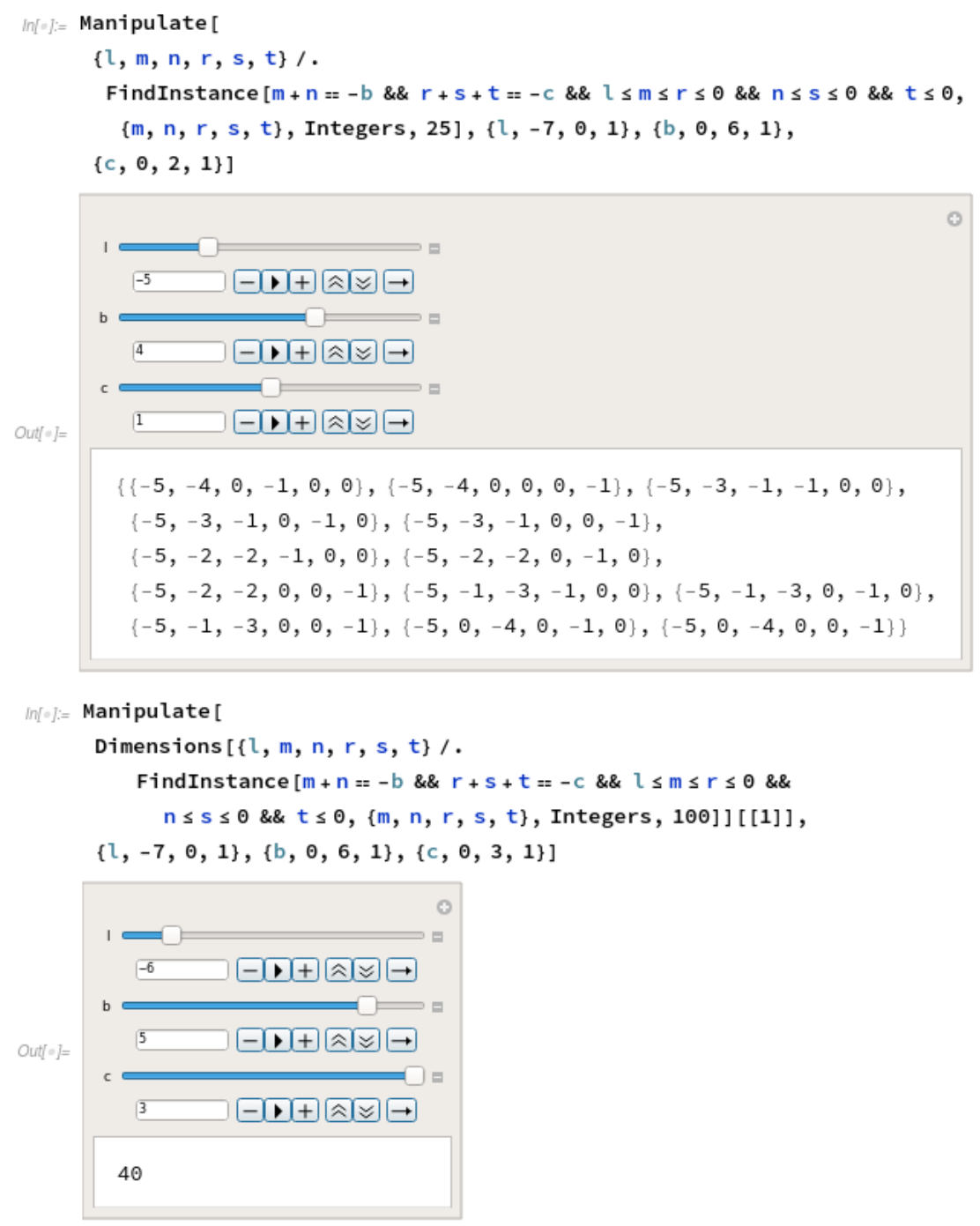

Figura B.1: Módulo de peso máximo genérico. 


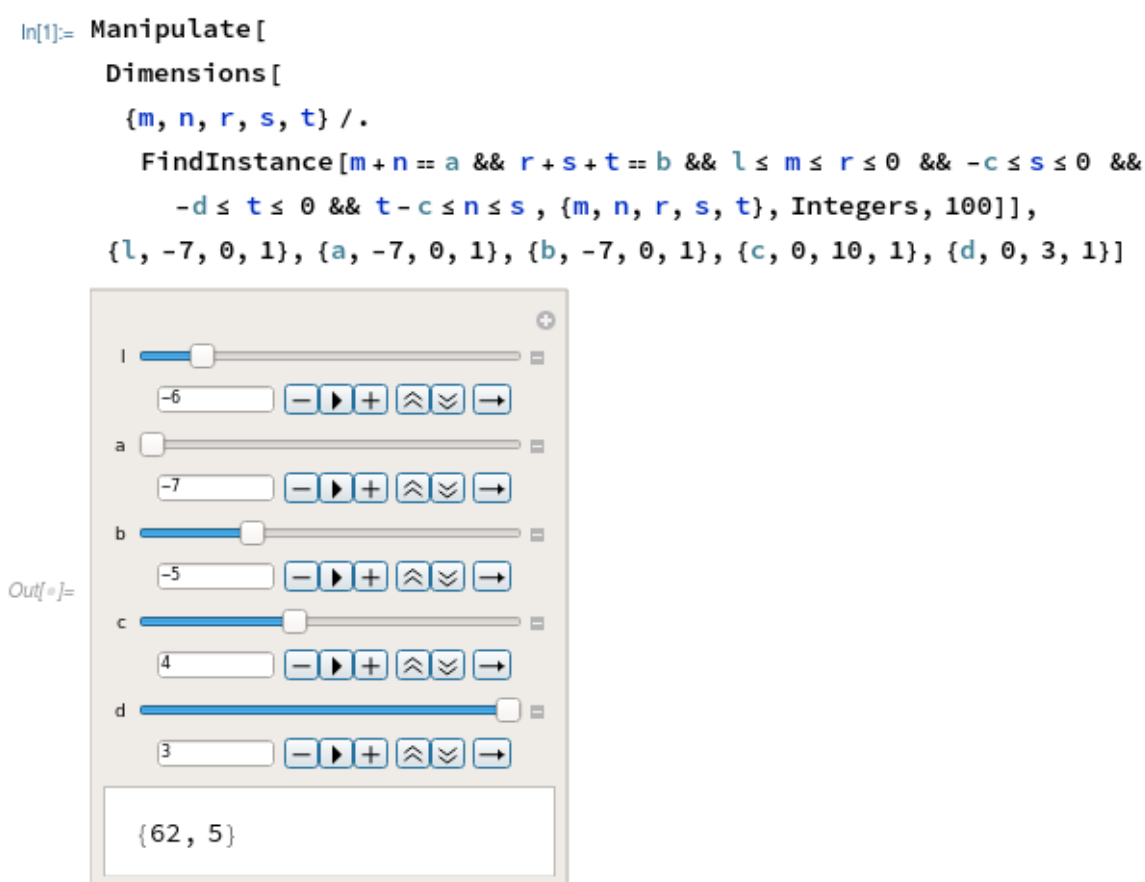

$\ln [2]:=$ Manipulate [

$\{m, n, r, s, t\} /$.

FindInstance $[m+n=a$ \&\& $r+s+t=b \quad \& \& \quad l \leq m \leq r \leq 0$ \&\& $-c \leq s \leq 0$ \&\&

$-d \leq t \leq 0 \& \& t-c \leq n \leq s,\{m, n, r, s, t\}$, Integers, 100], $\{l,-7,0,1\}$, $\{a,-7, \odot, 1\},\{b,-7, \odot, 1\},\{c, \odot, 10,1\},\{d, \odot, 3,1\}]$

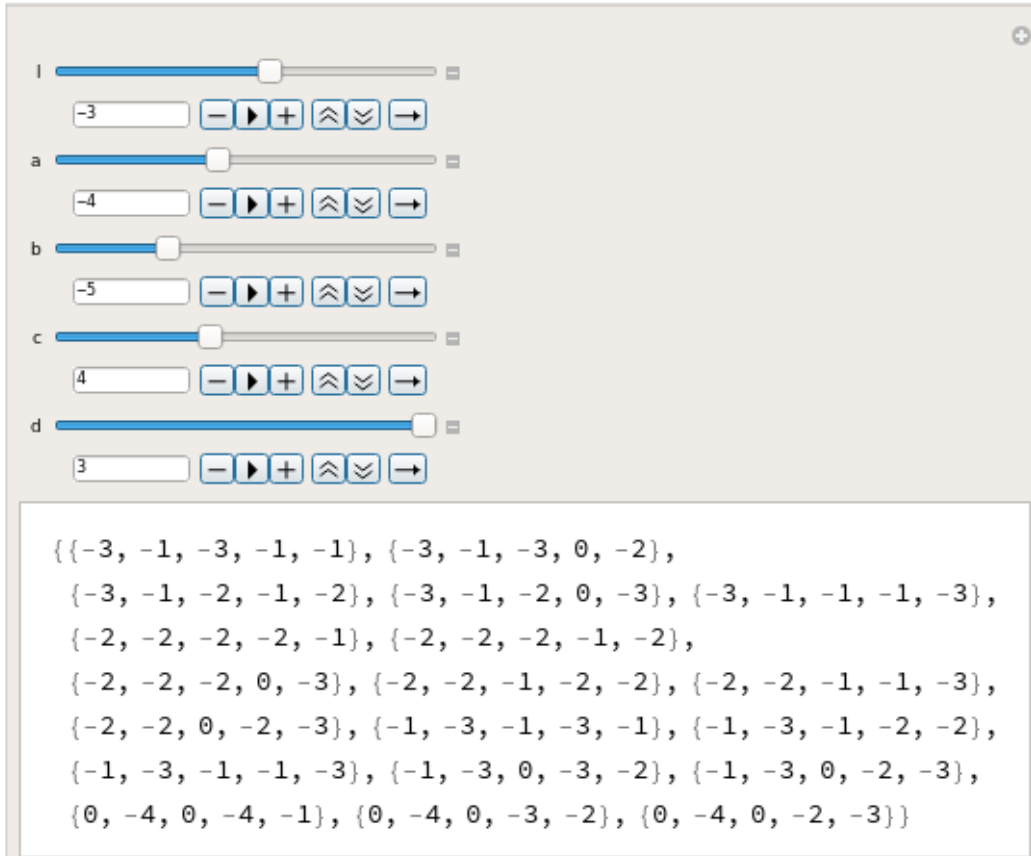

Figura B.2: Módulo de peso máximo limitado. 
$\ln [1]:=$ Manipulate [

Dimensions [

$\{m, n, r, s, t\} /$.

FindInstance $[m+n=a$ \&\& $r+s+t=b \quad \& \& m \leq r \leq 0 \quad$ \&\& $-c \leq s \leq 0 \quad$ \&\& $-d \leq t \leq 0 \& \& t-c \leq n \leq s,\{m, n, r, s, t\}$, Integers, 100]][[1]], $\{a,-7, \odot, 1\},\{b,-7, \odot, 1\},\{c, \odot, 10,1\},\{d, \odot, 3,1\}]$

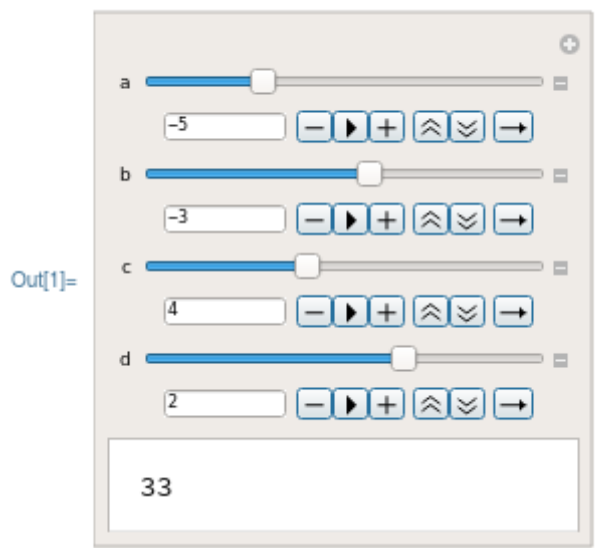

$\ln [-\sigma:=$ Manipulate [

$\{\mathrm{m}, \mathrm{n}, \mathrm{r}, \mathrm{s}, \mathrm{t}\} /$.

FindInstance $[m+n=a$ \&\& $r+s+t=b \quad \& \& m \leq r \leq 0 \quad$ \&\& $-c \leq s \leq 0$ \&\&

$-d \leq t \leq 0 \& \& t-c \leq n \leq s,\{m, n, r, s, t\}$, Integers, 100], $\{a,-7,0,1\}$,

$\{b,-7, \odot, 1\},\{c, 0,10,1\},\{d, \odot, 3,1\}]$

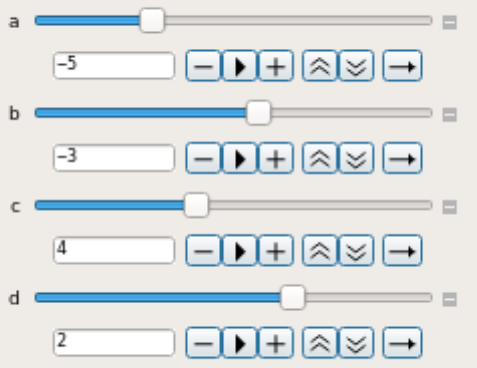

Out $[\cdot]=$

$\{\{-5, \odot,-3, \odot, \odot\},\{-5, \odot,-2, \odot,-1\},\{-5, \odot,-1, \odot,-2\}$,

$\{-4,-1,-3, \odot, \odot\},\{-4,-1,-2,-1, \odot\},\{-4,-1,-2, \odot,-1\}$,

$\{-4,-1,-1,-1,-1\},\{-4,-1,-1, \odot,-2\},\{-4,-1, \odot,-1,-2\}$,

$\{-3,-2,-3, \odot, \odot\},\{-3,-2,-2,-1, \odot\},\{-3,-2,-2, \odot,-1\}$,

$\{-3,-2,-1,-2, \odot\},\{-3,-2,-1,-1,-1\},\{-3,-2,-1, \odot,-2\}$,

$\{-3,-2, \odot,-2,-1\},\{-3,-2, \odot,-1,-2\},\{-2,-3,-2,-1,0\}$,

$\{-2,-3,-2, \odot,-1\},\{-2,-3,-1,-2, \odot\},\{-2,-3,-1,-1,-1\}$,

$\{-2,-3,-1, \odot,-2\},\{-2,-3, \odot,-3, \odot\},\{-2,-3, \odot,-2,-1\}$,

$\{-2,-3, \odot,-1,-2\},\{-1,-4,-1,-2,0\},\{-1,-4,-1,-1,-1\}$,

$\{-1,-4,-1, \odot,-2\},\{-1,-4, \odot,-3, \odot\},\{-1,-4, \odot,-2,-1\}$,

$\{-1,-4, \odot,-1,-2\},\{\odot,-5, \odot,-2,-1\},\{\odot,-5, \odot,-1,-2\}\}$

Figura B.3: Módulo induzido de $\mathfrak{s l}_{2}$. 

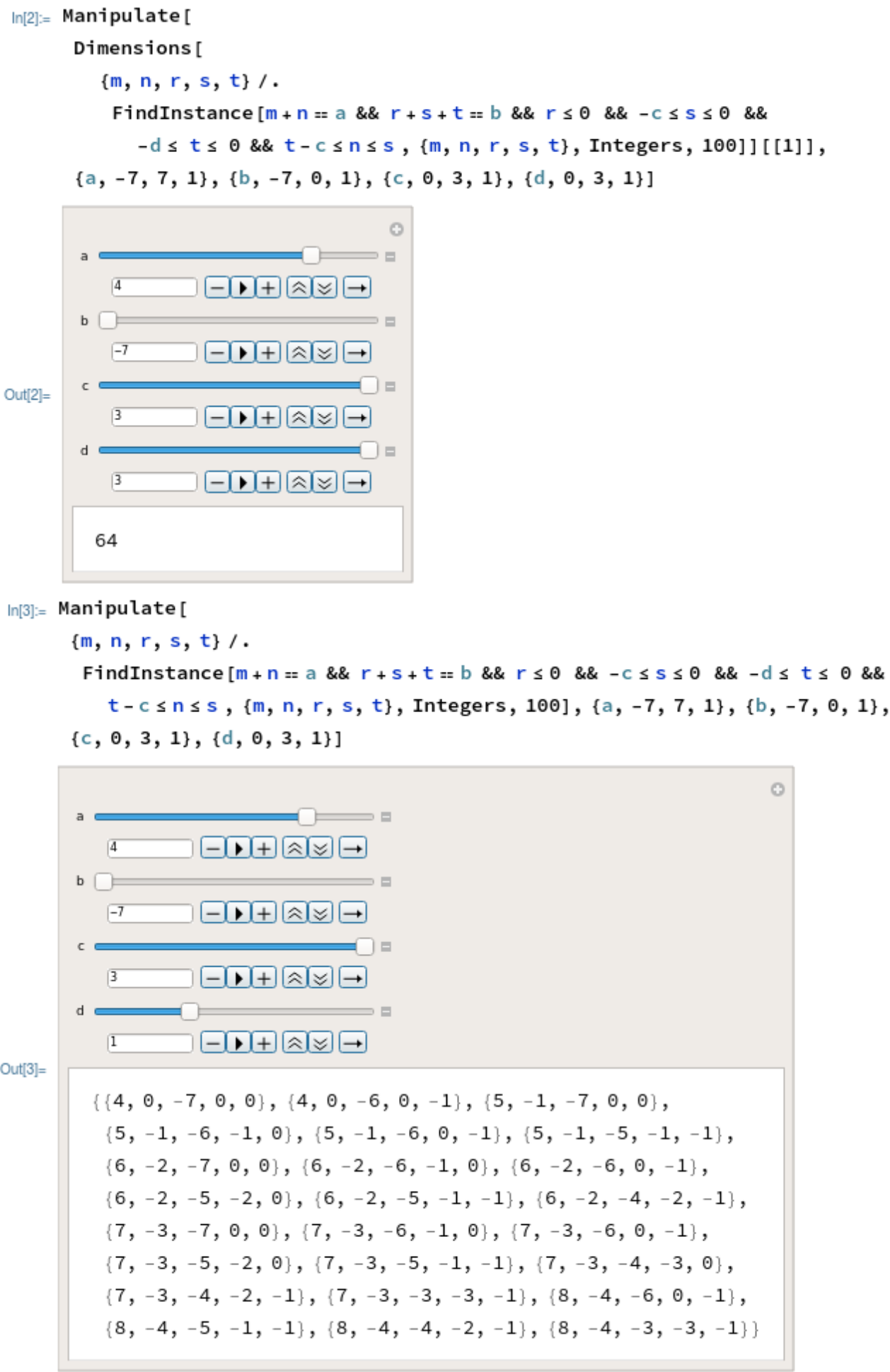

Figura B.4: Módulo induzido de $\mathfrak{s l}_{3}$. 
$\ln [4]:=$ Manipulate [

Dimensions $[\{m, n, r, s, t\} /$.

FindInstance $[m+n=a$ \&\& $r+s+t=b \quad \& \&-c \leq s \leq 0$ \&\&

$-d \leq t \leq 0 \& \& t-c \leq n \leq s,\{m, n, r, s, t\}$, Integers, 100]][[1]],

$\{\mathrm{a},-7,7,1\},\{\mathrm{b},-7,0,1\},\{\mathrm{c}, 0,3,1\},\{\mathrm{d}, 0,3,1\}]$

Out $[4]=$

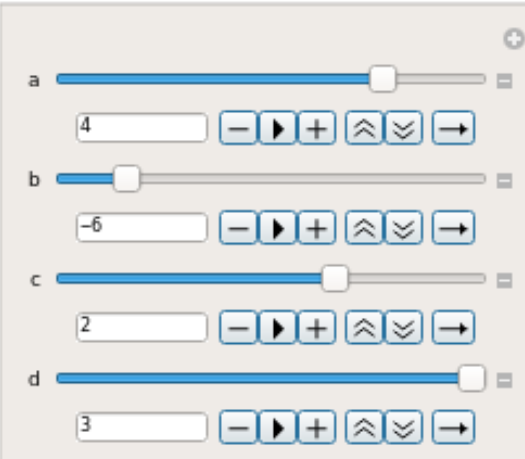

42

$\ln [5]:=$ Manipulate [

$\{\mathrm{m}, \mathrm{n}, \mathrm{r}, \mathrm{s}, \mathrm{t}\} /$.

FindInstance $[m+n=a \& \& \quad r+s+t=b \quad$ \&\& $-c \leq s \leq 0$ \&\&

$-d \leq t \leq 0$ \&\& $t-c \leq n \leq s,\{m, n, r, s, t\}$, Integers, 100],

$\{a,-7,7,1\},\{b,-7,0,1\},\{c, 0,3,1\},\{d, 0,3,1\}]$

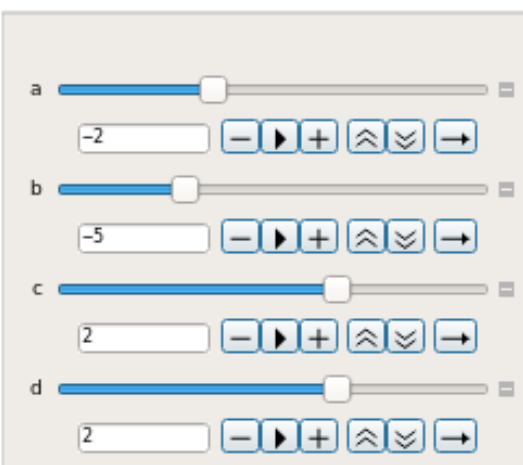

Out[5]=

$$
\begin{aligned}
& \{\{-2, \odot,-5, \odot, \odot\},\{-2, \odot,-4, \odot,-1\}, \\
& \{-2, \odot,-3, \odot,-2\},\{-1,-1,-5, \odot, \odot\},\{-1,-1,-4,-1, \odot\}, \\
& \{-1,-1,-4, \odot,-1\},\{-1,-1,-3,-1,-1\}, \\
& \{-1,-1,-3, \odot,-2\},\{-1,-1,-2,-1,-2\}, \\
& \{\odot,-2,-5, \odot, \odot\},\{\odot,-2,-4,-1, \odot\},\{\odot,-2,-4, \odot,-1\}, \\
& \{\odot,-2,-3,-2, \odot\},\{\odot,-2,-3,-1,-1\},\{\odot,-2,-3, \odot,-2\}, \\
& \{\odot,-2,-2,-2,-1\},\{\odot,-2,-2,-1,-2\},\{\odot,-2,-1,-2,-2\}, \\
& \{1,-3,-4, \odot,-1\},\{1,-3,-3,-1,-1\},\{1,-3,-3, \odot,-2\}, \\
& \{1,-3,-2,-2,-1\},\{1,-3,-2,-1,-2\},\{1,-3,-1,-2,-2\}, \\
& \{2,-4,-3, \odot,-2\},\{2,-4,-2,-1,-2\},\{2,-4,-1,-2,-2\}\},
\end{aligned}
$$

Figura B.5: $\mathfrak{s l}_{4}$-módulo cuspidal. 


\section{Referências Bibliográficas}

[ABD04] T. Abe, G. Buhl and C. Dong. Rationality, regularity, and $C_{2}$-cofiniteness. Trans. Amer. Math. Soc., 356(8):3391-3402, 2004. 26

[Ada16] D. Adamović. A realization of certain modules for the $N=4$ superconformal algebra and the affine Lie algebra $A_{2}^{(1)}$. Transform. Groups, 21(2):299-327, 2016. xi

[AFR17] T. Arakawa, V. Futorny and L. E. Ramirez. Weight representations of admissible affine vertex algebras. Comm. Math. Phys., 353(3):1151-1178, 2017. v, vii, xii, xiv, xvi, xvii, xviii, 32, 34, 36, 39, 61, 100, 102, 104, 105, 113, 114, 117

[AL03] H. H. Andersen and N. Lauritzen. Twisted verma modules. Basel: Birkhäuser, 1-26, 2003. xv

[AM95] D. Adamović and A. Milas. Vertex operator algebras associated to modular invariant representations for $A_{1}^{(1)}$. Math. Res. Lett., 2:563-575, 1995. xi

[Ara15a] T. Arakawa. Associated varieties of modules over Kac-Moody algebras and C2Cofiniteness of W-Algebras. Int. Math. Res. Not. IMRN, 2015(22):11605-11666, 2015. 34,37

[Ara15b] T. Arakawa. Rationality of W-algebras: principal nilpotent cases. Ann. of Math. (2), 182(2):565-694, 2015. xiii, xviii, 32, 35

[Ara16] T. Arakawa. Rationality of admissible affine vertex algebras in the category $\mathcal{O}$. Duke Math. J., 165(1):67-93, 2016. v, vii, 19, 30, 32

[Ara17] T. Arakawa. Introduction to $W$-Algebras and their representation theory. Springer International Publishing, Cham, 179-250, 2017. 3, 100

[Ark04] S. Arkhipov. Algebraic construction of contragradient quasi-verma modules in positive characteristic. Adv. Stud. Pure Math., 40:27-68, 2004. xv

[AS03] H. H. Andersen and C. Stroppel. Twisting functors on $\mathcal{O}$. Represent. Theory, 7:681-699, 2003. 7

[BB82] W. Borho and J.-L. Brylinski. Differential operators on homogeneous spaces. I: Irreducibility of the associated variety for annihilators of induced modules. Invent. Math., 69:437-476, 1982. 35

[BB05] A. Björner and F. Brenti. Combinatorics of Coxeter groups., volume 231. New York, NY: Springer, 2005. xix, 119

[Bor86] R. E. Borcherds. Vertex algebras, Kac-Moody algebras, and the Monster. Proc. Natl. Acad. Sci. USA, 83(10):3068-3071, 1986. 22, 28

[Car05] R. Carter. Lie algebras of finite and affine type., volume 96. Cambridge: Cambridge University Press, 2005. xix, 119 
[CF94] A. J. Coleman and V. Futorny. Stratified l-modules. J. Algebra, 163(1):219-234, 1994. 5

[CM93] D.H. Collingwood and W.M. McGovern. Nilpotent Orbits In Semisimple Lie Algebra: An Introduction. Mathematics series. Taylor \& Francis, 1993. 33, 37

[DFG09] I. Dimitrov, V. Futorny and D. Grantcharov. Parabolic sets of roots. Providence, RI: Amer. Math. Soc. (AMS), 61-73, 2009. 5

[DFO94] Y. Drozd, V. Futorny and S. Ovsienko. Harish-Chandra Subalgebras and Gelfand-Zetlin Modules. Math. Phys. Sci., 424:72-89, 1994. 42, 43

[Dix77] J. Dixmier. Enveloping algebras. Amsterdam : North-Holland Pub. Co. ; Amsterdam/New York/Oxford, 1977. Translation of Algèbres enveloppantes. xix, 1, 8, 9, 70, 119

[DLM97] C. Dong, H. Li and G. Mason. Regularity of rational vertex operator algebras. Adv. Math., 132(1):148-166, 1997. 25, 26

[DLM98] C. Dong, H. Li and G. Mason. Vertex operator algebras and associative algebras. J. Algebra, 206(1):67-96, 1998. xi, 29

[DMP00] I. Dimitrov, O. Mathieu and I. Penkov. On the structure of weight modules. Trans. Amer. Math. Soc., 352(6):2857-2869, 2000. 15

[DSK06] A. De Sole and V. Kac. Finite vs affine W-algebras. Jpn. J. Math., 1(1):137-261, 2006. $21,22,26$

[Duf77] M. Duflo. Sur la Classification des Ideaux Primitifs Dans L'algebre Enveloppante d'une Algebre de Lie Semi-Simple. Ann. of Math. (2), 105(1):107-120, 1977. 9, 32

[FB04] E. Frenkel and D. Ben-Zvi. Vertex algebras and algebraic curves. 2nd revised and expanded ed., volume 88. Providence, RI: Amer. Math. Soc. (AMS), 2nd revised and expanded ed., 2004. 19, 21, 24, 26

[Fer90] S. L. Fernando. Lie algebra modules with finite dimensional weight spaces. I. Trans. Amer. Math. Soc., 322(2):757-781, 1990. xvii, 5, 10, 12, 73

[FGR15] V. Futorny, D. Grantcharov and L. E. Ramirez. Irreducible generic Gelfand-Tsetlin modules of $\mathfrak{g l}(n)$. SIGMA, 11(018):13, 2015. 55

[FGR16] V. Futorny, D. Grantcharov and L. E. Ramirez. Singular Gelfand-Tsetlin modules of $\mathfrak{g l}(n)$. Adv. Math., 290:453-482, 2016. 75

[FGR20] V. Futorny, D. Grantcharov and L. E. Ramirez. Classification of irreducible GelfandTsetlin modules of $\mathfrak{s t}(3)$. arXiv:1812.0713\%v2[math.RT], 2020. 58, 67, 87, 92

[FGRZ20] V. Futorny, D. Grantcharov, L. E. Ramirez and P. Zadunaisky. Bounds of GelfandTsetlin multiplicities and tableaux realizations of Verma modules. J. Algebra, 556:412 436, 2020. 61, 63

[FHK21] V. Futorny, O. A. Hernández Morales and L. Křižka. Admissible representations of simple affine vertex algebras. In progress, 2021. xiii, xviii

[FHL93] I. B. Frenkel, Y. Z. Huang and J. Lepowsky. On axiomatic approaches to vertex operator algebras and modules. Mem. Amer. Math. Soc., 494:64, 1993. xviii, 21, 24

[FHR20] V. Futorny, O. A. Hernández Morales and L. E. Ramirez. Simple modules for Affine vertex algebras in the minimal nilpotent orbit. arXiv:2002.05568[math.RT], 2020. xiii, xviii, 10, 16, 17, 32, 58, 61, 63, 64, 65, 66, 67, 69, 70, 74, 75, 83, 102, 103, 104, 105 
[FK18] V. Futorny and I. Kashuba. Structure of parabolically induced modules for affine Kac-Moody algebras. J. Algebra, 500:362-374, 2018. 5, 20

[FK19a] V. Futorny and L. Křižka. Geometric construction of Gelfand-Tsetlin modules over simple lie algebras. J. Algebra Appl, 223(11):4901 - 4924, 2019. xv, xviii, 6, 7

[FK19b] V. Futorny and L. Křižka. Twisting functors and Gelfand-Tsetlin modules over semisimple lie algebras. arXiv:1902.02269v1[math.RT], 2019. xv, 6, 7

[FK20] V. Futorny and L. Křžzka. Positive energy representations of affine vertex algebras. Comm. Math. Phys., 2020. xii, 7, 8, 33, 36

[FKW92] E. Frenkel, V. Kac and M. Wakimoto. Characters and fusion rules for W-algebras via quantized Drinfeld-Sokolov reduction. Comm. Math. Phys., 147(2):295-328, 1992. xiv

[FRZ19] V. Futorny, L. E. Ramirez and J. Zhang. Combinatorial construction of Gelfand-Tsetlin modules for $\mathfrak{g l}_{n}$. Adv. Math., 343:681-711, 2019. v, vii, xiii, xiv, xviii, 41, 42, 43, 45, $49,50,54,61$

[FST98] B. L. Feigin, A. M. Semikhatov and I. Yu. Tipunin. Equivalence between chain categories of representations of affine $\mathrm{sl}(2)$ and $\mathrm{N}=2$ superconformal algebras. J. Math. Phys., 39(7):3865-3905, 1998. xi

[Fut97] V. Futorny. Representations of affine Lie algebras, Queen's Papers in Pure and Appied Mathematics. Queen's University, Kingston, 106, 1997. 5, 20

[FZ92] I. B. Frenkel and Y. Zhu. Vertex operator algebras associated to representations of affine and Virasoro algebras. Duke Math. J., 66(1):123-168, 1992. xi, xii, xviii, 23, 27, $28,30,100$

[GP20] D. Grantcharov and I. Penkov. SIMPLE BOUNDED WEIGHT MODULES OF $\mathfrak{s l}(\infty)$, $\mathfrak{o}(\infty), \mathfrak{s p}(\infty)$. Transform. Groups, 25:1125-1160, 2020. 16, 17

[Gra14] D. Grantcharov. Twisted Localization of Weight Modules. Springer International Publishing, 185-206, 2014. 15

[GT50] I.M. Gelfand and M.L. Tsetlin. Finite-dimensional representations of the group of unimodular matrices. Dokl. Akad. Nauk USSR, 71:825-828, 1950. 43

[GT88] I. Gelfand and M. Tsetlin. Finite-dimensional representations of the group of unimodular matrices. Springer-Verlag Berlin Heidelberg, 653-656, 1988. (The article was originally published in 1950, in Russian.). 45

[He17] X. He. Higher level Zhu algebras are subquotients of universal enveloping algebras. $J$. Algebra, 491:265 - 279, 2017. xi

[Hum80] J. E. Humphreys. Introduction to Lie algebras and representation theory. 3rd printing, rev., volume 9. Springer, New York, NY, 1980. xix, 1, 119

[Hum08] J. E. Humphreys. Representations of semisimple Lie algebras in the BGG category $\mathcal{O}$., volume 94. Providence, RI: Amer. Math. Soc. (AMS), 2008. xv, 3, 7, 8, 9, 62

[Jan77] J. C. Jantzen. Kontravariante Formen auf induzierten Darstellungen halbeinfacher LieAlgebren. Math. Ann., 226:53-65, 1977. xiv

[Jos79] A. Joseph. Dixmier's Problem for Verma and Principal Series Submodules. J. Lond. Math. Soc. (2), s2-20(2):193-204, 1979. 10 
[Jos85] A. Joseph. On the associated variety of a primitive ideal. J. Algebra, 93(2):509-523, 1985. 34

[Jos98] A. Joseph. Sur l'annulateur d'un module de Verma. Springer Netherlands, Dordrecht, 237-300, 1998. 2, 9

[Kac90] V. Kac. Infinite-Dimensional Lie Algebras. Cambridge University Press, 3 edição, 1990. xviii, 19, 21

[Kac98] V. Kac. Vertex algebras for beginners. 2nd ed., volume 10. Providence, RI: Amer. Math. Soc., 1998. xviii, 19, 21, 24, 30

[Kac17] V. Kac. Introduction to Vertex Algebras, Poisson Vertex Algebras, and Integrable Hamiltonian PDE. Springer International Publishing, Cham, 3-72, 2017. 19, 21, 25, 30

[KR19a] K. Kawasetsu and D. Ridout. Relaxed Highest-Weight Modules I: Rank 1 Cases. Comm. Math. Phys., 368(2):627-663, 2019. xii

[KR19b] K. Kawasetsu and D. Ridout. Relaxed highest-weight modules II: classifications for affine vertex algebras. arXiv:1906.02935v2[math.RT], 2019. xii

[KW88] V. Kac and M. Wakimoto. Modular invariant representations of infinite-dimensional lie algebras and superalgebras. Proc. Natl. Acad. Sci. USA, 85(14):4956-4960, 1988. 11

[KW89] V. Kac and M. Wakimoto. Classification of modular invariant representations of affine algebras., volume 7. Adv. Ser. Math. Phys., 138-177, 1989. xi, xviii, 32, 38

[KW08] V. Kac and M. Wakimoto. On Rationality of W-algebras. Transform. Groups, 13(3):671713, 2008. xviii, 31, 32, 36

[Li99] H. Li. Some finiteness properties of regular vertex operator algebras. J. Algebra, 212(2):495-514, 1999. 26

[LL04] J. Lepowsky and H. Li. Introduction to vertex operator algebras and their representations., volume 227. Boston, MA: Birkhäuser, 2004. 21, 22, 24, 25

[Mar10] L. A. B. San Martin. Álgebras de Lie, volume 2a. edição. Editora da Unicamp, 2010. 1,119

[Mat00] O. Mathieu. Classification of irreducible weight modules. Ann. Inst. Fourier, 50(2):537592, 2000. xiii, xv, xvii, 5, 10, 11, 12, 13, 14, 15, 16, 58, 87

[Maz98] V. Mazorchuk. Tableaux realization of generalized Verma modules. Can. J. Math., $50(4): 816-828,1998$. xiv, 61

[Maz03] V. Mazorchuk. Quantum deformation and tableaux realization of simple dense $\mathfrak{g l}(n, \mathbb{C})$ modules. J. Algebra Appl., 2(1):1-20, 2003. v, vii, xviii, 73, 87

[Maz10] V. Mazorchuk. Lectures on $\mathfrak{s l}_{2}(\mathbb{C})$-modules. Imperial College Press, 2010. xii, 11, 70

[MRS01] J.C. McConnell, J.C. Robson and L.W. Small. Noncommutative Noetherian Rings. Graduate studies in mathematics. Amer. Math. Soc., 2001. 6

[oGVAG04] University of Georgia VIGRE Algebra Group. Varieties of nilpotent elements for simple Lie algebras I: Good primes. J. Algebra, 280(2):719 - 737, 2004. 37

[SW20] T. Silverthorne and B. Webster. Gelfand-Tsetlin modules: canonicity and calculations. arXiv:2011.06029[math.RT], 2020. 63 
[Wan99] W. Wang. Dimension of a minimal nilpotent orbit. Proc. Amer. Math. Soc, 127(3):935936, 1999. 100

[Zel73] D. P. Zelobenko. Compact Lie groups and their representations, volume 40. Amer. Math. Soc. (AMS), Providence, RI, 1973. Translated from the Russian by Israel Program for Scientific Translations. 46

[Zhu96] Y. Zhu. Modular invariance of characters of vertex operator algebras. J. Amer. Math. Soc., 9(1):237-302, 1996. xi, xii, xviii, 25, 26, 27, 28 